\title{
Strukturbildung und Rauigkeiten an Grenzflächen des Ni-Ag-Legierungssystems
}

\section{Dissertation}

\author{
zur Erlangung des Doktorgrades \\ der Mathematisch-Naturwissenschaftlichen Fakultäten \\ der Georg-August-Universität zu Göttingen
}

vorgelegt von

\section{Jan Petersen}

aus Eckernförde

Göttingen 2008 
Referent

Korreferent
Prof. Dr. S. G. Mayr

Prof. Dr. R. Kirchheim

Tag der mündlichen Prüfung: $\quad$ 21.04.2008 


\section{Inhaltsverzeichnis}

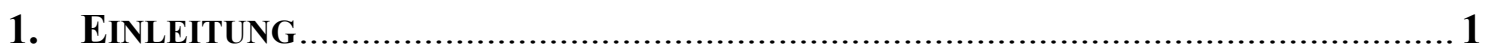

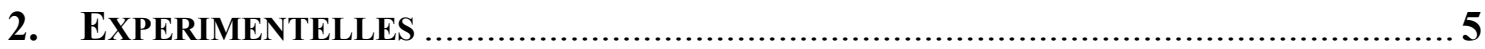

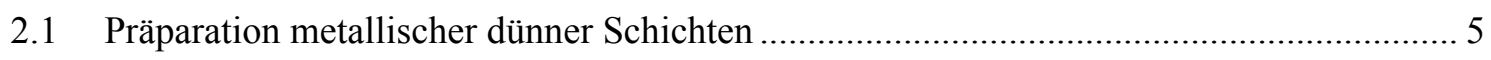

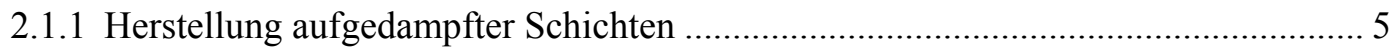

2.1.1.1 Die Ultrahochvakuumanlage .................................................................. 5

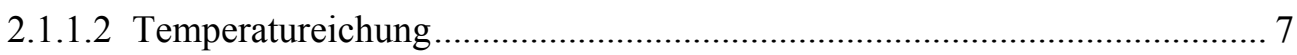

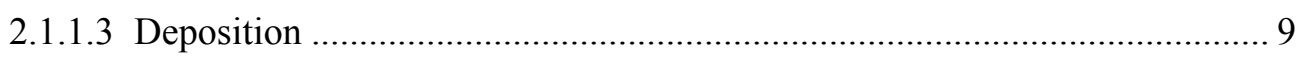

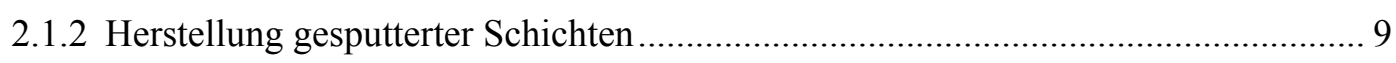

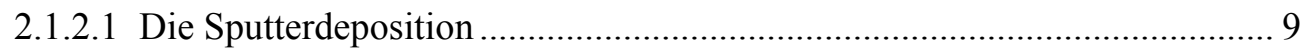

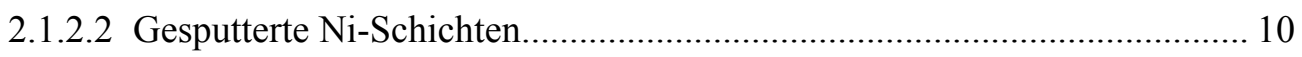

2.1.2.3 Gesputterte NiAg-Schichten............................................................ 10

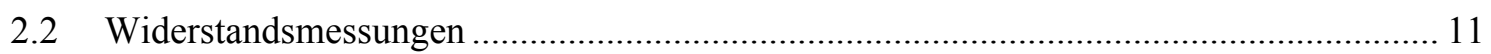

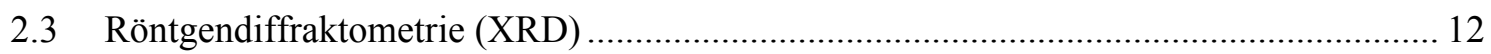

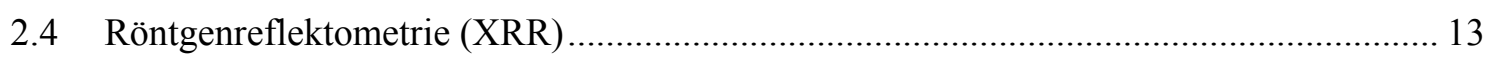

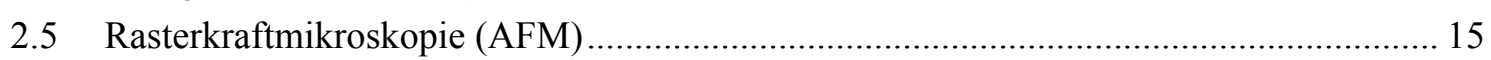

2.5.1 Statistische Analyse mittels der Autokorrelationsfunktion (ACF) ......................... 16

2.5.2 Berechnung der spektralen Leistungsdichte (PSD) ............................................. 17

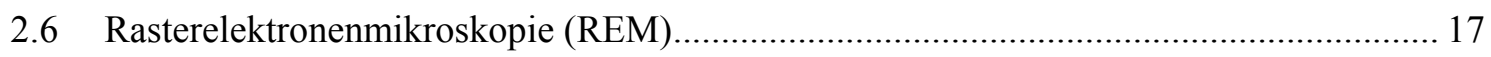

2.7 Energiedispersive Röntgenspektroskopie (EDX) ............................................................. 18

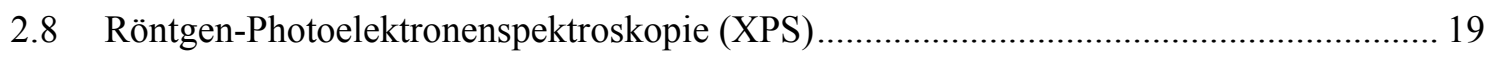

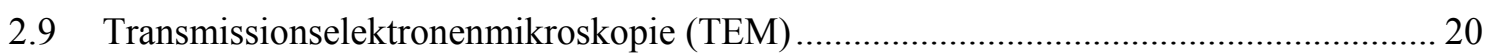

2.10 Magnetisierungsmessungen mittels magnetooptischen Kerreffekts (MOKE) ........ 22

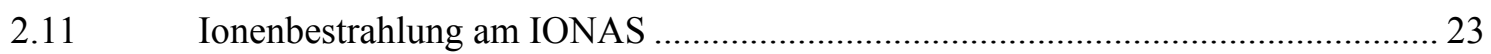

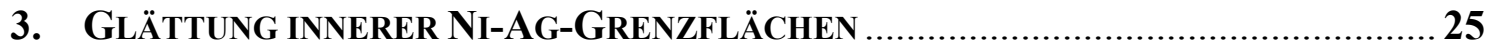

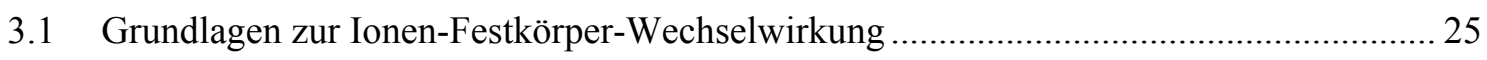

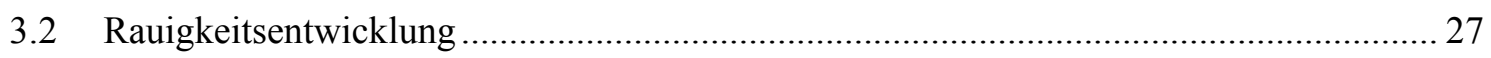

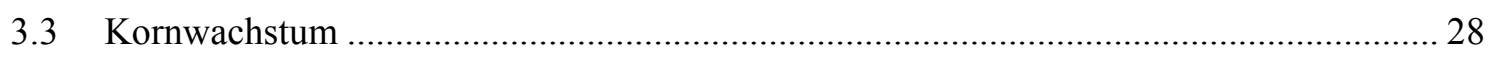

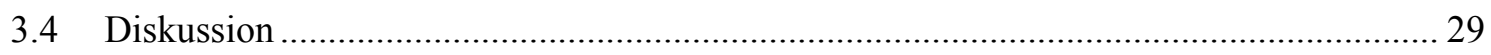

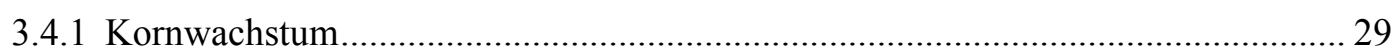

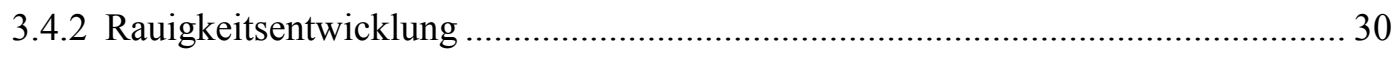




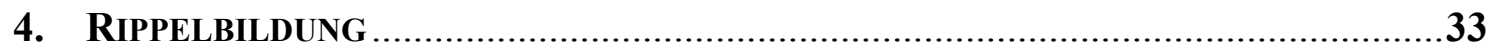

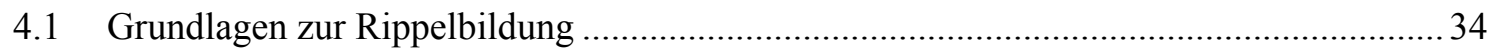

4.2 Herstellung und Charakterisierung rippelstrukturierter Substrate ........................................ 38

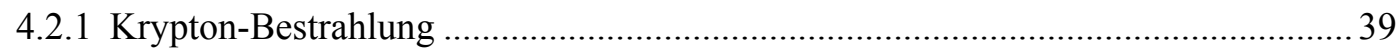

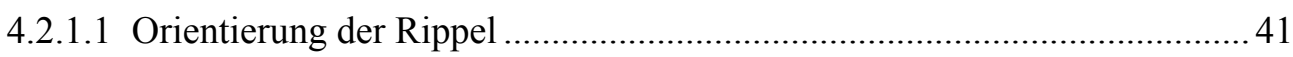

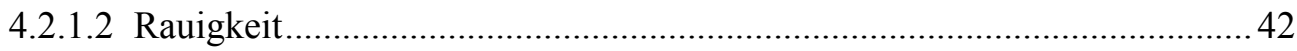

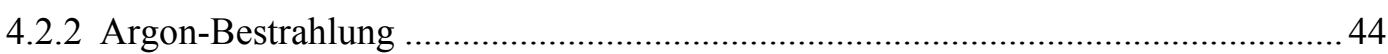

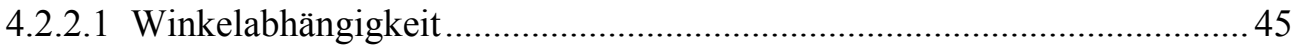

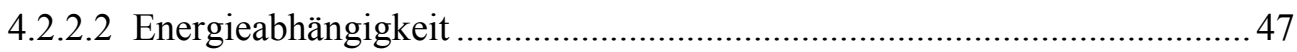

4.2.2.3 Hochgradig geordnete Wellenstrukturen ................................................ 50

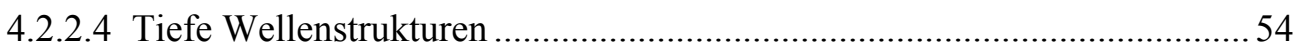

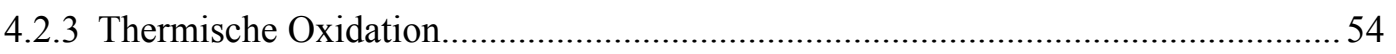

5. SELBSTORGANISATION WÄHREND DER DEPOSITION ...........................................57

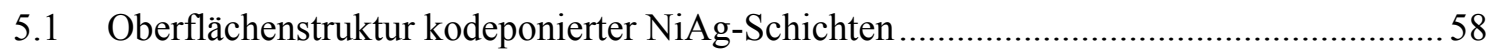

5.1.1 Einfluss der kurzwelligen, geordneten Rippelstruktur......................................... 58

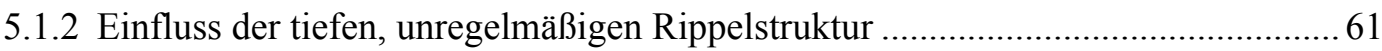

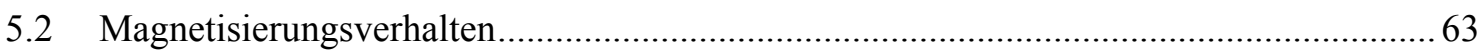

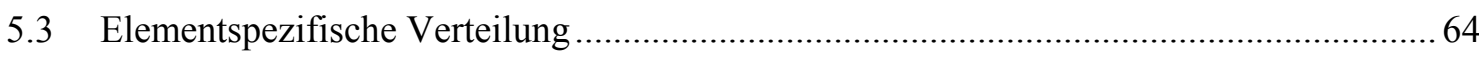

5.4 Strukturentwicklung in Ni-Ag-Schichten während thermischer Behandlung .................... 67

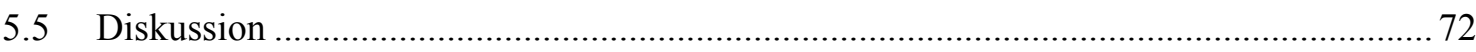

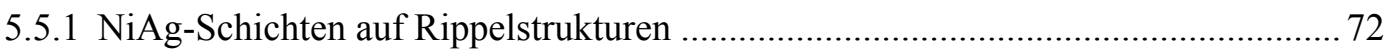

5.5.2 Thermische Behandlung von Ni-Ag-Schichten ...................................................... 76

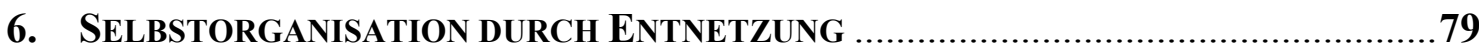

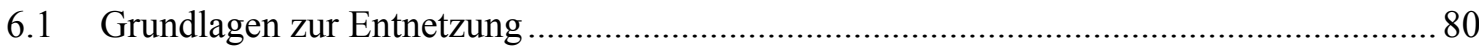

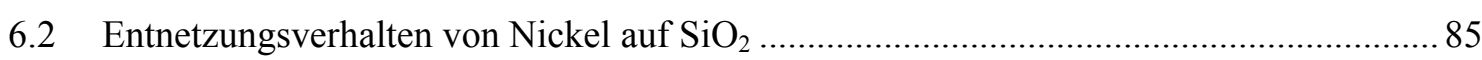

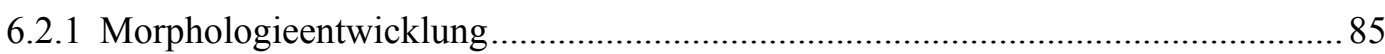

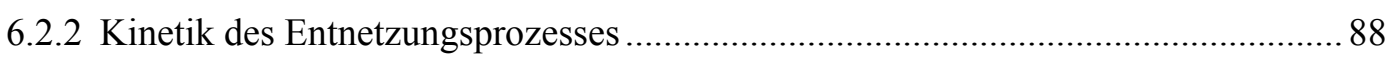

6.2.2.1 Widerstandsverlauf während der Deposition.......................................... 88

6.2.2.2 Widerstandsverlauf während der Entnetzung ........................................... 89

6.2.2.3 Einfluss von Sauerstoff............................................................................ 91

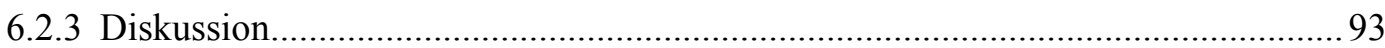

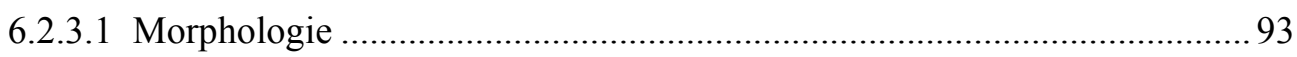

6.2.3.2 Widerstandsverhalten und Kinetik........................................................... 95

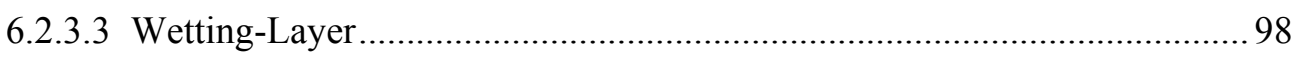

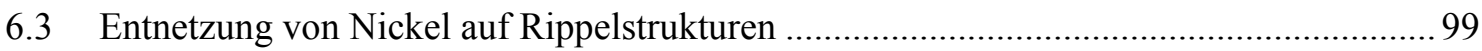

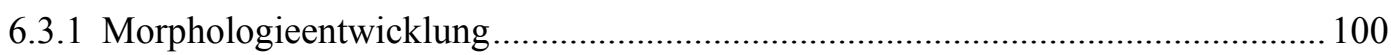

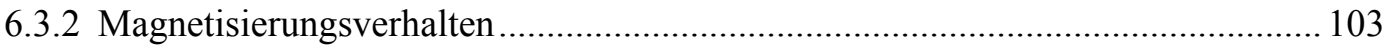

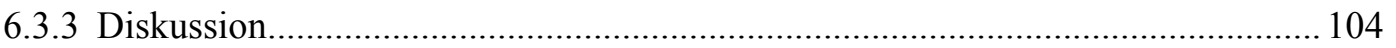




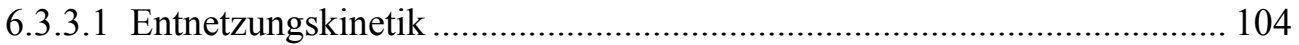

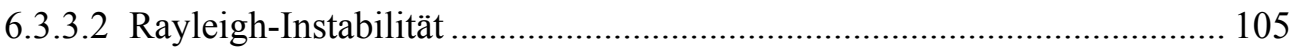

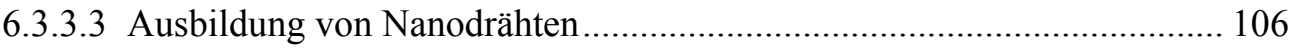

6.3.3.4 Magnetisierungsverhalten................................................................ 107

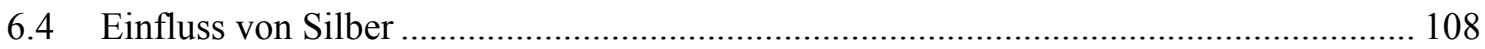

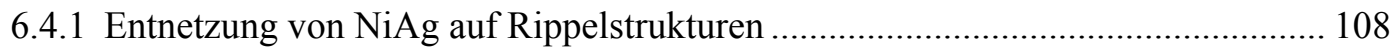

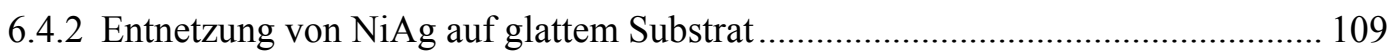

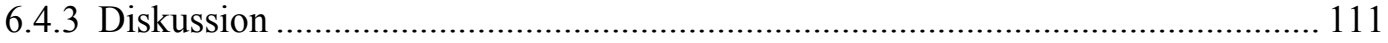

6.4.3.1 Morphologieentwicklung auf Rippelstrukturen...................................... 111

6.4.3.2 Entnetzungsmechanismus.............................................................. 112

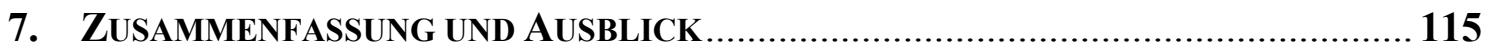

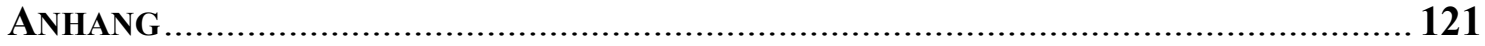

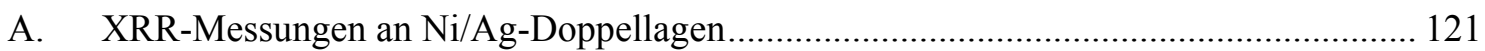

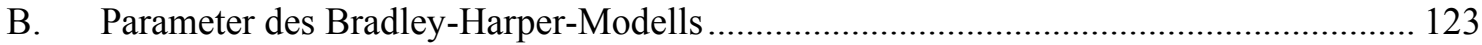

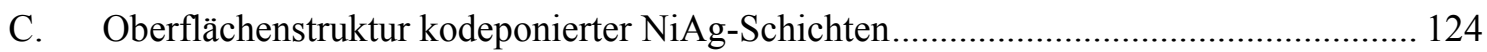

D. AFM-Aufnahmen der Entnetzung von Nickel auf glattem Substrat .............................. 126

E. AFM-Aufnahmen der Entnetzung von Nickel auf rippelstrukturiertem Substrat ........... 127

F. Temperaturabhängigkeit des Widerstandes einer 100nm dicken Nickelschicht ............. 128

G. Grenzflächenuntersuchung einer entnetzten Nickelschicht............................................ 129

H. Entnetzung einer 10nm Nickelschicht auf rippelstrukturiertem Substrat......................... 130

I. Entnetzung einer sputterdeponierten Nickelschicht ..................................................... 131

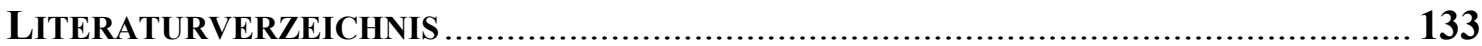

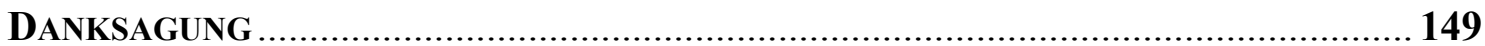

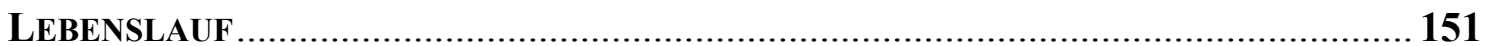





\section{Kapitel}

\section{Einleitung}

Viele physikalische Phänomene, die sich der Mensch auf technischer Ebene nutzbar zu machen bestrebt ist, basieren auf einer speziellen Anordnung von Materie und erfordern besondere Strukturierungsverfahren für eine gezielte Anwendung. Im Rahmen der Dünnschichttechnologie, in der neben mechanischen Schutzschichten auch optische, elektrische und magnetische Funktionsschichten zum Einsatz kommen, ist häufig die Morphologie der Grenzflächen von entscheidender Bedeutung. Der effiziente Einsatz von ultradünnen Zwischenschichten in Magnetowiderstandssystemen beispielsweise, deren Anwendungspotential unter anderem in magnetischen Speichern und Festplattenleseköpfen liegt, bedingt glatte Grenzflächen auf mesoskopischer Ebene, um ein definiertes Schaltverhalten und eine hohe thermische Stabilität zu erzielen sowie Kurzschlüsse infolge von Schichtdickenschwankungen zu vermeiden [Bin89, Moo95]. Die systematische Modifikation der Oberflächenstruktur ist vor allem im Bereich der magnetischen Speichermedien und der Nanotechnologie von Interesse, wo entweder glatte oder speziell strukturierte Oberflächen erforderlich sind.

Als Verfahren zur Präparation bis zu atomar glatter Oberflächen stellt die Ionenbestrahlung ein effektives Werkzeug dar. Für niederenergetische Ionenbestrahlung von wenigen keV besitzt der infolge resultierender Kinetik zur Aufrauung führende oberflächliche Sputterprozess entscheidenden Charakter [Bra88]. Im höherenergetischen Bereich, in dem weiterhin nuklearer Energieverlust überwiegt, gewinnen Effekte, welche mit der Ausbildung lokaler Kollisionskaskaden in das Target hinein einhergehen, zunehmend an Bedeutung. Die Kinetik wird hierbei von ballistischen Vorgängen und Defekterzeugung sowie lokalem Aufschmelzen innerhalb sogenannter Thermal-Spikes dominiert [Sei56]. In Simulationen konnte auf der Längenskala und Lebensdauer von Thermal-Spikes ein hochgradig kollektives atomares Verhalten beobachtet werden, das ähnlich den Gesetzen der makroskopischen Hydrodynamik viskose Effekte hervorruft, die das System lokal in den thermodynamischen Gleichgewichtszustand überführen und Relaxation von mechanischen Spannungen [May03, May03a] sowie ein Glätten der Oberfläche [May01, Umb01] zur Folge haben. 
Ein Teil der vorliegenden Arbeit konzentriert sich auf die morphologische Entwicklung der inneren Grenzfläche in dem selbst in flüssiger Phase nicht mischbaren System Ni-Ag unter Ionenbestrahlung [Pet05]. Frühere Arbeiten an Multilagen des thermodynamisch vergleichbaren Systems Fe-Ag indizieren durch Schärfung der Satellitenpeaks des Röntgendiffraktogramms eine Abnahme der Grenzflächenrauigkeit während der Bestrahlung [Kre95]. Die hier angestellten experimentellen Untersuchungen der fluenzabhängigen Mikrostruktur- sowie Rauigkeitsentwicklung an einer Ni-Ag-Phasengrenze sollen die diesbezüglich von Mayr durchgeführten Simulationen [Pet05] komplementieren und eine Identifikation der auf atomarer Ebene dominierenden Mechanismen ermöglichen (Kapitel 3).

Strukturierungsverfahren werden neben der Glättung von Grenzflächen vor allem zur Erzeugung geordneter Oberflächenstrukturen eingesetzt. Ordnung ist ein zwingender Faktor für eine effiziente Adressierung funktionaler Bausteine in der Nanotechnologie. Im Zuge der Miniaturisierung zur Erhöhung der Leistungsdichte magnetischer und elektronischer Elemente werden immer kleinere Strukturen erforderlich, sodass zeit- und kostenaufwendige Strukturierungsverfahren wie die optische oder die Elektronenstrahllithographie in absehbarer Zeit an ihre Grenzen stoßen. Selbstorganisationsprozesse ${ }^{1}$ haben daher in den letzten Jahren verstärktes Interesse geweckt [Ros01], da sie potenziell eine gezielte Anordnung von Strukturen auf deutlich reduzierter Skala ermöglichen und diese Eigenschaft mit einer üblicherweise einfachen Prozessaktivierung verbinden. Ihre technische Anwendung ist jedoch infolge mangelnder Fernordnung häufig limitiert. Diese mangelnde Fernordnung lässt sich durch eine Vorstrukturierung beheben. Viel versprechend sind Selbstorganisationsprozesse in Verbindung mit einer zusätzlichen Strukturierung, wobei sich die resultierenden Strukturen auf kleiner Längenskala durch Selbstorganisation anordnen, während sie mit speziellen, vorher eingeprägten Oberflächenmerkmalen wechselwirken, die auch auf größerer Längenskala variieren können. Zudem besteht die Möglichkeit mittels spezieller Oberflächengeometrien den Selbstorganisationsprozess gezielt zu steuern. Ähnlich kombinierte Prozesse wurden beispielsweise unter Verwendung selbstanordnender organischer Moleküle zur Reduzierung der Breite lithographisch hergestellter Drähte angewandt [Hat01] oder zur Erzeugung von Nanodrähten vertikaler Wachstumsrichtung mittels elektrochemischer Deposition in die hexagonal angeordneten Nanoporen anodisch oxidierten Aluminiums eingesetzt [Str99].

Ein wesentlicher Aspekt dieser Arbeit bezüglich der Untersuchung gesteuerter Selbstorganisationsprozesse, deren Pfad durch eine spezielle Oberflächengeometrie eingegrenzt wird, bestand zunächst in der Herstellung geeignet strukturierter Substrate. Seit der Pionierarbeit von Navez et al. [Nav62] ist bekannt, dass der Ionenbeschuss von Festkörperoberflächen unter schrägem Einfall zur Ausbildung parallel ausgerichteter periodischer Wellenstrukturen mit Wellenlängen zwischen etwa 10nm und einigen Mikrometern führen

\footnotetext{
${ }^{1}$ Selbstorganisation ist die autonome Organisation von Komponenten in Mustern oder Strukturen ohne menschliche Intervention [Whi02].
} 
kann, die das Resultat konkurrierender kinetischer Oberflächenprozesse sind [Bra88]. Seither stand die Dynamik dieser Instabilität im Mittelpunkt einer Vielzahl von experimentellen sowie theoretischen Studien [Cha07]. Hier wurden nun in Hinblick auf die Anwendung als Templates Untersuchungen dahingehend geführt, die aus technischer Sicht relevante laterale Ordnung dieser Rippel zu optimieren (Kapitel 4), um den Einfluss entsprechend strukturierter Oberflächen auf unterschiedliche Selbstorganisationsprozesse zu erforschen.

Zur Aktivierung eines Selbstorganisationsprozesses sind mehrere Verfahren denkbar. Meist handelt es sich um einen Energieeintrag - sei es nun elektrisch, magnetisch, thermisch oder durch Impulsübertragung - in ein System, das sich fern des thermodynamischen Gleichgewichts befindet. Ferner besteht die Möglichkeit, einem System energetische Teilchen hinzuzufügen, sodass die Materie selbst die benötigte Energie mitbringt. Hier soll anhand des stark entmischenden Systems Ni-Ag untersucht werden, inwieweit eine wellenförmige Topographie der Substratoberfläche infolge lokaler Krümmungsgradienten die Mechanismen der Kristallorganisation sowie den zu erwartenden Separationsprozess während der Deposition entsprechender Legierungsschichten dahingehend beeinflusst, dass eine geometrische Ordnung erzielt wird (Kapitel 5).

Den Schwerpunkt bildet schließlich die Selbstorganisation durch thermisch aktivierte Entnetzung metallischer Schichten in fester Phase [Pet08] (Kapitel 6). Bislang wurde die Entnetzung metallischer Filme in der Nanotechnologie als störendes Resultat zu hoher thermischer Belastung gesehen, welche häufig eine irreversible Schädigung elektronischer Bauteile zur Folge hat. Viele Untersuchungen wurden daher mit dem Ziel geführt, die thermische Stabilität metallischer Leitungen auf dielektrischen Substraten in Hinblick auf die Zuverlässigkeit integrierter Schaltkreise oder von Funktionsschichten zu erhöhen [Hum81, Alf03, Kra03]. Die Entnetzung kann indes auch als Selbstorganisationsprozess zur Nanostrukturierung genutzt werden. Das hohe Oberflächen-zu-Volumen-Verhältnis dünner Filme resultiert in einer starken treibenden Kraft zur Oberflächenreduzierung und ermöglicht durch thermische Aktivierung den Umwandlungsprozess in isolierte Inseln. Die Initiierung des Agglomerationsprozesses polykristalliner Filme wird, gestützt durch thermodynamische Gleichgewichtsbetrachtungen der auftretenden Grenzflächenspannungen von Mullins [Mul57] sowie Srolovitz und Safran [Sro86], weitestgehend auf die Furchenbildung an Korngrenzen zurückgeführt. Entnetzung ist jedoch ein kinetischer Prozess und der genaue Pfad der morphologischen Entwicklung wird maßgeblich durch die operierenden Transportmechanismen bestimmt. Saxena et al. [Sax05] haben basierend auf experimentellen Daten bezüglich der Aktivierungsenergie entnetzender Kupferschichten kürzlich ein Modell vorgeschlagen, welches die Grenzflächendiffusion zwischen Schicht und Substrat als entscheidenden Transportmechanismus hinsichtlich der Kinetik der Agglomeration beinhaltet. Während phänomenologische Modelle meist der Oberflächendiffusion die tragende Rolle zusprechen [Bra66, Jir90], sind die experimentellen Daten diesbezüglich nicht eindeutig und die genaue Natur des Entnetzungsvorgangs geschlossener Filme nicht im Detail geklärt. 
Für eine gezielte Steuerung von Selbstorganisationsprozessen zur kontrollierten Herstellung von Nanostrukturen ist eine genaue Kenntnis der dem Prozess zugrunde liegenden Mechanismen unabdingbar. Hier sollen zunächst mittels topographischer Abbildungsverfahren und Widerstandsmessungen die Phasen der morphologischen Entwicklung sowie die Kinetik speziell in Hinblick auf den kinetisch limitierenden Prozess der Entnetzung an dünnen Nickelschichten auf glattem Substrat untersucht werden. Auf Grund des Anwendungspotentials beispielsweise zur Erzeugung isolierter Nanopartikel für die katalytische Synthese von Kohlenstoffnanoröhren [Iij93, Kak06, Ran07], bei der eine Größenselektion mit schmaler Verteilung der Partikeldurchmesser und regelmäßiger Anordnung wünschenswert ist, sind die hier erstmals durchgeführten detaillierten Studien der Agglomeration von Nickel zudem von besonderem technischen Interesse.

Anschließend soll das Entnetzungsverhalten entsprechender Nickelschichten auf rippelstrukturierten Substraten analysiert werden, wobei speziell der Frage nachgegangen wird, ob über die zugrunde liegende Oberflächenstruktur eine gezielte Beeinflussung des Selbstorganisationsprozesses hinsichtlich der Ausbildung eines Feldes von Nanostäben oder Nanodrähten induziert werden kann. Abschließendes Ziel ist die Charakterisierung des Einflusses einer nicht mischenden Komponente auf den Entnetzungsprozess anhand kodeponierter NiAg-Schichten auf glattem sowie rippelstrukturiertem Substrat. 


\section{Kapitel}

\section{Experimentelles}

In diesem Kapitel werden die Methoden und Techniken zur Herstellung und Charakterisierung verwendeter Proben erläutert, wobei zunächst auf den Herstellungsprozess der metallischen dünnen Schichten eingegangen wird und anschließend eine Erläuterung der angewendeten Charakterisierungsmethoden folgt. Der Rippelbildung durch Ionenbestrahlung und Entwicklung geeignet strukturierter Substrate ist ein eigenes Kapitel gewidmet.

\subsection{Präparation metallischer dünner Schichten}

Die experimentellen Untersuchungen bezüglich Glättung innerer Ni-Ag-Grenzflächen sowie der Selbstorganisation dünner Ni- und NiAg-Filme beinhalten unterschiedliche Herstellungsverfahren, bei denen sowohl die physikalische Gasphasenabscheidung mittels Elektronenstrahlverdampfens, als auch die Sputterdeposition zum Einsatz kamen. Grundlegend für die Studien des Selbstorganisationsprozesses ist eine genaue Temperaturkontrolle, da die Erhöhung der thermischen Energie als Aktivierungsmechanismus und treibende Kraft für die Agglomeration fungiert.

\subsubsection{Herstellung aufgedampfter Schichten}

\subsubsection{Die Ultrahochvakuumanlage}

Speziell für die Strukturbildung während der Entnetzung dünner Schichten, die getrieben wird durch die Minimierung auftretender Grenzflächenspannungen, spielt eine verunreinigungsfreie Oberfläche eine entscheidende Rolle. Sämtliche aufgedampften Ni-, Ag- und NiAg-Schichten wurden in einer Ultrahochvakuumanlage (UHV-Anlage) präpariert (Abbildung 2.1), deren Grundgerüst von der Firma VARIAN hergestellt und deren struktureller Aufbau beispielsweise in den Dissertationen von B. M. Reinker [Rei96] und H. Geisler [Gei96] genauer beschrieben wurde. Im Folgenden soll nur auf die für diese Arbeit 
relevanten Details der Apparatur eingegangen werden. Sie besteht aus einer Schleusenkammer, in der mittels Turbomolekularpumpe ein Druck von $1 \cdot 10^{-8}$ mbar erreicht wird, einer Präparationskammer mit drei simultan betreibbaren Elektronenstrahlverdampfern ${ }^{2}$ und Heiz- / Kühlvorrichtung für den Probenhalter, sowie einer Analysekammer, in die ein Rastertunnelmikroskop ${ }^{3}$ und eine differentiell gepumpte, rasterfähige Ionenquelle ${ }^{4}$ integriert sind, wobei der Probentransport zwischen den einzelnen Kammern durch ein Magnetstangentransfersystem ermöglicht wird. Der Druck in der Präparations- und Analyse-

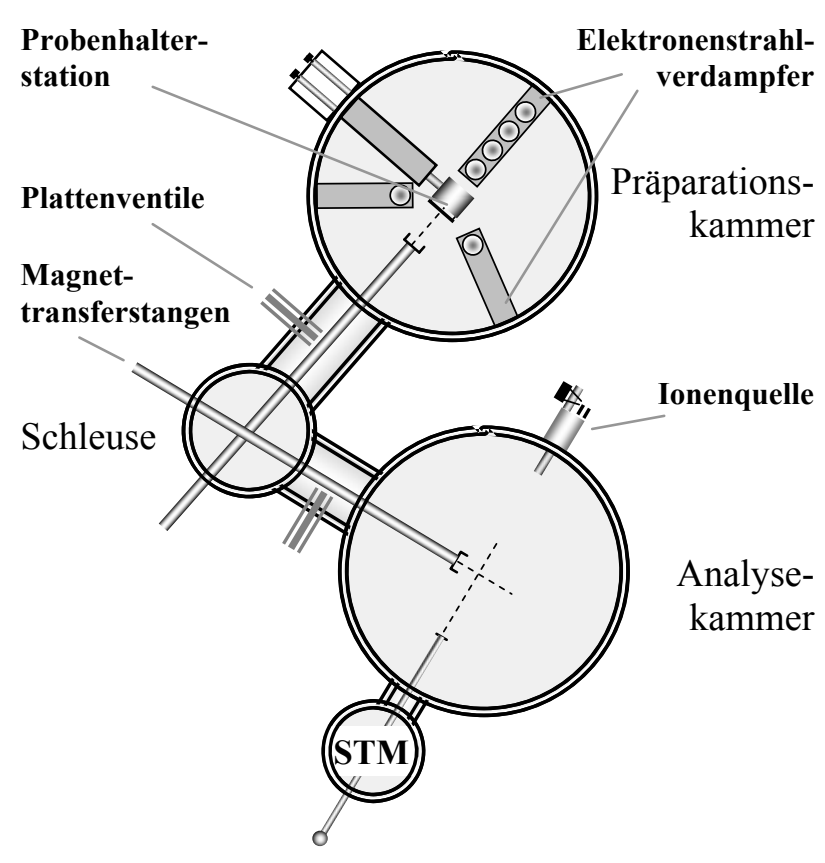

Abbildung 2.1: Aufbau der UHV-Anlage. kammer wird mittels Ionengetterpumpen nach Ausheizen auf $2 \cdot 10^{-10}$ mbar gehalten.

Auf Grund eines Defekts der in die Probenhalterstation eingebetteten Heizwendel innerhalb der Präparationskammer wurde ein neues Heizelement konstruiert (Abbildung 2.2). Kernstück dieser Vorrichtung sind zwei in Reihe geschaltete, mit pyrolytischem Bornitrid überzogene Widerstandsheizelemente der Firma BORALECTRIC ${ }^{\circledR}$, die zusammen eine maximale Heizleistung von $600 \mathrm{~W}$ besitzen. Um einen ausreichenden thermischen Kontakt der kühlbaren Probenhalterstation und der Heizelemente zum Probenteller zu gewährleisten, wurden isolierende Hohlräume vermieden und die Heizelemente in entsprechend ausgesparte Graphitplatten eingebaut. Hierbei wurde neben der hohen Temperaturbeständigkeit zum einen die spezielle Wärmeleitfähigkeit des Graphits ausgenutzt, die deutlich höher ist als bei vielen Metallen, und zum anderen seine geringe Dichte, um eine zu starke mechanische Beanspruchung der aus dünnem Molybdänblech gefertigten Probenhalterstation zu verhindern. Der Kontakt zwischen Heizeinsatz und Probenteller wird durch zwei an der Rückseite des Probenhalters angeschraubte Kupferbleche hergestellt. Dies stellt eine Einschränkung für die Effizienz der Heizung dar, gewährleistet jedoch auch weiterhin die Rotationsmöglichkeit des Probenhalters während des Aufdampfprozesses. Das Graphit wird von einer dünnen Mo-

\footnotetext{
${ }^{2}$ LEYBOLD-HERAEUS ESV4

${ }^{3}$ STM 1 der Firma OMICRON

${ }^{4}$ IQE 12/38 der Firma SPECS ${ }^{\circledR}$
} 


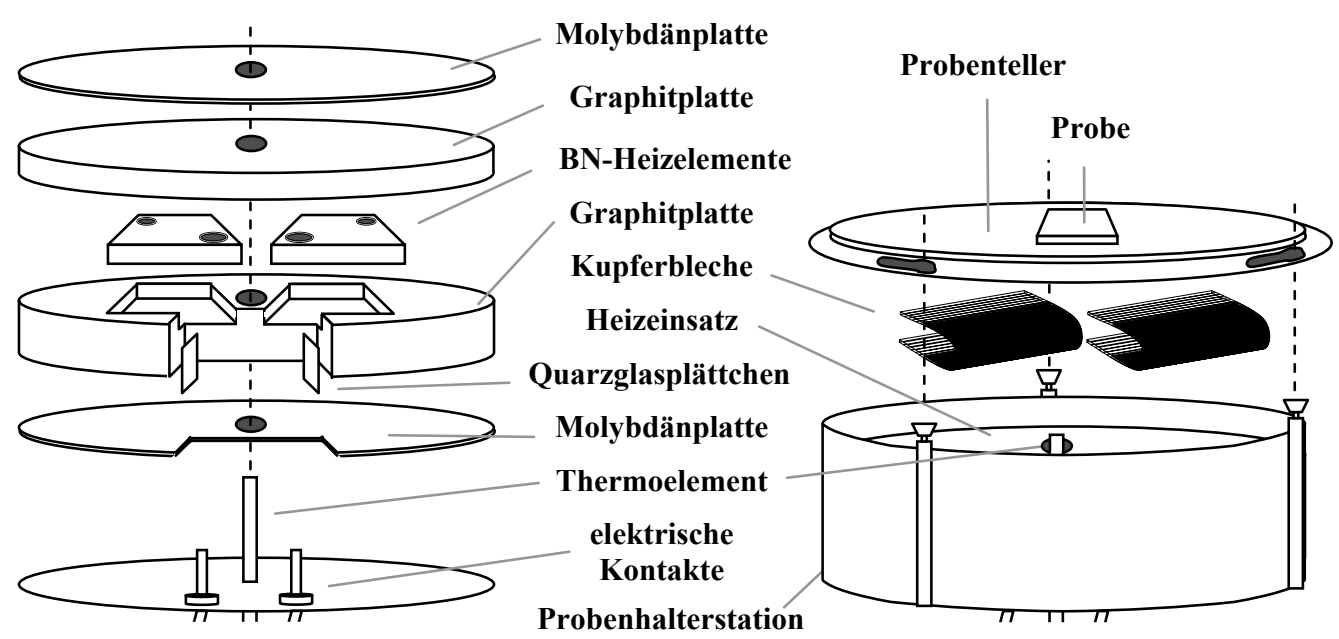

Abbildung 2.2: Schematische Darstellung des konstruierten Heizeinsatzes (links) und dessen Integration in die Probenhalterstation (rechts).

lybdänplatte abgedeckt, wodurch eine glatte Oberfläche gebildet wird, die vor starkem Abrieb durch die Kupferblechkontakte geschützt ist.

\subsubsection{Temperatureichung}

Infolge der Rotationsmöglichkeit und des Schleusprinzips ist eine permanente Vorrichtung für die Installation eines Thermoelements auf dem Probenhalter nicht möglich. Ein Thermoelement ist innerhalb des Heizeinsatzes fest integriert, und mittels eines temporären Thermoelements auf dem Probenteller wurde eine entsprechende Eichkurve für die Probentemperatur erstellt (Abbildung 2.3). Auf Grund der Trägheit des Systems bezüglich Wärmeleitung und der Notwendigkeit einer genauen Temperatursteuerung bei der thermischen Behandlung der Probenserien wurde ein computergesteuerter PID-Regler verwendet und dahingehend konfiguriert, eine maximale Heizrate zu erzielen, gleichzeitig aber ein starkes Überschreiten des Temperatursollwertes zu vermeiden. Abbildung 2.4 zeigt den Temperaturverlauf während einer repräsentativen Eichmessung. Um die stationäre Gleichgewichtstemperatur auf dem Testsubstrat (Probentemperatur) von $290^{\circ} \mathrm{C}$ während des Aufheizvorgangs möglichst schnell zu erreichen, wurde die Temperatur des Heizeinsatzes so gesteuert, dass sie den Sollwert etwas überschreitet. Hierdurch konnte eine maximale mittlere Heizrate von $10^{\circ} \mathrm{C} / \mathrm{min}$ erreicht werden, wobei die Probentemperatur ihren Zielwert nur kurzzeitig um etwa $2^{\circ} \mathrm{C}$ übersteigt. Der Vergleich mit einer Eichkurve des ursprünglichen Heizkonzeptes (siehe [May00]) zeigt, dass die Heizwirkung an der Probe durch die neue Konstruktion deutlich verbessert werden konnte. 

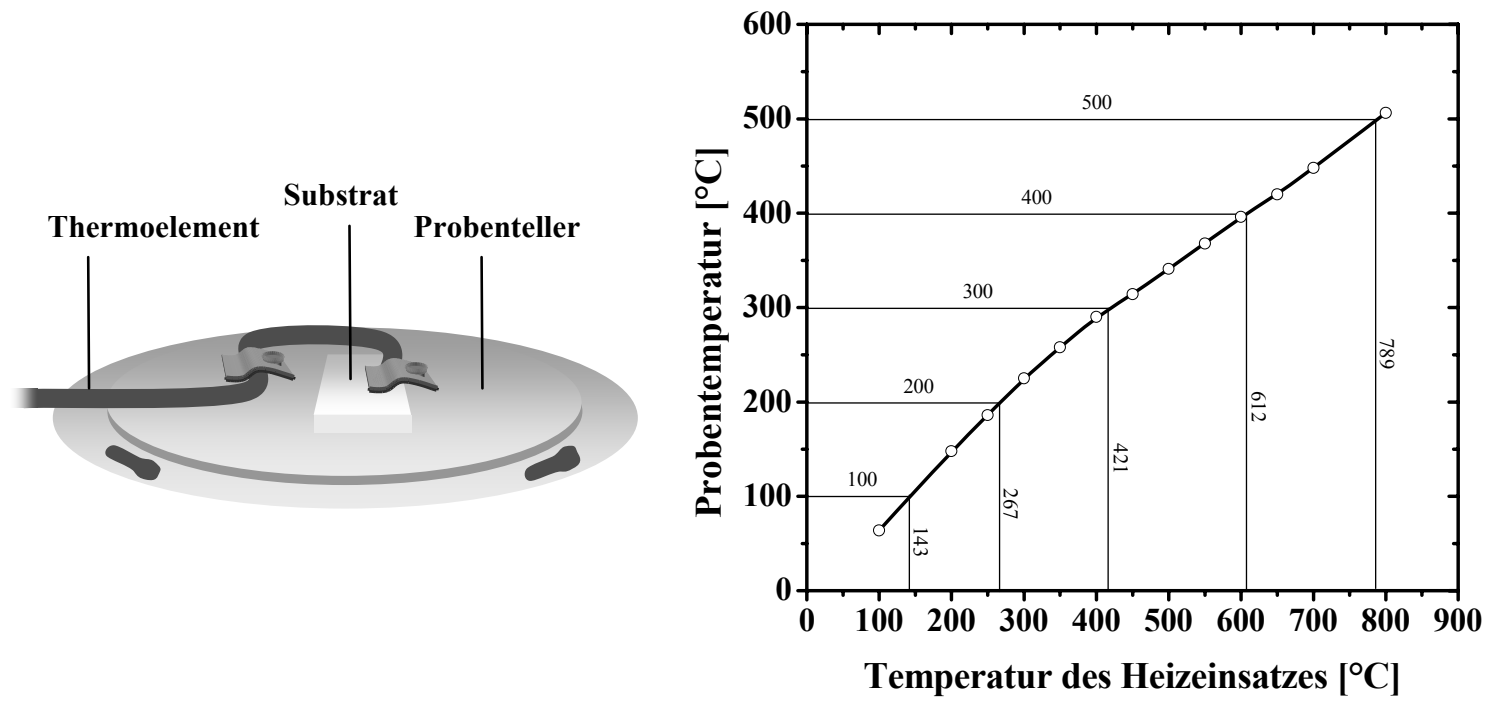

Abbildung 2.3: Temperatureichkurve (rechts) und Position des Thermoelementes während der Eichmessung (links).

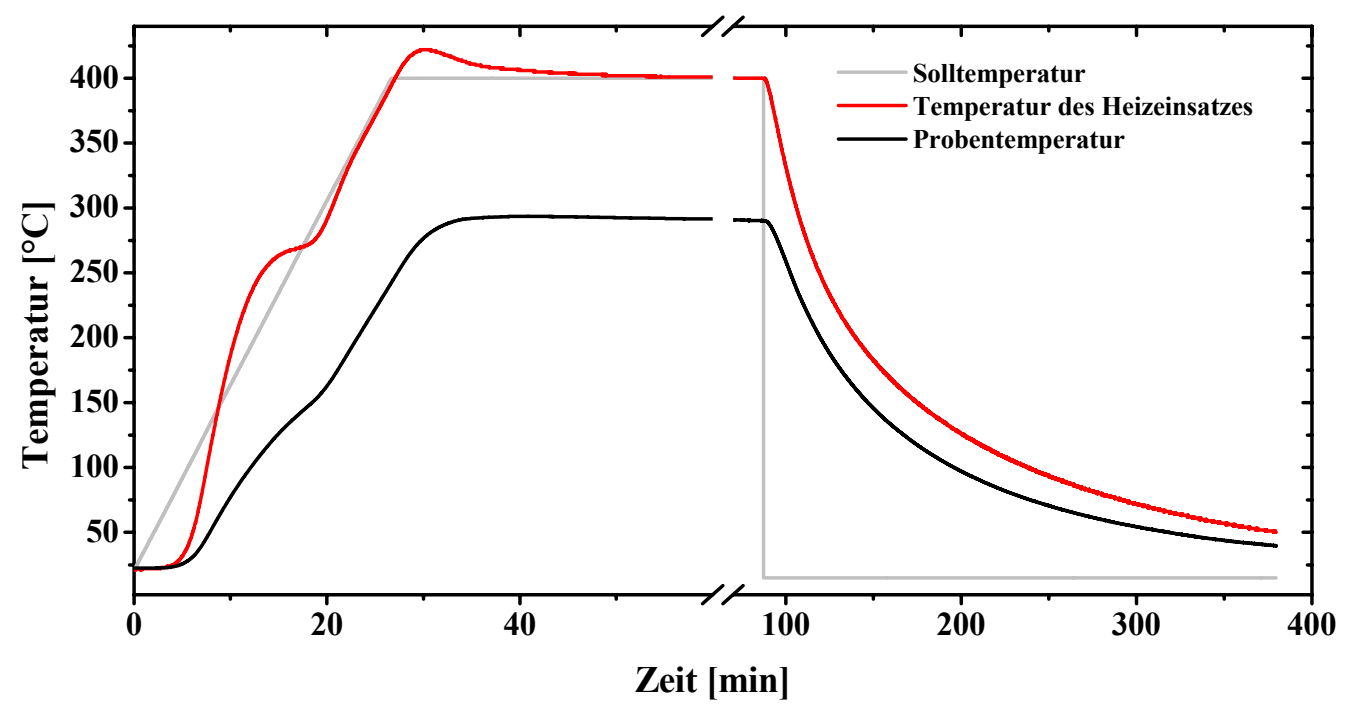

Abbildung 2.4: Heiz- und Abkühlverhalten des Heizeinsatzes und des Probentellers. 


\subsubsection{Deposition}

Die metallischen Schichten ${ }^{5}$ wurden mittels Elektronenstrahlverdampfens auf einseitig poliertes, thermisch oxidiertes Silizium mit einer Oxidschichtdicke von etwa 500nm deponiert, wobei die Oxidschicht zur Vermeidung von Interdiffusion zwischen Schicht und Substrat dient. Die Substrate wurden zuvor in Aceton und Isopropanol gereinigt, mit einem Heißluftgerät getrocknet, um Lösungsmittelrückstände und Adsorbate auf der Oberfläche zu vermeiden, und mit Stickstoff abgeblasen. Die Reinigung in Aceton bzw. Ethanol ohne nachträgliche Behandlung mit Isopropanol erwies sich als nicht hinreichend und offenbarte schlierenartige Rückstände unter dem Rasterelektronenmikroskop.

Für die Untersuchungen der Grenzflächenrauigkeit im System Ni-Ag wurden entsprechende Doppellagen unter Verwendung der Substratkühlung hergestellt, um eine feinere Mikrostruktur, d.h. geringere Korngrößen, zu erzielen. Der Kühlmechanismus beruht auf einem Durchfluss von flüssigem Stickstoff durch die Kühlleitung der Probenhalterstation. Mit einem Überdruck von 0.2 bar hat sich nach etwa 2 Stunden eine Gleichgewichtstemperatur von $-75^{\circ} \mathrm{C}$ eingestellt. Zur Minimierung verstärkter Restgaskondensation auf dem kalten Substrat wurden zuvor die integrierten Kühlfallen mit flüssigem Stickstoff befüllt.

Sämtliche Schichten wurden bei einem Basisdruck von etwa $10^{-10}$ mbar und mittels Ratensteuerung über Schwingquarze ${ }^{6}$ mit einer konstanten Depositionsrate von $0.1 \mathrm{~nm} / \mathrm{s}$ bzw. $0.5 \mathrm{~nm} / \mathrm{s}$ für die Doppellagen hergestellt. Anhand von Röntgenreflektivitätsmessungen (Abschnitt 2.4) erfolgte eine regelmäßige Kontrolle der tatsächlich deponierten Schichtdicke.

\subsubsection{Herstellung gesputterter Schichten}

\subsubsection{Die Sputterdeposition}

Die Deposition mittels Magnetron-Sputterns (Abbildung 2.5) basiert auf dem Zerstäuben eines aus dem zu deponierenden Material gefertigten Targets. Das Anlegen einer Spannung zwischen Target und Anode löst Elektronen aus dem Target und resultiert in der Ionisation von in die Kammer eingelassenen Argongasatomen. Permanentmagnete vermitteln hierbei ein Magnetfeld, dass die Elektronen und $\mathrm{Ar}^{+}$-Ionen auf eine spiralförmige Bahn lenkt und somit die Ionisationswahrscheinlichkeit erhöht, sodass ein stabiles Plasma entsteht. Durch Stöße der auf das Target beschleunigten Ionen werden Oberflächenatome herausgelöst,

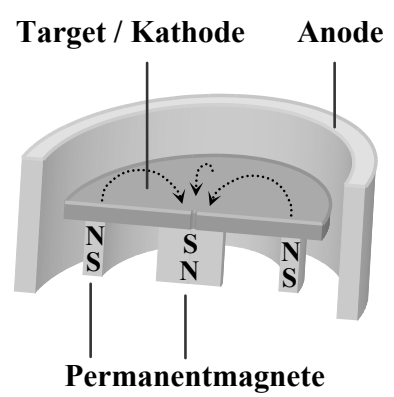

Abbildung 2.5: Querschnitt einer Magnetronsputterquelle.

\footnotetext{
${ }^{5}$ Verwendete Materialien: Ni mit einer Reinheit von $99.997 \%$ und Ag mit einer Reinheit von $99.99 \%$

${ }^{6}$ XTC der Firma LEYBOLD / INFICON
} 
die sich auch auf dem Substrat niederschlagen. Die spiralförmige Bahn der Ionen führt zu einem inhomogenen Abtrag des Targets präferentiell entlang des auftretenden Plasmaringes, sodass sich eine Erosionsspur ausbildet.

\subsubsection{Gesputterte Ni-Schichten}

Zur Untersuchung der Kinetik des Entnetzungsprozesses von $\mathrm{Ni}$ auf $\mathrm{SiO}_{2}$ wurde ein in situVierpunkt-Widerstandsmessaufbau (siehe Abschnitt 2.2) entwickelt und zusammen mit einem Ni-Target ${ }^{7}$ in eine Sputteranlage integriert. Die Sputteranlage besitzt einen ArDurchflussregler sowie ein elektronisch gesteuertes Schmetterlingsventil, womit die Pumpleistung der Turbomolekularpumpe gezielt reduziert werden kann. Bei maximaler Pumpleistung wird ein Basisdruck von $5 \cdot 10^{-7}$ mbar erreicht. Vor jeder Deposition wurde das Target zur Beseitigung des Oberflächenoxids und Adsorbaten für 5 Minuten vorgesputtert. Es wurden sowohl $5 \mathrm{~nm}$ Ni-Schichten mit einer Leistung von $10 \mathrm{~W}$ bei $305 \mathrm{~V}$ und einer Depositionsrate von $2.2 \mathrm{~nm} / \mathrm{min}$, als auch $100 \mathrm{~nm}$ Ni-Kontakte mit $50 \mathrm{~W}$ bei $320 \mathrm{~V}$ und $10.7 \mathrm{~nm} / \mathrm{min}$ bei einem Ar-Gasdruck ${ }^{8}$ von $1 \cdot 10^{-3}$ mbar gesputtert. Die Depositionsrate wurde hierbei durch entsprechende Schichtdickenbestimmung mittels Röntgenreflektometrie regelmäßig kontrolliert.

\subsubsection{Gesputterte NiAg-Schichten}

Um den Einfluss von Silber auf das Entnetzungsverhalten der Ni-Schichten sowie die Selbstorganisation während der Deposition beider Legierungskomponenten zu analysieren, wurden kogesputterte NiAg-Schichten präpariert. Der Aufbau der hierbei eingesetzten Ultrahochvakuumanlage ist in den Dissertationen von B. Sass [Sas04] und F. Leuenberger [Leu04] sowie der Diplomarbeit von C. Tusche [Tus03] näher beschrieben. Sie besteht unter anderem aus einer Depositionskammer mit integrierter Magnetronsputterquelle und heizbarem Probenträger, einem Schleusentransfersystem sowie einer Analysekammer mit Röntgen-Photoelek-

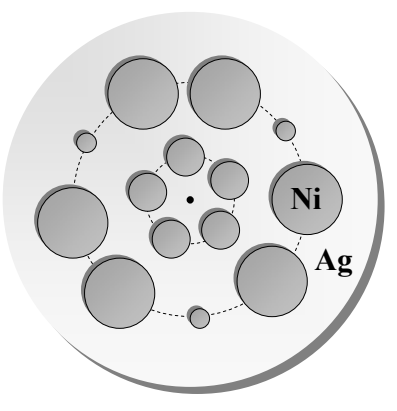

Abbildung 2.6: Schematische Darstellung des NiAg-Targets. tronenspektrometer und Ionenquelle mit Rastereinheit zur Oberflächen- bzw. Tiefenanalyse.

\footnotetext{
${ }^{7}$ Mittels Funkenerosion angepasstes Nickelblech mit einer Reinheit von 99.98\%

${ }^{8}$ Argongas mit einer Reinheit von $99.999 \%$
} 
Zur gleichzeitigen Deposition von Ni und Ag in einem Stöchiometrieverhältnis von 1:1 wurde ein Ni-Target ${ }^{9}$ mit einer partiell aufgebohrten Silberfolie ${ }^{10}$ belegt (Abbildung 2.6). Auf Grund des inhomogenen und verstärkten Abtrags entlang der Erosionsspur lassen sich die benötigten Flächenanteile der Elemente nicht allein anhand des Sputter-Yields bestimmen. Zwecks Kompositionsjustierung wurden daher Filme von $1 \mu \mathrm{m}$ Dicke deponiert, mit Hilfe von energiedispersiver Röntgenspektroskopie (Abschnitt 2.7) analysiert und die Bohrungen sukzessiv erweitert. Die gesputterten Schichten zeigen unter Verwendung des fertig gestellten Targets eine Atomkonzentration von 47.5(3)\% Ni und 52.5(4)\% Ag.

Es wurden NiAg-Schichten unterschiedlicher Dicke bei einem Basisdruck von $10^{-9}$ mbar und einem Argongasdruck ${ }^{8}$ von $10^{-2}$ mbar hergestellt. Mit $30 \mathrm{~W}$ Leistung bei $800 \mathrm{~V}$ beträgt die Depositionsrate hier jeweils $20 \mathrm{~nm} / \mathrm{min}$. Eine Deposition bei erhöhten Substrattemperaturen hat durch das Ausgasen von Verunreinigungen des eingeschleusten Probenträgers einen entsprechend höheren Basisdruck zur Folge. Um diesen Effekt zu reduzieren, wurde der Probenträger zuvor für zwei Stunden bei $100^{\circ} \mathrm{C}$ oberhalb der jeweiligen Depositionstemperatur gehalten. Das Target wurde hier ebenfalls vor jedem Depositionsprozess für 5 Minuten vorgesputtert.

\subsection{Widerstandsmessungen}

Strukturelle und chemische Modifikationen führen zur Veränderung der Elektronenstreuung speziell in dünnen metallischen Schichten und resultieren somit in der Variation des elektrischen Widerstandes. Die Installation einer "in situ"-Widerstandsmessung in die Ultrahochvakuumanlage, die zum Aufdampfen von Ni-Schichten verwendet wurde (Abschnitt 2.1.1.1), war in Anbetracht des Schleusprinzips und des drehbaren Probenhalters nicht möglich. "in situ" ist jedoch auf Grund der hohen Affinität von Nickel zu Sauerstoff und der Signifikanz der Oberflächeneigenschaften während des Entnetzungsprozesses eine entscheidende Anforderung. Die Entwicklung und Integration eines Vierpunktwiderstandsmessaufbaus in die in Abschnitt 2.1.2.2 vorgestellte Sputteranlage ermöglicht eine entsprechende Aufzeichnung des elektrischen Widerstandes in Abhängigkeit von der Temperatur und erlaubt anhand unterschiedlicher Heizraten Rückschlüsse auf die Kinetik des Entnetzungsprozesses. Der schematische Aufbau ist in Abbildung 2.7 dargestellt.

Mittels PID-geregelter Strahlungsheizung in Form von einer abgeschirmten 400W Projektorbirne unterhalb des Substrats lassen sich konstante Heizraten weit über $20^{\circ} \mathrm{C} / \mathrm{min}$ erreichen. Kontaktierung und Schichtdeposition erfordern eine zweistufige Präparationsprozedur. In einem ersten Sputtervorgang wurden durch Schattenmaskenverfahren elektrische Leitungen einer Dicke von 100nm deponiert, die zur Vermeidung von chemisch indu-

\footnotetext{
${ }^{9}$ Ni-Target mit einer Reinheit von $99.98 \%$

${ }^{10}$ Ag-Folie mit einer Reinheit von $99.99 \%$
} 


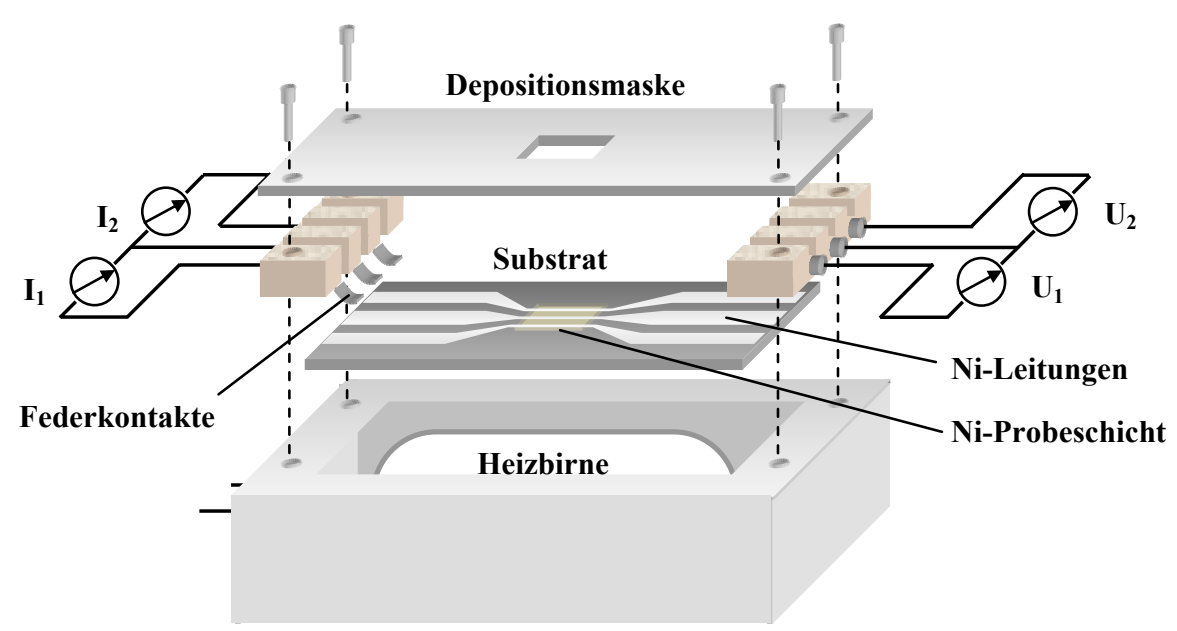

Abbildung 2.7: Schematische Darstellung des 2-Kanal-Widerstandsmessaufbaus.

zierten Widerstandsänderungen in der Probeschicht ebenfalls aus Nickel hergestellt wurden. Die Zuleitungen wurden mit Federkontakten elektrisch kontaktiert und ein Thermoelement direkt auf dem Substrat platziert. Anschließend wird in einem zweiten Sputtervorgang die zu analysierende $5 \mathrm{~nm}$ dicke Ni-Probeschicht durch die Depositionsmaske aufgebracht.

Da die Federkontakte auf Grund thermischer Ausdehnung bei höheren Temperaturen fehleranfällig sind und es folglich zu Sprüngen im Widerstand kommen kann, wurde die Geometrie des Aufbaus so gewählt, dass sie eine 2-Kanal-Messung mit einer sequentiellen Ansteuerung der einzelnen Kanäle erlaubt. Hierdurch lassen sich Messartefakte über den Vergleich beider Signale im Wesentlichen eliminieren. Das Messprinzip beruht auf einer herkömmlichen Vierpunktmessung. Die Steuerung erfolgt über eine mittels LABVIEW ${ }^{\circledR}$ entwickelten Software, wobei ein Strompuls von $500 \mu \mathrm{A}$ abwechselnd auf die beiden Kanäle geschaltet und simultan an den gegenüberliegenden Enden der Ni-Leitungen die Spannung abgefragt wird. Strominduzierte Effekte in der Probeschicht können bei der geringen resultierenden Stromdichte von etwa $10^{3} \mathrm{~A} / \mathrm{cm}^{2}$ ausgeschlossen werden. Das Einsetzen von Elektromigration wird im Allgemeinen erst bei Stromdichten oberhalb von $10^{6} \mathrm{~A} / \mathrm{cm}^{2}$ beobachtet [Ame00].

\subsection{Röntgendiffraktometrie (XRD)}

Zur Strukturanalyse der Schichten mittels Röntgendiffraktometrie wurde das Zweikreisdiffraktometer D8 der Firma BRUKER AXS in Bragg-Brentano-Geometrie eingesetzt, das mit einer $\mathrm{Cu}$-Röhre der Wellenlänge $\lambda=0.154 \mathrm{~nm}\left(\mathrm{Cu}-\mathrm{K}_{\alpha}\right.$-Linie) betrieben wurde. Kristalle besitzen durch die strenge Atomverteilung auf Gitterplätze eine langreichweitige Trans- 
lationssymmetrie. Unter dem Ausfallswinkel $2 \theta$ kommt es durch elastische Streuung an parallelen Netzebenen mit dem Abstand d unter der Bragg-Bedingung

$$
\mathrm{n} \lambda=2 \mathrm{~d} \sin \theta
$$

zur konstruktiven Interferenz. $\mathrm{n}$ bezeichnet hierbei die Ordnung des Interferenzmaximums. Infolge der regelmäßigen Atomanordnung entstehen im Beugungsbild kristalliner Materialien scharfe Reflexe, deren Halbwertsbreite $\Gamma$ abhängig von der Größe der kohärent streuenden Bereiche ist. Unter der Annahme kugelförmiger Kristallite lässt sich über die Scherrer-Formel [Cul78] der Kristalldurchmesser $\tau$ abschätzen:

$$
\tau=\frac{0.9 \lambda}{\Gamma \cos \theta}
$$

\subsection{Röntgenreflektometrie (XRR)}

Röntgenreflektometrie bietet die Möglichkeit der Schichtdicken- und Rauigkeitsbestimmung dünner Filme. Bei bekannter Stöchiometrie ist außerdem eine Bestimmung der Dichte möglich. Wie bei der Röntgendiffraktometrie wurde hierfür das Zweikreisdiffraktometer D8 der Firma BRUKER AXS in spekulärer Geometrie (Einfallswinkel $=$ Ausfallswinkel) verwendet. Schichtdicken und Rauigkeiten wurden mit Hilfe des Programms REFSIM 2.0 (ebenfalls von BRUKER AXS) ermittelt, indem der theoretische winkelabhängige Verlauf der Reflektivität basierend auf dem Névot-Croce-Modell [Nev80] und dem Parratt-Formalismus [Par54] an entsprechende Messdaten angefittet wurde.

Das Prinzip der Reflektometrie beruht auf der Reflexion der Strahlung an Probenoberfläche und innerer Grenzflächen, hervorgerufen durch den Unterschied in der Elektronendichte der Schichten, die einem Unterschied im Brechungsindex entspricht. Der einfallende Röntgenstrahl mit dem Wellenvektor $\left|\overrightarrow{\mathrm{k}}_{0}\right|=2 \pi / \lambda$ wird an den Elektronen der Atome mit

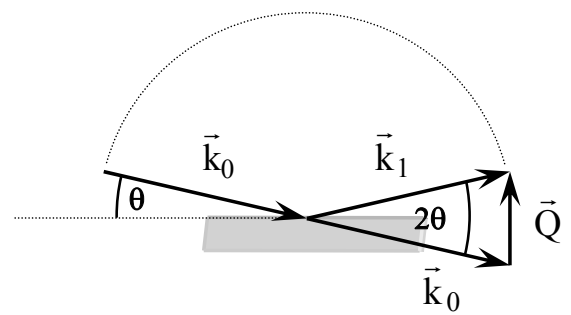

Abbildung 2.8: Spekuläre Röntgenstreuung. 
dem Streuvektor $\overrightarrow{\mathrm{Q}}=\overrightarrow{\mathrm{k}}_{1}-\overrightarrow{\mathrm{k}}_{0}$ elastisch gestreut (Abbildung 2.8). Die Wellenlänge der Strahlung liegt üblicherweise im Wellenlängenbereich um $0.1 \mathrm{~nm}$ (hier $\lambda\left(\mathrm{Cu}-\mathrm{K}_{\alpha}\right)=$ $0.154 \mathrm{~nm})$, wobei bei kleinen Winkeln $2 \theta \leq 6^{\circ}$ gemessen wird, sodass Streuvektor $|\overrightarrow{\mathrm{Q}}|$ und Phase $\vec{Q} \cdot \vec{r}$ ebenfalls klein sind. Die Information rührt folglich von Strukturen der Größenordnung $\geq 1 \mathrm{~nm}$. Kleinere Strukturen im Gitterkonstantenbereich liefern keinen Streukontrast und die genaue Anordnung einzelner Atome spielt keine Rolle. Es genügt also, den komplexen Brechungsindex $n=1-\delta-i \beta$ zu betrachten, dessen Dispersions- und Absorptionsterm sich für Frequenzen weit oberhalb der Resonanzfrequenzen der Elektronen näherungsweise durch $\delta=\lambda^{2} \mathrm{r}_{0} \rho_{\mathrm{e}} /(2 \pi)$ ( $\lambda$ : Wellenlänge, $\mathrm{r}_{0}$ : Bohrscher Atomradius, $\rho_{\mathrm{e}}$ : Elektronendichte) bzw. $\beta=\mu \lambda /(4 \pi)$ ( $\mu$ : linearer Absorptionskoeffizient) ausdrücken lassen [Pre96]. Die reflektierte Intensität kann für eine ideal glatte Grenzfläche zwischen Materie und Vakuum durch den Fresnelschen Reflektionskoeffizienten $r$ als Funktion des Einfallswinkels $\theta$ beschrieben werden [Len92]:

$$
\mathrm{R}=\mathrm{r}^{*} \mathrm{r}=\frac{\left(\theta-\mathrm{p}_{1}\right)^{2}+\mathrm{p}_{2}^{2}}{\left(\theta+\mathrm{p}_{1}\right)^{2}+\mathrm{p}_{2}^{2}}
$$

mit

$$
\begin{aligned}
& \mathrm{p}_{1}^{2}=\frac{1}{2}\left[\sqrt{\left(\theta_{1}^{2}-2 \delta\right)^{2}+4 \beta^{2}}+\left(\theta^{2}-2 \delta\right)\right] \\
& \mathrm{p}_{2}^{2}=\frac{1}{2}\left[\sqrt{\left(\theta_{1}^{2}-2 \delta\right)^{2}+4 \beta^{2}}-\left(\theta^{2}-2 \delta\right)\right] .
\end{aligned}
$$

Ist die Grenzfläche nicht ideal glatt sondern rau, werden die Röntgenstrahlen diffus in verschiedene Richtungen gestreut und die spekuläre Intensität somit abgeschwächt. Die Reflektivität kann hierfür durch Lösen der Helmholtz-Gleichung erhalten werden [Pre96]:

$$
\frac{\partial^{2} \mathrm{E}(\mathrm{z})}{\partial \mathrm{z}^{2}}+\mathrm{k}_{\mathrm{z}}^{2}(\mathrm{z}) \mathrm{E}(\mathrm{z})=0
$$

(E: elektrisches Feld, $z$ : Koordinate senkrecht zur Oberfläche und $k_{z}=k_{0} \sqrt{n^{2}(z)-\cos ^{2} \theta}$ ), wobei der Brechungsindex durch die rauigkeitsinduzierte Dichtevariation in z-Richtung senkrecht zur Schichtebene abhängig von der z-Koordinate ist. Névot und Croce [Nev80] haben für den Verlauf des Brechungsindizes an der Grenzfläche die Form einer Gaußschen Fehlerfunktion angenommen und den Fresnelschen Reflektionskoeffizienten berechnet:

$$
\mathrm{r}_{\mathrm{j}, \mathrm{j}+1}=\frac{\mathrm{k}_{\mathrm{z}, \mathrm{j}}-\mathrm{k}_{\mathrm{z}, \mathrm{j}+1}}{\mathrm{k}_{\mathrm{z}, \mathrm{j}}+\mathrm{k}_{\mathrm{z}, \mathrm{j}+1}} \exp \left(-2 \mathrm{k}_{\mathrm{z}, \mathrm{j}} \mathrm{k}_{\mathrm{z}, \mathrm{j}+1} \sigma_{\mathrm{j}}^{2}\right)=\mathrm{r}_{\mathrm{j}, \mathrm{j}+1}^{\mathrm{glatt}} \exp \left(-2 \mathrm{k}_{\mathrm{z}, \mathrm{j}} \mathrm{k}_{\mathrm{z}, \mathrm{j}+1} \sigma_{\mathrm{j}}^{2}\right) .
$$


Dies ist der allgemeine Ausdruck für Schichtsysteme mit mehreren Grenzflächen. Hierbei bezeichnet $\sigma_{j}$ die Breite der Grenzfläche zwischen der Schicht $j$ und $j+1$ und kann bei strenger Phasentrennung als Rauigkeit identifiziert werden. Dieser Verlauf des Brechungsindizes entspricht einer Gaußschen Verteilung der mittleren Höhe der Grenzfläche. Das Verfahren zur Berechnung der Reflektivität für Schichtsysteme wurde erstmals von Parratt für glatte Grenzflächen durch eine Rekursionsformel formuliert [Par54]:

$$
X_{j}=\frac{R_{j}}{T_{j}}=\exp \left(-2 i k_{z, j} z_{j}\right) \frac{r_{j, j+1}+X_{j+1} \exp \left(2 i k_{z, j+1} z_{j}\right)}{1+r_{j, j+1} X_{j+1} \exp \left(2 i k_{z, j+1} z_{j}\right)}
$$

$X_{j}$ bezeichnet das Verhältnis aus reflektierter $\left(R_{j}\right)$ und transmittierter Amplitude $\left(T_{j}\right)$ der Welle im Medium zwischen den Grenzflächen $\mathrm{j}$ und $\mathrm{j}+1$. Aus Gleichung 2.6 und 2.7 lässt sich dann ausgehend von dem als halbunendlich betrachteten Substrat und der damit verschwindenden reflektierten Amplitude in diesem, sukzessiv die Reflektivität $R=\left|X_{1}^{2}\right|$ von Schichtsystemen errechnen, deren Rauigkeiten klein gegenüber den entsprechenden Schichtdicken sind. Die Schichtdicke $d_{j}=z_{j+1}-z_{j}$ ergibt sich aus der Position der Grenzflächen, die jeweils durch die zugehörige Koordinate $z_{j}$ festgelegt sind.

\subsection{Rasterkraftmikroskopie (AFM)}

Die Rasterkraftmikroskopie stellt eine zur Röntgenstreuung komplementäre Untersuchungsmethode dar, bei der die Oberfläche der Probe im Realraum abgebildet wird. Mit Hilfe statistischer Funktionen lassen sich sowohl Oberflächenrauigkeiten bestimmen als auch spezielle Korrelationen identifizieren, die Rückschlüsse auf physikalische Prozesse bei der Entstehung der Oberflächenmorphologie erlauben.

Entsprechende Messungen wurden mit einem Nanoscope IV AFM der Firma VEECO im Tapping-Mode durchgeführt. Hierbei dringt eine Sonde, bestehend aus einem in Schwingung versetzten Cantilever, an dessen Ende eine Spitze aus Silizium befestigt ist, in das Potential der Probe ein und wird zeilenweise über die Oberfläche gerastert. Mittels eines Rückkopplungsmechanismus bezüglich der Schwingungsamplitude wird somit ein dreidimensionales Profil der Oberfläche aufgezeichnet.

Die mögliche laterale Auflösung des Bildes wird unter Berücksichtigung der Rauigkeit und Krümmung der untersuchten Strukturen hauptsächlich durch die Form und den Krümmungsradius der Spitze bestimmt. Um entsprechende Messungen auszuwerten, ist deshalb die Kenntnis der Sondengeometrie von entscheidender Bedeutung. Abbildung 2.9 zeigt eine rasterelektronenmikroskopische Aufnahme (siehe Abschnitt 2.6) einer hier verwendeten Sonde der Firma BUdGeTSENSORS ${ }^{\circledR}$, dessen Krümmungsradius mit $r<10 \mathrm{~nm}$ spezifiziert ist. Dabei ist zu beachten, dass der Elektronenstrahl des Mikroskops kohlenstoffbasier- 


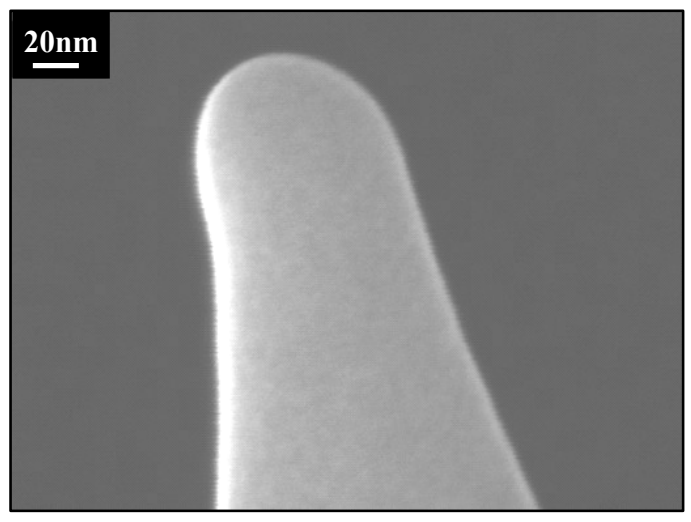

Abbildung 2.9: Rasterelektronenmikroskopische Aufnahme einer AFM-Spitze.

te Rückstände deponiert, die bei längerer Bestrahlung die Untersuchungen merklich beeinflussen. Eine Abschätzung des tatsächlichen Spitzenradius basierend auf mehreren Aufnahmen unterschiedlicher Spitzen ergab einen Wert von etwa $15 \mathrm{~nm}$. Systematische Abbildungsfehler wie die projektive Verbreiterung feinerer Strukturen durch die Spitzenfaltung und Kantenartefakte wurden in der Literatur ausgiebig diskutiert [Wie94, Ram98, Odi94].

\subsubsection{Statistische Analyse mittels der Autokorrelationsfunktion (ACF)}

Zur Auswertung der mittels AFM aufgezeichneten Oberflächentopographien in Hinblick auf Korrelationslängen wird häufig die Autokorrelationsfunktion herangezogen. Sie ist definiert als

$$
\mathrm{C}(\overrightarrow{\mathrm{r}})=\langle\mathrm{h}(\overrightarrow{\mathrm{x}}) \cdot \mathrm{h}(\overrightarrow{\mathrm{x}}+\overrightarrow{\mathrm{r}})\rangle_{\overrightarrow{\mathrm{x}}}
$$

wobei \langle\rangle$_{\vec{x}}$ hier für den Mittelwert über alle Koordinaten des betrachteten Raumes steht, denen ein Höhenwert h zugewiesen ist.

Für eine quantitative Auswertung ist die eindimensionale Autokorrelationsfunktion erforderlich. Sie lässt sich für AFM-Messungen, die auf Grund der Rasterung einen Datensatz diskreter Koordinaten liefern, berechnen aus

$$
C(x)=\frac{1}{M \cdot(N-x)} \sum_{m=1}^{M} \sum_{n=1}^{N-x} h_{n+x, m} h_{n, m},
$$

wobei N die Anzahl der Datenpunkte einer Rasterlinie und M die Anzahl der Rasterlinien ist, aus denen das entsprechende Bild zusammengesetzt ist. $\mathrm{x}$ gibt hierbei die laterale Koordinate gemessen in Bildpunkten an. 
Aus dieser Funktion lassen sich charakteristische Größen der Oberflächenstrukturen ablesen. Für kleine x ergibt sich das Maximum 0-ter Ordnung der Selbstkorrelation, wobei $\mathrm{C}(0)=\sigma^{2}$ dem Quadrat der mittleren quadratischen Rauigkeit (RMS-Rauigkeit) entspricht. Aus dem Abfall des 0-ten Maximums auf den e-ten Teil $\mathrm{C}\left(\mathrm{X}_{\mathrm{C}}\right)=\mathrm{C}(0) / \mathrm{e}$ lässt sich die Korrelationslänge $X_{C}$ bestimmen. Bei einer nahezu einheitlichen Größe räumlich separierter Hügelstrukturen gibt die Korrelationslänge Auskunft über die laterale Ausdehnung der Hügel. Besitzen diese Hügel außerdem einen nahezu einheitlichen Abstand zueinander, äußert sich dies in einem Maximum 1-ter Ordnung, wobei die Position $\mathrm{X}_{\mathrm{M}}$ des Maximums diesen Abstand angibt. Periodische Strukturen führen zu einer periodischen Autokorrelationsfunktion. Natürliche Oberflächen setzen sich aus unendlich vielen Wellenlängen unterschiedlicher Korrelationsstärken zusammen, die sich in der Autokorrelationsfunktion überlagern, daher lassen sich mit Hilfe dieser Funktion nicht alle Korrelationen direkt identifizieren.

\subsubsection{Berechnung der spektralen Leistungsdichte (PSD)}

Die spektrale Leistungsdichte wird durch Fourier-Transformation der Autokorrelationsfunktion erhalten

$$
\mathrm{C}(\overrightarrow{\mathrm{q}})=\mathrm{FT}(\mathrm{C}(\overrightarrow{\mathrm{r}}))
$$

und kann für den eindimensionalen Fall einer Mittelung über die horizontalen Rasterlinien mittels

$$
\mathrm{C}\left(\mathrm{q}_{\mathrm{x}}\right)=\frac{1}{\mathrm{NM}} \sum_{\mathrm{m}=0}^{\mathrm{M}}\left|\sum_{\mathrm{n}=0}^{\mathrm{N}} \mathrm{h}_{\mathrm{n}, \mathrm{m}} \exp \left(-\mathrm{iq}_{\mathrm{x}} \mathrm{nh}\right)\right|^{2}
$$

berechnet werden.

\subsection{Rasterelektronenmikroskopie (REM)}

Die Rasterelektronenmikroskopie erlaubt ähnlich wie die Rasterkraftmikroskopie eine Analyse der Oberflächenstruktur, wobei jede Methode für sich infolge unterschiedlicher Signalerzeugung spezifische Informationen liefert. Im REM wird ein fokussierter Elektronenstrahl über die Probe gerastert und die dadurch lokal erzeugten Sekundär- und Rückstreuelektronen detektiert. Die niederenergetischen Sekundärelektronen entstehen durch inelastische Streuung der Primärelektronen am Atomkern oder an den Elektronen der Atomhülle des Probenmaterials. Die höherenergetischen Rückstreuelektronen werden elastisch gestreut und erzeugen durch die starke Abhängigkeit des Rückstreukoeffizienten von der Ordnungszahl des Materials neben dem Topographie- auch einen Materialkontrast. Hierbei 


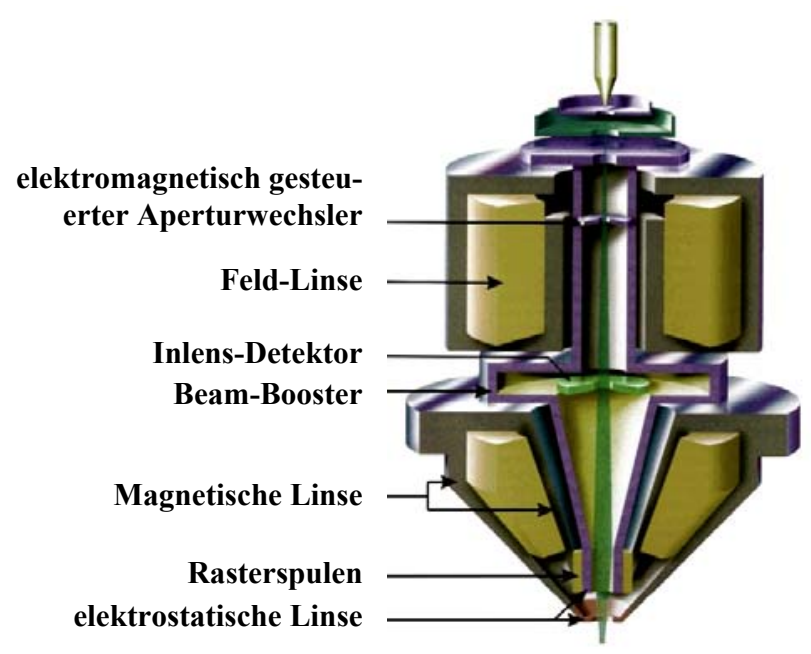

Abbildung 2.10: Schematischer Aufbau der Elektronenoptik des LEO Supra 35 Rasterelektronenmikroskops [Zei04].

entsteht ein zweidimensionales Bild der Oberfläche, das im Gegensatz zur quantitativen AFM-Analyse lediglich eine qualitative Höheninformation enthält. Spitzenfaltungseffekte werden hingegen vermieden und bei geeigneter Fokussierung werden laterale Auflösungen von etwa $5 \mathrm{~nm}$ erreicht. Bildfehler entstehen im Wesentlichen durch Abschattung in der Umgebung hoher Strukturen sowie Aufladungseffekte nicht oder nur schlecht leitender Materialien.

Die entsprechenden Untersuchungen wurden mit dem Rasterelektronenmikroskop LEO Supra 35 (Abbildung 2.10) durchgeführt, dass sich auf Grund der geometrischen Anordnung eines Inlens-Detektors direkt im Strahlengang und einer zusätzlichen Beschleunigung der Primärelektronen im so genannten Beam-Booster durch eine hohe Detektionseffizienz sowie geringere Störanfälligkeit bezüglich externer Felder auszeichnet.

\subsection{Energiedispersive Röntgenspektroskopie (EDX)}

Zur Materialanalyse mittels energiedispersiver Röntgenspektroskopie kann neben Sekundär- und Rückstreuelektronen in dem zuvor genannten REM (Abschnitt 2.6) außerdem die durch Wechselwirkung zwischen Elektronenstrahl und Probe erzeugte Röntgenstrahlung detektiert werden, wobei das Intensitätsverhältnis der elementspezifischen, so genannten charakteristischen Röntgenstrahlung eine quantitative Analyse der Probenzusammensetzung ermöglicht. Diese charakteristische Strahlung entsteht durch das Herausschlagen von Elektronen aus den inneren Schalen der Atome durch inelastische Stöße mit den Primärelektronen. Die freien Elektronenzustände werden durch Elektronen äußerer Schalen unter Emission der überschüssigen Energie in Form von Röntgenquanten besetzt. 
Mit Hilfe der Rastersteuerung des Elektronenstrahls ist nicht nur die integrale Analyse der gesamten Probe möglich, sondern eine örtliche Verteilung der Elementzusammensetzung bestimmbar. Die laterale Auflösung ist jedoch durch die Endringtiefe des Elektronenstrahls in das Material begrenzt. Die Primärelektronen werden beim Auftreffen auf das Material in der Probe gestreut, sodass die emittierte Röntgenstrahlung in einem birnenförmigen Raumvolumen mit einem Durchmesser von etwa $1 \mu \mathrm{m}$ entsteht. Bei der qualitativen Analyse der lateralen Elementverteilung von dünnen Schichten lässt sich eine deutlich höhere Auflösung erzielen, da das Anregungsvolumen oberflächlich nur eine Ausdehnung von etwa $20 \mathrm{~nm}$ besitzt. Eine quantitative Stöchiometriebestimmung sehr dünner Filme erfordert genaue Kenntnis der Schichtgeometrie und eine entsprechende Anpassung der Korrekturfaktoren für Ordnungszahl-, Absorptions- und Fluoreszenzkorrektur, da das Signal im Wesentlichen im Substrat erzeugt wird, und ist somit schwierig.

\subsection{Röntgen-Photoelektronenspektroskopie (XPS)}

Die Röntgen-Photoemissionsspektroskopie beruht auf der energieaufgelösten Detektion der infolge des Photoeffekts durch monochromatische Röntgenstrahlung aus inneren Schalen der untersuchten Materie ins Vakuum heraus gelösten Elektronen. Die kinetische Energie $E_{\text {kin }}$ der emittierten Elektronen hängt von der Energie der Elektronenzustände ab und ist somit für jedes Element charakteristisch. Sie entspricht der Differenz zwischen der Energie absorbierter Photonen $\mathrm{E}_{\text {Photon }}=\mathrm{h} v$ und der Summe aus Bindungsenergie $\mathrm{E}_{\mathrm{B}}$ und Austrittsarbeit $\mathrm{W}_{\mathrm{A}}$

$$
\mathrm{E}_{\mathrm{kin}}=\mathrm{h} v-\mathrm{E}_{\mathrm{B}}-\mathrm{W}_{\mathrm{A}}
$$

Mit Hilfe einer Datenbank lassen sich die XPS-Linien der jeweiligen Elemente identifizieren und prinzipiell anhand der gemessenen Intensität eine stöchiometrische Zusammensetzung der Probe ermitteln. Da das Signal durch diverse apparative Faktoren sowie der freien Weglänge der Elektronen und dem Photoionisationsquerschnitt abhängt, sind Absolutwerte bezüglich der Stöchiometrie ohne Verwendung entsprechender Referenzproben einem gewissen Fehler unterworfen. Angesichts der geringen freien Weglänge von Elektronen in Festkörpern von wenigen Nanometern und der Reduzierung der kinetischen Energie durch inelastische Streuung ist die XPS-Analyse eine oberflächensensitive Methode und erlaubt somit eine qualitative Untersuchung der chemischen Zusammensetzung von Oberflächen bzw. oberflächennaher Schichten.

Das verwendete XPS der Firma SPECS ist zwecks "in situ"-Oberflächencharakterisierung in die zur Herstellung der NiAg-Schichten (Abschnitt 2.1.2.3) genutzten Ultrahochvakuumanlage integriert und besitzt eine Röntgenquelle mit Al- $\left(\mathrm{E}\left(\mathrm{Al}-\mathrm{K}_{\alpha}\right)=1486.6 \mathrm{eV}\right)$ und $\mathrm{Mg}$-Kathode $\left(\mathrm{E}\left(\mathrm{Mg}-\mathrm{K}_{\alpha}\right)=1253.6 \mathrm{eV}\right)$. Da sich bei $1253.6 \mathrm{eV}$ Anregungsenergie die Signale von im Silber erzeugten Auger-Elektronen mit dem Ni2p Signal überlagern, wurde hier nur 
die Al-Kathode eingesetzt. Um so genannte extrinsische Elektronen, erzeugt durch inelastische Streuprozesse der Photoelektronen, bei der quantitativen Analyse der XPS-Linien zu eliminieren, erfolgte die Anwendung eines Shirley-Untergrundes [Shi72], der im Vergleich zum physikalisch zwar besser begründeten Tougaard-Untergrund [Tou85] eine genauere Peak-Anpassung erlaubte.

\subsection{Transmissionselektronenmikroskopie (TEM)}

Die Transmissionselektronenmikroskopie bietet die Möglichkeit einer hochaufgelösten Mikrostrukturanalyse, wobei im Gegensatz zur Rasterelektronenmikroskopie der Elektronenstrahl in Transmissionsgeometrie die Probe durchleuchtet. Der Signalkontrast entsteht hierbei durch die über Wechselwirkung mit den Atomen des Präparats gebeugten Elektronen des Primärstrahls ${ }^{11}$. Die Auflösung wird im Wesentlichen durch die Qualität der Elektronenlinsen sowie die Probendicke begrenzt und liegt im Hochauflösungsmodus (HRTEM) des hier verwendeten Philips CM200 des IV. Physikalischen Instituts der Universität Göttingen bei einer Beschleunigungsspannung von $200 \mathrm{kV}$ in der Größenordnung von $0.2 \mathrm{~nm}$. Um entsprechende elektronische Transparenz zu erzielen, sind Probendicken $<500 \mathrm{~nm}$ erforderlich. Durch den integrierten Röntgendetektor erlaubt das TEM zusätzlich eine ortsaufgelöste chemische Analyse mittels EDX, die auf Grund der geringen Ausbreitung des Anregungsvolumens in der Probe je nach Probendicke eine Genauigkeit von wenigen Nanometern erreicht. Die Messungen wurden in Zusammenarbeit mit PD. Dr. M. Seibt und. Dr. K. Thiel durchgeführt.

Die Herstellung geeignet dünner Proben bedingt ein spezielles Präparationsverfahren. Dies geschieht in Zusammenarbeit mit V. Radisch mit Hilfe eines auf $30 \mathrm{kV}$ beschleunigten, fokussierten Galliumionenstrahls (FIB) im FEI xT Nova Nanolab 200, das zur Abbildung und Positionierung außerdem eine konventionelle REM-Einheit beinhaltet. Der Galliumstrahl kann auf einen Durchmesser von etwa 40nm fokussiert und mittels Ablenksteuerung gezielt über die Probe geführt werden. Hierbei werden analog zum Sputterprozess über den Energieeintrag Atome aus der Probenoberfläche herausgeschlagen und ein definierter Materialabtrag erwirkt.

Im ersten Präparationsschritt wird das betreffende Gebiet durch chemische Gasphasenabscheidung (CVD) mit einer Platinschutzschicht versehen, um in dem später zu untersuchenden Probenbereich ionenstrahlinduzierte Modifikationen zu vermeiden. Das CVDVerfahren beruht auf der Abtrennung von Platin aus einer metallorganischen Verbindung, die gasförmig in die Kammer eingelassen wird, wobei die Spaltung unter Einwirkung des Elektronen- bzw. Ionenstrahls erfolgt. Anschließend werden Vertiefungen vor und hinter dem Platinstreifen erodiert und somit eine dünne Folie senkrecht zur Probenoberfläche aus-

\footnotetext{
${ }^{11}$ Eine detaillierte Beschreiung der Funktionsweise findet sich z.B. in [Ful02]
} 

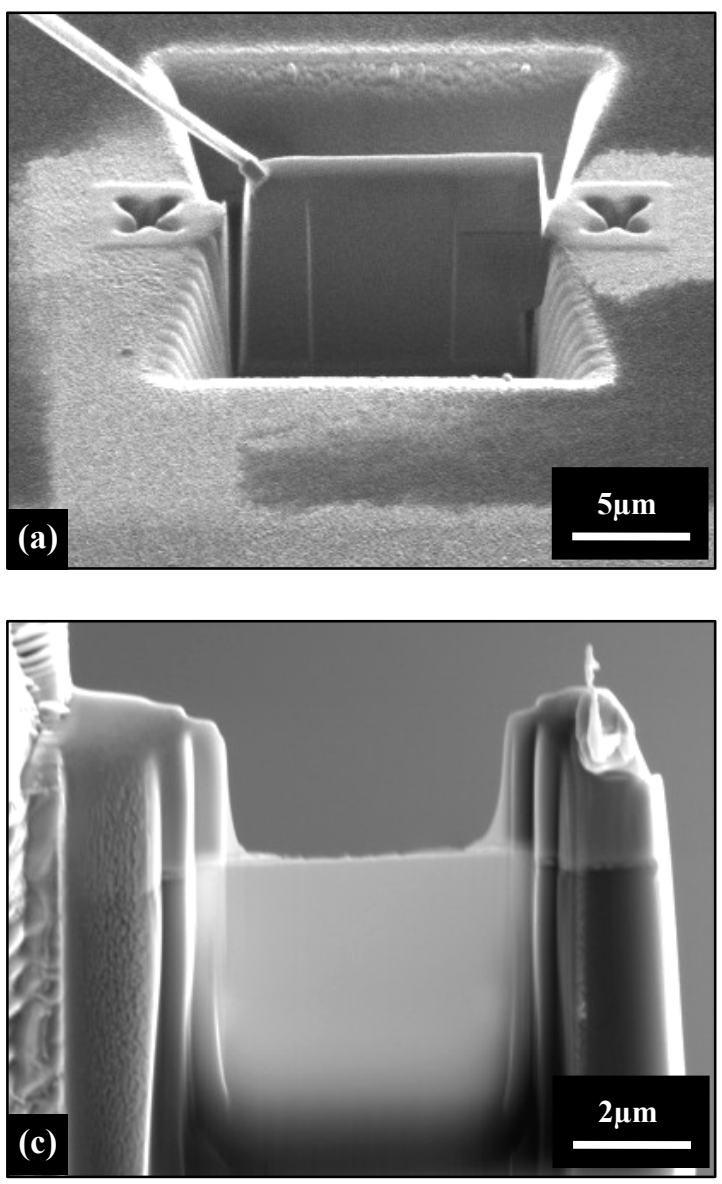

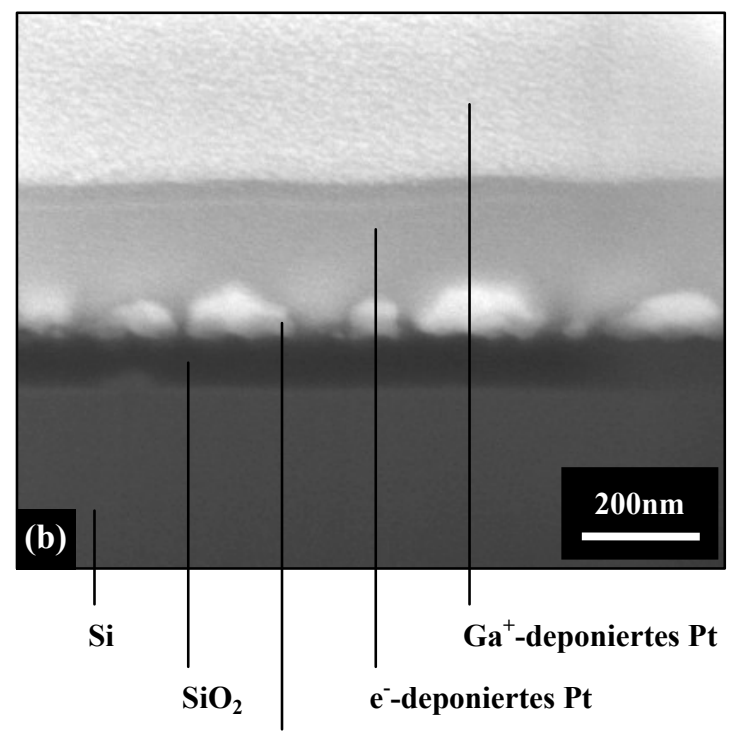

NiAg-Körner

Abbildung 2.11: REM-Aufnahmen der Präparation einer elektronentransparenten Lamelle mittels FIB: (a) senkrecht zur Probenoberfläche ausgeschnittene Folie mit $3 \mu \mathrm{m}$ dicker Pt-Schutzschicht an der Oberkante und seitlich befestigter Transfernadel, (b) Ausschnitt auf dieser Folie an der ursprünglichen Probenoberfläche, (c) entnommene und mittig weiter ausgedünnte, fertige Lamelle.

geschnitten (Abbildung 2.11(a)). Abbildung 2.11(b) zeigt den Querschnitt einer NiAgProbe auf thermisch oxidiertem $\mathrm{SiO}_{2}$ mit elektronen- und ionenstrahldeponierter PtSchutzschicht. Nach Befestigung der Transfernadel, was ebenfalls durch Platindeposition geschieht, wird der Dünnungsvorgang in der Mitte der Folie unter sukzessiver Reduzierung des Ionenstroms von beiden Seiten fortgesetzt. Abbildung 2.11(c) zeigt die entnommene Lamelle, die angesichts der abgetragenen Pt-Schicht in diesem Fall zu stark ausgedünnt wurde. 


\subsection{Magnetisierungsmessungen mittels magnetooptischen Kerreffekts (MOKE)}

Das Magnetisierungsverhalten auf rippelstrukturierten Substraten entnetzter Ni-Schichten sowie kodeponierter NiAg-Schichten wurde unter Ausnutzung des magnetooptischen Kerreffektes in Zusammenarbeit mit Dr. M. Djordjevic Kaufmann am IV. Physikalischen Institut der Universität Göttingen analysiert. Der Kerreffekt bezeichnet die Drehung der Polarisationsrichtung bei Reflektion polarisierten Lichts an einer magnetischen Probe in Abhängigkeit ihres Magnetisierungszustands. Hierbei ist der Kerrwinkel $\theta_{\mathrm{K}}$ direkt proportional zur Magnetisierung und liegt für ferromagnetische Übergangsmetalle in der Größenordnung von einigen Milligrad.

Der verwendete Messaufbau ist in Abbildung 2.12 dargestellt. Linear polarisiertes Laserlicht wird in einem $\lambda / 4$-Kompensator, bestehend aus einem $\lambda / 4$-Plättchen $\left(\theta=45^{\circ}\right)$ und einem Modulator, zirkular polarisiert und der horizontale Anteil der Lichtwelle mit einer Modulationsfrequenz von $50 \mathrm{kHz}$ alternierend um eine Phase von $\pm \pi / 2$ relativ zum vertikal polarisierten Anteil verschoben. Die Intensitätsmessung des reflektierten Strahls erfolgt nach Durchgang durch den Analysator anhand der Referenzmodulation mittels Lock-inTechnik.

Das Aufzeichnen von Magnetisierungskurven wird durch ein Helmholtzspulenpaar ermöglicht, welches in longitudinaler Geometrie des Messaufbaus ein variables externes Magnetfeld $\mathrm{H}_{\mathrm{ext}}$ erzeugt. Da aus der geringen Schichtdicke von 20nm bzw. 5nm ein stark eingeschränktes Signalrauschverhältnis resultiert, wurde jeweils über 20 Messungen gemittelt.

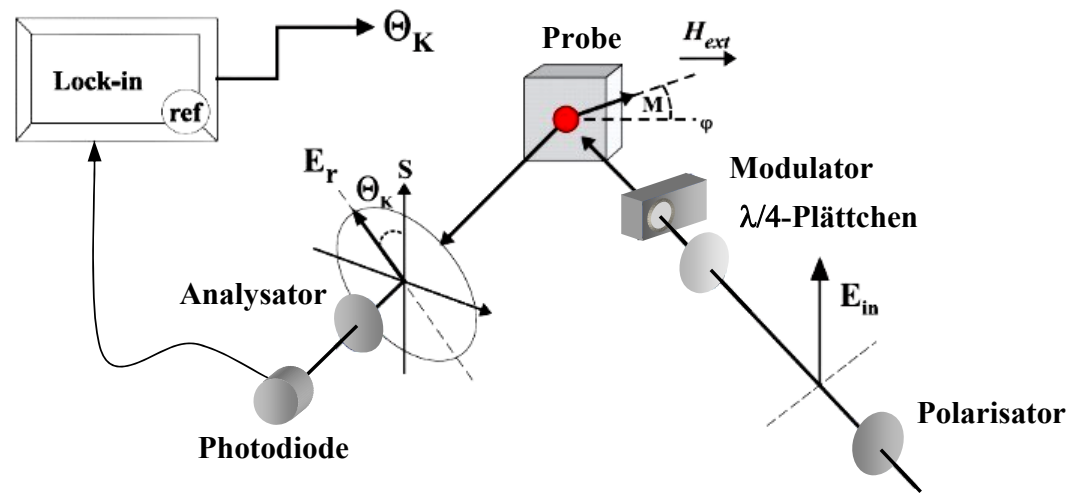

Abbildung 2.12: Aufbau zur Messung des magnetooptischen Kerreffekts. Abbildung nach [Kau06]. 


\subsection{Ionenbestrahlung am IONAS}

Zur Untersuchung der Modifikation von Grenzflächenrauigkeiten mittels Ionenbestrahlung wurden Ni/Ag-Doppellagen am Schwerionenimplanter IONAS des II. Physikalischen Instituts der Universität Göttingen bei Raumtemperatur mit unterschiedlichen Fluenzen von 300keV Xe $\mathrm{Xe}^{+}$-Ionen bestrahlt. Die Bestrahlung wurde von Dr. K. Zhang durchgeführt. Um thermisch induzierte Effekte durch ein globales Aufheizen der Proben während des Ioneneinschusses zu vermeiden, wurde ein wassergekühlter Kupferprobenhalter verwendet und der Ionenstrom mit $\mathrm{I} \leq 1 \mu \mathrm{A}$ bei einer bestrahlten Fläche von $1 \mathrm{~cm}^{2}$ entsprechend gering gehalten. Aufbau und technische Details des Beschleunigers sind in [Uhr85] näher erläutert.

Zwecks Ermittlung geeigneter Bestrahlungsparameter wurde das Monte-Carlo-Simulationsprogramm SRIM [Zie85] verwendet, das auf der Kalkulation von Stoßkaskaden sukzessiver binärer Kollisionen beruht und unter anderem die Berechnung von Ionenreichweiten, erzeugte Defektdichte und der Ionenimplantationsverteilung erlaubt. Ziel war es, in Hinblick auf Grenzflächenmodifikationen den Energieeintrag durch schwere, inerte Ionen an der Ni-Ag-Grenzfläche zu maximieren und dabei gleichzeitig strahlungsinduzierte Begleiteffekte wie z.B. Oberflächensputtern oder Ionenmischen an der Grenzfläche zum Substrat gering zu halten. Abbildung 2.13 zeigt die über $F_{D}=E_{d}\left(2 n_{v}+n_{d}\right)$ [Bo194] ermittelte deponierte Energiedichte pro Ion bei der Einstrahlung von $300 \mathrm{keV} \mathrm{Xe}{ }^{+}$-Ionen. $\mathrm{E}_{\mathrm{d}}$ bezeichnet hierbei die Gitterverlagerungsenergie, die mindestens erforderlich ist, ein Atom von seinem ursprünglichen Gitterplatz zu versetzen, und wurde mit $\mathrm{E}_{\mathrm{d}}=20 \mathrm{eV}$ angenommen. Die Parameter $n_{v}$ und $n_{d}$ bezeichnen die Anzahldichte erzeugter Leerstellen und der Ersetzungsstöße und wurden mittels SRIM berechnet. Das Maximum liegt etwas innerhalb der Nickelschicht, da zum Zeitpunkt der Bestrahlungsexperimente kein entsprechend stabiler Ionenfluss bei höherer Energie möglich war.
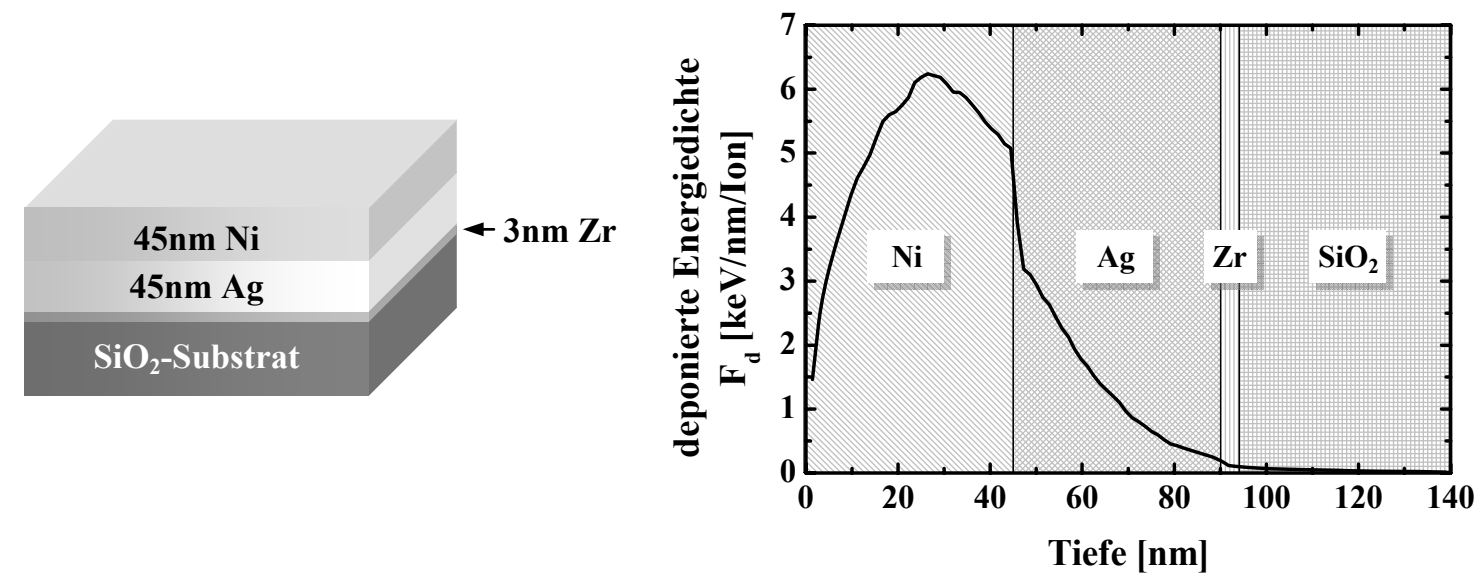

Abbildung 2.13: Verwendetes Schichtpaket (links) und Verteilung der deponierten Energiedichte bei Bestrahlung mit $300 \mathrm{keV} \mathrm{Xe}^{+}-$Ionen, berechnet mittels SRIM [Zie85] (rechts). 



\section{Kapitel}

\section{Glättung innerer Ni-Ag-Grenzflächen}

Das ursprüngliche Interesse an der Auswirkung der Ionenbestrahlung auf Festkörper gründete sich im Wesentlichen auf die Beobachtung von Strahlenschäden an Reaktorwänden und Abschirmungen im Zuge der Entwicklung von Kernreaktoren. Heutiges Interesse gilt unter anderem der gezielten Steuerung von strukturellen und magnetischen Eigenschaften dünner Schichten, der Herstellung von Zwangslegierungen fern vom thermodynamischen Gleichgewicht, sowie der Oberflächen- bzw. Grenzflächenstrukturierung. In diesem Kapitel soll zunächst eine kurze Zusammenfassung wesentlicher Effekte der Ionen-FestkörperWechselwirkung gegeben werden, bevor die Ergebnisse der Bestrahlung einer Ni-AgPhasengrenze bezüglich Rauigkeitsentwicklung sowie begleitender mikrostruktureller Veränderungen dargestellt werden.

\subsection{Grundlagen zur Ionen-Festkörper-Wechselwirkung}

Trifft ein Ion auf eine Festkörperoberfläche, findet auf Grund verschiedener Mechanismen ein Energieübertrag statt. Während bei hoher Ionenenergie von einigen $10 \mathrm{MeV}$ die elektronische Anregung durch Coulomb-Wechselwirkung mit den Elektronen der Festkörperatome überwiegt, kommt es im hier relevanten Energiebereich von bis zu einigen $100 \mathrm{keV}$ verstärkt zur nuklearen Anregung durch elastische Stöße mit den Atomkernen. Die Stöße können zu Verlagerungen von Atomen führen, wobei diese entweder auf einen anderen Gitterplatz oder einen Zwischengitterplatz verschoben und somit Punktdefekte wie Leerstellen und Zwischengitteratome erzeugt werden. Da das von dem einfallenden Ion getroffene Targetatom mit weiteren Atomen kollidiert und sich Stoßkaskaden ausbilden, bleibt der Wirkungsbereich des Ioneneinschlags nicht auf einen Gitterplatz beschränkt, sondern erstreckt sich über eine ganze Region entlang der Ionenbahn. Die sogenannte Kollisionsphase entwickelt sich rapide innerhalb etwa $10^{-13} \mathrm{~s}$ und lässt sich hinreichend durch sukzessive binäre Kollisionen beschreiben, wie es beispielsweise im Monte-Carlo-Simulationsprogramm SRIM [Zie85] umgesetzt wurde. Ist die Energie der Rückstoßatome unter den Schwellenwert $E_{d}$ für eine Atomverlagerung gefallen, finden keine weiteren Schäden durch 
elastische Stöße mehr statt. Die betroffenen Atome sind jedoch noch immer in Bewegung und ihre Energie reicht aus, um thermisch aktivierte Prozesse anzuregen. Zusätzlich wird durch die Rekombination von Leerstellen und Zwischengitteratomen lokal eine thermische Energie erzeugt, die meist einer Temperatur weit oberhalb des Schmelzpunktes entspricht, sodass stark ungeordnete Bereiche entstehen, in denen alle Atome simultan in Bewegung sind. Diese sogenannten Thermal-Spikes zeichnen sich durch hohe Drücke sowie Abkühlraten von $10^{12}-10^{15} \mathrm{~K} / \mathrm{s}$ aus und besitzen eine Lebensdauer von einigen Pikosekunden, bevor die Wärme vollständig an das umgebende Gitter abgegeben ist [Ave88]. Die mittlere kinetische Energie pro Atom innerhalb der Thermal-Spikes ist indes mit $\leq 1 \mathrm{eV}$ deutlich geringer als die Energie der Rückstoßatome während der Kollisionsphase, weshalb hier der atomare Transport sowie die Defekterzeugung maßgeblich von materialabhängigen thermochemischen Größen bestimmt werden [Ale93]. Die Wechselwirkungen können somit nicht mehr durch binäre Kollisionen beschrieben werden, sondern stellen vielmehr ein Vielkörperproblem dar und erfordern auf Grund ihrer Komplexität den Einsatz von Molekulardynamik-(MD)-Simulationen.

Der Energieübertrag durch die Abbremsung der Ionen kann neben Modifikationen der Oberflächenmorphologie infolge konkurrierender Aufrauungs- und Glättungsmechanismen über die Erhöhung der Mobilität von Oberflächenatomen beispielsweise durch angeregte Oberflächendiffusion bis hin zur Überwindung der Bindungsenergie und dem Sputtern der Targetatome auch unterschiedliche Effekte im Inneren des Festkörpers zur Folge haben. Hinsichtlich der Mechanismen der Oberflächenmodifikation durch Ionenbestrahlung soll auf das anschließende Kapitel verwiesen werden.

Im Volumen besteht bei der Bestrahlung mehrlagiger Schichtstrukturen die Möglichkeit des Ionenmischens. Dabei werden die unterschiedlichen Komponenten an den Grenzflächen entweder durch rein ballistische Effekte oder zusätzlich über Diffusion getrieben durch Gradienten des chemischen Potentials durchmischt. Somit können auch im Rahmen des Thermal-Spike-Modells, welches lokales Aufschmelzen bei hohen Abkühlraten impliziert, zum Teil homogene Zwangslegierungen von Komponenten mit positiver Mischungsenthalpie hergestellt werden, da viele entmischenden Systeme in flüssiger Phase mischbar sind [Ave86]. Krebs et al. [Kre95] fanden indes bei der Bestrahlung von Ag/FeDoppellagen, dass infolge der selbst in flüssiger Phase nicht mischbaren Komponenten ein offenbar chemisch getriebener Relaxationsmechanismus dem ballistischen Mischen entgegenwirkt, der vermutlich auf einen entmischenden Diffusionsprozess innerhalb der Thermal-Spikes zurückgeht.

Zudem kann die Bestrahlung unterschiedliche mechanische Spannungszustände in dünnen Schichten induzieren, die stark mit der mikrostrukturellen Entwicklung korreliert sind [May03]. Unter Ionenbestrahlung wird in der Regel ein Kornwachstum mit gleichförmiger Zunahme der mittleren Korngröße beobachtet. Die Kinetik des Kornwachstums und die Fluenzabhängigkeit der Korngröße L $(\Phi)$ sind meist vergleichbar mit dem Wachstumsver- 
halten bei isothermer Auslagerung, was eine identische treibende Kraft nahe legt, welche im Falle normalen Kornwachstums in der Minimierung der freien Energie durch Reduktion der Korngrenzenfläche besteht [Atk88]. Experimentell ermittelte Daten dünner einphasiger Schichten folgen dabei einem Potenzgesetz $\mathrm{L}^{\mathrm{n}}-\mathrm{L}_{0}^{\mathrm{n}} \propto \Phi$ mit Wachstumsexponenten $1.9 \leq$ $\mathrm{n} \leq 4.3$ [Ale91]. Mayr und Averback [May03b] konnten indes mit Hilfe von MDSimulationen an nanokristallinem NiAg den deutlichen Einfluss von viskosen Effekten innerhalb der Thermal-Spikes aufzeigen. Sie fanden, dass Kornwachstum, mechanische Spannungsentwicklung sowie Oberflächentopographie für Korngrößen unterhalb der typischen Ausdehnung von Thermal-Spikes von viskosem Fließen bestimmt werden und einer rapiden Änderung unterworfen sind, während die Kinetik der Effekte nach Überschreiten dieser Dimension deutlich zurückgeht.

\subsection{Rauigkeitsentwicklung}

Zur Untersuchung der Rauigkeitsentwicklung der inneren Phasengrenze im selbst in flüssiger Phase nicht mischbaren System Ni-Ag unter Ionenbestrahlung wurden mittels Elektronenstrahlverdampfens polykristalline $\mathrm{Ni}(45 \mathrm{~nm}) / \mathrm{Ag}(45 \mathrm{~nm}) / \mathrm{Zr}(4 \mathrm{~nm})$-Multilagen auf thermisch oxidiertes Silizium deponiert. Der Zirkonfilm dient hierbei lediglich als reaktive Lage zur Verbesserung der Adhäsion des Schichtpakets, da sich gezeigt hat, dass sich die nur schlecht haftenden Silberschichten vermutlich in Folge mechanischer Spannungen unter Ionenbestrahlung lokal vom Substrat ablösen. Die Substrattemperatur wurde während der Deposition auf 200K gehalten, um eine fein strukturierte Morphologie mit geringer Korngröße und signifikanter Grenzflächenrauigkeit zu erzielen. Anschließend wurden die Proben mit unterschiedlichen Fluenzen von $300 \mathrm{keV} \mathrm{Xe}$-Ionen unter senkrechtem Einfall
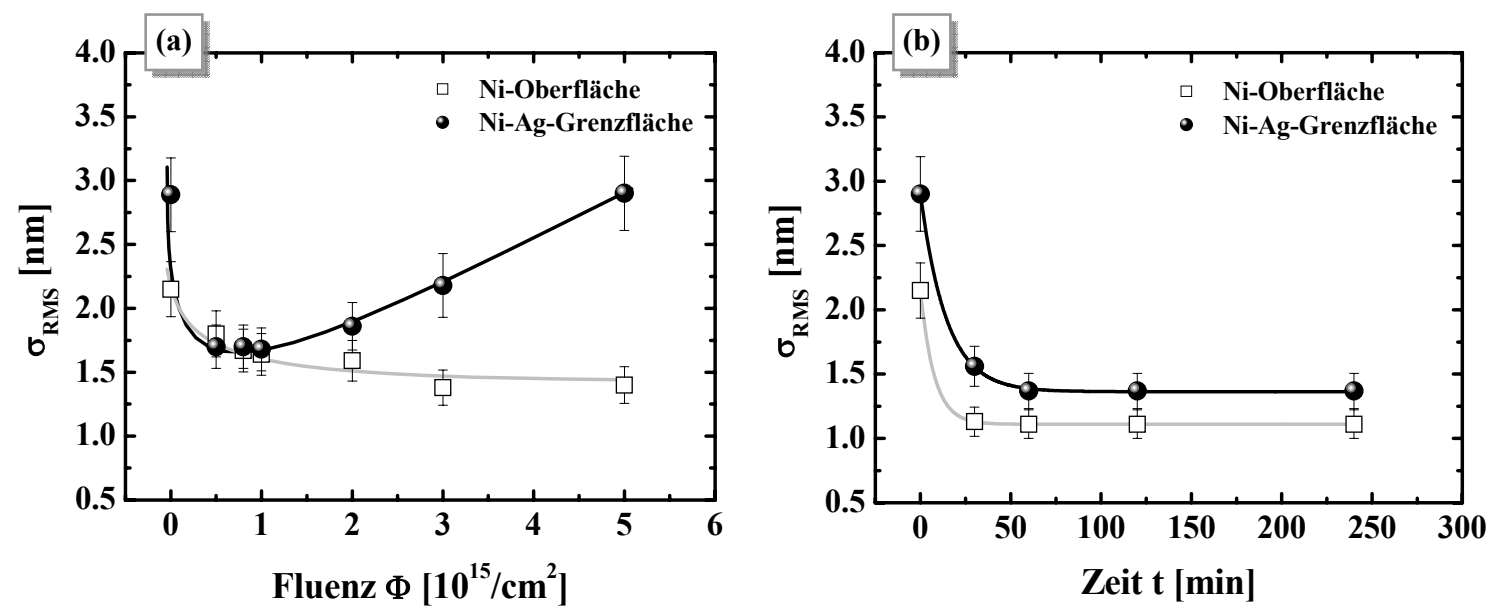

Abbildung 3.1: Rauigkeitsentwicklung der Oberfläche und der inneren Grenzfläche von Ni/Ag-Doppellagen (a) unter $300 \mathrm{keV} \mathrm{Xe}^{+}$-Bestrahlung sowie (b) unter thermischer Behandlung bei $500^{\circ} \mathrm{C}$. Die durchgezogenen Linien dienen lediglich dem Zwecke der Übersichtlichkeit. 
bezüglich der Schichtebene bestrahlt und eine Vergleichsprobe einer sukzessiven thermischen Behandlung bei $500^{\circ} \mathrm{C}$ im Ultrahochvakuum unterzogen.

Abbildung 3.1(a) zeigt die über XRR-Messungen (Anhang A) ermittelte Rauigkeit der Ni-Ag-Grenzfläche sowie der freien Nickeloberfläche bestrahlter Proben. Beide weisen für geringe Fluenzen $\Phi \leq 1 \cdot 10^{15} / \mathrm{cm}^{2}$ einen starken Glättungseffekt auf. Während die Oberflächenrauigkeit auf deutlich reduziertem Niveau oberhalb von $\Phi=1 \cdot 10^{15} / \mathrm{cm}^{2}$ jedoch praktisch konstant bleibt, wird für die Rauigkeit der inneren Grenzfläche ein nahezu linearer Anstieg mit der Fluenz beobachtet. Der Ausgangswert von $\sigma_{\mathrm{Ni}-\mathrm{Ag}, \mathrm{RMS}}(0)=2.9 \mathrm{~nm}$ vor der Bestrahlung wird nach Durchlaufen eines Minimums am Ende des hier betrachteten Fluenzbereichs $\Phi=5 \cdot 10^{15} / \mathrm{cm}^{2}$ schließlich überschritten.

Thermische Behandlung hat auf Grund der starken Glättungsreaktion zunächst einen ähnlichen Effekt auf die untersuchte Grenzflächenrauigkeit (Abbildung 3.1(b)). Die zeitliche Entwicklung unter Wärmeeinwirkung zeigt jedoch zwei signifikante Unterschiede zur Ionenbestrahlung. Zum einen stagniert die Rauigkeit der freien Oberfläche mit $\sigma_{\mathrm{Ni}, \mathrm{RMS}}=1.1 \mathrm{~nm}$ auf sichtlich geringerem Niveau, zum anderen zeigt die innere Grenzfläche auch für lange Auslagerungsdauer keinen anschließenden Aufrauungseffekt, sondern stagniert bei etwa $\sigma_{\mathrm{Ni}-\mathrm{Ag}, \mathrm{RMS}}=1.4 \mathrm{~nm}$.

\subsection{Kornwachstum}

Zur Ermittlung der Korngrößen wurden die Ag(111)- und Ni(111)-Signale der Röntgendiffraktogramme mittels Scherrer-Formel (siehe Abschnitt 2.3) ausgewertet. Dadurch lässt
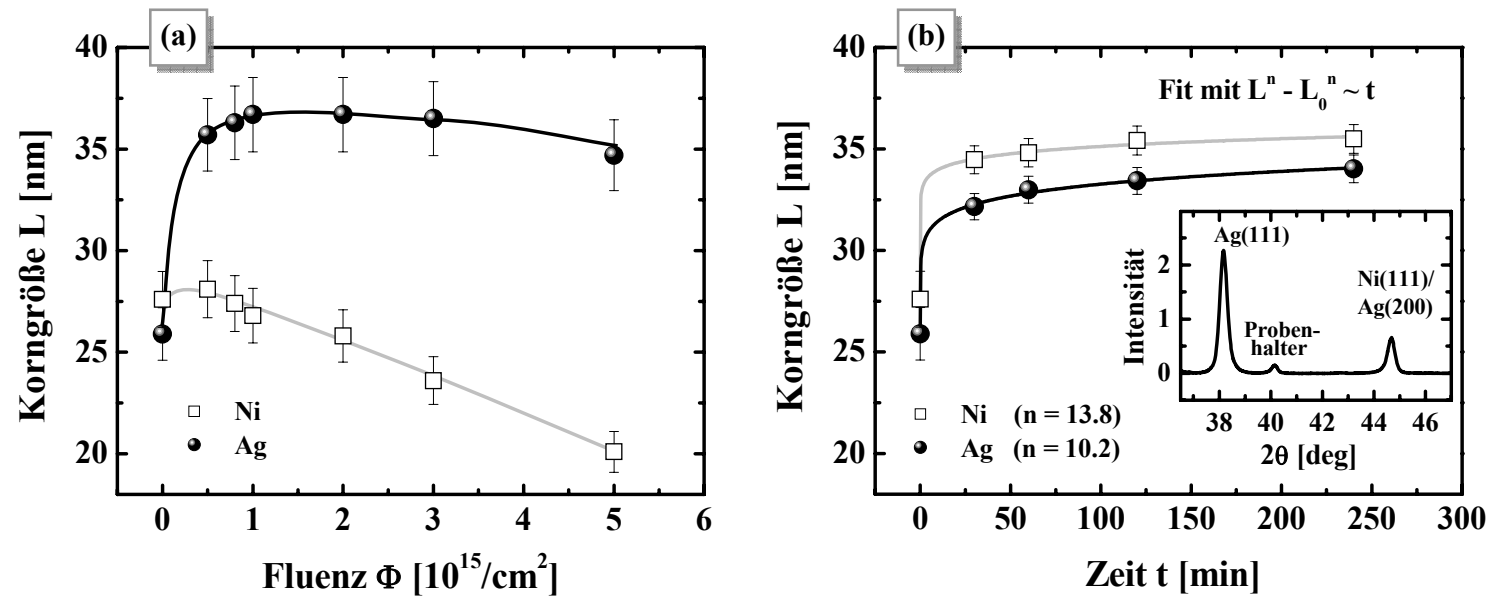

Abbildung 3.2: Entwicklung der Korngröße von Ni/Ag-Doppellagen (a) unter $300 \mathrm{keV} \mathrm{Xe}{ }^{+}$-Bestrahlung sowie (b) unter thermischer Behandlung bei $500^{\circ} \mathrm{C}$. Die durchgezogenen Linien dienen in (a) lediglich dem Zwecke der Übersichtlichkeit und stellen in (b) den Verlauf der jeweiligen Fit-Funktion dar. Der eingesetzte Graph zeigt das Röntgendiffraktogramm nach Schichtdeposition. 
sich die mittlere Ausdehnung kohärent streuender Bereiche senkrecht zur Schichtebene bestimmen, die im Allgemeinen mit der entsprechenden Ausdehnung der Körner identifiziert werden kann. Bei der Analyse des Ni(111)-Signals ist eine Überlagerung eines eventuell vorhandenen $\operatorname{Ag}(200)$-Signals nicht vollständig auszuschließen. Obwohl an den hier verwendeten Proben keine Texturmessungen durchgeführt wurden, zeigten Messungen an ähnlich präparierten Proben mit höherer Ni- bzw. Ag-Schichtdicke von jeweils 200nm eine starke Textur mit $<111>$-Wachstumsrichtung, wie es für fcc-Strukturen auf Grund dichtester Kugelpackung in der (111)-Ebene häufig beobachtet wird.

Das Röntgendiffraktogramm der hier verwendeten Proben direkt nach Deposition ist im eingesetzten Graph in Abbildung 3.2(b) dargestellt. Da neben den jeweiligen (111)Signalen keine höher indizierten Reflexe beobachtet werden konnten, ist auch hier von einer $<111>$-Wachstumsrichtung auszugehen, sodass das $\operatorname{Ag}(200)$-Signal vernachlässigbar klein sein dürfte. Auch nach Ionenbestrahlung bzw. nach thermischer Auslagerung sind keine weiteren Reflexe zu beobachten, was darauf schließen lässt, dass die Textur erhalten bleibt und keine strahlungsinduzierten Nichtgleichgewichtsphasen entstehen.

Die Modifikation der Grenzfläche wird von erheblichen mikrostrukturellen Veränderungen sowohl in der Nickel- als auch in der Silberschicht begleitet. Unter Ionenbestrahlung weist die Silberschicht eine anfänglich rapide Zunahme der Korngröße bis zu einer Fluenz von $\Phi=1 \cdot 10^{15} / \mathrm{cm}^{2}$ auf (Abbildung 3.2(a)). Für höhere Fluenzen ergibt die ScherrerAnalyse eine geringe Abnahme, wohingegen die entsprechenden Daten der Nickelkorngröße von Beginn an eine nahezu kontinuierliche Reduktion mit steigender Fluenz zeigen. Thermische Auslagerung hingegen resultiert in einem kontinuierlichen Kornwachstum (Abbildung 3.2(b)), wobei die mittlere Korngröße beider Lagen einem Potenzgesetz mit $\mathrm{L}^{\mathrm{n}}-\mathrm{L}_{0}^{\mathrm{n}} \propto \mathrm{t}$ und Wachstumsexponenten $\mathrm{n}_{\mathrm{Ni}}=14 \pm 2, \mathrm{n}_{\mathrm{Ag}}=10 \pm 1$ gehorcht.

\subsection{Diskussion}

\subsubsection{Kornwachstum}

Die Kinetik des Kornwachstums wird häufig über den Wachstumsexponenten n charakterisiert. Vergleichbar mit der Zeitabhängigkeit bei isothermer Auslagerung ergibt sich auch unter Ionenbestrahlung einphasiger dünner Schichten bei konstantem Ionenstrom durch Korngrenzenwanderung idealerweise eine Fluenzabhängigkeit der Korngröße $\mathrm{L}(\Phi)$ von $\mathrm{L}^{\mathrm{n}}-\mathrm{L}_{0}^{\mathrm{n}} \propto \Phi$ mit $\mathrm{n}=2$. Diese Beziehung lässt sich anhand der Ausbreitung einer Korngrenze durch die treibende Kraft zur Minimierung des Grenzflächen-zu-VolumenVerhältnisses bzw. der Grenzflächenkrümmung direkt über den Laplace-Druck ableiten [Atk88]. Defekte wie Verunreinigungen, Poren und Phasengrenzen behindern das Kornwachstum durch Einschränkung der Korngrenzenbeweglichkeit und führen zu einem Wachstumsexponenten von $\mathrm{n}>2$ [Gre73]. In den hier geführten Experimenten an $\mathrm{Ni} / \mathrm{Ag}$ - 
Doppellagen ist generell ein ähnliches Wachstumsverhalten zu erwarten. Die Kinetik wird jedoch dadurch eingeschränkt, dass sich die vertikale Ausdehnung der Körner senkrecht zur Schichtebene schnell der jeweiligen Filmdicke annähert, was in einer deutlich geringeren Wachstumsrate und entsprechend hohen Wachstumsexponenten resultiert. Dies äußert sich insbesondere im Kornwachstum der thermisch behandelten Probe, dessen Verlauf einem Potenzgesetz mit Wachstumsexponenten zwischen 10 und 14 gehorcht.

Im Falle der ionenbestrahlten Probe führt ein zusätzlicher Effekt zur scheinbaren Abnahme der Korngröße. Lokale Gitterverzerrungen infolge der Xe-Implantation sowie weitere strahlungsinduzierte Gitterdefekte reduzieren die Größe kohärent streuender Bereiche mit zunehmender Fluenz. Simulationen mittels SRIM [Zie85] zeigen eine maximale Xe-Konzentration an der Ni-Ag-Grenzfläche, wobei sich die Verteilung implantierter Ionen über beide Schichten erstreckt (Abbildung 3.3). Die defektbasierte Peak-Verbreiterung im Röntgendiffraktogramm täuscht folglich eine Reduktion der Korn-

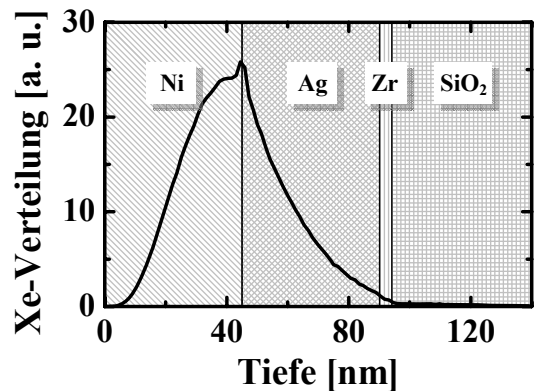

Abbildung 3.3: Verteilung der implantierten $\mathrm{Xe}^{+}$-Ionen nach Simulation mittels SRIM [Zie85]. größe vor.

\subsubsection{Rauigkeitsentwicklung}

Die Glättung der freien Nickeloberfläche unter Ionenbestrahlung zeigt qualitativ gute Übereinstimmung mit Untersuchungen bezüglich der Glättung von amorphen und polykristallinen Filmen, bei denen sich eine Dominanz viskosen Fließens innerhalb der Thermal-Spikes gegenüber strahlungsinduzierter Diffusion herausgestellt hat [May01, May03], daher ist es nahe liegend, dass derselbe Prozess ebenso die hier beobachtete Modifikation der Nickeloberfläche maßgeblich bestimmt. Im Gegensatz dazu wurden die atomaren Prozesse verantwortlich für die Glättung und Aufrauung der inneren Phasengrenze bislang nicht identifiziert.

Die Entwicklung der Grenzflächenrauigkeit verdeutlicht durch das auftretende Minimum das Wirken konkurrierender Glättungs- und Aufrauungsmechanismen und zeigt die Dominanz der Glättungsmechanismen bei geringen Fluenzen. Auf Grund der großen positiven Mischungsenthalpie $\Delta \mathrm{H}=23 \mathrm{~kJ} / \mathrm{mol}$ [Boe88] und der hohen Grenzflächenenergie des Systems besteht eine starke treibende Kraft zur Phasenseparation sowie zur Glättung durch Minimierung der Phasengrenzfläche. Dies wird besonders anhand der rapiden Rauigkeitsabnahme der thermisch behandelten Probe deutlich. Die Energieverteilung unter Ionenbestrahlung lässt sich hingegen klar von thermischen Auslagerungsexperimenten differenzieren, da hier nur lokale Stoßkaskaden erzeugt werden, während unter Wärmebehandlung 
ein thermisches Gleichgewicht besteht, sodass sich Atome praktisch durch den gesamten Film bewegen können.

Die anschließend beobachtete Aufrauung bei hohen Fluenzen lässt auf ballistische Effekte schließen und deutet den Einfluss der anfänglichen Kollisionsphase sowie der Bewegung strahlungsinduzierter Defekte nach der Thermal-Spike-Phase an. Gemäß dem Modell von Sigmund und Gras-Marti [Sig80, Sig81] findet ballistisches Ionenmischen immer statt, ist unabhängig von den chemischen Eigenschaften des Systems und hängt einzig von den kinematischen Eigenschaften des Materials ab. Experimentell konnte hingegen nachgewiesen werden, dass die Mischungsrate in Doppellagen hoher chemischer Affinität, sprich negativer Mischungsenthalpie, um ein Vielfaches höher ist, als rein ballistisches Mischen erwarten lässt [Joh85]. Selbst in flüssiger Phase nicht mischbare Systeme weisen demgegenüber eine wesentlich geringere Mischungsrate und zumindest bei niedrigen Fluenzen eine Schärfung der Grenzfläche auf [Kre95, Cre96]. Krebs et al. [Kre95] schlossen daher auf einen thermochemisch getriebenen Entmischungs- und Phasenseparationsprozess innerhalb der Thermal-Spike-Phase, welcher dem ballistischen Mischen entgegenwirkt. Die im Vergleich zum rein ballistischen Mischen höhere beobachtete Mischungsrate im System $\mathrm{Cu}-\mathrm{Fe}$, welches nur in flüssiger Phase mischbar ist, bekräftigt letztlich die Annahme eines flüssigkeitsähnlichen Verhaltens innerhalb der Thermal-Spikes sowie die Bedeutung des atomaren Transports während der Thermal-Spike-Phase [Cre03].

Der thermisch aktivierte und chemisch getriebene Entmischungsprozess ist somit weitestgehend begrenzt auf die Dimension der lokalen Thermal-Spikes, deren Ausdehnung hier in der Größenordnung von etwa 5nm [Neu99] liegt, sodass nur auf kurzer Distanz eine Phasenseparation stattfindet. Neubauer et al. [Neu96] fanden Punktdefekte in der Eisenschicht des zu Ni-Ag thermodynamisch vergleichbaren Systems Fe-Ag unter $\mathrm{Xe}^{+}$-Bestrahlung, die als Silberatome identifiziert werden konnten, und vermuteten langreichweitigen ballistischen Transport. Dieser Argumentation folgend werden einige wenige Nickelatome durch ballistisches Mischen von der zugehörigen Schicht isoliert und als Fremdatome tiefer in die Silberschicht eingebaut (und entsprechend Silberatome in die Nickelschicht), wo sie nicht an Phasenseparations- und Relaxationsprozessen der Grenzfläche teilnehmen können, während dem Transport auf kurzer Entfernung zur Grenzfläche die Entmischung innerhalb der Thermal-Spikes entgegenwirkt. Die für hohe Fluenzen resultierende Variation der Dichte und damit auch des Brechungsindizes $\mathrm{n}(\mathrm{z})$ senkrecht zur Schichtebene führt röntgenoptisch zu der in den XRR-Messungen beobachteten Aufweichung der Grenzfläche und somit zur Abnahme der gemessenen Reflektivität. Bei geringen Fluenzen überwiegt hingegen die Relaxation topographischer Unebenheiten der Phasengrenze getrieben durch Minimierung des Aufwands an Grenzflächenenergie, die zur Abnahme der gemessenen Rauigkeit führt.

Die zu den hier dargestellten Experimenten von Mayr durchgeführten MD-Simulationen bestätigen den rapiden Abfall der Grenzflächenrauigkeit bei niedrigen Fluenzen [Pet05]. Dabei zeigt die über Anlegen von Scherspannungen aus den Simulationen extrahierte 
Summe der Viskositäten $\eta_{1,2}=\eta_{\mathrm{Ag}}+\eta_{\mathrm{Ni}}=8.4 \mathrm{mPas}\left(\eta_{\mathrm{Ag}}=2.5 \mathrm{mPas}, \eta_{\mathrm{Ni}}=5.9 \mathrm{mPas}\right)$ der Nickel- und Silberschicht in flüssiger Phase gute Übereinstimmung mit der Summe der Viskositäten, die über die von Mullins aufgestellte Beziehung zur viskosen Relaxation von Oberflächenkorrugationen errechnet wurde. Mullins [Mu159] fand heraus, dass sinusförmige Oberflächenkorrugationen infolge viskosen Fließens exponentiell mit der Zeit abfallen, sodass sich für die Entwicklung der Rauigkeit letztlich

$$
\frac{\sigma(t)}{\sigma_{0}}=e^{-t / \tau} \quad \text { mit der Zeitkonstante } \quad \tau=\frac{2 \eta_{1,2}}{\gamma q}
$$

ergibt, wobei $\gamma$ die Grenzflächenenergie und q die Wellenzahl der Korrugationen bezeichnet. Mit einer Lebensdauer der Thermal-Spikes von etwa $t \approx 2.5 \mathrm{ps}$ liefert die in der Simulation beobachtete Rauigkeitsabnahme nach Auslösen einer Stoßkaskade über Gleichung 3.1 einen Wert von $\eta_{1,2}=\eta_{\mathrm{Ni}}+\eta_{\mathrm{Ag}}=9.1 \mathrm{mPas}$. Diese Übereinstimmung der Viskositäten bekräftigt die Annahme, dass auch für das Glätten der Grenzfläche viskoses Fließen den dominanten atomaren Mechanismus darstellt.

Das ausgeprägte Minimum in der fluenzabhängigen Rauigkeitsentwicklung zeigt, dass es prinzipiell möglich ist, auch innere Grenzflächen lokal oder flächendeckend zu glätten, ohne entsprechende Proben beispielsweise einer für viele elektronische Bauteile schädlichen Temperaturbehandlung zu unterziehen. Die von Erdélyi et al. [Erd04] beobachtete Schärfung der Grenzflächen infolge eines stark kompositionsabhängigen Diffusionskoeffizienten im mischbaren System Mo-V unter Wärmebehandlung legt nahe, dass eine Grenzflächenglättung durch Ionenbestrahlung bei ausreichend geringer Fluenz vermutlich auch in mischbaren Systemen unter dem Einfluss stark kompositionsabhängiger Viskositäten erzielt werden kann. 


\section{Kapitel}

\section{Rippelbildung}

Die Ionenbestrahlung ermöglicht neben spezifischer Veränderungen der inneren Struktur auch eine gezielte Oberflächenmodifikation von Festkörpern, wobei zwei Spezialfälle von besonderem technischen Interesse sind. Zum einen lassen sich durch eine Art Ionenpolieren extrem glatte Oberflächen herstellen [Spi89, Cha93, May01], zum anderen kann der Ionenstrahl abhängig von einer Vielzahl von Parametern eine hochgradig periodische Anordnung parallel ausgerichteter Wellenstrukturen, so genannte Rippel, als auch hexagonal geordnete Punktstrukturen [Fac99, Fro00] induzieren. In diesem Kapitel wird zunächst der theoretische Hintergrund der Rippelbildung durch Ionenbestrahlung kurz zusammengefasst und anschließend die Entwicklung und Charakterisierung geeignet strukturierter Substrate in Hinblick auf die Anwendung als Templates ${ }^{12}$ für die Strukturbildung deponierter Ni- und NiAg-Schichten dargestellt.

Die Rippelbildung durch Ionenbeschuss amorpher Festkörperoberflächen wurde erstmals 1962 von M. Navez et al. systematisch untersucht [Nav62] (Abbildung 4.1). Die hierbei entstehenden Wellenmuster erinnern stark an aeolische Sanddünen, bei denen die vorbeiströmende Luft durch lokale Verwirbelungen einen Abtrag von Sandkörnern bewirkt, die durch den Wind getrieben an anderer Stelle redeponiert werden. Sowohl die Rippelbildung durch Luftbewegung als auch durch Ionenbeschuss ist bislang nicht im Detail geklärt und Gegenstand aktueller Forschung [Kro05, Cha07]. So ähnlich sich die resultierenden Formationen auch sehen, so greift die Beschreibung ihrer Entstehung jedoch auf sehr unterschiedliche Ursachen zurück. Während das Sanddünenphänomen auf die strömungsphysikalische Wechselwirkung zwischen einem Fluid (Luft) und einem Granulat (Sand) zurückgeführt wird [Kro02], lassen die Ansätze zur Beschreibung der Modifikation von Festkörperoberflächen durch Ionenbeschuss die Redeposition des abgetragenen Materials weitestgehend außer Acht und behandeln als wesentliche Mechanismen das Aufbrechen von Bindungen und Ablösen von Oberflächenatomen durch den Energieeintrag des einfallenden Ions (Sputtererosion) sowie die thermisch oder durch ballistische Stoßprozesse angeregte

${ }^{12}$ Der Begriff „Templates“ (Englisch für Schablonen) wird als Bezeichnung für Vorlagen verwendet, die dazu dienen, bei einem Selbstorganisationsprozess eine bestimmte Strukturanordnung hervorzurufen. 

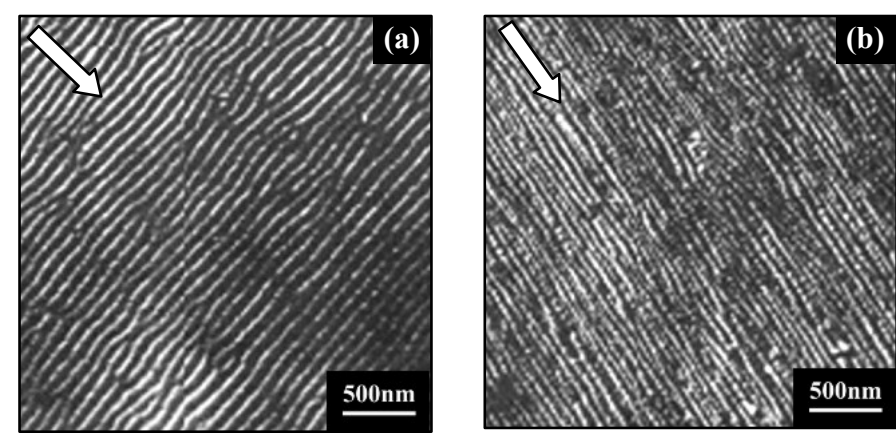

Abbildung 4.1: Topographie von mit ionisierter Luft bestrahlten Glassubstraten bei einer Energie von $4 \mathrm{keV}$ und unter einem Winkel von (a) $35^{\circ}$ bzw. (b) $80^{\circ}$ bezüglich der Oberflächennormalen [Nav62]. Die Richtung des einfallenden Ionenstrahls ist mit einem Pfeil angezeigt.

Oberflächendiffusion. In jüngster Zeit konnte indes die Relevanz weiterer Mechanismen nachgewiesen werden.

\subsection{Grundlagen zur Rippelbildung}

Bradley und Harper [Bra88] haben ein Kontinuumsmodell zur temporalen Evolution der Oberflächenmorphologie amorpher Festkörper entwickelt, das allgemein als theoretische Grundlage zur Klassifikation experimenteller Ergebnisse im Rahmen der Rippelbildung durch Ionenbestrahlung unter schrägem Einfall Verwendung findet. Die Basis stellt hierbei die Theorie von Sigmund [Sig73] dar. Ausgehend von einem Ionenstrahl konstanter Stromdichte wird dabei angenommen, dass die Sputterrate am Punkt P der betrachteten Oberfläche proportional zu der durch alle Einschläge in der Umgebung deponierten Energie an diesem Ort ist, wobei die deponierte Energie $F_{D}$ von der mittleren Wirkungstiefe a entlang und senkrecht zur Trajektorie des einfallenden Ions einer Gaußverteilung gehorcht (Abbildung 4.2):

$$
F_{D}(\widetilde{x}, \widetilde{y}, \widetilde{z})=\frac{E}{2 \pi^{2 / 3} \alpha \cdot \beta^{2}} \cdot \exp \left(\frac{(\widetilde{z}-a)^{2}}{2 \alpha^{2}}-\frac{\widetilde{x}^{2}+\widetilde{y}^{2}}{2 \beta^{2}}\right)
$$

(E: deponierte Gesamtenergie, a: mittlere Tiefe der Energiedeposition, $\alpha, \beta$ : Gaußbreiten der Verteilungsfunktion).

Anhand dieser Annahmen und einigen Rechenaufwands [Bra88] zur Bestimmung der lokalen Erosionsgeschwindigkeit, dessen Herleitung hier nicht weiter ausgeführt werden soll, ergibt sich eine krümmungsabhängige Sputterrate, die sich geometrisch durch Differenzierung der Fälle einer konvex und einer konkav gekrümmten Oberfläche erläutern lässt (Abbildung 4.2). Die deponierte Energie an einem Ort $\mathrm{O}$ negativer Krümmung, die durch 

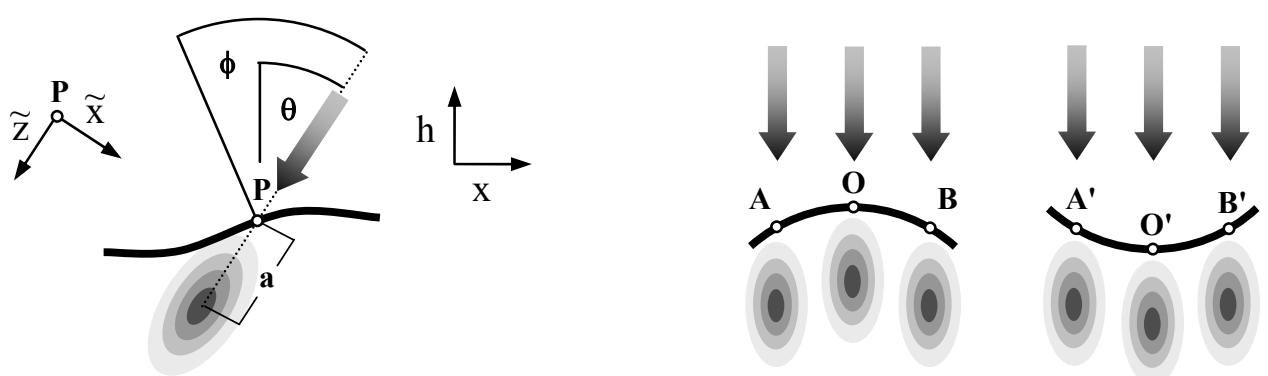

Abbildung 4.2: Darstellung der Energieverteilung beim Einschlag eines Ions (links) und Energieverteilung an Orten konvexer bzw. konkaver Krümmung unter Ioneneinfall (rechts).

Ioneneinschlag in eben diesem Punkt eingebracht wird, entspricht derer am Ort O' positiver Krümmung durch die dort auftreffenden Ionen. Da jedoch das Energiemaximum in A' bzw. B' auftreffender Ionen näher an O' liegt als das Maximum von in A bzw. B auftreffender Ionen an $\mathrm{O}$, wird effektiv mehr Energie an Orten positiver Krümmung deponiert und folglich eine höhere Erosionsgeschwindigkeit in Tälern der Oberfläche induziert. Neben der Krümmungsabhängigkeit ergibt sich weiterhin eine direkte Abhängigkeit der Abtragsrate vom lokalen Einfallswinkel $\phi$ des Ionenstrahls, die sich anhand analoger Überlegungen durch die oberflächennahe Energiedeposition bei flachem Einfallswinkel erschließt.

Unter der Bedingung, dass die Oberfläche bezüglich lokaler Gradienten nur leicht von einer ideal glatten Fläche abweicht, um die von Bradley und Harper angestellten Näherungen zu legitimieren, lässt sich eine lineare Differentialgleichung aufstellen, die die zeitliche Entwicklung der Oberfläche $\mathrm{h}(\mathrm{x}, \mathrm{y}, \mathrm{t})$ mit einem in der xh-Ebene unter dem globalen Winkel $\theta$ eintreffenden Ionenstrahl beschreibt:

$$
\frac{\partial \mathrm{h}}{\partial \mathrm{t}}=-v_{0}(\theta)+\frac{\partial v_{0}(\theta)}{\partial \theta} \frac{\partial \mathrm{h}}{\partial \mathrm{x}}+\frac{\mathrm{f} \cdot \mathrm{a}}{\mathrm{n}} \mathrm{Y}_{0}(\theta)\left[\Gamma_{\mathrm{x}}(\theta) \frac{\partial^{2} \mathrm{~h}}{\partial \mathrm{x}^{2}}+\Gamma_{\mathrm{y}}(\theta) \frac{\partial^{2} \mathrm{~h}}{\partial \mathrm{y}^{2}}\right]
$$

Hierbei bezeichnet $v_{0}(\theta)$ die mittlere Abtragsgeschwindigkeit, wobei $\partial v_{0} / \partial \theta$ als Rippelwanderungsgeschwindigkeit identifiziert werden kann, $f$ die Ionenstromdichte, $n$ die Anzahldichte der Targetatome, $\mathrm{Y}_{0}(\theta)$ den so genannten Sputter-Yield, der die mittlere Anzahl abgetragener Oberflächenatome pro Ioneneinschlag angibt, $\Gamma_{\mathrm{x}}(\theta)$ und $\Gamma_{\mathrm{y}}(\theta)$ zwei Parameter, die neben einer geometrischen Winkelabhängigkeit die Parameter a, $\alpha, \beta$ der Energieverteilung im Targetmaterial aus Gleichung 4.1 beinhalten. Die genauen Ausdrücke für $\Gamma_{\mathrm{x}}(\theta), \Gamma_{\mathrm{y}}(\theta)$ und $\mathrm{Y}_{0}(\theta)$ sind in Anhang B aufgeführt.

Ausgehend von einer regellosen, unebenen Oberfläche, dessen Fourier-Transformation das gesamte Frequenzspektrum umfasst, zeigen entsprechende Lösungen dieser Differentialgleichung, dass die Amplitude der Modulationen exponentiell anwächst, wobei Pertubati- 
onen kleiner Wellenlänge schneller divergieren. Um diese Diskrepanz zu experimentellen Untersuchungen zu beheben, die grundsätzlich eine endliche Wellenlänge der Rippelstrukturen offenbaren, wurde Gleichung 4.2 um einen Glättungsterm erweitert, welcher als krümmungsinduzierter Glättungsmechanismus der Aufrauung speziell im hochfrequenten Bereich entgegenwirkt:

$$
\frac{\partial \mathrm{h}}{\partial \mathrm{t}}=-v_{0}(\theta)+\frac{\partial v_{0}(\theta)}{\partial \theta} \frac{\partial \mathrm{h}}{\partial \mathrm{x}}+\frac{\mathrm{f} \cdot \mathrm{a}}{\mathrm{n}} \mathrm{Y}_{0}(\theta)\left[\Gamma_{\mathrm{x}}(\theta) \frac{\partial^{2} \mathrm{~h}}{\partial \mathrm{x}^{2}}+\Gamma_{\mathrm{y}}(\theta) \frac{\partial^{2} \mathrm{~h}}{\partial \mathrm{y}^{2}}\right]-\mathrm{K} \cdot \vec{\nabla}^{4} \mathrm{~h}
$$

In der ursprünglichen Form des Modells wurde thermisch aktivierte Oberflächendiffusion mit einer Relaxationsrate

$$
\mathrm{K} \propto \frac{1}{\mathrm{k}_{\mathrm{B}} \mathrm{T}} \mathrm{D}_{0} \exp \left(-\frac{\Delta \mathrm{E}}{\mathrm{k}_{\mathrm{B}} \mathrm{T}}\right)
$$

als Glättungsprozess berücksichtigt, wobei $\mathrm{D}_{0} \exp \left(-\Delta \mathrm{E} / \mathrm{k}_{\mathrm{B}} \mathrm{T}\right)$ den Selbstdiffusionskoeffizienten darstellt. Die konkurrierenden Prozesse der krümmungsinduzierten Glättung und Aufrauung durch Sputtererosion resultieren in einer Ausprägung periodischer Störungen mit einer Wellenlänge

$$
\lambda_{i}(\theta)=2 \pi \sqrt{\frac{2 n \cdot K}{f \cdot a \cdot Y_{0}(\theta) \cdot\left|\Gamma_{i}(\theta)\right|}}, \quad i=x, y .
$$

Infolge unterschiedlicher Winkelabhängigkeit der Parameter $\Gamma_{\mathrm{x}}$ und $\Gamma_{\mathrm{y}}$ dominieren bei kleinem Einfallswinkel $\theta$ Pertubationen mit einem Wellenvektor parallel zum projizierten Ionenstrahl. Wird ein kritischer Winkel überschritten, kommt es zur so genannten Rippelrotation und die Orientierung der Rippel wird um $90^{\circ}$ gedreht (Abbildung 4.1).

Das Bradley-Harper-Modell liefert qualitativ einige zutreffende Vorhersagen bezüglich Wellenlänge und Orientierung sich ausbildender Rippel und findet Anwendung auch im Bereich kristalliner Halbleiter, da diese meist durch Ioneneinschlag amorphisiert werden [Par65]. Es beinhaltet jedoch auch deutliche Einschränkungen. So findet das unbegrenzte, exponentielle Wachstum der Amplitude erfahrungsgemäß keine Rechtfertigung und bricht zwangsläufig mit der Voraussetzung einer nahezu glatten Oberfläche. Des Weiteren zeigen experimentelle Untersuchungen [Mac92, Dij01] z.B., dass eine Temperaturabhängigkeit der Wellenlänge nicht notwendigerweise auftritt und sich auch bei tiefen Temperaturen in Abwesenheit thermisch aktivierter Oberflächendiffusion eine Rippelstruktur mit definierter Wellenlänge ausbildet.

Neben Diffusionsprozessen wurde der Einfluss weiterer Glättungsmechanismen untersucht sowie ein im Mittel verschwindendes Rauschen $\eta(x, y, t)$ hinzugefügt, um der zufälli- 
gen Verteilung von Ioneneinschlägen Rechnung zu tragen [Cue95]. Moseler et al. [Mos05] konnten anhand von MD-Simulationen bei Energien $\mathrm{E} \leq 100 \mathrm{eV}$, bei denen kein Effekt lokalen Aufschmelzens nachgewiesen werden konnte, die Signifikanz eines ballistischen Glättungsprozesses zeigen. Dieser Prozess beruht auf der geometrischen Vorzugsrichtung der ballistischen Stoßkaskade bei nicht senkrechtem Ioneneinfall bezüglich der lokalen Steigung der Oberfläche, sodass die Impulskomponente parallel zur Oberfläche einen Partikelstrom entlang der Flanken der Hügel in die Täler erzeugt. Nach [Mos05] ergibt sich dadurch ein zusätzlicher Glättungsterm der Form $\partial \mathrm{h} / \partial \mathrm{t} \propto \vec{\nabla}^{2} \mathrm{~h}$.

Infolge der Ausbildung von Thermal-Spikes kann ebenso eine viskose Relaxation der Oberfläche auftreten. Basierend auf Betrachtungen von Orchard [Orc62] muss zwischen zwei Grenzfällen unterschieden werden, die kontinuierlich ineinander übergehen: das Fließen in einer dünnen Oberflächenschicht, das sich infolge der Krümmungsabhängigkeit wie die Oberflächendiffusion durch einen $\vec{\nabla}^{4} \mathrm{~h}$-Term über

$$
\frac{\partial \mathrm{h}}{\partial \mathrm{t}}=-\frac{\gamma \mathrm{a}_{\mathrm{z}}^{3}}{3 \eta} \vec{\nabla}^{4} \mathrm{~h}
$$

ausdrücken lässt [Oro97], wobei $\gamma$ die Oberflächenspannung, $a_{z}$ die Dicke der flüssigen Schicht und $\eta$ die Viskosität bezeichnet, und das Volumenfließen, bei dem die Dimension der flüssigen Schicht die typische laterale Strukturgröße der Oberfläche übersteigt. MDSimulationen amorpher Materialien unter senkrechtem Ioneneinfall von Vauth und Mayr [Vau07] belegten, dass im hier interessanten Energiebereich von einigen keV und Raumtemperatur viskoses Oberflächenfließen sowohl über Oberflächendiffusionsprozesse als auch (bis zu einer Strukturgröße in der Größenordnung von etwa $50 \mathrm{~nm}$ ) über ballistisches Glätten dominiert, sodass der entsprechende Ausdruck in Gleichung 4.3 durch $K=\gamma a_{z}^{3} / 3 \eta$ $\mathrm{zu}$ ersetzen ist.

Weiterhin haben numerische Rechnungen gezeigt, dass die Berücksichtigung von Termen höherer Ordnung zur Beschreibung des Sputterprozesses im Rahmen einer stochastischen, nichtlinearen Kontinuumsgleichung zu einer zeitlichen Separation der linearen und nichtlinearen Terme führt [Mak02]. Für kleine Zeitskalen (bzw. niedrige Fluenzen) können nichtlineare Terme vernachlässigt werden und das Modell liefert mit der Ausbildung periodischer Rippel und einem exponentiellen Amplitudenwachstum eine vergleichbare Oberflächenentwicklung wie das zuvor erläuterte Bradley-Harper-Modell. Auf längeren Zeitskalen dominieren die nichtlinearen Terme, die zur Stabilisierung der vorhandenen Rippelamplitude [Par99], zur Ausbildung einer veränderten Rippelstruktur oder auch zu einem dramatischen Anstieg kinetischer Aufrauung führen können.

Die theoretischen Modelle und Ansätze bilden ein solides Grundgerüst zur Beschreibung ionenstrahlinduzierter Strukturbildung auf Oberflächen und liefern zumindest ein 
qualitatives Verständnis der anfänglichen Morphologieentwicklung auf der Grundlage konkurrierender Glättungs- und Aufrauungsmechanismen verschiedener kinetischer Prozesse. Hinsichtlich der empfindlichen Abhängigkeit numerischer Lösungen komplexer nichtlinearer Differentialgleichungen von den Initialisierungsparametern sind Vorhersagen bezüglich der Spätstadien der Strukturbildung jedoch schwierig, weshalb wesentliche Erkenntnisse hinsichtlich der Auswirkung unterschiedlicher Bestrahlungsparameter bislang weitestgehend auf empirischen Beobachtungen beruhen. In Hinblick auf den Einsatz als Templates ist speziell die mangelnde Aussagekraft über den Ordnungsgrad und die maximal erreichbare Amplitude der Rippel zu erwähnen.

\subsection{Herstellung und Charakterisierung rippelstrukturierter Substrate}

Die Bildung periodischer Rippelstrukturen mit typischen Wellenlängen von etwa 10nm bis einigen Mikrometern ist Gegenstand vieler experimenteller Untersuchungen und weiterhin ein aktives Forschungsgebiet der Oberflächenphysik. Die resultierende Oberflächenmorphologie wird hierbei neben den Materialeigenschaften von einer Vielzahl extrinsischer Parameter beeinflusst:

- Einfallswinkel $\theta$ des Ionenstrahls bezüglich der Oberflächennormalen (hier verwendet: $20^{\circ} \leq \theta \leq 85^{\circ}$ )

- Ionenspezies (Argon, Krypton)

- Energie E $(0.6 \mathrm{keV} \leq \mathrm{E} \leq 5 \mathrm{keV})$

- Stromdichte $\mathrm{f}\left(1 \cdot 10^{-7} \mathrm{~A} / \mathrm{cm}^{2} \leq \mathrm{f} \leq 6 \cdot 10^{-5} \mathrm{~A} / \mathrm{cm}^{2}\right.$, soweit gemessen $)$

- Fluenz $\Phi\left(2 \cdot 10^{16} \mathrm{~cm}^{-2} \leq \Phi \leq 6.5 \cdot 10^{18} \mathrm{~cm}^{-2}\right.$, soweit gemessen $)$

- Substrattemperatur (Raumtemperatur sowie mit flüssigem Stickstoff gekühltes Substrat)

Ziberi et al. [Zib05] haben kürzlich über eine wohl definierte Rippelstruktur mit einer Wellenlänge von $60 \mathrm{~nm}$ und einer Tiefe von $5 \mathrm{~nm}$ berichtet, die durch Krypton-Bestrahlung von Silizium unter einem Winkel von $15^{\circ}$ erzeugt wurde. Bislang beobachtete Strukturen weisen jedoch bei Wellenlängen unter 100nm meist nur eine Amplitude von weniger als $5 \mathrm{~nm}$ auf und sind speziell auf Grund mangelnder Ordnung für die Anwendung als Templates im Rahmen dieser Untersuchungen größtenteils ungeeignet. In dieser Arbeit wurden Bestrahlungsexperimente dahingehend geführt, die folgenden Anforderungen zu erfüllen: 
- Eine kleine Wellenlänge ist erforderlich, da der atomare Transport während der Entnetzung dünner Filme in fester Phase begrenzt ist.

- Eine große Amplitude der Oberflächenkorrugationen in Verbindung mit einer kleinen Wellenlänge resultiert in deutlichen Krümmungsgradienten und gewährleistet eine starke Wechselwirkung zwischen Film und Rippelstruktur während der Entnetzung.

- Hoher Ordnungsgrad und geringe Defektdichte ermöglichen die Untersuchung des Entnetzungsprozesses auf den Templates unabhängig von dem Einfluss von Rippeldefekten auf den strukturbildenden Selbstorganisationsprozess.

Ein wesentlicher Aspekt dieser Arbeit bestand daher in der Entwicklung und Optimierung adäquater Substrate, wobei ein streng systematisches Vorgehen infolge der hohen Dimensionalität des Parameterraums hier nicht möglich war. Insgesamt wurden über 100 Substrate bestrahlt. Neben einer Vielzahl von Parameterkombinationen, bei denen keine Rippelbildung zu beobachten war, soll im Folgenden nur ein begrenzter Überblick über die unterschiedlichen beobachteten Formationen gegeben werden und in den theoretischen Hintergrund sowie aktuelle empirische Beschreibungen eingeordnet werden.

\subsubsection{Krypton-Bestrahlung}

Zunächst wurden Krypton-bestrahlte Proben bei Raumtemperatur mittels differentiell gepumpter Ionenquelle in der Analysekammer der UHV-Aufdampfanlage (Abschnitt 2.1.1.1) bei einem Druck von etwa $1 \cdot 10^{-8}$ mbar (bzw. einem Gasdruck von ca. $1 \cdot 10^{-4}$ mbar in der Ionenquelle) hergestellt. Hierfür wurde ein Edelstahlprobenhalter mit Drehvorrichtung konstruiert, dessen Winkelteilung eine auf zwei Grad genaue Justierung des Substrats zum Ionenstrahl ermöglicht. Der Ionenstrahl wurde mit Hilfe des eingebauten Faraday-Cups hinsichtlich maximalen Stroms fokussiert und unter Verwendung der Ablenkeinheit über eine etwa $1 \mathrm{~cm}$ x $1 \mathrm{~cm}$ große Fläche gerastert.

Als Substratmaterialien fanden sowohl Quarzglas, $\mathrm{Al}_{2} \mathrm{O}_{3}$, thermisch oxidiertes Silizium mit einer Oxidschichtdicke von etwa 500nm und einkristallines Silizium [Si(001)] Anwendung. Auf dem schon anfänglich sehr rauen $\mathrm{Al}_{2} \mathrm{O}_{3}$ konnte, bedingt durch den präferentiellen Abtrag in den Tälern und Abschattungseffekten, was im Wesentlichen zu einer verstärkten Ausprägung der ursprünglichen Struktur führte, keine langreichweitig geordnete Rippelstruktur erzeugt werden, wodurch sich diese Substrate als ungeeignet erwiesen. Die Verwendung von thermisch oxidiertem Silizium offenbarte bei entsprechender Ionenbestrahlung zur Herstellung ausreichend tiefer, periodischer Strukturen einen vollständigen Abtrag der Oxidschicht. Da wirklich brauchbare Ergebnisse letztendlich nur mit der Be- 
strahlung von reinen Siliziumsubstraten erzielt wurden, beschränkt sich die folgende Darstellung der Untersuchungen daher auf dieses Substratmaterial. Sämtliche Proben wurden mit dem projizierten Ionenstrahl parallel zur $<100>$-Richtung bestrahlt.

Abbildung 4.3 zeigt eine repräsentative Zusammenfassung der beobachteten Oberflächenentwicklung von Silizium bei Krypton-Bestrahlung mit einer Energie von $5 \mathrm{keV}$ bei unterschiedlichen Einfallswinkeln und einer Fluenz von $1 \cdot 10^{18} / \mathrm{cm}^{2}$ (dies entspricht einer Bestrahlungsdauer von etwa $48 \mathrm{~h}$ ). Für $70^{\circ}$ und $75^{\circ}$ wird außerdem zwischen zwei unterschiedlichen Fluenzen differenziert. Unter kleinen Einfallswinkeln (Abbildung 4.3(a)-(b)) ist keine präferentielle Ausrichtung der Oberflächenstrukturen zu erkennen und die Ober-

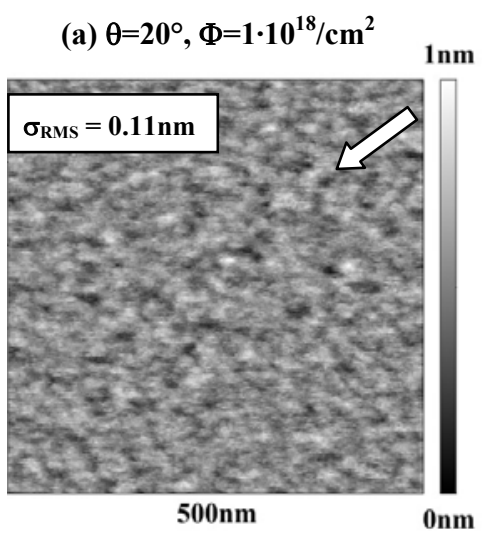

(d) $\theta=70^{\circ}, \Phi=6.5 \cdot 10^{18} / \mathrm{cm}^{2}$

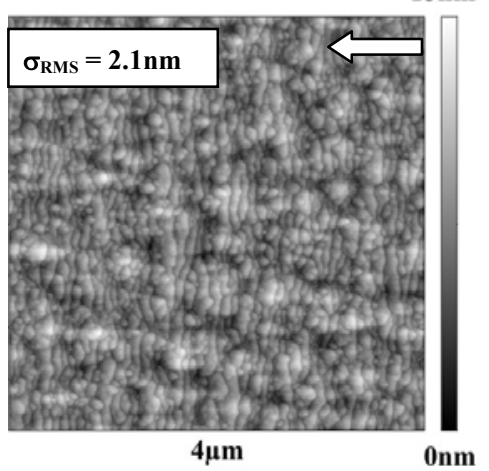

(b) $\theta=50^{\circ}, \Phi=1 \cdot 10^{18} / \mathrm{cm}^{2}$

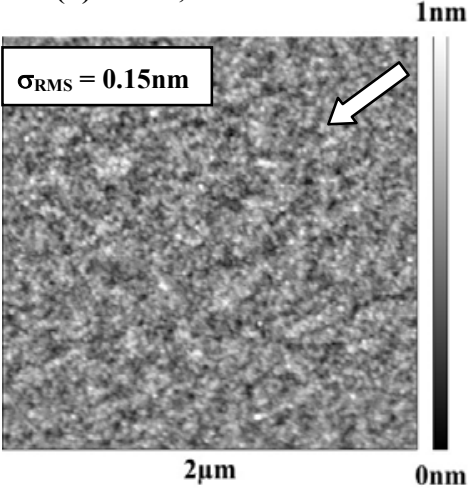

(e) $\theta=75^{\circ}, \Phi=2 \cdot 10^{17} / \mathrm{cm}^{2}$

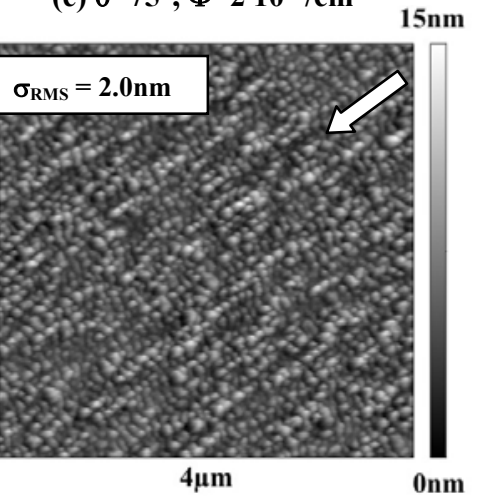

(c) $\theta=70^{\circ}, \Phi=1 \cdot 10^{18} / \mathrm{cm}^{2}$

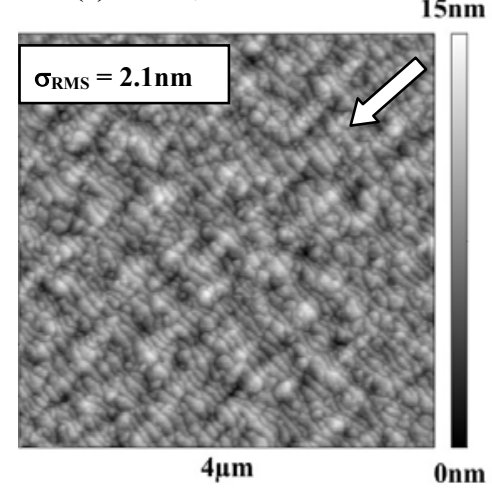

(f) $\theta=75^{\circ}, \Phi=1 \cdot 10^{18} / \mathrm{cm}^{2}$

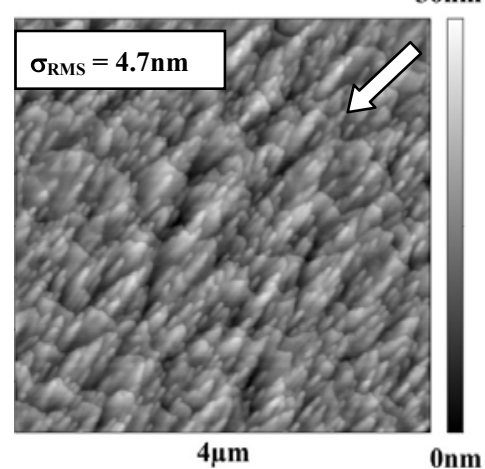

(g) Si(001) unbestrahlt

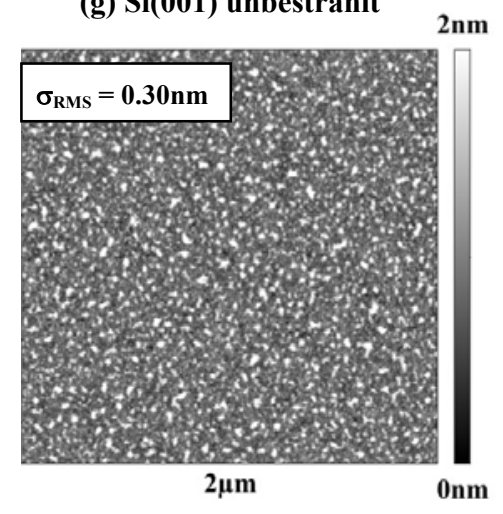

Abbildung 4.3: (a)-(f) AFM-Aufnahmen von mit $5 \mathrm{keV} \mathrm{Kr}^{+}$bestrahlten $\mathrm{Si}(001)$-Substraten bei unterschiedlichen Einfallswinkeln und Fluenzen sowie (g) unbestrahltes $\mathrm{Si}(001)$-Substrat. 
fläche ist mit einer RMS-Rauigkeit von $\sigma_{\mathrm{RMS}}=0.11 \mathrm{~nm}\left(20^{\circ}\right)$ bzw. $0.15 \mathrm{~nm}\left(50^{\circ}\right) \mathrm{im}$ Vergleich zum unbestrahlten Substrat (Abbildung $4.3(\mathrm{~g}), \sigma_{\mathrm{RMS}}=0.30 \mathrm{~nm}$ ) signifikant geglättet. $\mathrm{Zu}$ größeren Winkeln hin (Abbildung 4.3(c)) entwickelt sich eine stark gestörte periodische Struktur mit einer Wellenlänge von etwa $80 \mathrm{~nm}$ und Wellenvektor parallel zum projizierten Ionenstrahl. Senkrecht hierzu ist ebenfalls eine leichte Korrelation zu erkennen, die sich jedoch auf Grund mangelnder Ordnung nicht durch eine charakteristische Wellenlänge beschreiben lässt. Bei höheren Fluenzen (Abbildung 4.3(d)) bleibt diese Morphologie weitestgehend erhalten. Wird der Einfallswinkel weiter angehoben (Abbildung 4.3(e)), entsteht zunächst ein körniges Gefüge mit deutlicher Korrelation der Hügel entlang des projizierten Ionenstrahls und einer Wellenlänge von $95 \mathrm{~nm}$ sowie einer schwachen Korrelation senkrecht hierzu. Bei höheren Fluenzen (Abbildung 4.3(f)) kommt es zu einer Ausprägung parallel zum Ionenstrahl ausgerichteter, länglicher Strukturen und die ursprünglich dominierende Korrugation verschwindet.

\subsubsection{Orientierung der Rippel}

Das Verhalten der Rippelrotation wurde experimentell an diversen Materialien wie z.B. Graphit [Hab99], Quarzglas [Fla01] und kristallinen Metallschichten [Kar04] beobachtet. Die Einordnung in Vorhersagen der Kontinuumsmodelle wird indes durch meist nicht eindeutige Bestimmbarkeit des kritischen Winkels $\theta_{\mathrm{C}}$ erschwert. Um einen Vergleich mit dem Bradley-Harper-Modell anzustellen, wurden die Parameter a, $\alpha, \beta$ der Energieverteilung von $5 \mathrm{keV} \mathrm{Kr}^{+}$-Ionen mit Hilfe des Simulationsprogramms SRIM [Zie85] errechnet und die resultierende Wellenlänge über Gleichung 4.5 sowie der in Anhang B aufgeführten Ausdrücke bestimmt. Nach dem Bradley-Harper-Modell wird die Orientierung der Rippel allein durch die Richtung des Ionenstrahls beeinflusst, wobei in Bezug auf den Einfallswinkel jene Pertubationen dominieren, die den geringeren Wert $\left|\Gamma_{\mathrm{i}}(\theta)\right|$ und folglich die größere Wellenlänge aufweisen. Abbildung 4.4 zeigt die erwartete Winkelabhängigkeit der normierten Wellenlänge mit einem kritischen Winkel von $\theta_{\mathrm{C}}=67^{\circ}$. Dieser theoretisch begründete kritische Winkel der Rippelrotation liegt etwas unterhalb des tatsächlich beobachteten Wertes $70^{\circ}<\theta_{\mathrm{C}}<75^{\circ}$. Die hier durchgeführten Experimente offenbaren, dass eine Rippelstruktur mit einer Ausrichtung entlang des Ionenstrahls allgemein weniger regelmäßig angeordnet und bei Bestrahlung ab einem Winkel von etwa $70^{\circ} \mathrm{zu}$ beobachten ist. Die Dominanz gegenüber senkrechter Ausrichtung zeigt sich indes erst unter $75^{\circ}$-Bestrahlung bei hohen Fluenzen. Eine Rippelrotation mit voranschreitender Bestrahlungsdauer wurde auch in Experimenten an Silizium von Brown und Erlebacher [Bro05] beobachtet. Dieses Verhalten ist analytisch bislang nicht belegt. Mögliche Ursache ist die Sättigung der dominierenden Rippelwachstumsmode auf Grund nichtlinearer Effekte und damit eine Ausprägung der sich langsamer entwickelnden Mode [Bro05]. 


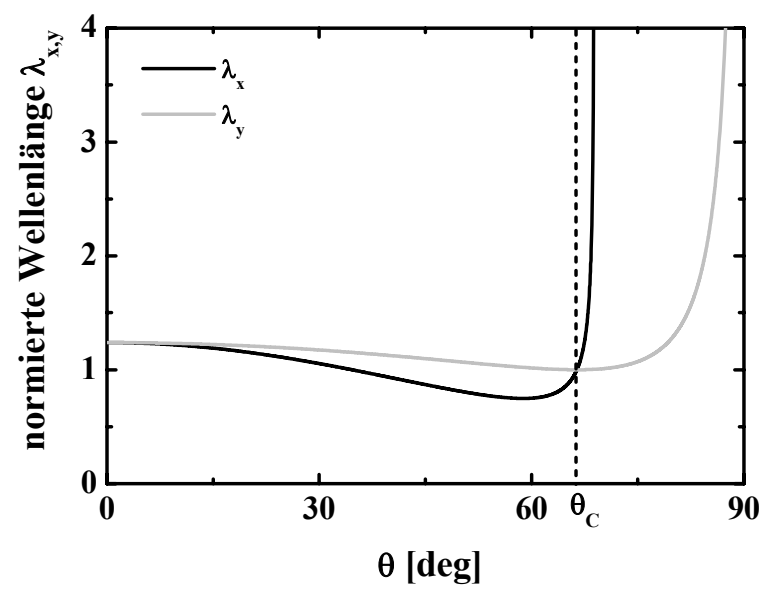

Abbildung 4.4: Winkelabhängigkeit der auf $\lambda\left(\theta_{\mathrm{C}}=67^{\circ}\right)$ normierten Wellenlänge unter $5 \mathrm{keV} \mathrm{Kr}^{+}$Ionenbestrahlung nach dem Bradley-Harper-Modell.

Im Gegensatz zur Fluenzabhängigkeit haben Rusponi et al. [Rus98] bei Kupfer eine Temperaturabhängigkeit der Rippelrotation nachgewiesen und führen dies auf zwei Regime zurück. Bei niedrigen Temperaturen beherrscht im Wesentlichen Erosion die Oberflächenmorphologie, wohingegen es bei höheren Temperaturen und der damit angeregten Diffusion zu einer Drehung der Pertubationen kommt. Hierbei spielt jedoch die kristalline Struktur des Kupfers und die Richtung des Ionenstrahls bezüglich kristallographischer Achsen eine entscheidende Rolle. Silizium wird durch Ioneneinschlag im keV-Energiebereich schon bei Fluenzen von $10^{14} / \mathrm{cm}^{2}$ oberflächlich amorphisiert [Rev78, Chi03], also deutlich bevor sich eine Rippelstruktur ausbildet. Der Einfluss von Anisotropieeffekten der Oberflächendiffusion und Ehrlich-Schwoebel-Barrieren wird somit im Allgemeinen ausgeschlossen, wodurch ein Vergleich mit kristallinen metallischen Oberflächen infolge unterschiedlicher Mechanismen nur eingeschränkt möglich ist.

\subsubsection{Rauigkeit}

Abbildung 4.5 zeigt den Verlauf der RMS-Rauigkeit in Abhängigkeit vom Bestrahlungswinkel. Neben einer deutlichen Glättung der Oberfläche bei kleinen Winkeln ist ein rapider Anstieg der Rauigkeit bei flachem Ioneneinfall zu erkennen. Die Evolution der Oberflächenmorphologie wird durch konkurrierende Glättungs- und Aufrauungsmechanismen bestimmt, wobei für Einfallswinkel im Bereich um $45^{\circ}$ im Widerspruch zum Bradley-HarperModell keine Rippelbildung beobachtet wurde [Fro04, Zib05]. Für eine Aufrauung ist im Wesentlichen der durch stochastischen Ioneneinschlag induzierte Sputterprozess verantwortlich, dessen Sputter-Yield von lokalen Oberflächenkrümmungen und -gradienten abhängt. Die bei flacherem Ioneneinfall oberflächennähere Energiedeposition resultiert in einem mit zunehmendem Winkel $\theta$ ansteigenden Sputter-Yield Y( $\theta)$. Sigmund [Sig69] hat 


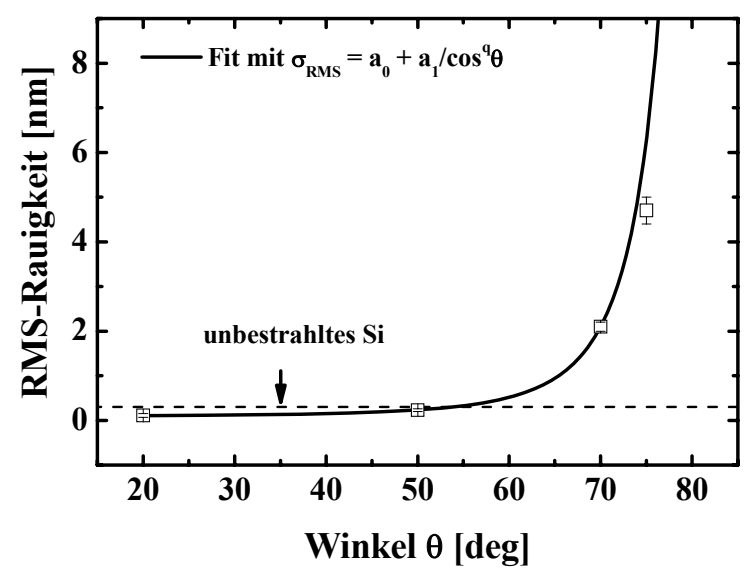

Abbildung 4.5: Winkelabhängigkeit der RMS-Rauigkeit von mit $\mathrm{Kr}^{+}$-Ionen bestrahltem Si bei einer Energie von $5 \mathrm{keV}$ und einer Fluenz von $1 \cdot 10^{18} / \mathrm{cm}^{2}$.

hierfür die Beziehung $\mathrm{Y}(\theta) \propto 1 / \cos \theta$ abgeleitet. Experimentelle Untersuchungen zeigen jedoch meist einen Zusammenhang der Form $\mathrm{Y}(\theta) \propto 1 / \cos ^{\mathrm{q}} \theta$ mit $\mathrm{q}>1$ für nicht zu streifenden Einfall. Bei großen Winkeln reicht die transversale Komponente des Energieübertrags nicht aus, um eine Kollisionskaskade zu initiieren. Die Ionen werden durch repulsive Wechselwirkung an der Oberfläche reflektiert, sodass der Sputter-Yield um etwa $70^{\circ}$ ein Maximum erreicht [Oec73] und für $\theta$ nahe $90^{\circ}$ verschwindet. Ein ähnliches Verhalten äußert sich hier in dem Verlauf der RMS-Rauigkeit (Abbildung 4.5), das sich trotz des Einflusses von Glättungsmechanismen auf Grund der starken Winkelabhängigkeit des Sputter-Yields bis einschließlich $70^{\circ}$ gut durch ein $\sigma_{\mathrm{RMS}}(\theta)=\mathrm{a}_{1}+\mathrm{a}_{2} / \cos ^{\mathrm{q}} \theta$-Gesetz mit $\mathrm{q} \approx 4$ beschreiben lässt, wobei der nach unten abweichende Wert für $\theta=75^{\circ}$ auf die zuvor erläuterte Abnahme des Energieeintrags zurückzuführen ist. Da nicht stets von einer glatten Oberfläche ausgegangen werden kann und die Aufrauung im Zuge des Bestrahlungsvorgangs zur Modifikation des lokalen Einfallswinkels führt sowie nichtlineare Terme bei hohen Fluenzen eine Sättigung der Aufrauungsinstabilität vermitteln können, ist allerdings eine direkte Korrelation zwischen dem Verhalten des auf glatte Flächen bezogenen SputterYields und der nach gewisser Erosionszeit beobachteten RMS-Rauigkeit nicht notwendigerweise gegeben.

In Bezug auf die Oberflächenglättung nahe senkrechtem Ioneneinfall sei auf die von Vauth und Mayr [Vau07] gezeigte Dominanz viskosen Oberflächenfließens verwiesen. Dieser Relaxationsprozess überwiegt unter $5 \mathrm{keV}$-Krypton-Bestrahlung vermutlich auch im hier untersuchten Winkelbereich zwischen $20^{\circ} \leq \theta \leq 50^{\circ}$ den Aufrauungseffekt der Sputtererosion und unterdrückt somit die Rippelbildung. Bei $\theta=70^{\circ}$ entwickelt sich zwar eine kurzwellige Rippelstruktur mit etwa 80nm Wellenlänge und einer Rippeltiefe von etwa $3 \mathrm{~nm}$, keines der untersuchten Substrate erfüllt jedoch die zuvor genannten Kriterien speziell hinsichtlich erforderlicher Ordnung. 


\subsubsection{Argon-Bestrahlung}

Neben dem Einfallswinkel und der Fluenz wird unter Verwendung von Argon-Sputtergas nun ebenfalls die Ionenspezies sowie die Ionenenergie und Substrattemperatur variiert. Entsprechende Bestrahlungen wurden zum einen in der in Abschnitt 2.1.2.3 beschriebenen UHV-Anlage per differentiell gepumpter Ionenquelle mit Rastereinheit bei einem Druck von $\mathrm{p} \approx 5 \cdot 10^{-7}$ mbar, zum anderen in der Ionenmühle GATAN 600 TMP Duo Ion Mill des IV. Physikalischen Instituts der Universität Göttingen durchgeführt. Die Ionenmühle bietet deutlich höhere Stromdichten (bis zu $10 \mathrm{~mA} / \mathrm{cm}^{2}$ ) als herkömmliche Ionenquellen und ermöglicht eine Kühlung des Substrats mittels flüssigen Stickstoffs. Sie besitzt hingegen keine Vorrichtung zum Rastern oder zur Defokussierung des Strahls, wodurch eine eventuelle Rippelbildung nicht homogen auf der gesamten Probe sondern nur lokal induziert werden kann. Aus Ermangelung eines integrierten Faraday-Cups erfolgt eine relative Fluenzangabe in Form der Bestrahlungsdauer.

Obwohl im Falle eines gerasterten Strahls als auch bei einem festen, defokussierten Strahl üblicherweise von einer homogenen Bestrahlung ausgegangen wird, können lokale und temporale Variationen des stochastischen Sputterprozesses sowie Störungen eines einheitlichen Ionenflusses Auswirkungen auf die resultierende Strukturbildung haben. Abbildung 4.6 zeigt die unterschiedliche Oberflächenmorphologie zweier unter vergleichbaren Bedingungen $\left(\theta=75^{\circ}, \mathrm{E}=0.8 \mathrm{keV}\right.$, Bestrahlungsdauer $\mathrm{t}=48 \mathrm{~h}$ und Raumtemperatur) in der UHV-Anlage mit festem, defokussierten bzw. mit gerastertem Ionenstrahl bestrahlten Substrate. Beide weisen eine Rippelstruktur ähnlicher Charakteristika mit einer Wellenlänge von etwa $50 \mathrm{~nm}$ und $2.5 \mathrm{~nm}$ mittlerer Rippeltiefe auf. Unter Verwendung der zeilenweisen Abtastung wird jedoch trotz höherer RMS-Rauigkeit eine deutlich höhere Konformität betreffend paralleler Anordnung und Amplitude benachbarter Wellenformationen erzielt. Chini et al. [Chi02] haben diesbezüglich eine gegensätzliche Energieabhängigkeit

(a) fester Strahl

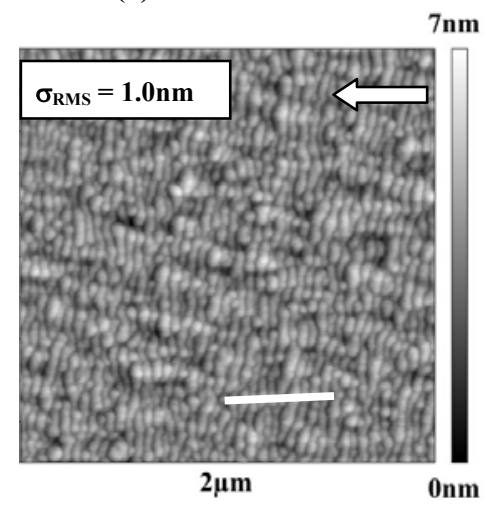

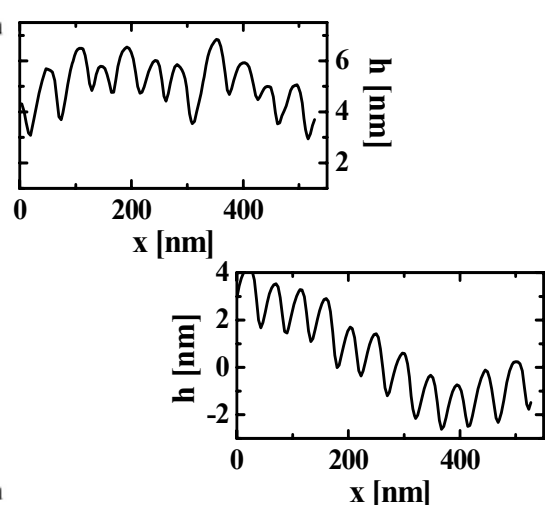

(b) gerasterter Strahl

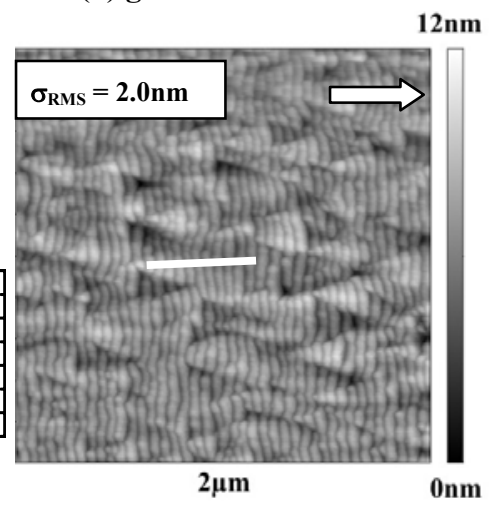

Abbildung 4.6: AFM-Aufnahmen eines unter vergleichbaren Bedingungen (a) mit festem, defokussierten Ionenstrahl bzw. (b) mit gerastertem Ionenstrahl bestrahlten Si(001)-Substrats. Die Graphen zeigen das jeweilige Linienprofil entlang der im Bild gekennzeichneten Strecke. 
der Rippelwellenlänge beobachtet und dies auf eine stärkere Erwärmung der Probe bei statischem Ionenstrahl sowie resultierender Dominanz thermischer Oberflächendiffusion zurückgeführt. Diese Argumentation wird durch das Bradley-Harper-Modell, welche ausschließlich thermisch aktivierte Oberflächendiffusion berücksichtigt und eine entsprechende Abnahme der Wellenlänge mit ansteigender Energie voraussagt, gestützt. Kontrovers hierzu äußert sich indes die ferner beobachtete Unabhängigkeit von der Ionenstromdichte.

Abgesehen von den zum Abschluss dieses Kapitels dargestellten Ergebnissen (Abschnitt 4.2.2.3 und 4.2.2.4) der in der Ionenmühle unter Stickstoffkühlung bestrahlten Substrate wurden alle im Folgenden beschriebenen Proben in der UHV-Anlage bei gerastertem Ionenstrahl hergestellt.

\subsubsection{Winkelabhängigkeit}

Die Winkelabhängigkeit der RMS-Rauigkeit unter $0.8 \mathrm{keV} \mathrm{Ar}^{+}$-Bestrahlung (Abbildung 4.7(e)) zeigt ein vergleichbares Verhalten wie unter $\mathrm{Kr}^{+}$-Bestrahlung (Abschnitt 4.2.1.2), das sich in Anlehnung an den Sputter-Yield für nicht zu streifenden Ioneneinfall ebenfalls gut durch ein $\sigma_{\mathrm{RMS}}(\theta)=\mathrm{a}_{1}+\mathrm{a}_{2} / \cos ^{\mathrm{q}}(\theta)$-Gesetz mit $\mathrm{q} \approx 4$ beschreiben lässt. Mit zunehmendem Winkel ist außerdem eine deutlichere Ausprägung länglicher Wellenstrukturen zu beobachten (Abbildung 4.7(a)-(d)).

Zwecks Charakterisierung hinsichtlich des Ordnungsgrades auftretender Rippel wurde die parallel zum Wellenvektor der Korrugationen gemittelte spektrale Leistungsdichte (PSD) bestimmt. Die Peak-Position des Maximums erster Ordnung gibt hierbei Auskunft über die dominierende Wellenlänge, während dessen Halbwertsbreite B eine Quantifizierung der lateralen Ordnung erlaubt. B soll daher im Weiteren auch als Ordnungsparameter bezeichnet werden. Störungen der Rippelstruktur führen auf Grund zusätzlicher Frequenzen $\mathrm{zu}$ einer entsprechenden Verbreiterung des Maximums. Eine wohl definierte Rippelstruktur zeichnet sich folglich durch einen niedrigen Ordnungsparameter aus. Die zur quantitativen Beschreibung verwendeten statistischen Kenngrößen sind in Tabelle 4.1 zusammengestellt.

Neben dem Anstieg der Rauigkeit und zunehmender Ordnung ist für große Winkel $\theta$ (Abbildung 4.7(c)-(d)) eine in Strahlrichtung breiter werdende dreieckige Struktur erhöhter Bereiche erkennbar, die in Bezug auf die mittlere Oberflächenebene leicht gegen den Strahl geneigt ist. Ähnliche Beobachtungen wurden von Alkemade und Jiang [Alk01] unter Verwendung von $\mathrm{O}_{2}{ }^{+}$-Ionen bei vergleichbarer Energie berichtet, ohne jedoch die Ursache zu klären. Denkbar ist eine wachsende Instabilität, die infolge des streifenden Ioneneinfalls ihren Ursprung in Abschattungseffekten an Erhebungen der schon anfänglich rauen Oberfläche besitzt. Der Öffnungswinkel im Scheitelpunkt der in Abbildung 4.7(c)-(d) sichtbaren Dreiecke beträgt sowohl für $\theta=70^{\circ}$ als auch für $\theta=75^{\circ} 33(2)^{\circ}$ und besitzt eine auffällige Konformität ohne merkliche Streuung. Obwohl angenommen wird, dass sich die Siliziumoberfläche durch ionenstrahlinduzierte Amorphisierung isotrop verhält, offenbaren Un- 
(a) $\theta=45^{\circ}$

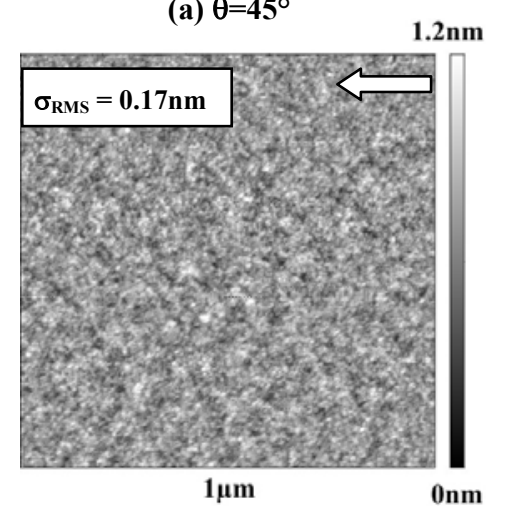

(d) $\theta=\mathbf{7 5}^{\circ}$

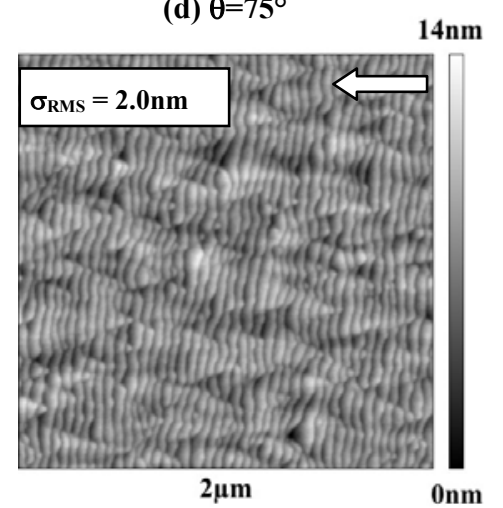

(b) $\theta=65^{\circ}$

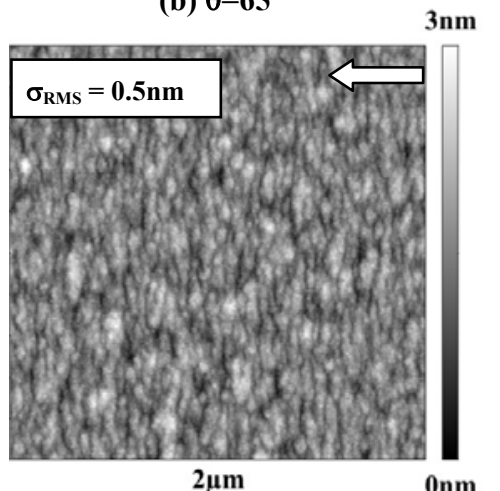

(c) $\theta=70^{\circ}$

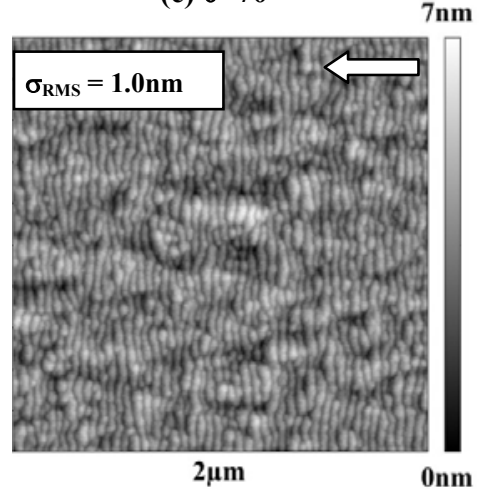

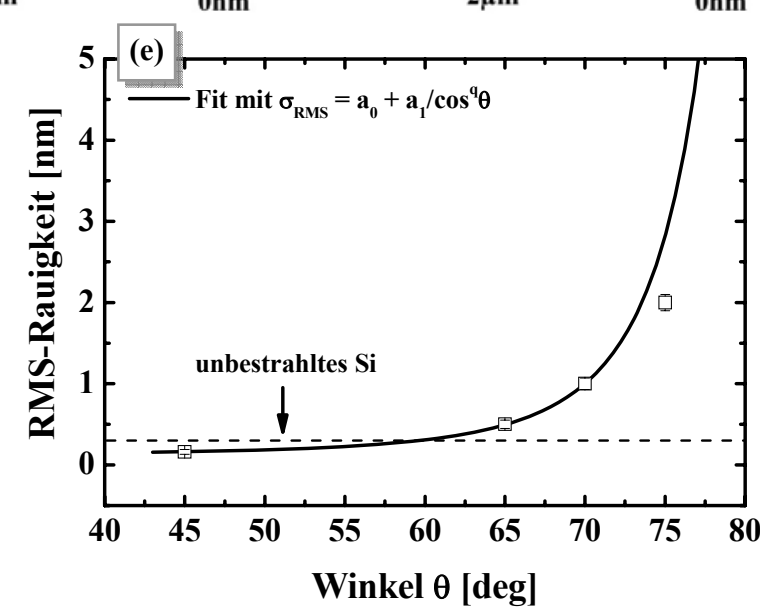

Abbildung 4.7: (a)-(d) AFM-Aufnahmen von mit 0.8keV $\mathrm{Ar}^{+}$bestrahlten $\mathrm{Si}(001)-$ Substraten bei unterschiedlichen Einfallswinkeln und einer Bestrahlungsdauer von 48h sowie (e) Winkelabhängigkeit der RMS-Rauigkeit.

\begin{tabular}{|c|c|c|c|c|}
\hline $\begin{array}{c}\text { Winkel } \\
\theta[\mathrm{deg}]\end{array}$ & $\begin{array}{c}\text { Wellenlänge } \\
\lambda[\mathrm{nm}]\end{array}$ & $\begin{array}{c}\text { Rippeltiefe } \\
\mathrm{d}_{\mathrm{R}}[\mathrm{nm}]\end{array}$ & $\begin{array}{c}\text { Ordnungsparameter } \\
\mathrm{B}\left[\mathrm{nm}^{-1}\right]\end{array}$ & $\begin{array}{c}\text { Rauigkeit } \\
\sigma_{\mathrm{RMS}}[\mathrm{nm}]\end{array}$ \\
\hline 45 & - & - & - & 0.17 \\
65 & $\sim 90^{(\mathrm{a})}$ & $\sim 1$ & $>0.1^{(\mathrm{a})}$ & 0.5 \\
70 & 45 & 1.5 & 0.070 & 1.0 \\
75 & 51 & 2.5 & 0.046 & 2.0 \\
\hline
\end{tabular}

Tabelle 4.1: Statistische Kenndaten der mit $0.8 \mathrm{keV} \mathrm{Ar}^{+}$bestrahlten Si-Substrate.

(a) Für $\theta=65^{\circ}$ ist das Maximum der PSD nicht deutlich ausgeprägt.

tersuchungen von Hazra und Chini et al. [Haz04, Chi03] eine zur Oberfläche korrelierte Rippelstruktur der Grenzfläche zwischen amorpher und kristalliner Phase. Takahiro et al. [Tak07] konnten an unterschiedlichen Formen des Kohlenstoffs (Diamant, Graphit und amorpher Kohlenstoff), die alle eine oberflächlich amorphisierte Phase vergleichbarer Dichte ausbildeten, eine deutliche Auswirkung des zugrunde liegenden Materials auf die resultierende Oberflächenmorphologie demonstrieren. Demzufolge ist ein Einfluss der Kristallstruktur zwar unwahrscheinlich, aber unter spezifischen Bestrahlungsbedingungen 
auch hier nicht von vornherein auszuschließen, wobei die Überprüfung durch Variation der Substratausrichtung relativ zum projizierten Ionenstrahl unter Beibehaltung der verwendeten Bestrahlungsparameter noch aussteht.

\subsubsection{Energieabhängigkeit}

Da die strahlungsinduzierten Glättungs- und Aufrauungsmechanismen in erheblichem Maße von der Energiedeposition im Target beeinflusst werden, stellt die Ionenenergie einen wesentlichen Parameter bei der Strukturbildung dieser konkurrierenden Prozesse dar. Abbildung 4.8 zeigt die Oberflächentopographie nach einer Bestrahlungsdauer von $24 \mathrm{~h}$ bzw. $48 \mathrm{~h}$ unter $75^{\circ}$ mit unterschiedlicher Energie. Die entsprechenden statistischen Kenndaten sind in Tabelle 4.2 aufgeführt. Für $0.6 \mathrm{keV}$ entwickelt sich zunächst eine Rippelstruktur geringer lateraler Ordnung, die sich im Zuge weiterer Bestrahlung stärker ausprägt (Abbildung 4.8(a)-(b)). Mit zunehmender Ionenenergie kommt es zur Abnahme des Ordnungsparameters. Die mit $0.8 \mathrm{keV}$ bestrahlte Probe (Abbildung 4.8(c)) zeichnet sich hierbei besonders aus, da sie einerseits die höchste Rauigkeit, gleichzeitig jedoch den höchsten Ordnungsgrad besitzt (Abbildung 4.9(b)). Abgesehen von der auffällig geglätteten Oberfläche bei $2.3 \mathrm{keV}$ (Abbildung 4.8(e)) zeigen alle Proben mehr oder weniger signifikante dreieckige Strukturen mit Scheitelwinkel von $33^{\circ}$. Diese Störung wird hier als maßgebliche Einschränkung der transversalen Ausdehnung geordneter Korrugationen identifiziert. Ähnlich wie bei der zuvor betrachteten Winkelabhängigkeit (Abschnitt 4.2.2.1) lässt die Energieabhängigkeit oberhalb einschließlich $2.3 \mathrm{keV}$ ebenfalls einen Bereich dominierender Glättungsmechanismen erkennen, wobei die Wellenlänge in Bezug auf die Energie nahezu linear verläuft (Abbildung 4.9(a)).

\begin{tabular}{|c|c|c|c|c|c|}
\hline $\begin{array}{c}\text { Energie } \\
\mathrm{E}[\mathrm{keV}]\end{array}$ & $\begin{array}{c}\text { Bestrahlungs- } \\
\text { dauer } \mathrm{t}[\mathrm{h}]\end{array}$ & $\begin{array}{c}\text { Wellenlänge } \\
\lambda[\mathrm{nm}]\end{array}$ & $\begin{array}{c}\text { Rippeltiefe } \\
\mathrm{d}_{\mathrm{R}}[\mathrm{nm}]\end{array}$ & $\begin{array}{c}\text { Ordnungsparameter } \\
\mathrm{B}\left[\mathrm{nm}^{-1}\right]\end{array}$ & $\begin{array}{c}\text { Rauigkeit } \\
\sigma_{\mathrm{RMS}}[\mathrm{nm}]\end{array}$ \\
\hline 0.6 & 24 & 40 & 2.5 & 0.077 & 1.2 \\
0.6 & 48 & 42 & 2.5 & 0.058 & 1.3 \\
0.8 & 48 & 51 & 2.5 & 0.046 & 2.0 \\
1.0 & 48 & 60 & 1.5 & 0.064 & 1.0 \\
2.3 & 48 & $\sim 100^{(\mathrm{a})}$ & $<0.5$ & $>0.1^{(\mathrm{a})}$ & 0.2 \\
\hline
\end{tabular}

Tabelle 4.2: Statistische Kenndaten der unter $\theta=75^{\circ}$ bestrahlten Si-Substrate.

${ }^{\text {(a) }}$ Für $\mathrm{E}=2.3 \mathrm{keV}$ ist das Maximum der PSD nicht deutlich ausgeprägt.

Wellenlänge: Wird allein thermische Oberflächendiffusion als Glättungsmechanismus berücksichtigt, ist die Energieabhängigkeit der Wellenlänge im Rahmen des BradleyHarper-Modells nach Gleichung 4.5 durch 
(a) $\mathrm{E}=0.6 \mathrm{keV}, \mathrm{t}=\mathbf{2 4 h}$

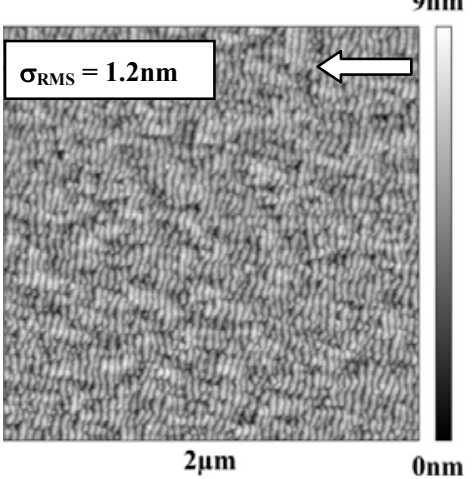

(d) $\mathrm{E}=1.0 \mathrm{keV}, \mathrm{t}=48 \mathrm{~h}$ (b) $\mathrm{E}=0.6 \mathrm{keV}, \mathrm{t}=48 \mathrm{~h}$

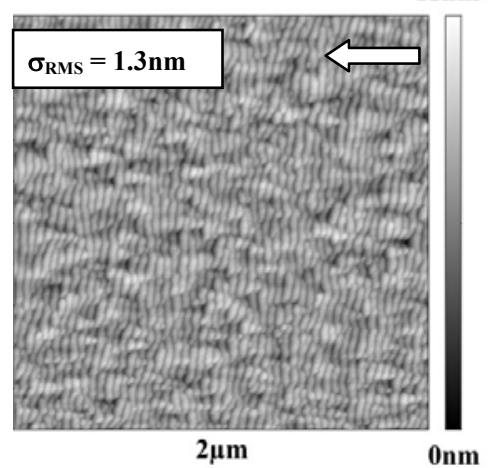

(e) $\mathrm{E}=2.3 \mathrm{keV}, \mathrm{t}=48 \mathrm{~h}$ (c) $\mathrm{E}=0.8 \mathrm{keV}, \mathrm{t}=48 \mathrm{~h}$

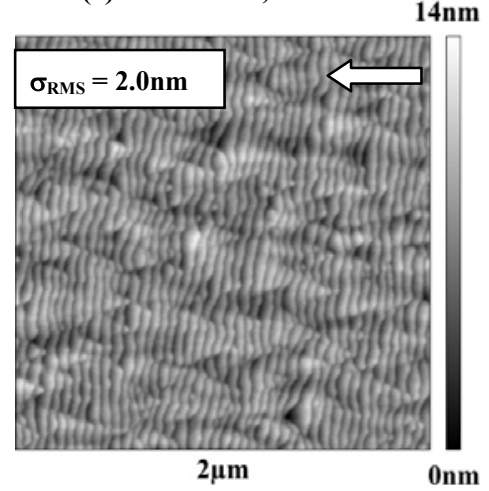

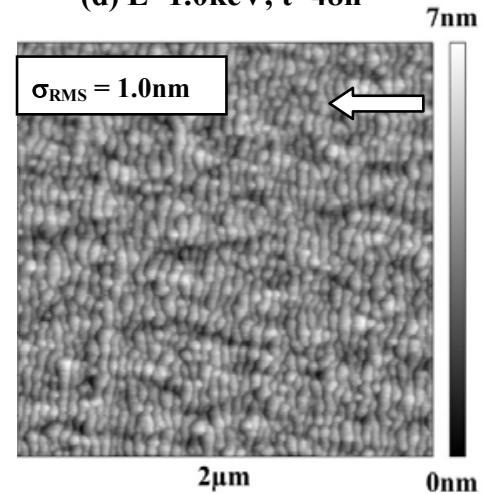

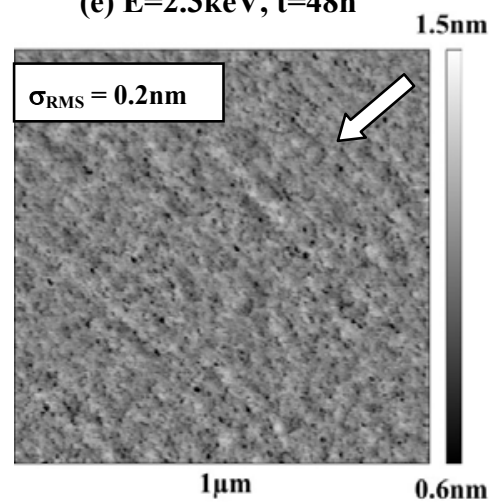

Abbildung 4.8: AFM-Aufnahmen von mit $\mathrm{Ar}^{+}$-Ionen unterschiedlicher Energie bestrahlten $\operatorname{Si}(001)$-Substraten unter einem Winkel von $75^{\circ}$ und einer Bestrahlungsdauer $\mathrm{t}$ von $24 \mathrm{~h}$ bzw. $48 \mathrm{~h}$.
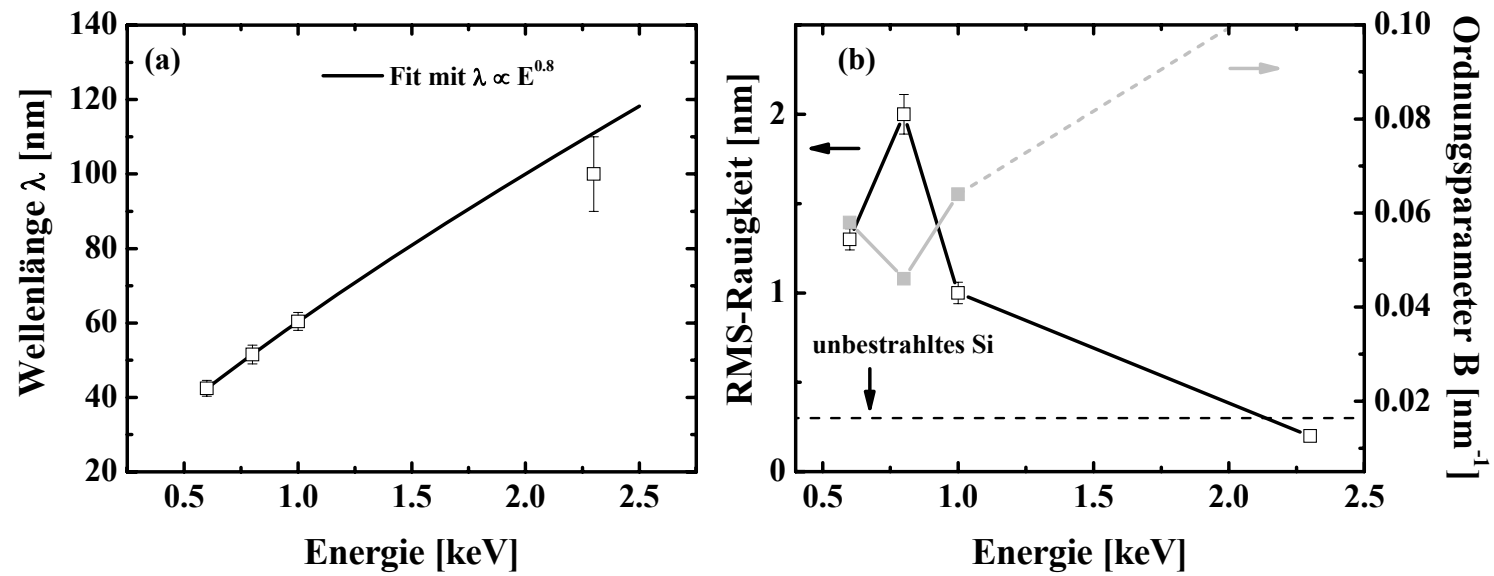

Abbildung 4.9: (a) Energieabhängigkeit der Wellenlänge sowie (b) RMSRauigkeit und Ordnungsparameter von mit $\mathrm{Ar}^{+}-$Ionen bestrahltem Si unter einem Winkel von $75^{\circ}$ und einer Bestrahlungsdauer von $48 \mathrm{~h}$. 


$$
\lambda(E)=2 \pi \sqrt{\frac{2 n \cdot K}{f \cdot a(E) \cdot Y_{0}(E) \cdot|\Gamma(E)|}}
$$

gegeben. Nach Simulationen mittels des Programms SRIM gehorchen die mittlere Tiefe der Energiedeposition a(E) sowie der Sputter-Yield für den hier untersuchten Energiebereich unter einem Winkel von $75^{\circ}$ etwa einem $\mathrm{a}(\mathrm{E}) \propto \mathrm{E}^{0.6}$ - bzw. einem $\mathrm{Y}_{0}(\mathrm{E}) \propto \mathrm{E}^{0.6}-$ Verhalten. Die nur schwache Energieabhängigkeit der Funktion $\Gamma$ über die Quotienten $a(E) / \alpha(E)$ bzw. a(E) $/ \beta(E)$ kann vernachlässigt werden [Cha07]. Hieraus ergibt sich für die Wellenlänge ein Potenzgesetz

$$
\lambda(E) \propto E^{p}
$$

mit $\mathrm{p}<0$ und somit eine mit der Energie abnehmende Wellenlänge, wie sie z.B. von Brown und Erlebacher [Bro05a] für hohe Temperaturen beobachtet wurde. Bestrahlungen bei Raumtemperatur zeigen indes häufig ein Potenzgesetz mit positivem Exponenten zwischen 0.8 und 1 [Vaj96, Dat01].

Wie in Abschnitt 4.1 erläutert, wurden die Modelle zur Beschreibung der Oberflächenentwicklung durch zusätzliche Mechanismen erweitert. Ionenstrahlinduziertes viskoses Oberflächenfließen trägt gleichermaßen zum $\vec{\nabla}^{4} \mathrm{~h}$-Term bei, besitzt hingegen über die Energiedepositionstiefe eine energieabhängige Relaxationsrate $K(E) \propto a^{3}(E)$ und nach [Umb01] bedeutet dies für die Wellenlänge:

$$
\lambda(E) \propto a(E) \sqrt{\frac{1}{Y_{0}(E) \cdot|\Gamma(E)| \cdot \eta_{r}(E)}},
$$

wobei $1 / \eta_{\mathrm{r}} \propto \mathrm{E}$ ein Maß für die viskose Relaxation pro Ion darstellt. Anhand der oben genannten Simulationsergebnisse folgt somit aus Gleichung 4.9 ein Exponent von $\mathrm{p}=0.8$. Der in Anbetracht des stark fehlerbehafteten Wertes für $2.3 \mathrm{keV}$ nahezu lineare Verlauf der Wellenlänge (Abbildung 4.9(a)) ist folglich ein Anzeichen für die Relevanz des Oberflächenfließens [Umb01] auch unter schrägem Ioneneinfall von hier $75^{\circ}$ und bekräftigt die von Vauth und Mayr [Vau07] gezeigte Dominanz gegenüber Diffusionsprozessen.

Rauigkeit und Ordnung: Das Kontinuumsmodell, basierend auf dem Bradley-HarperModell und genannter Erweiterungen, erlaubt Aussagen über die durch konkurrierende Aufrauungs- und Glättungsmechanismen dominierenden und somit am schnellsten anwachsenden Pertubationen entlang oder senkrecht zum projizierten Ionenstrahl. Meist handelt es sich hierbei um einen sehr schmalen Frequenzbereich, der dann vornehmlich die Oberflä- 
chenmorphologie bestimmt und eine Rippelstruktur ausbildet. Die Rauigkeit der Oberfläche ist demzufolge direkt mit der Tiefe dieser Wellenformation verbunden. Experimentell wird häufig eine mit der Wellenlänge der Rippel ansteigende Rippeltiefe und Rauigkeit beobachtet [Fla01]. Mögliche Ursachen für die stärkere Limitierung der Amplitude bei kleinen Wellenlängen sind nichtlineare Effekte sowie die Geometrie, die bei streifendem Ioneneinfall auf Grund von Abschattungseffekten nur eine begrenzte Amplitude zulässt.

Das Zusammentreffen maximaler Ordnung und Rauigkeit in Abbildung 4.9(b) ohne deutlichen Anstieg der Rippeltiefe widerspricht dem oben erläuterten Verhalten und liegt in der transversalen Störung begründet. Während die Größe der dreieckigen Bereiche und die Regelmäßigkeit der Wellen innerhalb dieser Bereiche zu $0.8 \mathrm{keV}$ hin zunimmt, wächst gleichzeitig deren Höhenunterschied, wodurch eine zusätzliche Rauigkeit vermittelt wird. Die deutliche Abnahme der Rippeltiefe für $1 \mathrm{keV}$ und $2.3 \mathrm{keV}$ lässt sich hiermit hingegen nicht erklären. Auch die Energieabhängigkeit des Sputter-Yields kann als Ursache ausgeschlossen werden, da die Abnahme des Sputter-Yields infolge höherer Ioneneindringtiefe und geringerer oberflächennaher Energiedeposition für senkrechten $\mathrm{Ar}^{+}$-Ioneneinfall erst bei deutlich höheren Energien ( $>20 \mathrm{keV}$ [Sig69]) beobachtet wird. Mit zunehmendem Winkel $\theta$ und damit geringerer Ioneneindringtiefe sollte sich demnach das Maximum des Sputter-Yields zu noch höheren Energien verschieben. Jiang und Alkemade [Jia98] berichteten über eine ähnlich ungewöhnliche Aufrauung für Ionenenergien $\mathrm{E} \leq 1 \mathrm{keV}$ und nur geringe Änderung der Rauigkeit für $2 \mathrm{keV}$, spekulierten jedoch in diesem Zusammenhang über die Auswirkung lokal inhomogener Sauerstoffimplantation, verursacht durch das von ihnen verwendete reaktive Ionengas $\mathrm{O}_{2}^{+}$.

Die Vielzahl unterschiedlicher experimentell gewonnener Erkenntnisse zeigt, dass die theoretische Beschreibung auftretender Rippelstrukturen noch immer Lücken aufweist und die für die Anwendung dieses Strukturierungsverfahrens erforderliche genaue Kenntnis bezüglich des Einflusses äußerer Parameter bislang nur in Ansätzen vorhanden ist.

\subsubsection{Hochgradig geordnete Wellenstrukturen}

Für die mittels statischen Ionenstrahls in der Ionenmühle hergestellten Proben wurde um den mit bloßem Auge sichtbaren Bereich des maximalen Abtrags herum eine über weite Strecken vergleichbare Oberflächenmorphologie beobachtet.

Abbildung 4.10 zeigt die unterschiedliche Strukturbildung im Randbereich und im Intensitätsmaximum des eintreffenden Ionenstrahls nach einer Bestrahlungsdauer von $12 \mathrm{~h}$. Neben der (aus den beiden vorhergehenden Abschnitten) bereits bekannten Ausbildung triangulärer Erhebungen entwickelt sich eine zusätzliche überlagerte Korrugation größerer Wellenlänge, die für hohe Fluenzen (Abbildung 4.10(c)-(d)) eine sägezahnförmige, facettierte Gestalt mit kurzwelligerer Rippelstruktur auf der dem Strahl zugewandten Seite und glatter dem Strahl abgewandter Flanke annimmt. Die glatte Flanke besitzt einen Winkel 

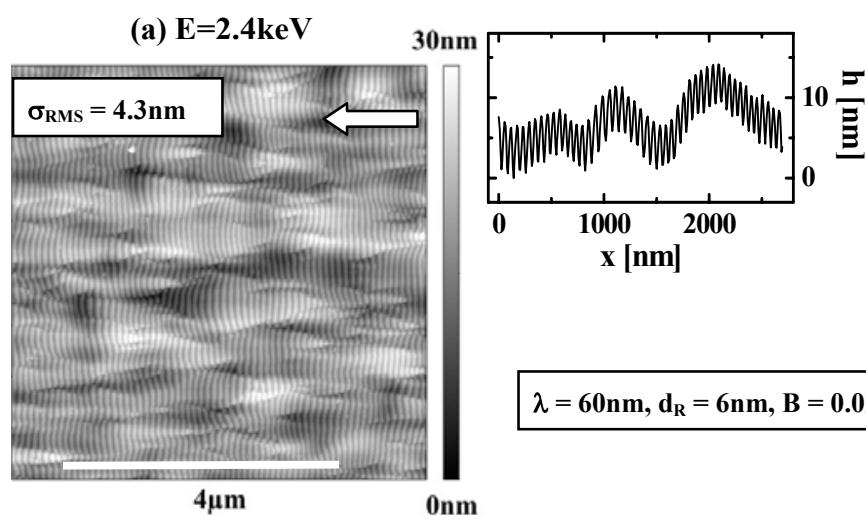

$\lambda=60 \mathrm{~nm}, \mathrm{~d}_{\mathrm{R}}=6 \mathrm{~nm}, \mathrm{~B}=0.020 \mathrm{~nm}^{-1}$

(c) $\mathrm{E}=2.4 \mathrm{keV}$

$75 \mathrm{~nm}$
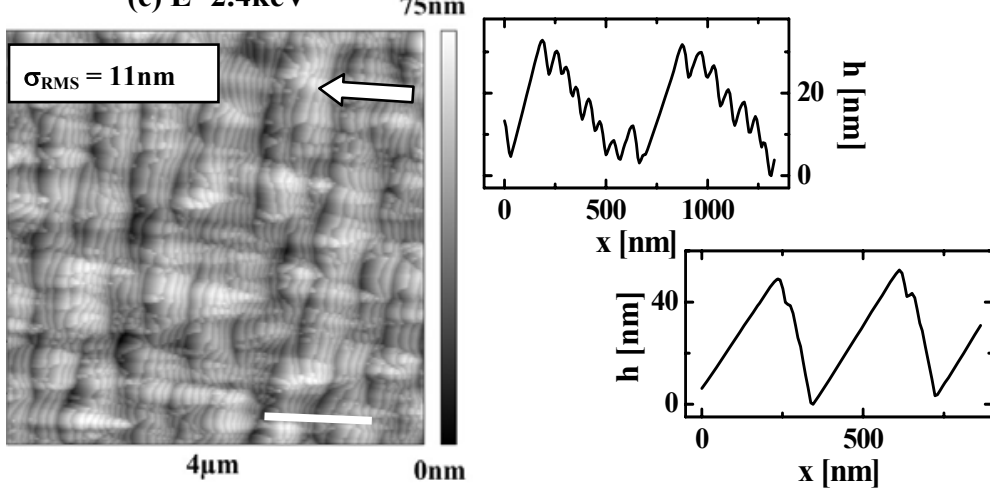

(b) $\mathrm{E}=2.4 \mathrm{keV}$

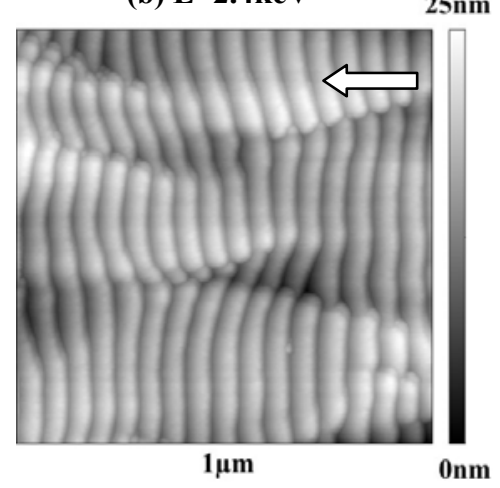

(d) $\mathrm{E}=3.0 \mathrm{keV}$

$130 \mathrm{~nm}$

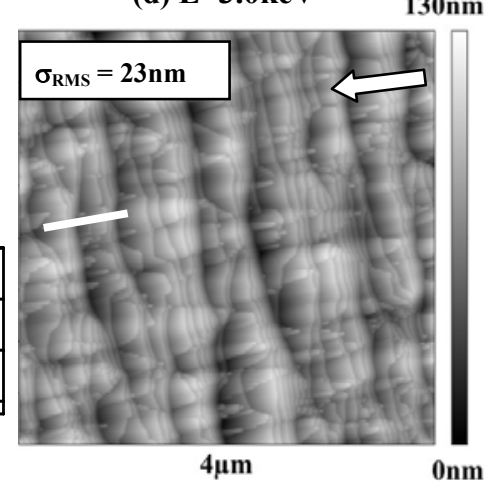

Abbildung 4.10: AFM-Aufnahmen der mittels statischen Ionenstrahls unter einem Winkel von $70^{\circ}$ und $12 \mathrm{~h}$ Bestrahlungsdauer hergestellten Proben. (a) und (b) wurden im Randbereich, (c) und (d) bei unterschiedlicher Energie im Intensitätsmaximum aufgenommen.
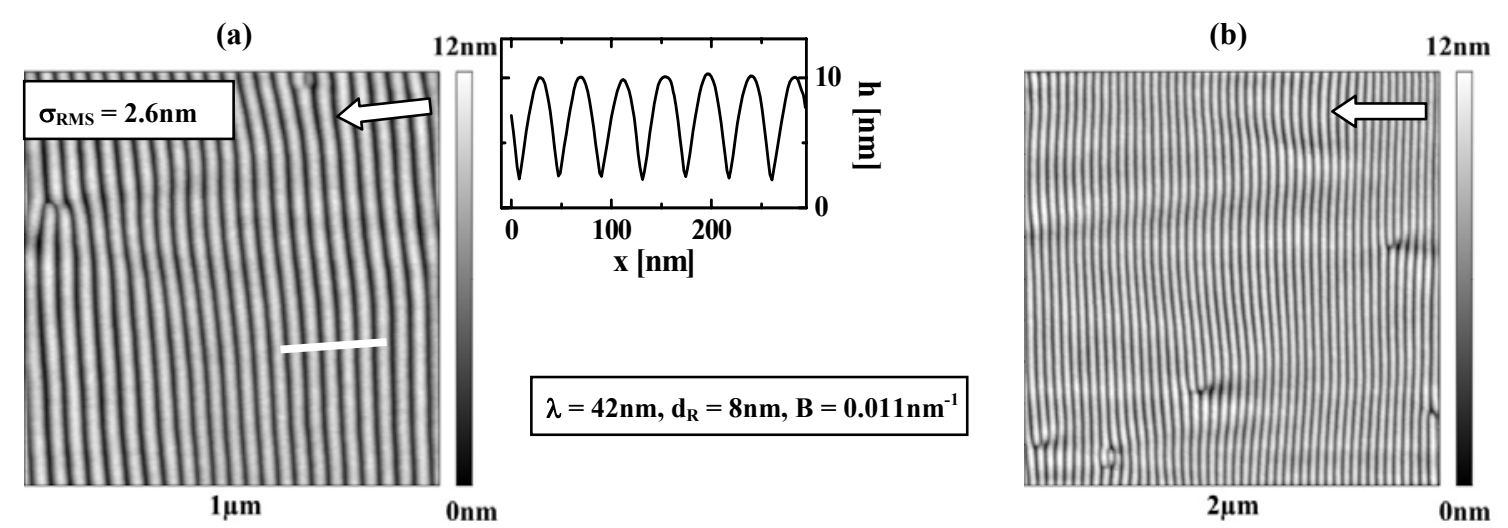

Abbildung 4.11: AFM-Aufnahmen im Randbereich einer mit 3.8keV unter $\theta=$ $60^{\circ}$ bestrahlten Probe nach einer Bestrahlungsdauer von 6h. (a) und (b) zeigen unterschiedlich große Bildausschnitte. 
von nur etwa $10^{\circ}$ relativ zur Richtung des einfallenden Ionenstrahls und weist daher keine senkrecht hierzu ausgerichtete Wellenstruktur auf.

$\mathrm{Ob}$ die in dreieckigen Formen resultierende Störung im kausalen Zusammenhang mit der auf Grund des hohen Aspektverhältnisses durch Abschattungseffekte für hohe Fluenzen facettierten Überstruktur steht, kann hier nicht vollständig geklärt werden. Sie ist jedoch offensichtlich direkt verantwortlich für die Entstehung von Versetzungsrippeldefekten (Abbildung 4.10(b)), bei denen sich zwei Wellenberge zu einem zusammenschließen bzw. sich eine Fehlpassung aneinander angrenzender geordneter Bereiche ausbildet.

Wesentlichster Unterschied zu den in der UHV-Anlage bestrahlten Substraten ist die, wenn auch nicht genau messbare, deutlich höhere Ionenstromdichte sowie die Kühlung mittels flüssigen Stickstoffs, wodurch thermisch induzierte Relaxationen von Oberflächenkorrugationen infolge einer Probenerwärmung weitestgehend ausgeschlossen werden können. Die hier bei geringerer Bestrahlungsdauer dennoch vergleichsweise höhere Fluenz legt nahe, dass sich die Oberflächenmorphologie bereits im Einfluss nichtlinearer Terme befindet, wie dies anhand sich stabilisierender Rippelamplitude üblicherweise für Fluenzen $\Phi \geq 10^{18} / \mathrm{cm}^{2}$ beobachtet wird [May05].

Durch Verringerung der Bestrahlungsdauer auf $\mathrm{t}=6 \mathrm{~h}$ und kleinerem Einfallswinkel $\theta=$ $60^{\circ}$ konnten bei einer Ionenenergie von $3.8 \mathrm{keV}$ Rippelsubstrate einer hochgradig geordneten Wellenstruktur mit deutlich reduziertem Ordnungsparameter von $B=0.011 \mathrm{~nm}^{-1} \mathrm{er}$ zeugt werden (Abbildung 4.11), welche letztlich als Templates für die nachfolgenden Untersuchungen der Selbstorganisation deponierter Ni- und NiAg-Schichten eingesetzt wurden. Die Defektdichte wurde unter Auswertung mehrerer AFM-Aufnahmen zu etwa $1.5 \mu \mathrm{m}^{-2}$ bestimmt, wobei die Entfernung zweier aufeinander folgender Defekte entlang einer Furche weit über $4 \mu \mathrm{m}$ liegt. Die im Linienprofil der Abbildung 4.11 ersichtlichen schmalen Täler resultieren aus einem Messartefakt, hervorgerufen durch den hohen Krümmungsradius der AFM-Spitze (Abbildung 2.9).

Geometrie der geordneten Rippelstruktur: Anhand geometrischer Überlegungen lässt sich zeigen, dass das beobachtete Verhältnis $\left(\pi \cdot \mathrm{d}_{\mathrm{R}} / \lambda=0.60\right)$ mittlerer Rippeltiefe $d_{R}=8 \mathrm{~nm}$ zur Wellenlänge $\lambda=42 \mathrm{~nm}$ etwa dem für sinusförmige Wellen maximalen Aspektverhältnis unter Ioneneinfallswinkel von $\theta=60^{\circ}$ entspricht. Wird der Wert $\pi \cdot d_{R} / \lambda=\tan \left(90^{\circ}-\theta\right)=0.58$ überschritten, kommt es infolge großer Steigung an der dem Strahl abgewandten Seite zu Abschattungseffekten (Abbildung 4.12). Eine Analyse

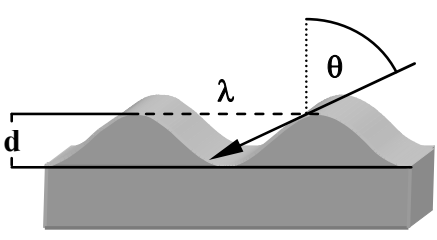

Abbildung 4.12: Geometrische Abschattung bei hohem Aspektverhältnis $\mathrm{d} / \lambda$. der Verteilungsfunktion lokaler Oberflächenwinkel (Abbildung 4.13) offenbart, dass keine streng sinusförmige Wellenstruktur mit um 0 symmetrischer Winkelverteilung vorliegt, sondern eine geringe Verschiebung zu negativen Steigungen auftritt, was auf eine leicht in 


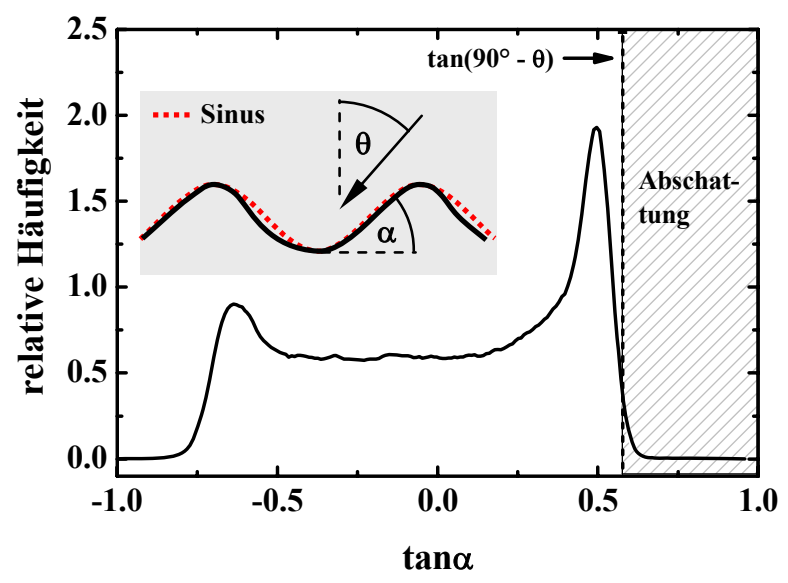

Abbildung 4.13: Winkelverteilung der mit 3.8keV $\mathrm{Ar}^{+}$-Ionen unter $\theta=60^{\circ}$ bestrahlten Oberfläche.

den Strahl geneigte Flanke zurückzuführen ist. Die unterschiedliche Ausprägung lokaler Maxima relativer Häufigkeit positiver bzw. negativer Steigungen deutet auf eine abgeflachte, dem Strahl abgewandten Flanke hin, wodurch vermehrt dieselbe Steigung entsteht, und geringer Abflachung der dem Strahl zugewandten Flanke. Die Geometrie der Rippelstruktur zeichnet sich somit durch eine größtmögliche Rippeltiefe bei nur schwacher Asymmetrie aus, die die geforderten Bedingungen einer kleinen Wellenlänge bei maximal erreichbarer Krümmung und hoher Ordnung zur Anwendung als Templates erfüllt.

Experimentelle Untersuchungen sowie theoretische Betrachtungen haben sich vornehmlich auf das Verständnis der Dynamik sich ausbildender Rippel konzentriert und die Frage der technisch relevanten lateralen Ordnung weitestgehend außer Acht gelassen. Hier konnte gezeigt werden, dass die transversale Ausdehnung der Wellenberge hochgradig geordneter Rippel maßgeblich von einer sich in dreieckigen Strukturen mit Scheitelwinkel von 33(2) ${ }^{\circ}$ äußernden Störung beeinträchtigt wird, deren Ursprung jedoch ungeklärt ist. Möglich ist der Einfluss von Abschattungseffekten, die für große Einfallswinkel und hohe Bestrahlungszeiten in zunehmendem Maße die sinusförmige Gestalt der Wellen stören. Auf Grund von räumlich begrenzten Intensitätsspitzen im stochastischen Rauschen des stationären Ionenstrahls kann es dabei zu Inhomogenitäten kommen, die diesen Effekt lokal verstärken und letztlich $\mathrm{zu}$ in Strahlrichtung breiter werdenden signifikanten Bereichen führen. Die scheinbare Unabhängigkeit des Scheitelwinkels vom Einfallswinkel $\theta$ schließt jedoch eine rein geometrische Ursache aus und suggeriert die Relevanz zusätzlicher Mechanismen. Für nahe senkrechten Ioneneinfall ist zwar Abschattung bis zu großen Rippeltiefen ausgeschlossen, die Dominanz der Oberflächenglättung erlaubt indes keine Entwicklung periodischer Korrugationen ausgeprägtem Aspektverhältnisses. 


\subsubsection{Tiefe Wellenstrukturen}

Um den Einfluss gröberer Strukturen auf den Selbstorganisationsprozess während der NiAg-Deposition zu untersuchen, wurden außerdem Substrate unter einem Winkel von $\theta=$ $85^{\circ}$ für $6 \mathrm{~h}$ bestrahlt. Hierbei entstehen tiefe Wellenstrukturen mit paralleler Ausrichtung zum projizierten Ionenstrahl (Abbildung 4.14). Die Korrugationen sind jedoch weitestgehend ungeordnet und besitzen keine wohl definierte Wellenlänge. Anhand des Linienprofils ist ersichtlich, dass die Oberfläche neben ausgeprägter Rillen mit einer Tiefe bis zu 200nm auch deutlich feinere Pertubationen aufweist.

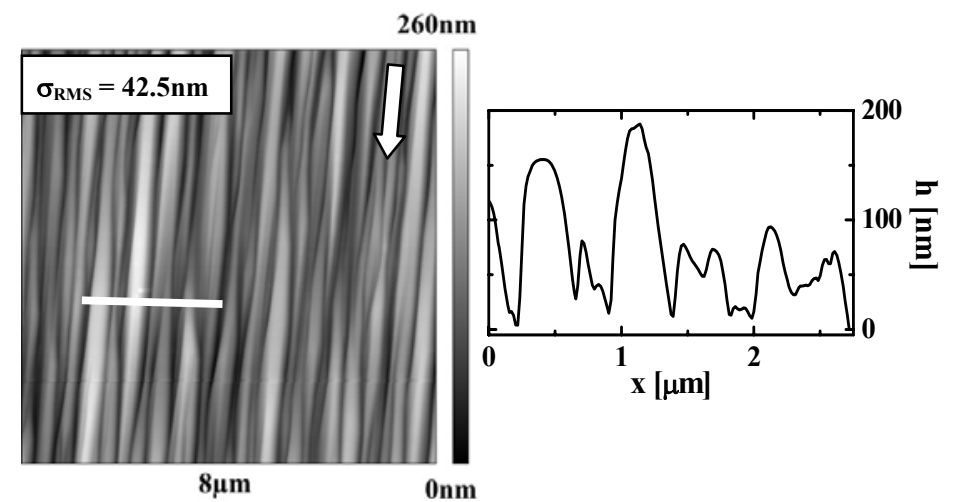

Abbildung 4.14: AFM-Aufnahme und Linienprofil eines unter $\theta=85^{\circ}$ mit einer Energie von $4.2 \mathrm{keV}$ für $6 \mathrm{~h}$ bestrahlten Substrats.

\subsubsection{Thermische Oxidation}

Zwecks chemischer Stabilisierung der Oberfläche, um Interdiffusion der deponierten Schichten und dem Substrat im Rahmen weiterer Untersuchungen zu vermeiden, wurden die strukturierten Proben in ein mit Aceton gereinigtes, ausgeheiztes, nicht luftdicht verschlos-
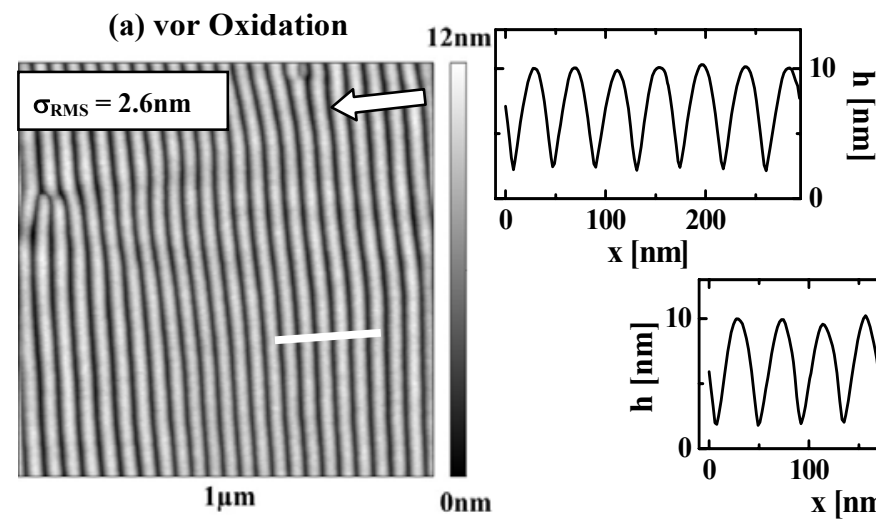

(b) nach Oxidation

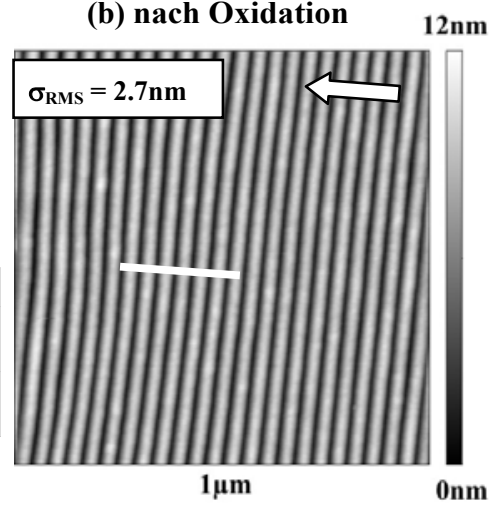

Abbildung 4.15: AFM-Aufnahmen der geordneten Rippelstruktur (a) vor und (b) nach thermischer Oxidation. 


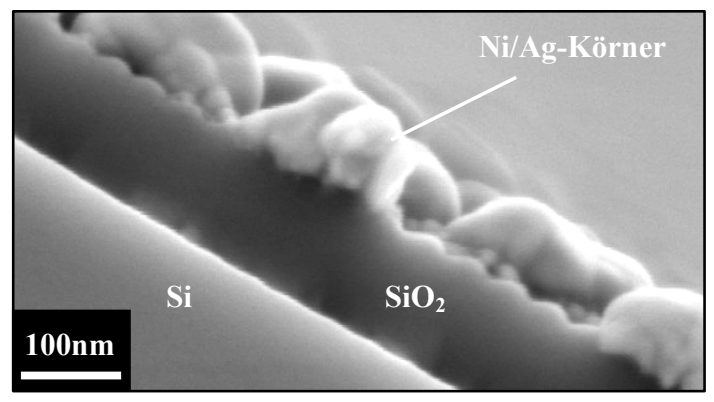

Abbildung 4.16: REM-Querschnittsaufnahme der Bruchkante eines thermisch oxidierten, rippelstrukturierten Substrats. Die Ni/Ag-Körner sollen an dieser Stelle außer Acht gelassen werden.

senes Quarzgefäß gelegt und im Ofen bei $1080^{\circ} \mathrm{C}$ für drei Stunden thermisch oxidiert. Hierbei bleiben die spezifischen Charakteristika der Oberflächenkorrugationen im Wesentlichen erhalten. AFM-Aufnahmen (Abbildung 4.15) zeigen keine merkliche Veränderung der Rippelstruktur nach der thermischen Behandlung. Die Dicke der Oxidschicht wurde mittels REM-Querschnittsaufnahmen (Abbildung 4.16) an der Bruchkante eines entsprechend strukturierten Substrats zu $>100 \mathrm{~nm}$ bestimmt, was ausreichend ist, um eine Diffusion von Nickel ins Silizium zu unterbinden [May92]. 



\section{Kapitel}

\section{Selbstorganisation während der Deposition}

Die Entwicklung polykristalliner Filme auf glatten Substratoberflächen infolge stochastischer atomarer Materialdeposition wird für gewöhnlich durch die Nukleation regellos angeordneter isolierter Keime von Adsorbatatomen initiiert. Mit zunehmender Materialmenge kommt es zum Wachstum der Kristallite, wobei laterales Wachstum die Koaleszenz benachbarter Kristalle bis hin zur Ausbildung einer geschlossenen Schicht ermöglicht. In diesem Kapitel soll zunächst der Fragestellung nachgegangen werden, ob eine wellenförmige Substratoberfläche die atomaren Mechanismen der Kristallbildung und -organisation in dem Maße beeinflusst, dass eine kompositionelle sowie geometrische Ordnung während der Kodeposition von dünnen NiAg-Schichten induziert werden kann. Die strukturelle Analyse stützt sich dabei überwiegend auf REM-Untersuchungen der Probenoberflächen, die Aufschluss über die laterale Materialverteilung geben.

Wie bereits zuvor erwähnt wurde, sind Nickel und Silber selbst in flüssiger Phase nicht mischbar und das System besitzt mit $\Delta \mathrm{H}=23 \mathrm{~kJ} / \mathrm{mol}$ [Boe88] eine vergleichsweise hohe Mischungsenthalpie. Um einen entsprechend zu erwartenden Separationsprozess sowie die Kinetik der Oberflächenprozesse voranzutreiben, wurde während der Deposition eine erhöhte Substrattemperatur von $600^{\circ} \mathrm{C}$ verwendet.

Nickel weist mit $\gamma_{\mathrm{Ni}}=2.45 \mathrm{~J} / \mathrm{m}^{2}$ gegenüber Silber mit $\gamma_{\mathrm{Ag}}=1.25 \mathrm{~J} / \mathrm{m}^{2}[\text { Boe } 88]^{13}$ eine deutlich größere spezifische Oberflächenenergie auf und sollte unter dem Aspekt der Minimierung des Oberflächen-zu-Volumen-Verhältnisses präferentiell in den Tälern kompakte Partikel ausbilden. Wie sich das System im Detail verhält, wird wesentlich durch die auftretenden Grenzflächenenergien bestimmt. Nicht mischbare Legierungen zeigen häufig die Tendenz zur Oberflächensegregation der Komponente geringster Oberflächenspannung [Rol85], woraus eine Herabsetzung der freien Oberflächenenergie resultiert. Tober et al. [Tob98] haben hingegen gezeigt, dass die Deposition weniger Monolagen einer CoAgbzw. FeAg-Legierung auf Molybdän zu einer kompositionellen Ordnung führt, wobei sich die Atome unterschiedlicher Spezies unter Ausrichtung an der Kristallstruktur des zugrunde

\footnotetext{
${ }^{13}$ Die Werte wurden anhand entsprechender Daten der flüssigen Phasen und der Temperaturkoeffizienten auf Raumtemperatur extrapoliert und sind daher nur als eine Abschätzung anzusehen.
} 
liegenden Substrats vermutlich infolge einer mechanischen Spannungskompensation in einem lateralen Streifenmuster anordnen.

Aus Ermangelung entsprechender Daten der experimentell schwer zugänglichen temperaturabhängigen Grenzflächenspannungen ist eine genaue Vorhersage darüber, wie sich die Komponenten speziell im Falle niedriger Dimensionalität dünner Schichten unter dem Einfluss der Substratoberfläche verhalten, nicht möglich. Um der räumlichen Verteilung unterschiedlicher Phasen näher auf den Grund zu gehen, werden zum einen die Ergebnisse direkter Untersuchungsmethoden wie die oberflächensensitive XPS-Analyse und TEMQuerschnittsanalysen dargestellt, zum anderen wird die Materialorganisation während thermischer Behandlung kodeponierter NiAg- sowie sukzessiv deponierter Ni- und AgSchichten diskutiert, um ein allgemeineres Verständnis mikrostruktureller Entwicklungen des betreffenden Systems zu ermöglichen und die grundlegenden Prozesse auch in Hinblick auf das anschließende Kapitel der Entnetzung zu analysieren.

\subsection{Oberflächenstruktur kodeponierter NiAg-Schichten}

\subsubsection{Einfluss der kurzwelligen, geordneten Rippelstruktur}

Abbildung 5.1 und Abbildung 5.2 zeigen REM-Aufnahmen einer 50nm dicken gesputterten NiAg-Schicht auf hochgradig geordnetem rippelstrukturierten Substrat (siehe Abschnitt 4.2.2.3) mit einer Wellenlänge von $\lambda=42 \mathrm{~nm}$ sowie auf glattem Substrat. Die Elemente
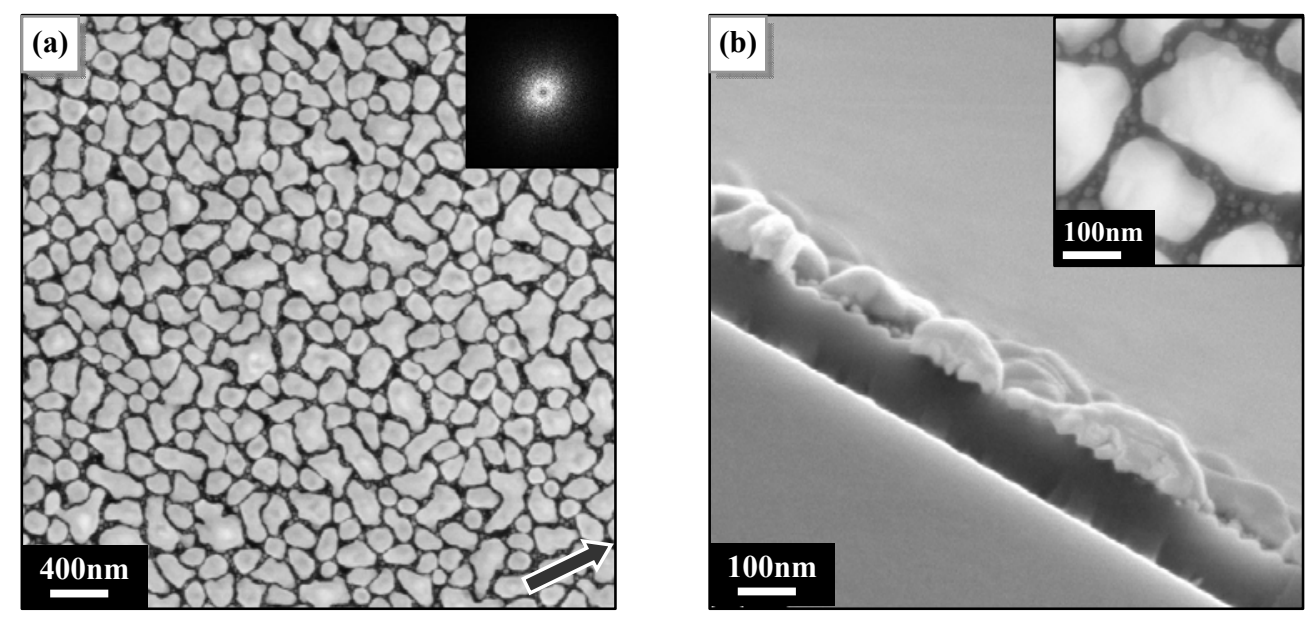

Abbildung 5.1: (a) REM-Aufnahme einer bei $600^{\circ} \mathrm{C}$ gesputterten, $50 \mathrm{~nm}$ dicken NiAg-Schicht auf rippelstrukturiertem Substrat $(\lambda=42 \mathrm{~nm})$ sowie FourierTransformation des Grauwertbildes. Der Pfeil deutet die Richtung des projizierten Ionenstrahls bei der Substratstrukturierung an. (b) Querschnittsaufnahme und vergrößerte Draufsicht derselben Probe. 

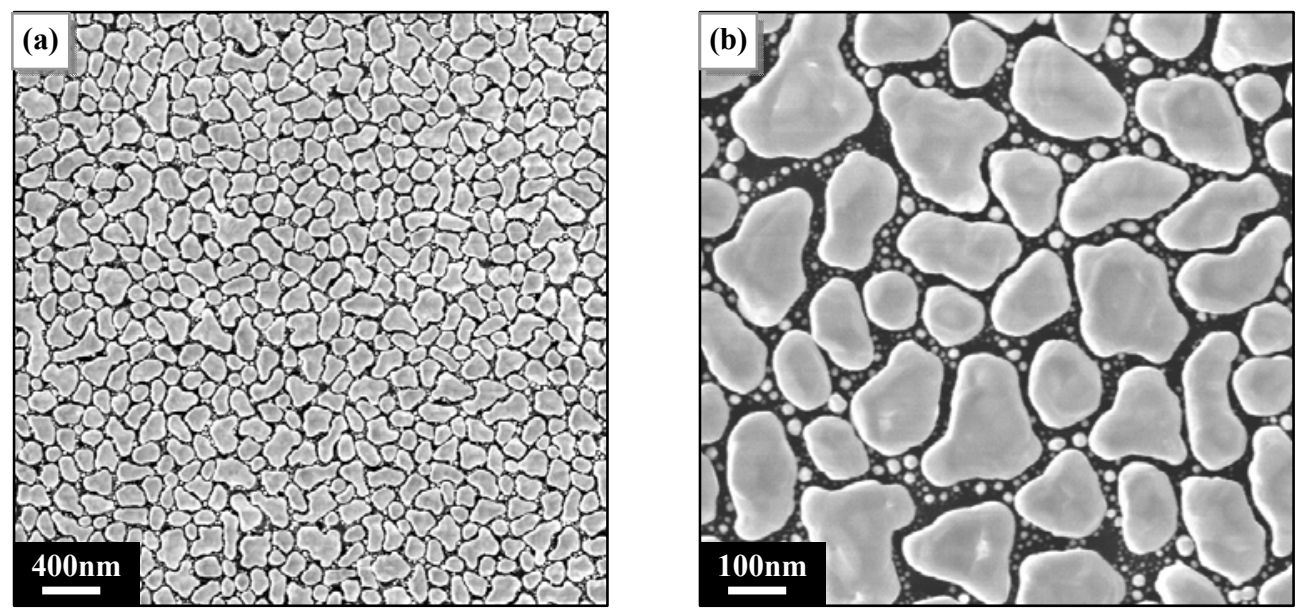

Abbildung 5.2: (a) REM-Aufnahme der bei $600^{\circ} \mathrm{C}$ gesputterten, $50 \mathrm{~nm}$ dicken NiAg-Schicht auf glattem Substrat. (b) Vergrößerter Bildausschnitt.

liegen hierbei in gleichem atomaren Verhältnis vor. Beide Proben weisen ein Gefüge aus isolierten Inseln mit einem mittleren Inseldurchmesser von etwa $170 \mathrm{~nm}$ auf, wobei die große Materialmenge deponierten $\mathrm{NiAg}$ zu einem deutlichen Überwachsen der Rippelstruktur führt (Abbildung 5.1(b)) und sich Inseln bilden, deren Dimension die der zugrunde liegenden Korrugationen deutlich überschreitet. In den tiefen Kanälen zwischen den hochgewachsenen Strukturen sind jedoch auch weitaus kleinere Partikel zu erkennen, deren Größe bis an das Auflösungsvermögen des Rasterelektronenmikroskops $(\sim 5 \mathrm{~nm})$ hinunter reicht. Anordnung und Geometrie der Partikel offenbaren keine Korrelation mit der Wellenstruktur der Grenzfläche zum Substrat.

Unter Verwendung eines Schwellenwertes bezüglich der Grauwertskala der REMAufnahmen wurden die Inseln vom Untergrund separiert und ein Histogramm der flächenäquivalenten Inseldurchmesser errechnet (Abbildung 5.3). Die Kurven zeigen eine nahezu identische Verteilung der Inselgrößen auf glattem und strukturiertem Substrat mit einem

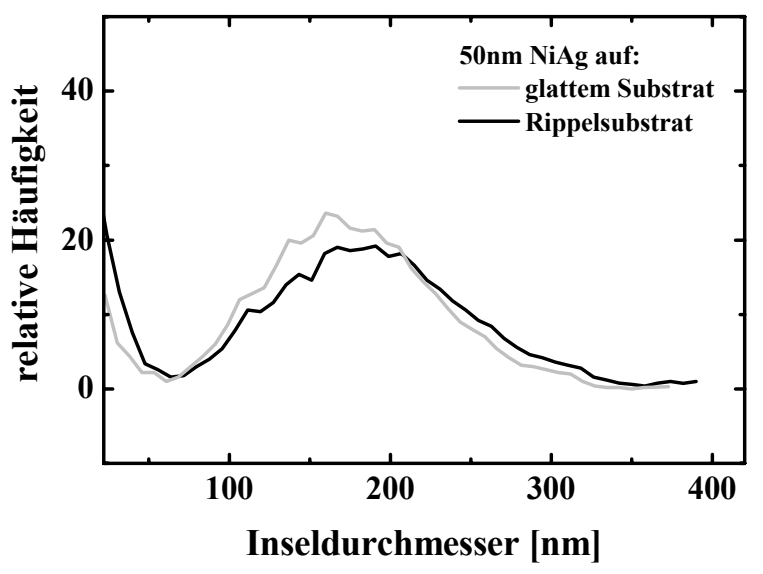

Abbildung 5.3: Verteilung der Inseldurchmesser. 
breiten Maximum um etwa 170nm sowie einem Minimum bei etwa $60 \mathrm{~nm}$. $\mathrm{Zu}$ sehr kleinen Durchmessern hin verliert die Verteilung methodisch bedingt auf Grund der begrenzten Auflösung des Rasterelektronenmikroskops und des jeweiligen Bildausschnitts sowie Randeffekten der kontrastschwächeren kleinen Inseln bei der Schwellenwertbestimmung ihre Aussagekraft, weshalb die Graphen an der betreffenden Stelle abgeschnitten wurden. Ein Messartefakt konnte als Ursache der bimodalen Verteilung durch die Verifizierung des Auswertungsverfahrens anhand von Bildausschnitten unterschiedlicher Auflösung sowie der systematischen Verschiebung des Minimums zu größeren Durchmessern unabhängig von der Größe und Auflösung des Bildausschnitts bei Proben mit Schichtdicken zwischen $5 \mathrm{~nm}$ und $300 \mathrm{~nm}$ ausgeschlossen werden. Die leicht erhöhte relative Häufigkeit von Inseldurchmessern unterhalb des Minimums deutet möglicherweise auf eine stärkere Koaleszenz von Partikeln $<60 \mathrm{~nm}$ auf der strukturierten Oberfläche.

Mit der Reduzierung der deponierten Materialmenge entsprechend einer Schichtdicke von $20 \mathrm{~nm}$ und der damit verbundenen Senkung mittlerer Inselgröße entsteht eine deutlich veränderte Oberflächenstruktur. Abbildung 5.4 zeigt REM-Aufnahmen der präparierten Schicht auf rippelstrukturiertem Substrat. Die Inseln sind in linearen Ketten mit dazu senkrechter periodischer Korrelation angeordnet, wobei die Analyse der Fourier-Transformation eine Übereinstimmung der Korrelationslänge mit dem Abstand der Rippel ergibt. Der Vergleich zu der auf glattem Substrat deponierten Schicht (Abbildung 5.5(a)) verdeutlicht, dass Form und Größe der Strukturen ebenfalls einen signifikanten Einfluss der zugrunde liegenden Grenzflächenmorphologie aufweisen. Die Inseln lassen auf dem Template überwiegend eine nahezu kreisförmige Geometrie erkennen, wobei nur wenige dieser Partikel zu länglichen Strukturen mit paralleler Ausrichtung zur Rippelorientierung zusammengewachsen sind. Die Fourier-Transformation des vergrößerten Ausschnitts (Abbildung 5.4(b)) offen-
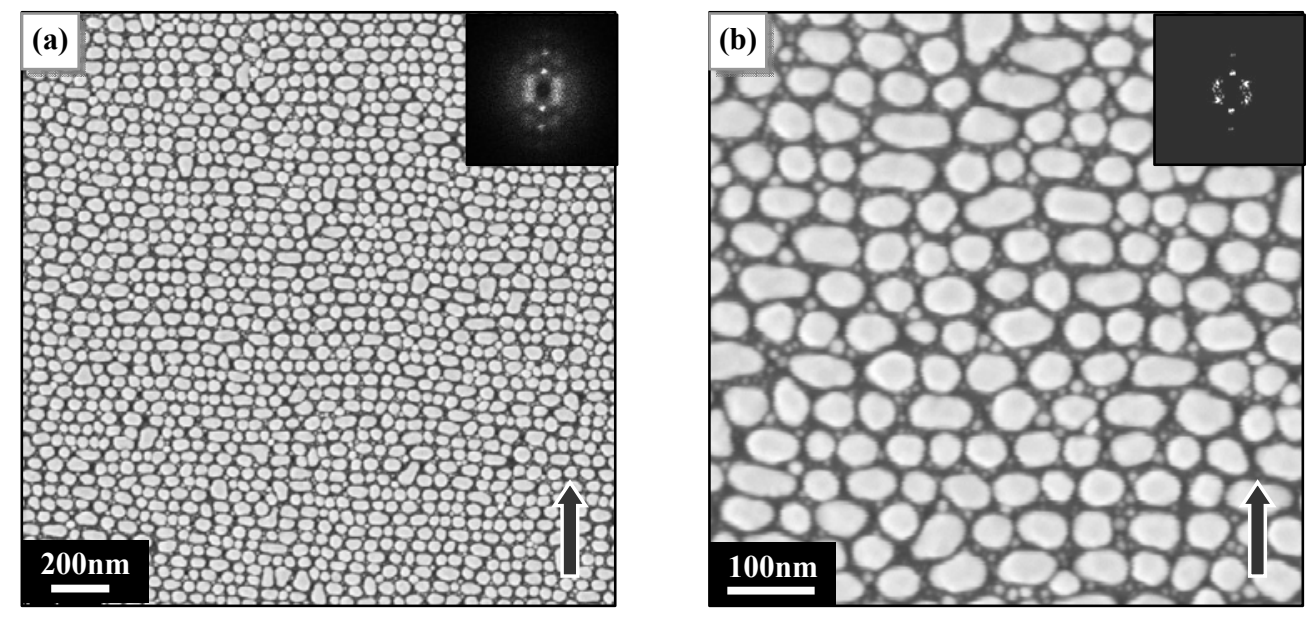

Abbildung 5.4: (a) REM-Aufnahme einer bei $600^{\circ} \mathrm{C}$ gesputterten, $20 \mathrm{~nm}$ dicken NiAg-Schicht auf rippelstrukturiertem Substrat $(\lambda=42 \mathrm{~nm})$ sowie FourierTransformation des Grauwertbildes. (b) Vergrößerter Bildausschnitt. 

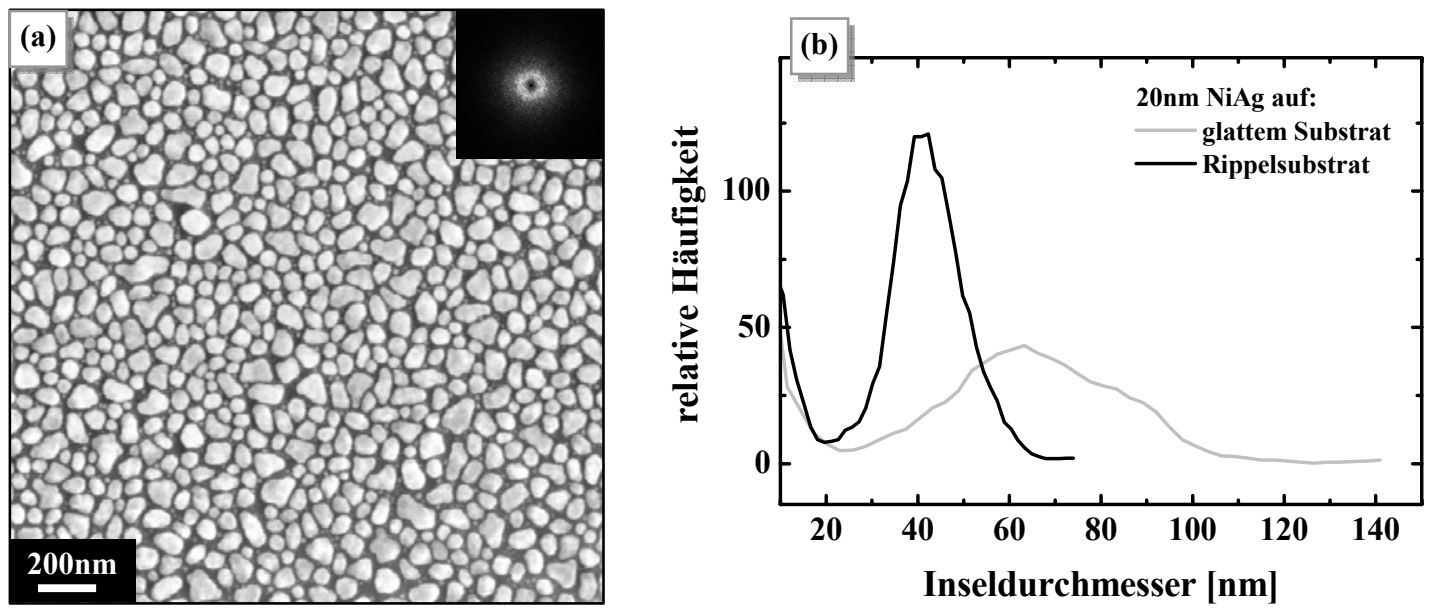

Abbildung 5.5: (a) REM-Aufnahme der bei $600^{\circ} \mathrm{C}$ gesputterten, 20nm dicken NiAg-Schicht auf glattem Substrat. (b) Verteilung der Inseldurchmesser.

bart eine hexagonale Nahordnung, die allgemein eine Anordnung mit maximaler Packungsdichte erlaubt. Eine Auswertung mittels der Autokorrelationsfunktion ergibt einen Partikelabstand entlang der Täler von $X_{M, P}=53 \mathrm{~nm}$ sowie $X_{M, S}=42 \mathrm{~nm}$ senkrecht hierzu und offenbart eine geringe Asymmetrie der hexagonalen Struktur.

In Abbildung 5.5(b) ist die Häufigkeitsverteilung der Inseldurchmesser beider 20nm dicken NiAg-Schichten graphisch aufgetragen. Auf glattem Substrat zeigt sich ein breites Maximum um $65 \mathrm{~nm}$ sowie ein Minimum bei $24 \mathrm{~nm}$. Auf rippelstrukturiertem Substrat ist die Verteilung zu weitaus kleineren Inselgrößen verschoben, was sich auch direkt aus den entsprechenden REM-Aufnahmen (Abbildung 5.4(a) und Abbildung 5.5(a)) erschließt. Die modulierte Grenzfläche induziert dabei ein deutlich schärferes Maximum um 42nm, womit der am häufigsten auftretende Partikeldurchmesser bei geringer Streuung exakt der Wellenlänge dieser Modulation entspricht. Das Minimum der Verteilung verschiebt sich nur leicht auf 20nm. Auch hier weisen die Zwischenräume zwischen relativ großen Inseln deutlich kleinere Partikel auf, die scheinbar regellos angeordnet sind und keine Beeinflussung durch die Oberflächenstruktur des Substrats erkennen lassen (Abbildung 5.4(b)), sodass die Verteilung der Inseldurchmesser für Größen unterhalb des Minimums etwa der auf glattem Substrat entspricht.

\subsubsection{Einfluss der tiefen, unregelmäßigen Rippelstruktur}

Zur Untersuchung des Einflusses einer Oberflächentopographie mit unterschiedlicher Wellenlänge und Tiefe der Rippelstrukturen auf das Schichtwachstum wurden NiAg-Schichten einer Dicke von 20nm (Abbildung 5.7) bzw. 50nm (Abbildung 5.6) auf entsprechend gröber strukturierten Substraten (siehe Abschnitt 4.2.2.4) deponiert. Diese besitzen ein Spek- 

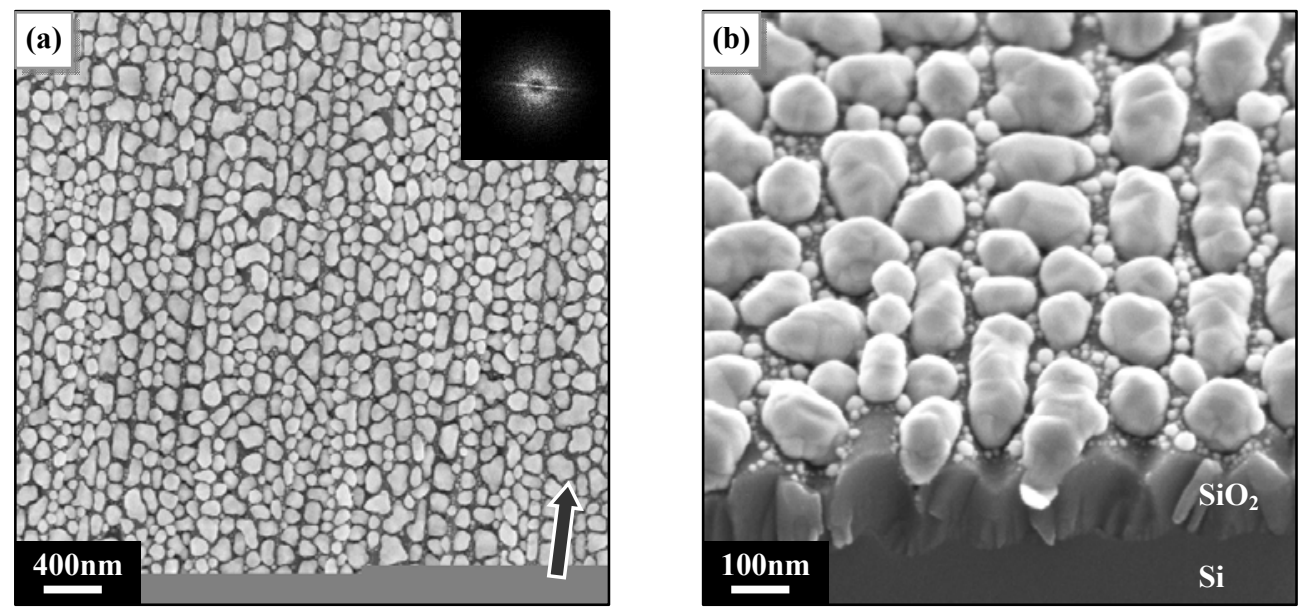

Abbildung 5.6: (a) REM-Aufnahme einer bei $600^{\circ} \mathrm{C}$ gesputterten, 50nm dicken NiAg-Schicht auf ionenstrahlstrukturiertem Substrat. (b) REM-Aufnahme bei einer Verkippung von $40^{\circ}$ an einer Bruchkante, die nach der Deposition erzeugt wurde.
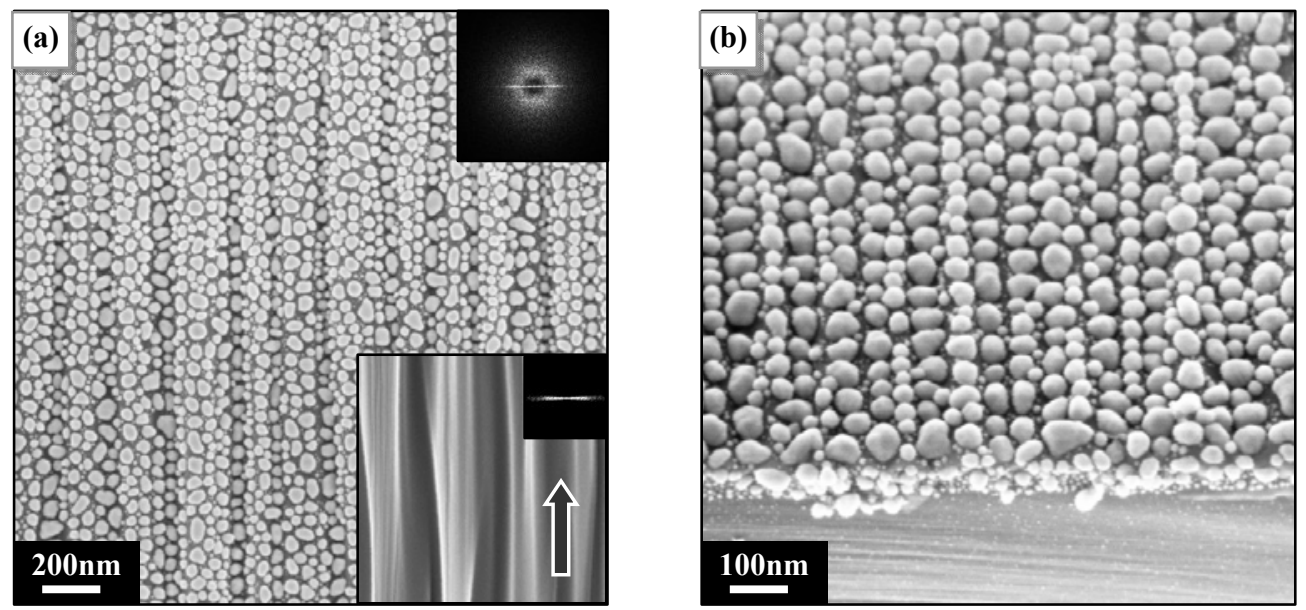

Abbildung 5.7: (a) REM-Aufnahme einer bei $600^{\circ} \mathrm{C}$ gesputterten, $20 \mathrm{~nm}$ dicken NiAg-Schicht sowie Fourier-Transformation des Grauwertbildes und REMAufnahme der ionenstrahlstrukturierten Substratoberfläche vor der Deposition mit identischer Vergrößerung. (b) REM-Aufnahme bei einer Verkippung von $45^{\circ}$ an einer Bruchkante, die vor der Deposition erzeugt wurde.

trum an unregelmäßigen Korrugationen mit einer lateralen Größe von bis zu 400nm sowie kleinere überlagerte Störungen bis hin zu einigen 10nm Wellenlänge.

Beide Proben weisen, induziert durch die Grenzflächenkorrugationen, eine verstärkt kettenartige Anordnung der Inseln auf. Im Gegensatz zur geordneten Rippelstruktur zeigt die jeweilige Fourier-Transformation aus Ermangelung einer besonders signifikanten Modulation der Substratoberfläche auch nach Schichtdeposition keine hervortretende unidirektionale Periodizität. 

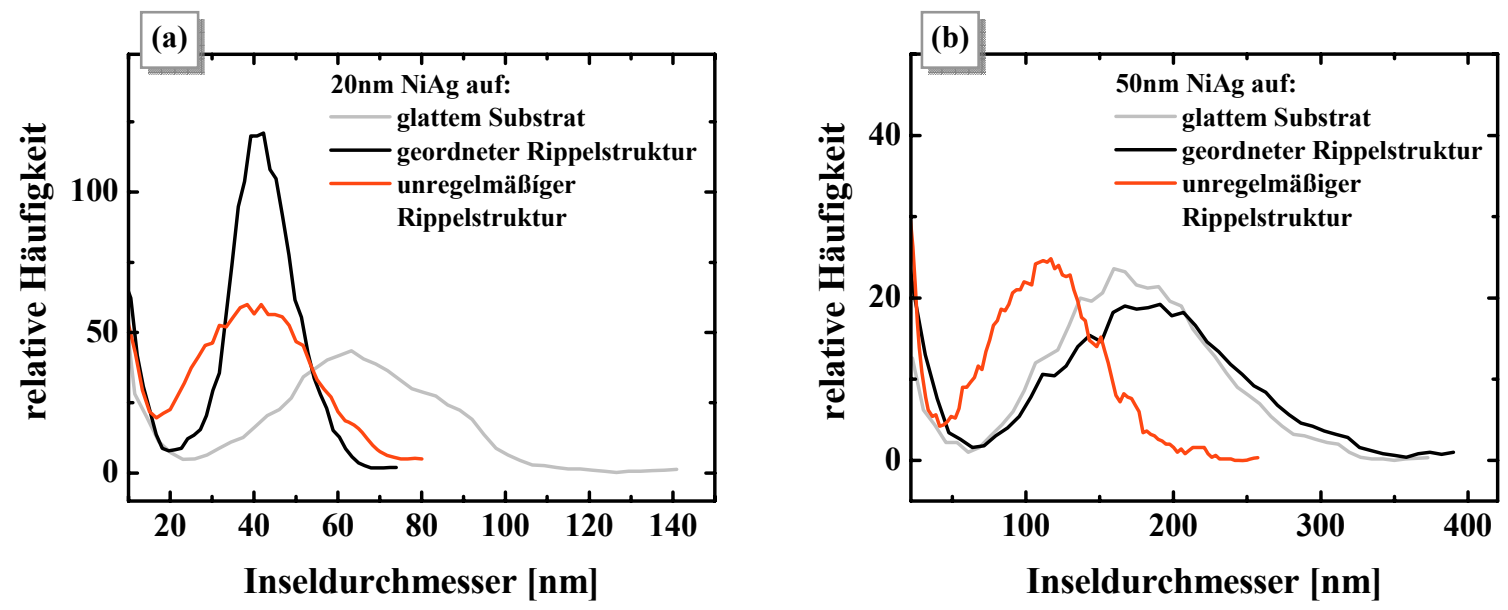

Abbildung 5.8: Verteilung der Inseldurchmesser bei (a) 20nm bzw. (b) $50 \mathrm{~nm}$ Schichtdicke auf den unterschiedlichen Substraten.

In Abbildung 5.8 sind die ermittelten Inseldurchmesserverteilungen dargestellt. Bei 20nm Schichtdicke wird eine im Vergleich zu dem Verhalten auf der geordneten Rippelstruktur ähnliche Verschiebung der mittleren Inselgröße zu 40nm beobachtet, wobei das Maximum jedoch merklich verbreitert ist. Im Gegensatz zur kurzwelligen Rippelstruktur induziert die viele unterschiedliche Periodizitäten enthaltende Grenzfläche selbst bei $50 \mathrm{~nm}$ Schichtdicke eine deutliche Reduktion der Inselgrößen. Das Maximum liegt indes mit $115 \mathrm{~nm}$ bei größeren Durchmessern, wodurch die Modulationen, die die Inselgröße bei $20 \mathrm{~nm}$ Schichtdicke maßgeblich beeinflussen, überwachsen werden. Dies zeigt, dass die kettenartige Anordnung auch während des Zusammenwachsens benachbarter Inseln im Wesentlichen erhalten bleibt, solange die Materialmenge die Dimension der Rippelstruktur nicht bei Weitem übersteigt, und eine Anpassung der Schichtdicke zur Rippelwellenlänge erforderlich ist, um eine scharfe Größenverteilung hervorzurufen.

Die Aufnahme an der Bruchkante der 50nm Probe verdeutlicht, dass sich die Inseln vermehrt in den Tälern sammeln, wobei die signifikant kleineren Inseln zwischen den hochgewachsenen Partikeln keine besondere Anordnung erkennen lassen (Abbildung 5.6(b)) und scheinbar auch durch die kleineren Korrugationen nicht beeinflusst werden. Dieser Eindruck wird durch die nahezu identische Größenverteilung geringerer Inseldurchmesser in Bezug auf die entsprechende Schicht auf glattem Substrat bekräftigt.

\subsection{Magnetisierungsverhalten}

Zur Überprüfung der magnetischen Eigenschaften der aus im Allgemeinen ferromagnetisch ordnendem Nickel und diamagnetischem Silber zusammengesetzten Schichten speziell in Hinblick auf eine mögliche Induzierung einer Anisotropie wurden Magnetisierungskurven der 20nm NiAg-Schicht unter Ausnutzung des magnetooptischen Kerreffekts aufgezeich- 




Abbildung 5.9: Magnetisierungsverhalten der bei $600^{\circ} \mathrm{C}$ auf geordneter Rippelstruktur deponierten 20nm NiAg-Schicht mit auf die Sättigungsmagnetisierung normierter Magnetisierung $\mathrm{M} / \mathrm{M}_{\mathrm{S}}$.

net. Hierbei wurde sowohl mit paralleler als auch mit senkrechter Ausrichtung des äußeren Magnetfeldes zu der durch Ionenbestrahlung hervorgerufenen geordneten Rippelstruktur der Substratoberfläche gemessen.

Die Probe zeigt ein typisch ferromagnetisches Hystereseverhalten ohne merkliche Anisotropie mit im Rahmen der Streuung übereinstimmender reduzierter Remanenz $\mathrm{M}_{\mathrm{R}} / \mathrm{M}_{\mathrm{S}}=$ 0.75 sowie identischem Koerzitiv- und Sättigungsfeld $\mathrm{H}_{\mathrm{C}}=200 \mathrm{Oe}$ bzw. $\mathrm{H}_{\mathrm{S}} \approx 900 \mathrm{Oe}$ (Abbildung 5.9). Die spezielle regelmäßige Anordnung magnetischer Nanopartikel lässt keine signifikanten Auswirkungen auf das Magnetisierungsverhalten erkennen.

\subsection{Elementspezifische Verteilung}

Um die chemische Zusammensetzung der Oberfläche und tiefer liegender Lagen zu bestimmen, wurde eine 50nm NiAg-Schicht auf glattem Substrat mittels XPS-Analyse untersucht und dessen Oberfläche durch einen $2.3 \mathrm{keV} \mathrm{Ar}^{+}$-Ionenstrahl unter einem Winkel von $60^{\circ}$ sukzessiv abgetragen. Die XPS-Spektren vor der Sputtererosion und nach einer Erosionszeit von insgesamt 100 Minuten sind in Abbildung 5.10(a) dargestellt. Hierbei zeigt sich eine deutliche Zunahme des Nickelsignals und eine entsprechende Abnahme des Silbersignals durch den Erosionsvorgang. Zwecks relativer Konzentrationsbestimmung wurden jeweils die Ni-2p- und Ag-3d-Kanten herangezogen und das infolge der teilweise freiliegenden Substratoberfläche zusätzlich auftretende $\mathrm{SiO}_{2}$-Signal vernachlässigt. Nach Schichtdeposition weist die Probe eine relative Silberkonzentration von nahezu 100\% auf, die sich im Zuge des Oberflächenabtrags nach 100 Minuten auf etwa 10\% reduziert (Abbildung 5.10(b)). Dabei ist kritisch anzumerken, dass die Genauigkeit der quantitativen Auswertung auf Grund apparativer Faktoren hier nicht bekannt ist (siehe Abschnitt 2.8) und genannte Absolutwerte somit eher qualitative Aussagekraft besitzen. Ein Elementnachweis 

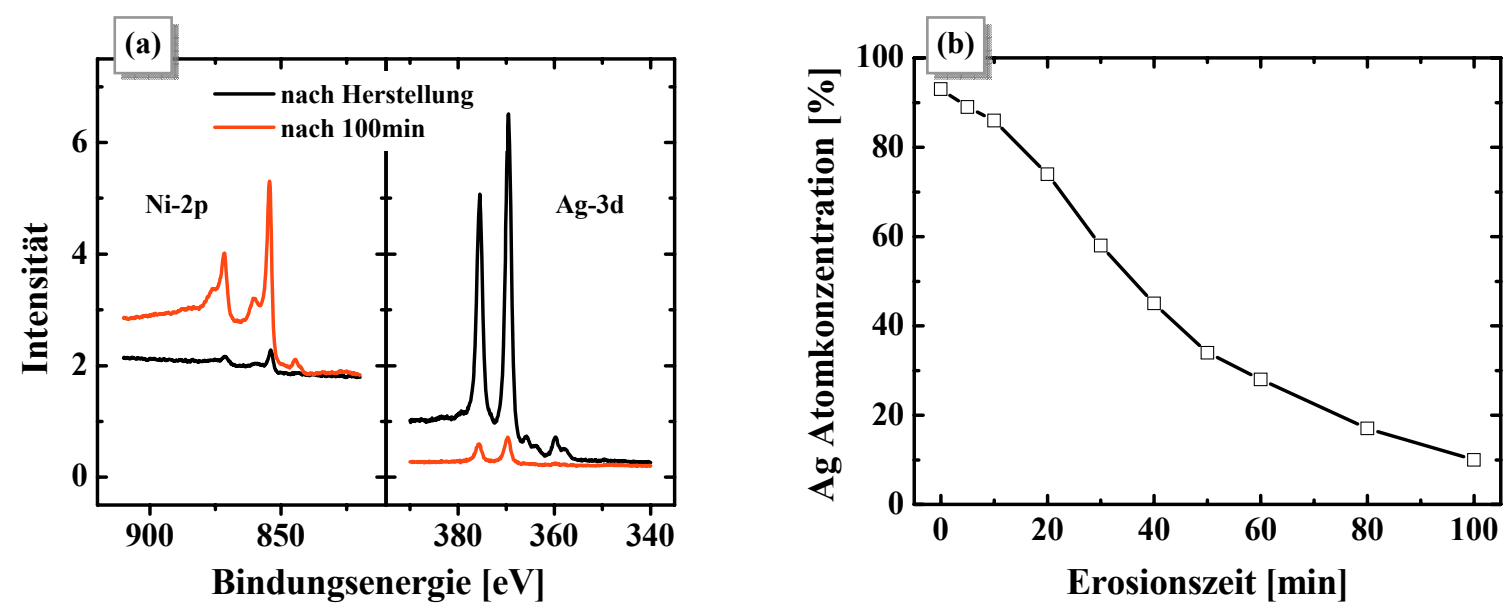

Abbildung 5.10: (a) XPS-Spektren vor der Sputtererosion einer 50nm NiAgSchicht auf glattem Substrat sowie nach 100min Erosionszeit. (b) Entwicklung der Ag-Oberflächenkonzentration während sukzessiver Sputtererosion.
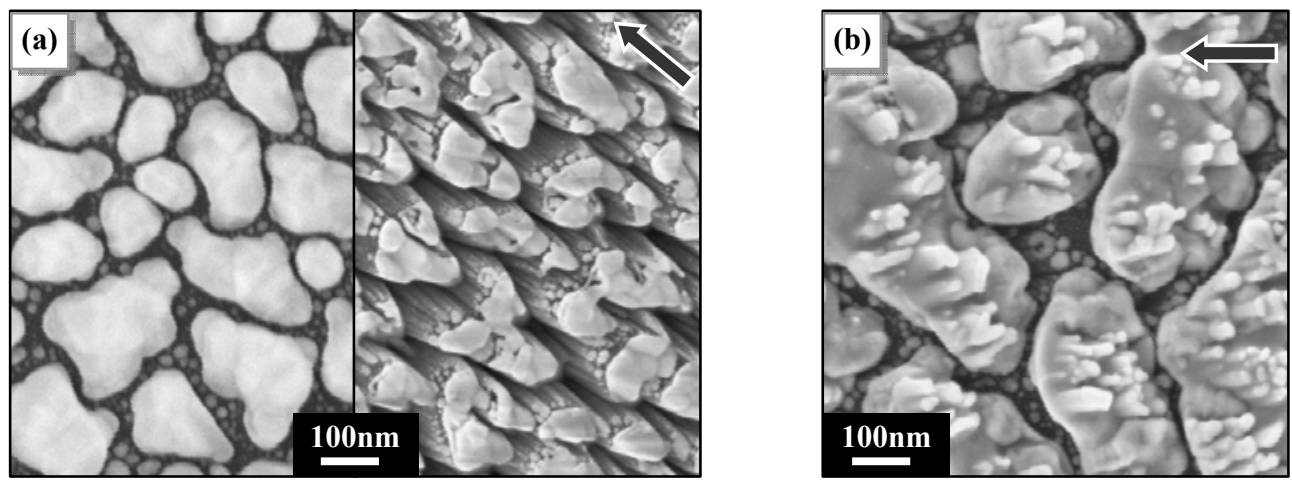

Abbildung 5.11: (a) REM-Aufnahme der 50nm NiAg-Schicht vor und nach 100min Sputtererosion. (b) REM-Aufnahme einer 100nm NiAg-Schicht nach 60min Erosionsdauer.

ist hingegen selbst für Konzentrationen $<1 \%$ möglich. Ferner bewirkt der Sputterprozess nicht allein den Abtrag der Oberfläche, sondern verursacht mikrostrukturelle Veränderungen (siehe Kapitel 3), die sich jedoch auf Grund der vergleichsweise geringen Ionenenergie und -reichweite $(\sim 2 \mathrm{~nm}$ nach Berechnungen mittels SRIM) auf eine dünne oberflächennahe Schicht beschränken.

Abbildung 5.11(a) zeigt REM-Aufnahmen vor und nach der Sputtererosion der oben behandelten Probe. Es entstehen säulenartige Strukturen mit Schluchten, die tief in das Substrat hineinreichen. Die Spitzen der Säulen sind jeweils mit einer dünnen Metallschicht bedeckt. Abbildung 5.11(b) zeigt die Oberflächenentwicklung einer 100nm NiAg-Schicht nach einer Erosionsdauer von 60 Minuten und verdeutlicht, dass der Erosionsprozess auf Grund von Einflüssen wie dem unterschiedlichen Sputter-Yield entlang unterschiedlicher 

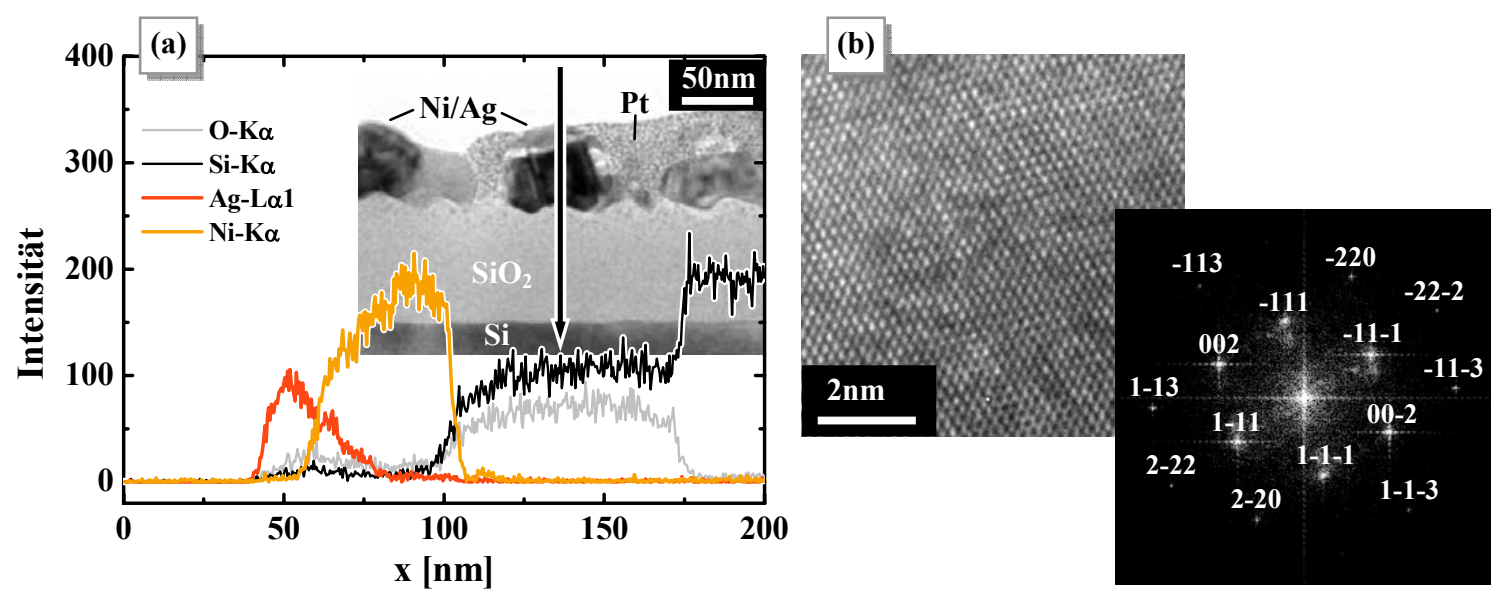

Abbildung 5.12: (a) Hellfeld-TEM-Aufnahme einer 50nm NiAg-Schicht auf geordnetem Rippelsubstrat sowie EDX-Linienprofil entlang des Pfeils. (b) Hochaufgelöste TEM-Aufnahme des Ni mit indizierter Fourier-Transformation.
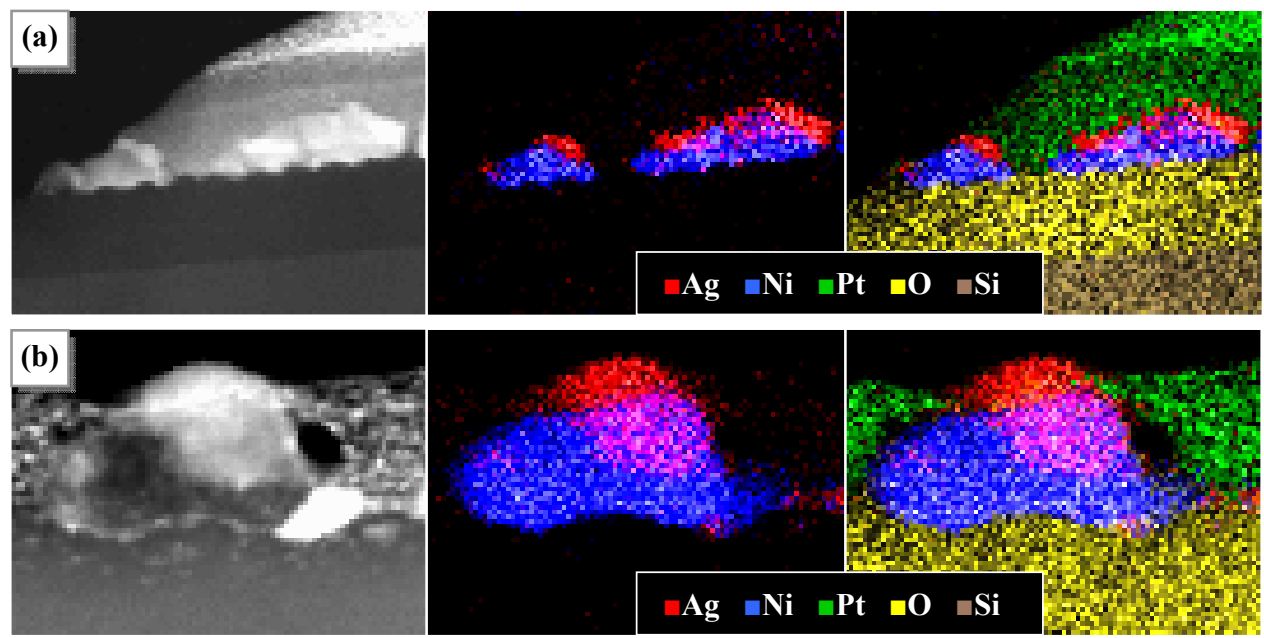

Abbildung 5.13: (a) und (b) zeigen jeweils eine Dunkelfeld-TEM-Aufnahme und zweidimensionale elementaufgelöste EDX-Kartierungen, wobei in der mittleren Abbildung nur die Elemente Ni und Ag verzeichnet sind.

kristallographischer Richtungen und verschiedener Materialien sowie durch Abschattungseffekte nicht zu einem gleichmäßigen schichtweisen Abtrag führt, sodass streng genommen kein reines Tiefenprofil aufgezeichnet werden kann. Die Untersuchungen erlauben indes die Schlussfolgerung einer deutlichen Oberflächensegregation des Silbers und deuten auf eine Abnahme der Silberkonzentration in tieferen Lagen.

Die EDX-Analyse im Transmissionselektronenmikroskop an einer entsprechend dünn präparierten Querschnittslamelle ermöglicht genauere ortsaufgelöste Untersuchungen. In Abbildung 5.12(a) ist eine TEM-Querschnittsaufnahme einer 50nm NiAg-Schicht auf ge- 
ordnetem Rippelsubstrat sowie ein EDX-Linienprofil dargestellt. Hieran wird deutlich, dass sich das Silber nahezu vollständig an der Oberfläche der betrachteten Insel ansammelt, während sich das Silbersignal an der Grenzfläche zum Substrat nicht vom Untergrundrauschen abhebt. Anhand der Fourier-Transformation einer hochaufgelösten TEM-Aufnahme (Abbildung 5.12(b)) mit entsprechender Indizierung durch Verwendung des Gitterparameters von Nickel und einen Vergleich mit indizierten Beugungsbildern kubischflächenzentrierter Einkristalle [Ful02] ergibt sich, dass Nickel auch auf der Rippelstruktur in $<111>$ Richtung aufwächst.

Eine elementspezifische Analyse kleinerer Partikel mit geringem Abstand, wie sie bei der 20nm dicken NiAg-Schicht auf der geordneten Rippelstruktur zu beobachten sind, ist im TEM auf Grund der Lamellenbreite, die sich in der Größenordnung von 100nm bewegt, nicht ohne Weiteres zugänglich, da hier mehrere Inseln durchstrahlt werden, wodurch eine eindeutige Zuordnung der Signale zu den einzelnen Partikeln nicht möglich ist. Bei TEMUntersuchungen ist allgemein zu beachten, dass auf Grund des Präparationsverfahrens zur Herstellung elektronentransparenter Lamellen mittels Ionenstrahlverfahren oberflächliche mikrostrukturelle Veränderungen durch strahlungsinduzierte Effekte nicht vollständig zu eliminieren sind. Eine strahlungsinduzierte Entmischung der Inseln ist jedoch trotz der hier teilweise zu weit erodierten Platinschutzschicht in Anbetracht der mittleren Ioneneindringtiefe des $30 \mathrm{keV} \mathrm{Ga}^{+}$-Strahls von 10nm (bestimmt mittels SRIM) auszuschließen.

Zweidimensionale elementspezifische EDX-Kartierungen (Abbildung 5.13) bestätigen die Segregation des Silbers an die Oberfläche. Isolierte Silberausscheidungen sind im Nickel nicht zu identifizieren und eine laterale Trennung der Phasen wird ebenfalls nicht beobachtet. Das Silber ist offensichtlich bestrebt, eine Grenzfläche mit der Substratoberfläche zu vermeiden.

\subsection{Strukturentwicklung in Ni-Ag-Schichten während ther- mischer Behandlung}

In diesem Abschnitt soll der Fragestellung nachgegangen werden, wie sich eine thermische Behandlung auf die mikrostrukturelle Entwicklung bei Raumtemperatur kodeponierter sowie sukzessiv deponierter Ni-Ag-Schichten auswirkt, um mittels einer Vergleichsstudie die vorherrschenden Prozesse der Organisation beider Phasen zu identifizieren.

Kodeponierte NiAg-Schichten: Hierzu wurde zunächst bei Raumtemperatur eine mittels Sputterdeposition kodeponierte, 10nm dicke NiAg-Schicht auf thermisch oxidiertem Silizium hergestellt und anschließend in situ bei $600^{\circ} \mathrm{C}$ für eine Stunde ausgelagert. Nach Deposition zeigt sich eine vergleichsweise glatte Oberfläche, deren feine Struktur im REM nur schwerlich aufzulösen ist (Abbildung 5.14(a)). Vereinzelt heben sich etwa 5-10nm gro- 

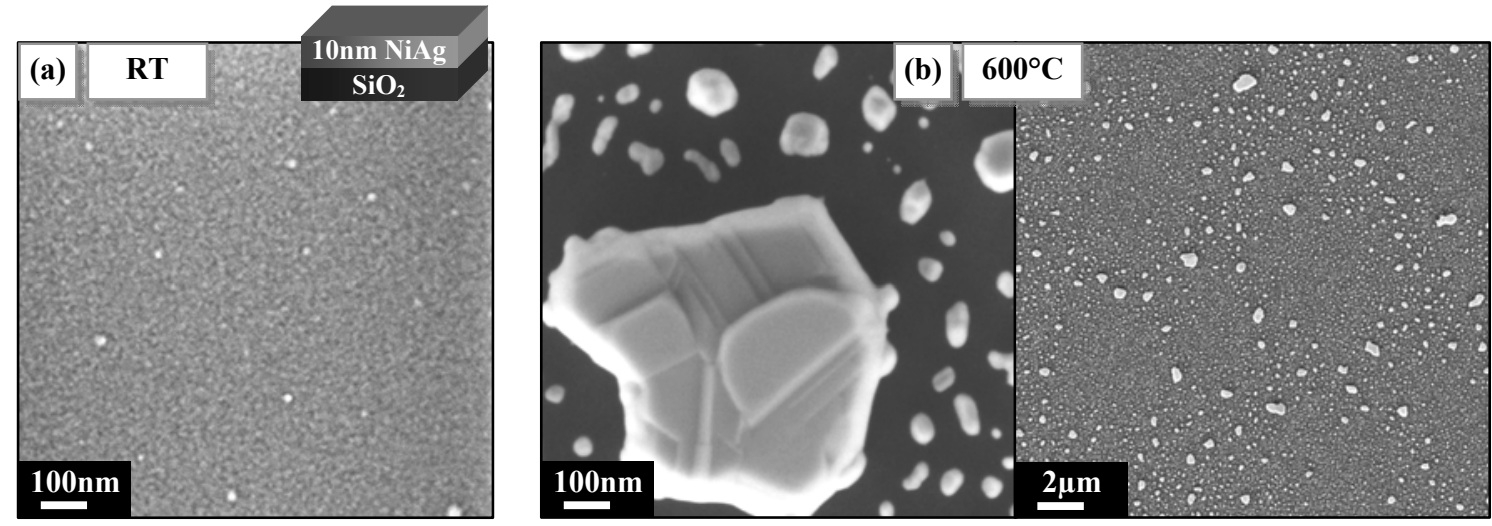

Abbildung 5.14: REM-Aufnahmen einer (a) bei Raumtemperatur kodeponierten und anschließend (b) für $1 \mathrm{~h}$ bei $600^{\circ} \mathrm{C}$ thermisch behandelten $10 \mathrm{~nm} \mathrm{NiAg-}$ Schicht.

ße Körner vom Untergrund ab. Die XPS-Analyse liefert eine Oberflächenkonzentration von $62 \% \mathrm{Ag}$ und $38 \% \mathrm{Ni}$, was möglicherweise bereits eine leichte Oberflächensegregation des Silbers offenbart.

Nach thermischer Behandlung zeigt sich - auch im Vergleich zu der bei $600^{\circ} \mathrm{C}$ kodeponierten NiAg-Schicht gleicher Materialmenge (siehe Anhang C) - ein deutlich verändertes Bild (Abbildung 5.14(b)). Die ursprünglich zusammenhängende Schicht reißt auf und durch den Agglomerationsprozess entsteht ein Gefüge aus kleinen Inseln mit einer mittleren Größe von etwa 50nm, die sich weitestgehend homogen über die Oberfläche verteilen, und netzartig angeordneten Partikeln mit einem Durchmesser von bis zu 600nm. Eine EDX-Analyse der großen Partikel ergibt einen Silberanteil von nahezu 95\%, was nahelegt, dass die vielen kleineren Inseln im Wesentlichen aus Nickel bestehen, während Silberatome eine deutlich höhere Mobilität besitzen und große Inseln in einem Abstand von einigen 100nm bilden. Die Tatsache, dass die EDX-Spektren ein Nickelsignal aufweisen, welches sich klar vom Untergrundrauschen abhebt, deutet auf darunter liegende Nickelpartikel, die vereinzelt am Rand der großen Inseln herausragen (Abbildung 5.14(b)). Eine entsprechende Analyse der kleinen Inseln ist zum einen auf Grund der geringen Materialmenge, die eine quantitative Bestimmung der Zusammensetzung verhindert (siehe Abschnitt 2.7), nicht möglich. Zum anderen konnte hier der Elektronenstrahl durch Drift vermutlich infolge von Aufladungseffekten nicht ausreichend lange in Position gehalten werden, um ein eventuelles Silbersignal nachzuweisen oder auszuschließen. Die XPS-Analyse liefert indes eine relative Oberflächenzusammensetzung von 85\% Ag und 15\% Ni nach Temperaturbehandlung und indiziert in Anbetracht des hohen Oberflächenanteils der kleineren Inseln somit, dass ein Teil der kleineren Partikel ebenfalls Silber enthält.

Die thermische Behandlung reiner Ni- bzw. Ag-Schichten zeigt, dass das beobachtete Gefüge der wärmebehandelten NiAg-Schicht nicht durch unabhängige Agglomeration der beiden Phasen auf der Substratoberfläche zustande kommt und die Mikrostruktur eine 

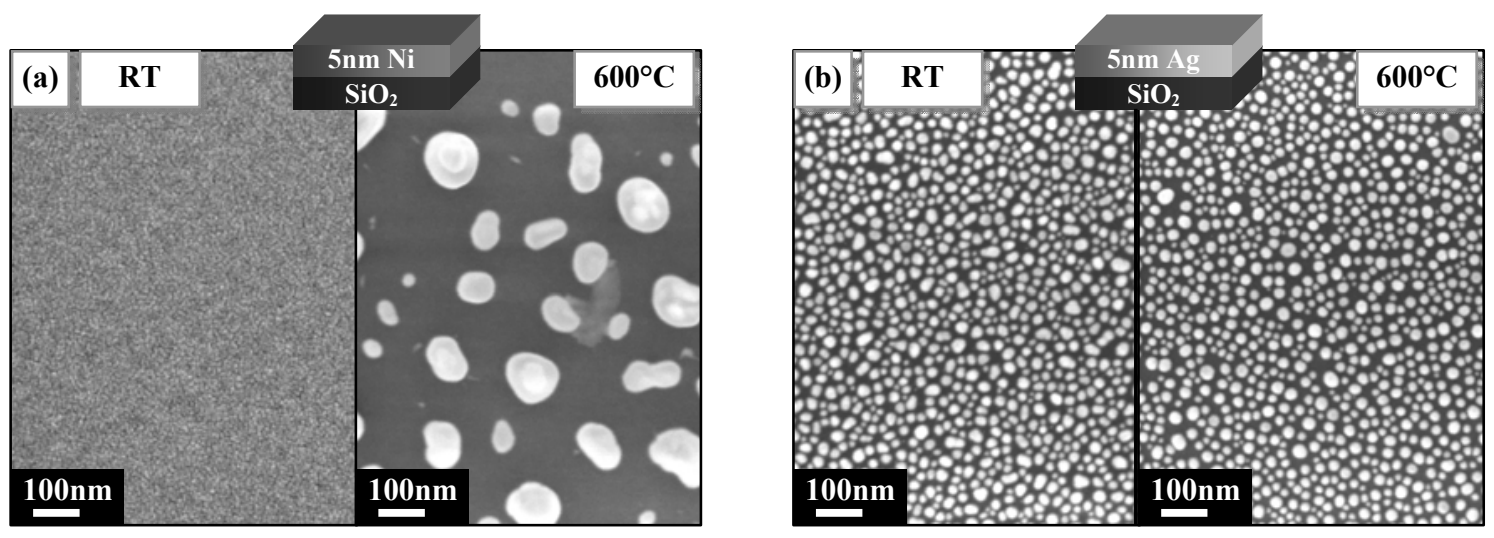

Abbildung 5.15: REM-Aufnahmen einer (a) bei Raumtemperatur deponierten und anschließend für $1 \mathrm{~h}$ bei $600^{\circ} \mathrm{C}$ thermisch behandelten $5 \mathrm{~nm}$ Ni-Schicht vor und nach der Temperaturbehandlung sowie (b) einer entsprechend behandelten $5 \mathrm{~nm}$ Ag-Schicht.

Überlagerung der reinen, getemperten Schichten ist, sondern gekoppelte Prozesse stattfinden. Eine bei Raumtemperatur aufgedampfte $5 \mathrm{~nm}$ Nickelschicht bildet zunächst einen zusammenhängenden Film, der bei hoher Temperatur in bis zu 100nm große isolierte Inseln umgewandelt wird (Abbildung 5.15(a)). Silber weist schon nach Deposition dicht gepackte isolierte Inseln einer maximalen Größe von ca. 20nm auf und zeigt keinen merklichen Einfluss der Temperaturbehandlung auf die Mikrostruktur (Abbildung 5.15(b)). Folglich entstehen die großen, silberreichen Partikel erst in Verbindung mit Nickel.

Silber auf Nickel: Welche Bedeutung das Nickel dabei besitzt, wird an folgender Untersuchung deutlich. Mittels Elektronenstrahlverdampfens wurden 5nm-Ag/5nm-Ni-Doppellagen bei Raumtemperatur deponiert und anschließend in situ bei unterschiedlichen Temperaturen für eine Stunde ausgelagert (Abbildung 5.16). Hierbei zeigt sich der Einfluss des zugrunde liegenden Substratmaterials auf das Wachstumsverhalten dünner Schichten. Silber bildet auf Nickel im Gegensatz zum $\mathrm{SiO}_{2}$-Substrat bei Raumtemperatur zunächst eine glatte zusammenhängende Schicht mit kleinen Löchern (Abbildung 5.16(a)). Eine wesentliche Rolle kommt dabei den Bindungsverhältnissen zu, die das Diffusionsverhalten während des Wachstums direkt beeinflussen und die Grenzflächenenergie bestimmen. Zudem besitzen Metalle im Allgemeinen eine höhere Oberflächenenergie als dielektrische Materialien $\left(\gamma_{\mathrm{SiO} 2}(1573 \mathrm{~K})=307 \mathrm{~mJ} / \mathrm{m}^{2}\right.$ [Han75], $\left.\gamma_{\mathrm{Ni}}(1673 \mathrm{~K})=1940 \mathrm{~mJ} / \mathrm{m}^{2}[\mathrm{Kum} 83]\right)$.

Während einstündiger Temperaturbehandlung von $200^{\circ} \mathrm{C}$ breiten sich die Löcher aus und es entstehen kompakte Partikel mit etwa 200nm Durchmesser in einem Abstand von mehreren Mikrometern (Abbildung 5.16(c)). EDX-Linienprofile entlang der Oberfläche zeigen ein konstantes Nickelsignal bei in den Löchern abfallendem sowie auf den Partikeln ansteigendem Silbersignal (Abbildung 5.17) und belegen somit, dass es sich hierbei um 

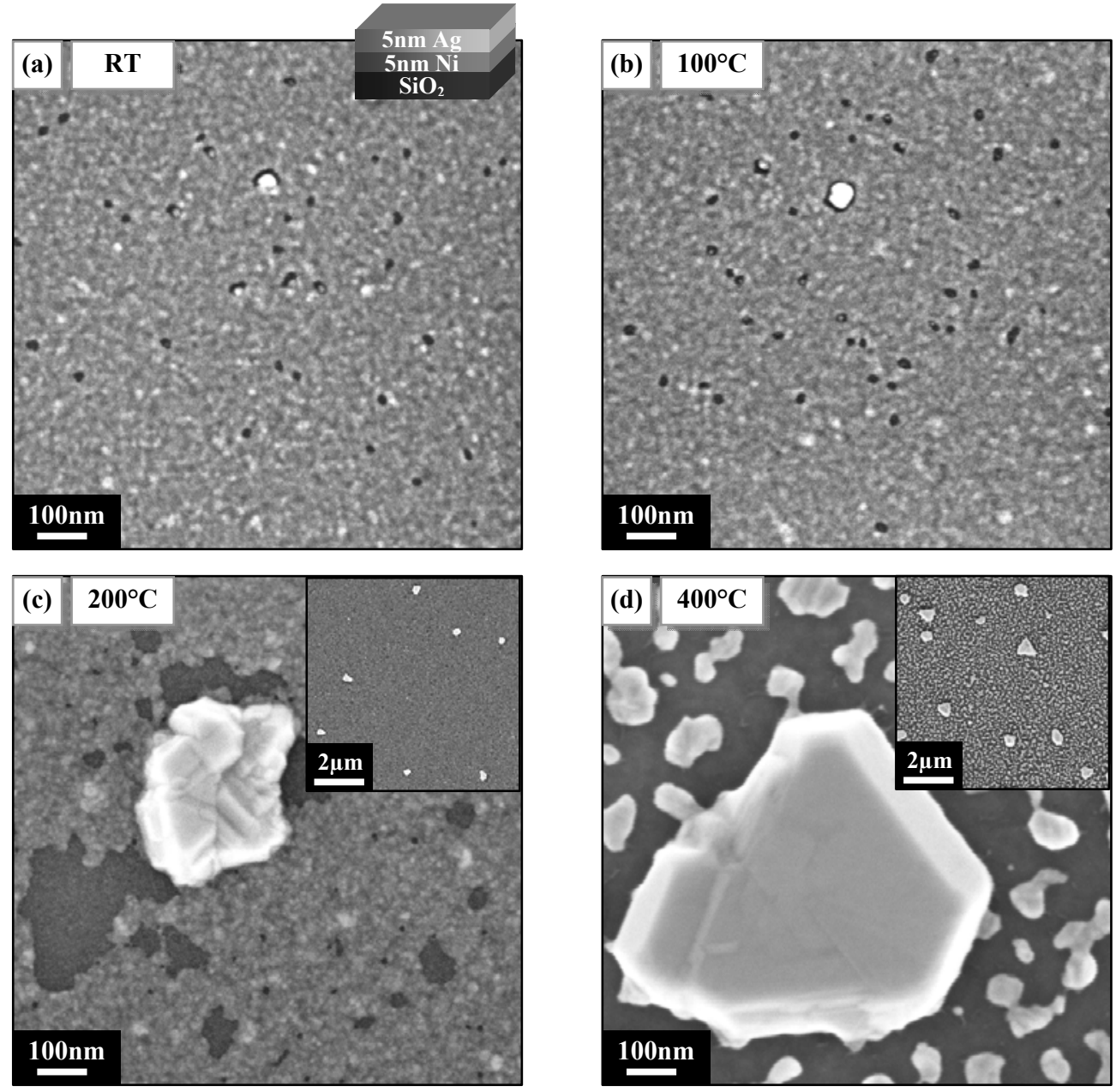

Abbildung 5.16: REM-Aufnahmen bei Raumtemperatur aufgedampfter und bei unterschiedlichen Temperaturen für $1 \mathrm{~h}$ ausgelagerter $5 \mathrm{~nm}-\mathrm{Ag} / 5 \mathrm{~nm}-\mathrm{Ni}$-Doppellagen.
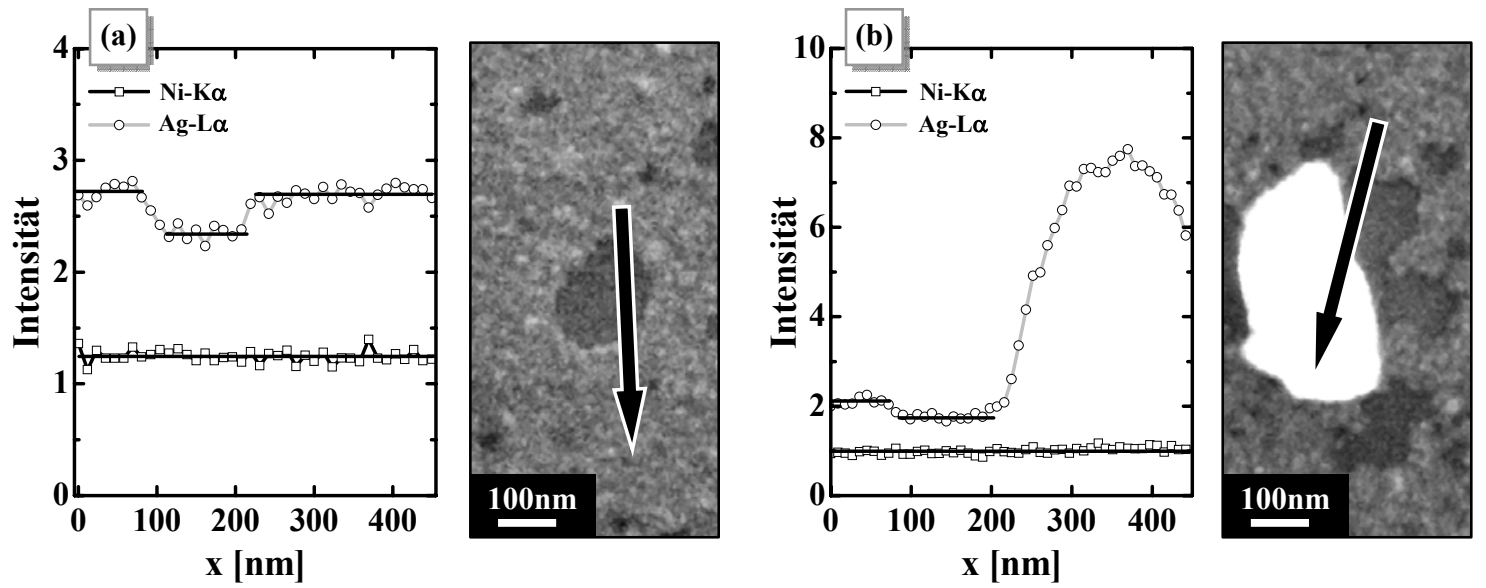

Abbildung 5.17: EDX-Linienprofile entlang der Oberfläche der bei $200^{\circ} \mathrm{C}$ ausgelagerten $\mathrm{Ag} / \mathrm{Ni}$-Doppellage. 
eine Entnetzung der Silberschicht handelt, während die darunter liegende Nickelschicht intakt bleibt. Zudem konnte mittels XPS kein Silizium nachgewiesen werden.

Durch Temperaturbehandlung bei $400^{\circ} \mathrm{C}$ entnetzt auch die Nickelschicht (Abbildung 5.16(d)) und bildet zwischen den Silberpartikeln eher verzweigte Inseln, was anzeigt, dass der Agglomerationsprozess noch nicht soweit fortgeschritten ist wie bei $600^{\circ} \mathrm{C}$ Auslagerungstemperatur der reinen Nickelschicht (Abbildung 5.15(a)). Zudem ist nicht auszuschließen, dass das Silber beim Entnetzen einen dünnen Film auf der Nickelschicht, einen so genannten Wetting-Layer, oder Silberatome in den Korngrenzen zurücklässt, was möglicherweise das Agglomerationsverhalten beeinflusst.

Die Untersuchungen haben gezeigt, dass die großen Silberpartikel auf einen Entnetzungsvorgang der zuvor geschlossenen Schicht zurückgehen. Bei der kodeponierten NiAgSchicht befindet sich jedoch nur ein Teil des Silbers an der Oberfläche und bildet vermutlich keine geschlossene Schicht.

Nickel auf Silber: Um zu klären, wie sich das System verhält, wenn sich die Silberschicht unterhalb einer Nickelschicht befindet, wurde eine $5 \mathrm{~nm}-\mathrm{Ni} / 5 \mathrm{~nm}-\mathrm{Ag}$-Doppellage bei Raumtemperatur deponiert und für eine Stunde bei $600^{\circ} \mathrm{C}$ ausgelagert. Wie bereits gesehen bildet das Silber keinen geschlossenen Film auf $\mathrm{SiO}_{2}$, sondern isolierte Inseln. Diese Inselstrukturen sind auch nach Deposition von Nickel noch zu erkennen (Abbildung 5.18(a)). Mittels XPS-Analyse wird eine Oberflächenkonzentration von ca. 12\% Silber gemessen, ohne dass Silizium nachgewiesen werden konnte. Das Nickel bedeckt somit die Flächen des zuvor freiliegenden Substrats sowie einen wesentlichen Teil des Silbers. Nach thermischer Behandlung zeigt sich auch hier ein Gefüge aus großen Partikeln mit bis zu $600 \mathrm{~nm}$ Durchmesser und weitaus kleineren Inseln (Abbildung 5.18(b)). EDX-Linienprofile belegen, dass die großen Partikel weitestgehend aus Silber bestehen und die kleinen Inseln ein deutliches Nickelsignal aufweisen (Abbildung 5.19). Weiterhin lassen die Messungen an
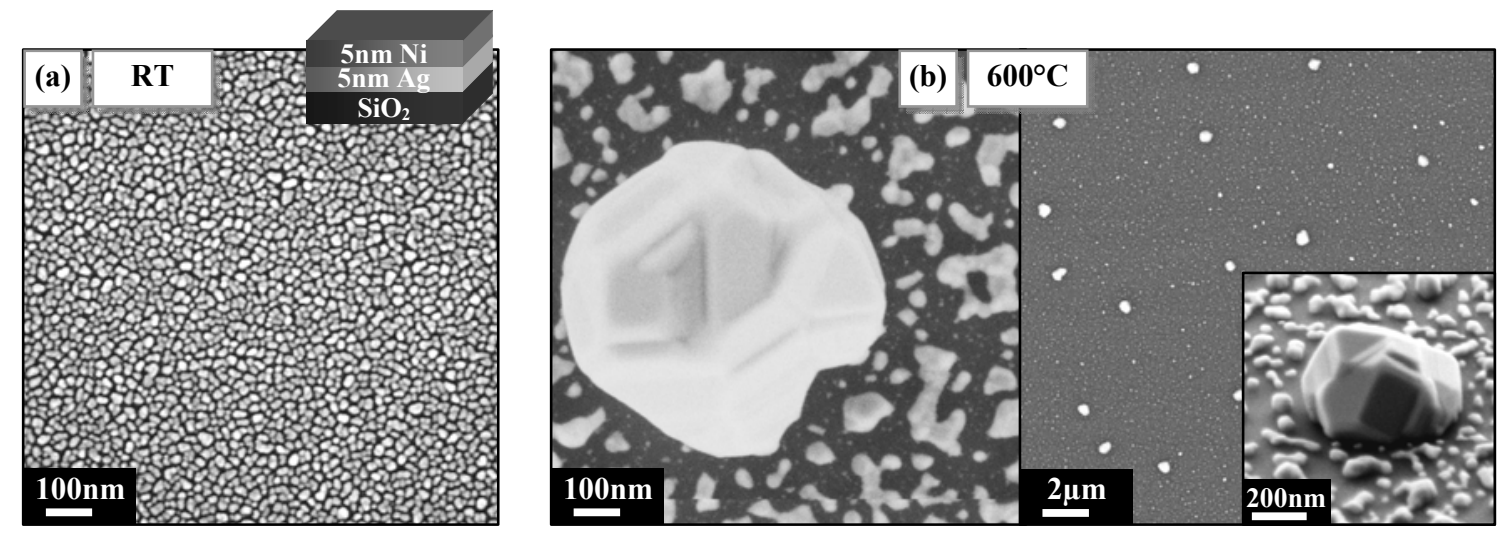

Abbildung 5.18: REM-Aufnahmen einer (a) bei Raumtemperatur aufgedampften und (b) bei $600^{\circ} \mathrm{C}$ für $1 \mathrm{~h}$ ausgelagerten $5 \mathrm{~nm}-\mathrm{Ni} / 5 \mathrm{~nm}-\mathrm{Ag}$-Doppellage. 

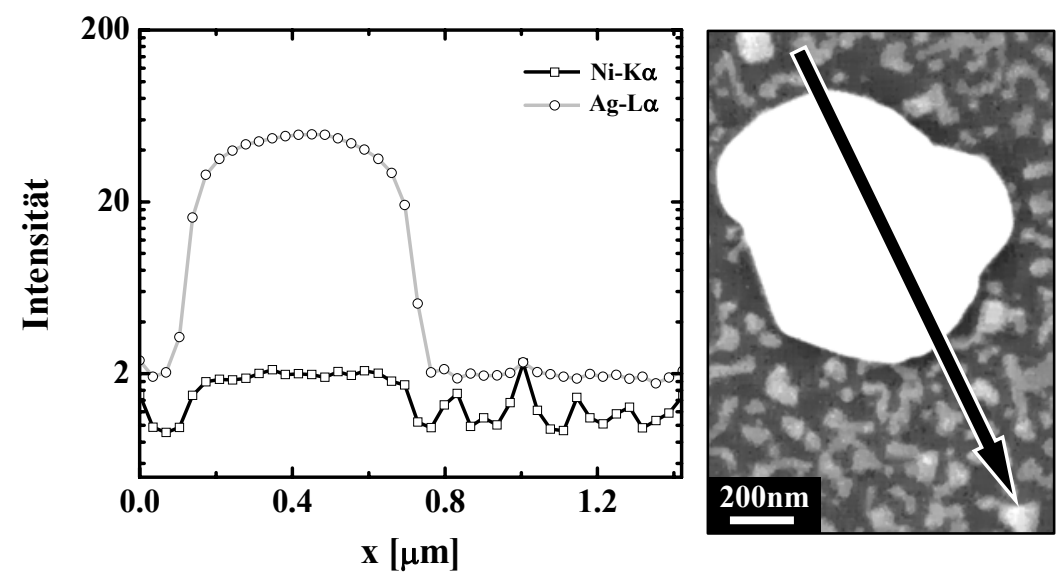

Abbildung 5.19: EDX-Linienprofil der wärmebehandelten Probe in logarithmischer Auftragung.

den Orten der Silberpartikel eine zusammenhängende Nickelschicht erkennen, die in Anbetracht der starken Tendenz zur Oberflächensegregation vermutlich direkt dem Substrat aufliegt.

\subsection{Diskussion}

\subsubsection{NiAg-Schichten auf Rippelstrukturen}

Prozesse der Materialorganisation: Die Untersuchungen an kodeponierten NiAgSchichten auf rippelstrukturierten Oberflächen haben gezeigt, dass infolge der starken Tendenz zur Oberflächensegregation des Silbers zwar keine laterale Ordnung der Phasen erzielt werden konnte, die Substratmorphologie jedoch sehr wohl einen deutlichen Einfluss auf die Materialorganisation während des Schichtwachstums besitzt. Ein vergleichbarer Einfluss konnte in Experimenten an dünnen Goldschichten auf ähnlich strukturierten Siliziumsubstraten nicht nachgewiesen werden [Umb06]. Diese Diskrepanz lässt sich vermutlich auf die größere Affinität des Nickels zum Sauerstoff der $\mathrm{SiO}_{2}$-Oberfläche und somit höhere Bindungsenergie der Adatome zurückführen, was im Folgenden noch genauer erläutert werden soll.

Die komplexen Prozesse beim Schichtwachstum können zu einer Vielzahl unterschiedlicher Oberflächenstrukturen führen. Auf den Templates kommt hierbei der Oberflächenkrümmung eine wesentliche Rolle zu. Obwohl eine Positionierung der Partikel in den Tälern auf Grund der Minimierung der Inseloberfläche bei gleichzeitiger Ausbildung der maximalen Anzahl von Bindungen zum Substrat den thermodynamisch stabilsten Zustand darstellt, kann der kinetische Pfad des Selbstorganisationsprozesses bei Temperaturen deut- 
lich unterhalb des Schmelzpunktes durchaus unterschiedliche metastabile Zustände enthalten und somit das Erreichen eines stabilen Zustands unterbinden. Eine entsprechende Untersuchung erfordert die Betrachtung der Prozesskinetik sowie die Analyse der auftretenden Mechanismen der Materialorganisation. Entscheidend für die Position der Kristallite ist das Frühstadium des Schichtwachstums, bei dem einzelne Adatome auf der Oberfläche diffundieren und sich präferentiell an Defekten oder anderen Adatomen anlagern. Der Diffusionskoeffizient ist gegeben durch

$$
\mathrm{D}=\frac{1}{2} \mathrm{a}^{2} v_{0} \exp \left(-\frac{\mathrm{E}_{\mathrm{d}}}{\mathrm{k}_{\mathrm{B}} \mathrm{T}}\right)
$$

wobei a den Abstand zweier benachbarter Potentialmulden, $v_{0}$ die Anklopffrequenz und $\mathrm{E}_{\mathrm{d}}$ die Aktivierungsenergie des Diffusionsprozesses bezeichnet. Die Lage des Adatoms wird hierbei durch das Ausbilden möglichst vieler Bindungen, was einer tiefen Potentialmulde entspricht, energetisch stabilisiert. Sind Bindungen zwischen Adatomen energetisch deutlich bevorzugt, bilden sich verstärkt Keime und es kommt zum dreidimensionalen Wachstum isolierter Inseln, wobei die Wechselwirkung zum Substrat und folglich der Einfluss der Oberflächentopographie eher gering ist.

Auf den strukturierten Templates kommt es im Gegensatz zur glatten Substratoberfläche zu einem gerichteten Materialtransport in Form von krümmungsinduzierter Oberflächendiffusion. Krümmungsgradienten stehen in linearem Zusammenhang mit Gradienten des chemischen Potentials [Her51], wodurch die Oberflächenmodulation einen Diffusionsstrom entlang des Krümmungsgradienten von den Bergkämmen in die Täler zur Folge hat (Abbildung 5.20). Konvexe (bzw. negative) Oberflächenkrümmung $\mathrm{K}=\left[\mathrm{d}^{2} \mathrm{~h} / \mathrm{dx}^{2}\right] /\left(1+[\mathrm{dh} / \mathrm{dx}]^{2}\right)^{3 / 2}<0$ führt $\mathrm{zu}$ einer geringeren Koordination. In den Tälern können ent-

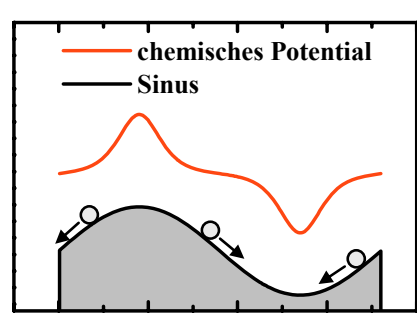

laterale Koordinate

Abbildung 5.20: Chemisches Potential auf einer sinusförmigen Oberfläche. sprechend mehr Bindungen eingegangen werden und der damit verbundene Energiegewinn ist um so größer, je höher die Bindungsenergie ist. Auf Grund der größeren Affinität zum Sauerstoff des $\mathrm{SiO}_{2}$ besitzt Nickel eine stärkere Bindung zum Substrat, sodass der Einfluss der Oberflächenmorphologie beim vergleichsweise inerten Gold folglich geringer ausfällt. Demgegenüber wird die Kinetik des Selbstorganisationsprozesses durch stärkere Bindung der Adatome zum Substrat und der somit höheren Aktivierungsenergie $\mathrm{E}_{\mathrm{d}}$ eingeschränkt, sodass die Sprungwahrscheinlichkeit zwischen den einzelnen Diffusionsschritten und damit die mittlere Diffusionslänge abnimmt. Voraussetzung für eine präferentielle Anlagerung der Adatome in den Tälern ist demzufolge eine nicht zu große Grenzflächenenergie zwischen Deposit und Substrat sowie eine hohe Substrattemperatur. 
Haben sich stabile Keime gebildet, so sind diese eher immobil, da trotz Migration mehratomiger Cluster die Mobilität mit der Clustergröße stark abnimmt [Bas76]. Die in der Literatur ausgiebig behandelten Prozesse des Inselwachstums und der Koaleszenz [Smi95, Tho00] führen jedoch auch im weiteren Stadium des Schichtwachstums zu morphologischen Veränderungen. Während das System versucht, den Aufwand an Oberflächen- und Grenzflächenenergie zu minimieren, herrscht eine starke treibende Kraft zur Vergröberung durch Oberflächendiffusion und Korngrenzenmigration. Es kommt zur Koaleszenz direkt benachbarter Inseln, wodurch vergrößerte, oft einkristalline Inseln entstehen. Ein weiterhin häufig angeführter Prozess ist die sogenannte Ostwaldreifung, bei der größere Inseln infolge des günstigeren Oberflächen-zu-Volumen-Verhältnisses auf Kosten kleinerer wachsen, ohne dass diese in direktem Kontakt stehen.

Die signifikante Reduktion der Inselgröße auf den rippelstrukturierten Substraten indiziert, dass nicht allein das Frühstadium des Schichtwachstums für die resultierende Oberflächenmorphologie verantwortlich ist. Die Bergrücken wirken auf Grund der negativen Krümmung wie Diffusionsbarrieren und verhindern ein Zusammenwachsen der Inseln über die Erhebungen hinaus. Die schwache hexagonale Nahordnung der 20nm NiAg-Schicht (Abbildung 5.4(b)) bei nahezu einheitlichem Inseldurchmesser zeigt dennoch eine Wechselwirkung isolierter benachbarter Inseln auf und deutet auf eine Überlappung der Einflussbereiche großer Inseln und die Auflösung kleinerer Inseln durch Koaleszenz bzw. Ostwaldreifung. Anhand der überwiegend kompakten, runden Inseln der 20nm NiAg-Schicht wird deutlich, dass die uniaxiale geometrische Anisotropie der Substratoberfläche nicht auf die Form der Inseln übertragen wird, sondern die Minimierung der freien Oberflächenenergie die resultierende Geometrie dominiert. Diese Dominanz der Agglomeration ist ebenfalls thermisch aktiviert und ein Effekt der erhöhten Substrattemperatur. Sie nimmt jedoch mit zunehmender Inselgröße und folglich sinkendem Oberflächen-zu-Volumen-Verhältnis ab (siehe Anhang C). Die Deposition zusätzlichen Materials (Abbildung 5.1(a)) bewirkt hingegen keine Ausbildung länglicher Inseln entlang der Täler mit erhöhtem Aspektverhältnis, sondern ein Überwachsen der Bergkämme und den Verlust lateraler Ordnung.

Im Frühstadium des Wachstums folgen die Inseldurchmesser dünner Schichten häufig nahezu einer logarithmischen Normalverteilung [Bla94]. Die sich hier ergebende bimodale Verteilung resultiert vermutlich aus sekundärer Nukleation, wie sie beispielsweise von Pashley et al. [Pas64] bei Deposition von Silber auf $\mathrm{MoS}_{2}$ beobachtet wurde. Die Koaleszenz führt zur Bildung größerer und höherer Inseln, die weniger Platz auf der Oberfläche in Anspruch nehmen. In den Zwischenbereichen entstehen Löcher und Kanäle, die neue Nukleationspunkte bieten und durch sekundäre Nukleation zusätzlicher Keime bedeckt werden. Ein zweiphasiges Verhalten ist als Ursache der Aufspaltung der Inseldurchmesserverteilung in Anbetracht der starken Oberflächensegregation des Silbers kaum denkbar, auch wenn die scheinbar regellose Anordnung der kleineren Partikel in Anlehnung an die oben ausgeführten Überlegungen bezüglich des unterschiedlichen Diffusionsverhaltens von Nickel- und Goldadatomen auf nur schwach an das Substrat gebundene Silberinseln hin- 
deuten könnten. Da jedoch die Zusammensetzung der kleinen Inseln nicht bestimmt werden konnte, sind entsprechende Überlegungen spekulativ und hier nicht zu belegen.

Magnetisierung: Nanopartikel mit einem geringeren Durchmesser als etwa 30nm befinden sich häufig in einem Eindomänenzustand [Far05]. Dieser Größeneffekt kann bei Durchmessern isolierter magnetischer Teilchen unterhalb von etwa 10nm [Lop02] und nur geringer Anisotropie zur Ausbildung eines superparamagnetischen Verhaltens führen, wobei die thermische Energie die Anisotropieenergie übersteigt und die Magnetisierungsrichtung isolierter Teilchen fluktuiert.

Da der Übergang zum Superparamagnetismus eine verschwindende Remanenz zur Folge hat und die Hysteresekurven der 20nm NiAg-Schicht auf geordneter Rippelstruktur (Abbildung 5.9) ein ferromagnetisches Magnetisierungsverhalten anzeigen, ist eine maximale Größe magnetischer Nickelpartikel oberhalb des superparamagnetischen Limits anzunehmen. Allerdings können auch kleinere Partikel infolge der magnetostatischen Dipolkopplung ferromagnetisch ordnen [Sug97] und auf Grund der Schwierigkeit, Größeneffekte von Wechselwirkungseffekten zu differenzieren, kann bezüglicher der Größe der magnetischen Teilchen keine definitive Aussage getroffen werden.

Die fehlende Anisotropie bestätigt das auf Grund der geometrischen Anordnung weitestgehend symmetrischer Partikel mit in beiden untersuchten Richtungen vergleichbaren Abständen zu erwartende Verhalten. Nickeleinkristalle weisen im Allgemeinen eine magnetokristalline Anisotropie mit leichter Achse entlang der $<111>$-Richtungen auf. Anhand epitaktisch gewachsener dünner Filme wurden außerdem verschiedene uniaxiale Anisotropien in Schichtebene beobachtet, die meist auf mechanische Spannungen zurückzuführen sind [And61, Ued76]. Die hier nicht nachweisbare Kristallanisotropie deutet auf eine in Schichtebene zufällig ausgerichtete Kristallorientierung. Die parallele Ausrichtung einer kristallographischen Achse mit vierzähliger Symmetrie der kubischen Struktur wie beispielsweise der $<110>$-Richtung entlang der Rippelstruktur kann jedoch nicht vollständig ausgeschlossen werden. Röntgentexturmessungen an einem PHILIPS X'Pert Vierkreisdiffraktometer lieferten auf Grund des hohen Signalrauschverhältnisses infolge der geringen Schichtdicke und Kristallgröße diesbezüglich keine zusätzlichen Erkenntnisse.

Gesteuerte Selbstorganisation: Die Ergebnisse haben gezeigt, dass sich der Einfluss der Substratmorphologie auf die Selbstorganisation während des Schichtwachstums nicht allein im Frühstadium des Wachstums und damit bei der Keimbildung durch krümmungsinduzierte Oberflächendiffusion einzelner Adatome zeigt. Die Substratstrukturierung lässt auch maßgebliche Auswirkungen in späteren Stadien der Koaleszenz und Ostwaldreifung bereits bestehender Inseln erkennen. Die Periodizität der Substratoberfläche lässt hierbei unter der Voraussetzung einer an die Rippelwellenlänge und -tiefe angepassten Schichtdicke bzw. deponierten Materialmenge die Selektierung einer wohl definierten Inselgröße zu, 
deren Dimension und Verteilungsbreite sich mittels Parameteroptimierung weiter reduzieren lässt. Eine entsprechende Miniaturisierung der Strukturen erfordert jedoch aufwendigere Analyseverfahren und stellt einen technischen Aspekt dar, der nicht Teil dieser Arbeit ist. Der hier untersuchte Selbstorganisationsprozess erlaubt die gezielte Herstellung linearer Ketten isolierter metallischer bzw. magnetischer Nanopartikel mittels eines technisch prinzipiell einfachen Verfahrens.

\subsubsection{Thermische Behandlung von Ni-Ag-Schichten}

Die thermische Behandlung unterschiedlicher Ni-Ag-Schichten hat gezeigt, dass die Materialorganisation auf Grund gekoppelter Prozesse eine vom Verhalten reiner Silber- bzw. Nickelschichten stark abweichende Strukturbildung zur Folge hat. Das Silber besitzt unabhängig von seiner ursprünglichen Position - sei es nun auf, in oder unter der Nickelschicht - in Verbindung mit Nickel eine deutlich höhere Mobilität und agglomeriert zu Inseln groBen Durchmessers, wohingegen das Nickel auch ohne vorhandenes Silber eine Anordnung von kleineren, nahezu homogen verteilten Inseln ausbildet. Drei Prozesse konnten während der thermischen Behandlung der Ni-Ag-Schichten identifiziert werden, deren sukzessiver Ablauf die mikrostrukturelle Entwicklung der Filme in entscheidendem Maße bestimmt. Silber zeigt in Verbindung mit Nickel eine deutliche Oberflächensegregation und setzt sich somit auf die Nickelschicht, während eine $\mathrm{Ag}-\mathrm{SiO}_{2}-\mathrm{Grenzfläche} \mathrm{vermieden} \mathrm{wird.} \mathrm{Bildet} \mathrm{das}$ Silber zunächst einen bedeckenden Film auf einer zusammenhängenden Nickelschicht, so kommt es vermutlich überwiegend mittels Selbstdiffusion erst zur Entnetzung des Silberfilms (Abbildung 5.16). Hierbei lagern sich die aus den entstehenden Löchern gelösten AgAtome teilweise an weit entfernte Kristalle an, die zu einer beachtlichen Partikelgröße heranwachsen, bevor bei höheren Temperaturen auch das Nickel entnetzt. Für gewöhnlich wird das Material bei Nukleation und Wachstum von Löchern während der Entnetzung auch in fester Phase in direkter Umgebung wieder angelagert und zu einer Randwulst aufgeschüttet [Jir90, Won00], ähnlich wie es bei Flüssigkeiten durch die zurückweichende Entnetzungsfront infolge von viskosem Fließen beobachtet wird [See01]. Für eine ausführlichere Behandlung des Mechanismus der Entnetzung sowie die Identifizierung des vorherrschenden Transportprozesses soll jedoch an dieser Stelle auf das anschließende Kapitel verwiesen werden.

Wesentlichen Einfluss auf die Strukturbildung während thermischer Behandlung hat der Ausgangszustand des Materials. Liegt zunächst ein zusammenhängender Film vor, so besteht die Möglichkeit der Entnetzung. Sind bereits während bzw. nach der Deposition ausschließlich isolierte Inseln vorhanden wie im Falle der reinen Silberschicht auf $\mathrm{SiO}_{2}$ (Abbildung 5.15(b)), so sind diese weitestgehend immobil. Daher ist das resultierende Gefüge der erst nachträglich wärmebehandelten Ni-Ag-Schichten von dem bei hoher Temperatur kodeponierter NiAg-Schichten klar zu differenzieren. Jedoch weist auch die bei 

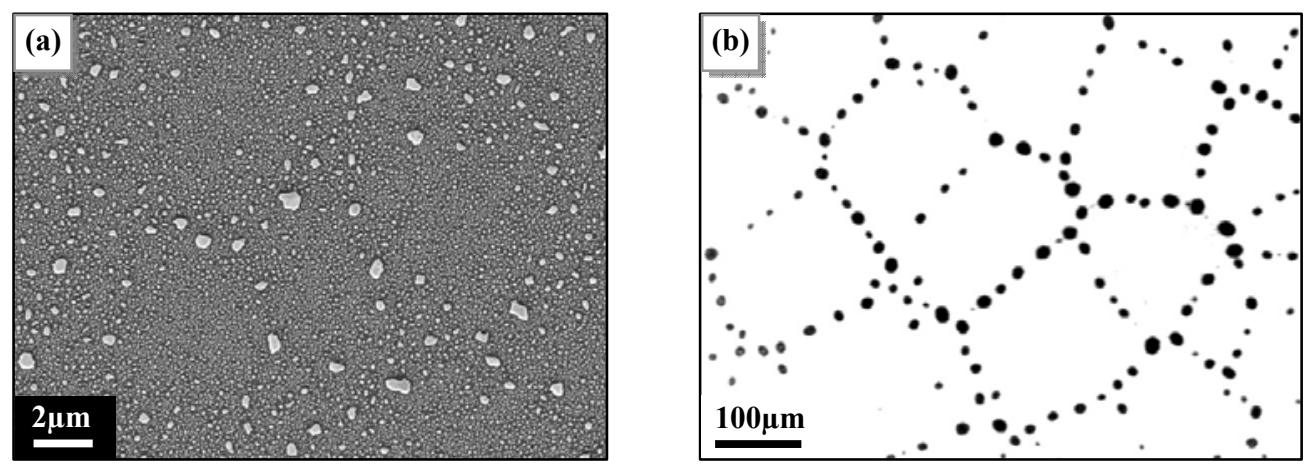

Abbildung 5.21: (a) Bei Raumtemperatur kodeponierte und anschließend für $1 \mathrm{~h}$ bei $600^{\circ} \mathrm{C}$ thermisch behandelte $10 \mathrm{~nm} \mathrm{NiAg-Schicht} \mathrm{sowie} \mathrm{(b)} \mathrm{entnetzter} 45 \mathrm{~nm}$ Polystyrolfilm (schwarz) auf Silizium [Rei92].

Raumtemperatur kodeponierte NiAg-Schicht vermutlich keine durchgehende Silberoberfläche auf, wie das deutliche Nickelsignal der XPS-Analyse bekräftigt, sodass auch hier nicht ohne Weiteres eine Entnetzung mittels reiner Selbstdiffusion stattfinden kann. Die netzartige Anordnung größerer Inseln nach Temperaturbehandlung ähnelt indes stark der Struktur, wie sie beispielsweise nach der Entnetzung von Polymerfilmen beobachtet wird [Rei92] (Abbildung 5.21).

Noch stärker drängt sich die Frage nach dem Prozessablauf bei der thermisch behandelten $5 \mathrm{~nm}-\mathrm{Ni} / 5 \mathrm{~nm}-\mathrm{Ag} / \mathrm{SiO}_{2}$-Probe auf (Abbildung 5.18), bei der die Silberschicht anfangs unter der Nickelschicht begraben ist. Ein Herauswachsen der Silberpartikel durch Ostwaldreifung in der Nickelschicht erfordert Volumendiffusion über weite Strecken, was bei der starken Tendenz zur Oberflächensegregation und der geringen Schichtdicke auszuschließen ist. Auf Grund der im Allgemeinen höheren Aktivierungsenergie von Volumen- und Grenzflächendiffusion gegenüber Oberflächenprozessen ist die Initiierung der Agglomeration zu größeren Silberpartikeln folglich erst nach der Oberflächensegregation anzunehmen. Im Folgenden sollen mögliche Szenarien hinsichtlich der mikrostrukturellen Entwicklung kurz diskutiert werden:

- Silber reichert sich in den Korngrenzen des Nickels an, unterbricht die Nickelschicht und diffundiert an die Oberfläche, bzw. das Nickel diffundiert in die Grenzfläche zwischen Silber und Substrat. Ein ähnliches Verhalten wurde von Sandström et al. an Ag/Ni-Multilagen beobachtet [San99]. Dabei bewirkt die Wärmebehandlung zunächst ein Glätten der Grenzflächen, während im weiteren Verlauf der Prozedur das Silber in die Korngrenzen der Nickelfilme diffundiert und die horizontale Lagenstruktur schließlich vollständig zerstört. Ein solcher Vorgang führt jedoch dazu, dass bereits bestehende isolierte Silberpartikel an unterschiedlichen Orten aus der Nickelschicht wachsen. Es entsteht folglich kein zusammenhängender Silberfilm auf der Oberfläche und die Bildung der groBen Silberpartikel durch reine Entnetzung ist ausgeschlossen. 
- Einzelne Silberatome gelangen durch Korngrenzendiffusion an die Oberfläche und diffundieren auf der Nickeloberfläche, wobei es zu Keimbildung und Wachstum kommt. Da sich an den Korngrenzen polykristalliner Filme mit steigender Temperatur verstärkt tiefe Furchen bilden [Mul57] und diese für die Silberatome die größtmögliche Koordination versprechen, dürfte das Silber hier allerdings quasi festhängen. Eine Diffusion entlang der Furchen ist hingegen nicht ausgeschlossen.

Die Untersuchungen zeigen, dass Silber das Nickel im thermodynamischen Gleichgewicht im hier untersuchten Temperaturbereich nicht benetzt und in einer entsprechenden Matrix an die Oberfläche getrieben wird, wohingegen Nagesh und Pask [Nag83] in Experimenten mit flüssigen Silbertropfen auf kristallinem Nickel einen Benetzungswinkel von nur $9^{\circ}$ beobachten konnten. Weiterhin besteht eine treibende Kraft zur Entnetzung des Nickels auf der $\mathrm{SiO}_{2}$-Substratoberfläche. Die Frage des Vorgangs der Oberflächensegregation in dem durch Grenzflächen dominierten Dünnschichtsystem bleibt hier jedoch offen. Anhand von Bulk-Proben ist indes bekannt, dass ein geringer Nickelanteil in Silber zu einer Art Sandwichstruktur mit einer dünnen Nickelschicht oder Nickelpartikeln dicht unterhalb der Oberfläche führt [Rol85, Tre90].

Die Bildung der großen Partikel in beträchtlichem Abstand bei nur wenigen Löchern in der Silberschicht der bei $200^{\circ} \mathrm{C}$ ausgelagerten Ag/Ni-Doppellage (Abbildung 5.16(c)) deutet darauf, dass auch hier kein reiner oder gewöhnlicher Entnetzungsprozess vorliegt. Die Formung derartiger Strukturen wurde bei entsprechender Entnetzung dünner metallischer Filme in fester Phase, soweit hier bekannt ist, bislang nicht berichtet. Offensichtlich ist jedoch ein zugrunde liegender zunächst geschlossener Metallfilm, auf dem die Silberatome diffundieren, eine notwendige Voraussetzung. Die in Abschnitt 5.1 behandelten, bei erhöhter Substrattemperatur kodeponierten NiAg-Schichten, welche zu keiner Zeit einen geschlossenen Film gebildet haben, weisen derartige Strukturen nicht auf. 


\section{Kapitel}

\section{Selbstorganisation durch Entnetzung}

Im Gegensatz zu dem vorangegangenen Kapitel, in dem der Selbstorganisationsprozess durch die Materialdeposition initiiert wurde, während die Rahmenbedingung einer erhöhten thermischen Energie der Adatome auf der Substratoberfläche von Beginn an gegeben war, wird in diesem Kapitel ein anderer Mechanismus der Prozessaktivierung thematisiert. In den folgenden Experimenten ist das Material in Form eines geschlossenen Films auf der Substratoberfläche bereits vorhanden und die Aktivierung des strukturbildenden Selbstorganisationsprozesses geschieht über die Erhöhung der thermischen Energie, wodurch es zur Entnetzung in fester Phase kommt. Obwohl in beiden Fällen - wird einmal von dem Vorhandensein zweier Phasen abgesehen - das aus der Gasphase abgeschiedene Material prinzipiell einen vergleichbaren Ausgangszustand besitzt, in Form von stochastisch verteilter Materialdeposition und Temperatureinwirkung dieselben Prozessierungsschritte vorliegen und auch der thermodynamische Gleichgewichtszustand quasi identisch ist ${ }^{14}$, bewirkt die erst nach Deposition erhöhte Temperatur, dass das System einen anderen kinetischen Pfad durchläuft und somit unterschiedliche metastabile Zustände zugänglich sind.

Für die gezielte Herstellung von Nanostrukturen über den Prozess der Entnetzung ist ein grundlegendes Verständnis des Entnetzungsmechanismus von entscheidender Bedeutung. Im Folgenden soll zunächst ein kurzer Überblick über bisher bekannte physikalische Ursachen und Mechanismen gegeben werden, bevor die Ergebnisse der hier geführten Experimente bezüglich morphologischer Entwicklung und Kinetik während der Entnetzung am Beispiel dünner Nickelschichten dargestellt werden. Anschließend wird anhand entsprechender Filme auf rippelstrukturierten Substraten der Frage nachgegangen, ob mittels eines gesteuerten Selbstorganisationsprozesses über das spezielle Oberflächenprofil die Formation von Nickelnanostäben oder -nanodrähten induziert werden kann. Schließlich wird der Einfluss einer nicht mischenden Komponente durch die Zugabe von Silber untersucht.

\footnotetext{
${ }^{14}$ Wird einmal von der Desorption während der Deposition und Auslagerung bei höherer Temperatur im Vakuum abgesehen, so besteht der thermodynamische Gleichgewichtszustand in beiden Fällen aus isolierten kompakten Inseln des gesamten deponierten Schichtmaterials auf der Substratoberfläche, wobei im eigentlichen Sinne eine einzige große Insel den Zustand geringster freier Energie darstellt.
} 


\subsection{Grundlagen zur Entnetzung}

Dünne Filme besitzen ein hohes Grenzflächen-zu-Volumen-Verhältnis, weshalb ihre Geometrie maßgeblich durch die Eigenschaften der auftretenden Grenzflächen bestimmt wird. $\mathrm{Ob}$ ein glatter, zusammenhängender Film energetisch stabil ist, kann unter Vernachlässigung mechanischer Spannungen und der Voraussetzung eines nicht verformbaren Substrats anhand der von Young [You05] formulierten Bedingung zur Minimierung der freien Energie abgeleitet werden:

$$
\gamma_{\mathrm{SV}}=\gamma_{\mathrm{SL}}+\gamma_{\mathrm{LV}} \cos \theta
$$

Hierbei bezeichnet $\gamma$ die jeweilige Grenzflächenenergie nach Abbildung 6.1 und $\theta$ den Gleichgewichtsbenetzungswinkel. Ist der Benetzungswinkel größer null und somit die Oberflächenenergie des Substrats kleiner als die Summe aus der Grenzflächenenergie zwischen Film und Substrat und der Oberflächenenergie des Films, besteht eine treibende Kraft zur Entnetzung. Genau genommen muss hierbei der durch die Ausbildung einzelner Tropfen mit Radius $r$ veränderte Beitrag an Grenz-

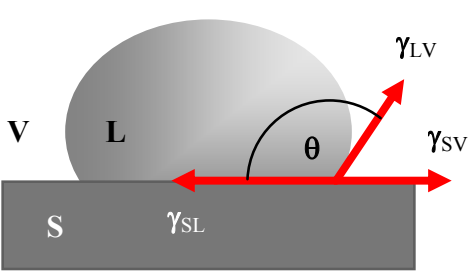

Abbildung 6.1: Dreiphasensystem fest (S), flüssig (L), gasförmig (V). flächen berücksichtigt werden, sodass die Stabilitätsbedingung für einen benetzenden Film der Dicke d unter der Annahme einer einheitlichen resultierenden Tropfengröße folgende Form annimmt [Sro95]:

$$
\frac{r}{d}<\frac{3 \sin \theta}{1-\cos \theta}
$$

Der Tropfenradius wird hierbei indes kinetisch determiniert und obwohl eine energetische Stabilitätsanalyse bei der quantitativen Vorhersage dynamischer Phänomene meist versagt, so liefert sie im Allgemeinen doch eine qualitativ zutreffende Beschreibung, die es erlaubt, die entscheidenden Variablen zu identifizieren. Das Konzept der Minimierung freier Energie gilt streng genommen nur im thermodynamischen Gleichgewicht und der Übergang in den Gleichgewichtszustand wird gewöhnlich von kinetisch limitierenden Faktoren beeinflusst. Folglich drängt sich die Frage auf, welche Mechanismen letztlich für das Aufbrechen eines geschlossenen Films verantwortlich sind.

Die Physik der Entnetzung ist gegenwärtig ein aktives Forschungsgebiet [Mug05, Sut06, Pie07], wobei die Grundzüge des Effektes bei dünnen Flüssigkeitsfilmen weitestgehend verstanden sind. Es wurden zwei unterschiedliche Mechanismen identifiziert, die zur Initiierung des Entnetzungsprozesses führen können. Einerseits besteht die Möglichkeit der Nukleation von Löchern an Defekten [Red91, Bis96]. Jede Oberfläche weist Inhomogenitä- 
ten, Verunreinigungen und Defekte auf. Diese können Nukleationskeime der zur Entnetzung erforderlichen Löcher in dem bedeckenden Film bilden. Weiterhin können auch strukturelle sowie chemische Inhomogenitäten des Films Nukleationspunkte zur Lochbildung darstellen [See01a]. Die Löcher reichen bis auf die Substratoberfläche und legen im Zuge ihres Wachstums immer größere Flächen des Substrats frei.

Auf der anderen Seite kann es zur sogenannten spinodalen Entnetzung [Bro90] kommen. Spinodale Entnetzung beruht auf thermischen Oberflächenfluktuationen bzw. Filmdickenschwankungen, bei der die Amplitude in einem schmalen Wellenlängenbereich abhängig von den Grenzflächenenergien und interatomaren Wechselwirkungen anwächst und der Film an periodischen Punkten unterbrochen wird, sodass zumindest im Anfangsstadium des Entnetzungsvorgangs ein Bild aus korrelierten Strukturen entsteht. Dies Phänomen wird häufig bei Polymerfilmen beobachtet, bei denen sich auf Grund der Dominanz langreichweitiger van der Waals-Kräfte die Grenzfläche im Wechselwirkungsbereich der Oberfläche befindet und zu einem entsprechenden Aufrauungsterm führt, während der durch die Oberflächenspannung vermittelte Laplace-Druck demgegenüber die glättende Rückstellkraft darstellt, und ist stark von der Filmdicke abhängig. Herminghaus et al. konnten einen entsprechenden Effekt indes auch an flüssigen Goldfilmen nachweisen [Her98].

Die heterogene Nukleation von Löchern ist de facto kein reiner Dreckeffekt sondern eher der Normalfall und lässt sich auch bei Verwendung hoch reiner Materialien nicht vermeiden. Abbildung 6.2 zeigt eine lichtmikroskopische Aufnahme eines entnetzenden 40nm dicken Flüssigkristallfilms auf Silizium [Her98]. Neben spinodaler Störungen in Form korrelierter Strukturen mit einer Wellenlänge von etwa $10 \mu \mathrm{m}$ sind auch kreisförmige Löcher zu beobachten, die von Nukleation an Defekten herrühren. Die Störung befindet sich hierbei an der Filmoberfläche und die hellen Zwischenbereiche der Wellenstrukturen besitzen noch eine Schichtdicke von etwa 30nm. Ob die spinodale Entnetzung wirklich eine signifikante Rolle beim Aufbrechen dünner Polymerfilme spielt, ist jedoch umstritten [Jac98] und die genaue Natur der Nukleationskeime meist ungeklärt.

Ist ein Loch erst einmal entstanden, so bildet das aus

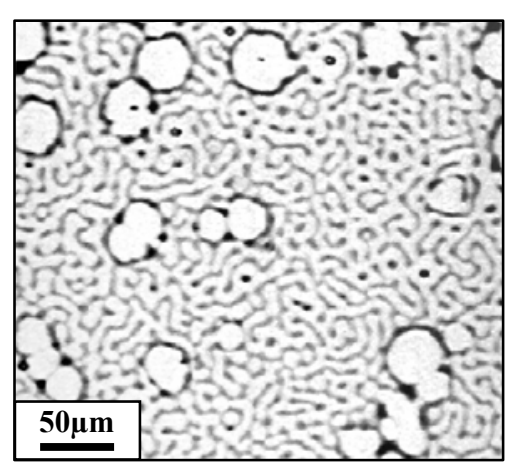

Abbildung 6.2: Lichtmikroskopische Aufnahme eines Flüssigkristallfilms auf Silizium [Her98]. dem Lochbereich entfernte Material einen leicht erhöhten, nahezu toroidalen Rand, der das Loch begrenzt. Auf der filmzugewandten Seite geht diese Randwulst in die unberührte Schicht über und es kommt zu einer konkaven Oberflächenkrümmung (Abbildung 6.3), die auf Grund des resultierenden negativen Laplace-Drucks einen zur Senke gerichteten Materialtransport zur Folge hat. Genauere Untersuchungen [See01] haben gezeigt, dass diese Störung der Flüssigkeitsoberfläche infolge der viskosen Eigenschaften wie eine gedämpfte harmonische Schwingung abfällt, während das Zusammentreffen der Senke mit der Sub- 
stratoberfläche bei voranschreitender Entnetzungsfront zum Abreißen der Randwulst und anschließender Neubildung führen kann [Won00].

Für die Betrachtung der Wachstumsrate entnetzter Flächen muss zwischen zwei Fällen differenziert werden. Redon et al. [Red91] beobachteten anhand von $50 \mu \mathrm{m}$ dicken Ölfilmen auf Silizium, dass sich die Wachstumsrate $\mathrm{v}=\mathrm{dR} / \mathrm{dt}$ unabhängig vom Lochradius $R$ und antiproportional zur Viskosität $\eta$ verhält. Später konnten Brochard-Wyart et al. [Bro94] zeigen, dass

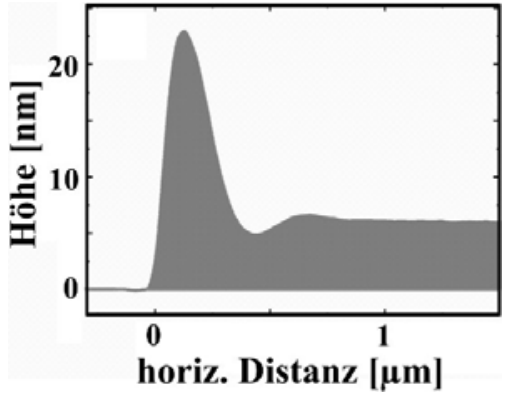

Abbildung 6.3: AFM-Aufnahme der Randwulst eines Polystyrolfilms auf Silizium. Nach [See01]. dies nur für nicht gleitende Filme ${ }^{15}$ gilt, während bei stark gleitenden Filmen der Radius mit $\mathrm{R}(\mathrm{t}) \propto \mathrm{t}^{2 / 3}$ anwächst.

Im Spätstadium der Entnetzung treffen die Löcher aufeinander und die Randwülste benachbarter Löcher werden zu langen zylinderartigen Bändern zusammen geschoben [See05]. Wie von der Rayleigh-Instabilität des Wasserstrahls bekannt ist [Ray78], sind zylinderförmige Flüssigkeitsoberflächen dynamisch instabil und zerfallen in einzelne Tropfen. Hierdurch entsteht ein polygonales Netz aus isolierten Flüssigkeitstropfen auf der Substratoberfläche (Abbildung 5.21(b)).

Auch Filme fester Phase können eine thermische Instabilität zeigen und sich durch einen Agglomerationsprozess in isolierte Inseln transformieren. Auf Grund der technologischen Relevanz polykristalliner metallischer Filme auf dielektrischen Substraten wird ihre thermische Stabilität seit langem erforscht [Pre72]. Obwohl es viele Parallelen zu Entnetzung von Flüssigkeiten gibt, ist das Phänomen der Entnetzung hier deutlich komplexer und bislang nicht im Detail geklärt. Polykristalline Filme sind nicht atomar glatt, auf atomarer Skala anisotrop und besitzen infolge der feinen Mikrostruktur und des fein verzweigten Netzes aus Korngrenzen einen erhöhten Anteil an Grenzflächen. Die Art der Korngrenzen können vielfältig sein, da die einzelnen Kristalle meist unterschiedlich orientiert sind und somit unterschiedliche Kristallflächen aufeinander treffen. Diese Korngrenzen stellen die strukturelle Schwachstelle eines entsprechenden dünnen Films dar. Ähnlich wie an der zuvor betrachteten Dreiphasengrenze (Abbildung 6.1) ist das System nach kapillaritätstheoretischen Überlegungen bestrebt, an den Schnittlinien, an denen die Korngrenzen auf die freie Oberfläche treffen, ein Gleichgewicht der auftretenden Grenzflächenspannungen herzustellen, wodurch es zur Ausbildung von Furchen kommt (Abbildung 6.4). Analog zu Gleichung 6.1 ergibt sich die Beziehung

\footnotetext{
${ }^{15}$ In der Theorie der viskosen Hydrodynamik werden im Wesentlichen zwei Randbedingungen für die Bewegung der Dreiphasengrenze verwendet [Dus79]. Bei einem gleitenden Film geht die Geschwindigkeit des Fließprozesses in der Nähe der Grenzfläche nicht auf Null zurück, sondern weist an der Phasengrenze einen Sprung auf. Die "no slip"-Randbedingung verbietet hingegen ein Gleiten des Films. Experimentell werden beide Fälle beobachtet [Bro94].
} 


$$
\gamma_{\mathrm{Gb}}=2 \gamma_{\mathrm{FV}} \sin \theta
$$

wobei $\gamma_{\mathrm{Gb}}$ die Grenzflächenenergie der Korngrenze und $\gamma_{\mathrm{FV}}$ die Oberflächenenergie des Films bezeichnet. Mit steigender Temperatur werden Diffusionsprozesse angeregt und der Materialtransport aus den Furchen führt zu einer Erhöhung im Randbereich. Diese Erhöhungen stellen eine Störung der Oberfläche dar und werden mittels Oberflächendiffusion in Richtung des Krüm-

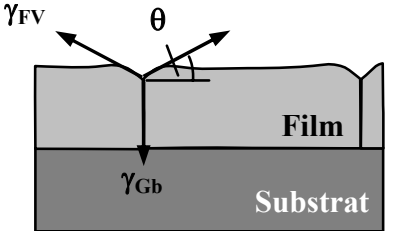

Abbildung 6.4: Furchenbildung an einer Korngrenze. mungsgradienten ausgeglättet, wodurch $\theta$ verringert und folglich die Furchen tiefer in die Korngrenzen getrieben werden [Mul57].

Trifft die Furche auf das Substrat, so entsteht ein Loch und es bildet sich ein Gleichgewichtswinkel an der Kontaktlinie zwischen Film und Substrat aus. Es hat sich gezeigt, dass Tripellinien, an denen drei Körner angrenzen, verstärkt zur Lochbildung neigen [Sch00]. Energetische Betrachtungen zur Stabilität dieser komplexeren Strukturen wurden beispielsweise von Srolovitz und Safran durchgeführt [Sro86].

Treibende Kraft für die Ausbreitung der Löcher ist die Minimierung der Grenzflächenenergie. Wird der Einfachheit halber ein zylinderförmiges Loch mit Radius R in einem Film der Dicke d betrachtet, so ergibt sich aus Gleichung 6.1 eine Differenz der freien Energie von $\Delta \mathrm{F}=\pi \mathrm{R} \gamma_{\mathrm{Film}}(2 \mathrm{~d}+\mathrm{R}(\cos \theta-1))$. Diese Funktion besitzt bei $\mathrm{R}_{\mathrm{C}}$ ein Maximum, sodass ein kritischer Radius

$$
\frac{\mathrm{dF}}{\mathrm{dR}}\left(\mathrm{R}_{\mathrm{C}}\right)=0 \quad \Rightarrow \quad \mathrm{R}_{\mathrm{C}}=\frac{\mathrm{d}}{1-\cos \theta}
$$

existiert, oberhalb dessen die Löcher im Mittel wachsen, während kleinere Löcher instabil sind.

In einem Modell von Brandon und Bradshaw [Bra66] resultiert das auf Grund der konvexen Krümmung der entstehenden Randwulst erhöhte chemische Potential in einem Teilchenstrom in Richtung des ungestörten Films, der letztlich das Wachstum der Löcher hervorruft. Basierend auf Oberflächenselbstdiffusion als tragender Transportmechanismus liefert das Modell unter der Annahme eines $90^{\circ}$-Kontaktwinkels zwischen Film und Substrat und eines konstanten Krümmungsradius der Randwulst durch Lösen der NernstEinstein-Gleichung [Mul57] für die zeitliche Entwicklung des Lochradius:

$$
\frac{d R(t)}{d t}=\left(\frac{8 \pi \beta^{2}}{125 d^{3}}\right)^{1 / 5} t^{-3 / 5},
$$


wobei $\beta=\mathrm{D} \rho \Omega^{2} \gamma / \mathrm{k}_{\mathrm{B}} \mathrm{T}, \rho$ die Oberflächendichte der Atome, $\mathrm{D}$ den Oberflächendiffusionskoeffizienten, $\Omega$ das Atomvolumen, $\gamma$ die Oberflächenenergie und die Dicke des Films bezeichnet.

Jiran und Thompson [Jir90] haben, gestützt auf Beobachtungen entnetzender Goldschichten auf $\mathrm{SiO}_{2}$, eine laterale Instabilität des Filmrandes postuliert. Hierbei ist die Randwulst Dickenschwankungen unterworfen und die präferentielle Ausbreitung entnetzter Flächen in den Film an den Orten geringster Dicke führt zu einer komplexen Geometrie der Entnetzungsfront mit fingerarti-
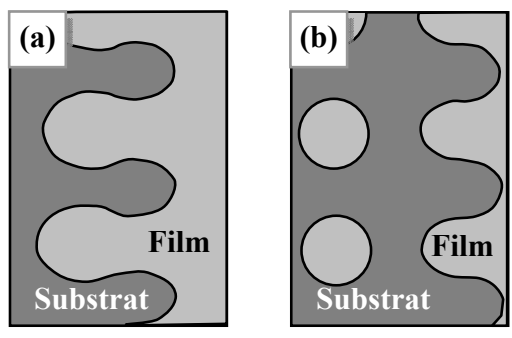

Abbildung 6.5: (a) Fingerartige Entwicklung der Entnetzungsfront und (b) Zerfall in isolierte Inseln. Nach [Jir90]. gen Strukturen, die im Zuge der Oberflächenminimierung in isolierte Inseln zerfallen (Abbildung 6.5). Entsprechende Rechnungen liefern eine zeitunabhängige Ausbreitungsgeschwindigkeit dx/dt der Entnetzungsfront

$$
\frac{\mathrm{dx}}{\mathrm{dt}}=\frac{2 \beta}{\pi \mathrm{d}^{3}}
$$

Die dargestellten Modelle berücksichtigen hingegen nicht die jeweilige Mikrostruktur des Films, und die kapillaritätsbasierten Konzepte können nur als stark vereinfachende Grundlage für ein Verständnis des Entnetzungsprozesses dienen. AFM-Untersuchungen von Klinger und Rabkin zeigen beispielsweise eine von Mullins Theorie deutlich abweichende Form der Furchen infolge der Oberflächenfacettierung [Kli01], welche aus der Anisotropie der Oberflächenenergie resultiert. Dem Gedanken folgend ist eine streng kreisförmige Ausbreitung der Löcher nicht a priori anzunehmen, wie entsprechende Untersuchungen zeigen [Ish03, Dor06, Bou07]. Zudem können mechanische Spannungen eine wichtige Rolle bei der Destabilisierung geschlossener Filme fester Phase einnehmen. Üblicherweise wird beobachtet, dass Zugspannungen durch Erhöhung der Korngrenzenenergie die Agglomeration fördern, wohingegen Druckspannungen durch Ausbildung von kleinen Hügeln auf der Filmoberfläche relaxieren und die Initialisierung des Entnetzungsprozesses hemmen, die Agglomeration jedoch nicht notwendigerweise unterbinden [Sha80].

Weiterhin haben Asaro, Tillert [Asa72] und Grinfeld [Gri86] eine spannungsinduzierte morphologische Instabilität beschrieben, die die Ausbildung zusätzlicher Grenzflächen begünstigt. Ausgehend von einer wellenförmig gestörten Oberfläche resultiert eine anliegende mechanische Spannung unabhängig von ihrem Vorzeichen (sei es nun eine Zugoder Druckspannung) in einer Spannungskonzentration in den Tälern und einer Spannungsrelaxation in den Hügeln [Gao91], da das Volumenmaterial geometrisch bedingt gewisser- 
maßen stärkeren Zwang auf die Täler ausübt ${ }^{16}$. Dieser Gradient der Verzerrungsenergie induziert einen Materialtransport, sodass die Hügel auf Kosten der Täler wachsen und folglich ein Wachstum der Störung hervorgerufen wird. Srolovitz [Sro89] hat mittels theoretischer Überlegungen die Instabilität anhand eines halbunendlichen Festkörpers untersucht und geschlossen, dass diese zur Inselbildung fester Filme führen kann. Die Anwendbarkeit der Resultate auf ein verspanntes Film-Substrat-System ist indes nicht klar auf Grund der Nichtberücksichtigung möglicher Unterschiede der elastischen Eigenschaften von Film und Substrat. Bekannt ist hingegen die Tatsache, dass dieser Effekt durch den Spannungszuwachs mit zunehmender Schichtdicke zum Stranski-Krastanov-Wachstum von zunächst epitaktisch wachsenden Filmen führen kann [Spe93].

\subsection{Entnetzungsverhalten von Nickel auf $\mathrm{SiO}_{2}$}

In diesem Abschnitt wird die temperaturabhängige Morphologieentwicklung sowie Kinetik dünner entnetzender Nickelschichten in fester Phase untersucht. Hierzu wurden bei Raumtemperatur mittels Elektronenstrahlverdampfens Nickelfilme einer Dicke von 5nm auf ebenes thermisch oxidiertes Silizium deponiert, in situ bei unterschiedlichen Temperaturen für eine Stunde ausgelagert und anschließend ex situ in einem REM untersucht. Dabei werden unterschiedliche Stadien des Entnetzungsprozesses beobachtet.

\subsubsection{Morphologieentwicklung}

Die bei Raumtemperatur deponierte Schicht weist einen zusammenhängenden Film auf, der das Substrat vollständig bedeckt (Abbildung 6.6(a)). Nun wachsen polykristalline Filme nicht atomar glatt auf, sondern bilden schon während des Depositionsprozesses dort, wo die Korngrenzen auf die freie Oberfläche treffen, Furchen aus. Anhand dieser Furchen lässt sich die Korngröße zu etwa 5 bis maximal 10nm abschätzen, sodass die Körngröße etwa in der Größenordnung der Schichtdicke liegt. Mittels AFM-Messungen (Anhang D) ergibt sich für diese Probe eine RMS-Rauigkeit von $\sigma_{\mathrm{RMS}}=0.23(1) \mathrm{nm}$. Bei höheren Temperaturen kommt es zur Nukleation von Löchern, wobei die größeren Löcher meist eine längliche Geometrie aufweisen und die kleineren Löcher eine nahezu kreisförmige Gestalt besitzen (Abbildung 6.6(b)). Obwohl die AFM-Spitze auf Grund ihres höheren Krümmungsradius nicht in die Löcher einzudringen vermag, ist über den Anstieg der Rauigkeit auf $\sigma_{\mathrm{RMS}}=$ 0.34(2)nm eine deutliche Änderung der Oberflächenstruktur zu messen. Während des Wachstums dieser Löcher wird ein zunehmender Anteil der Substratoberfläche freigelegt

\footnotetext{
${ }^{16}$ Dies wird intuitiv klar am Beispiel eines Gummibandes, dass eine oberflächliche Lamellenstruktur besitzt. Wird das Gummiband einer uniaxialen Zugspannung ausgesetzt, so weiten sich die Täler, während die Spitzen der Lamellen weitestgehend spannungsfrei bleiben.
} 

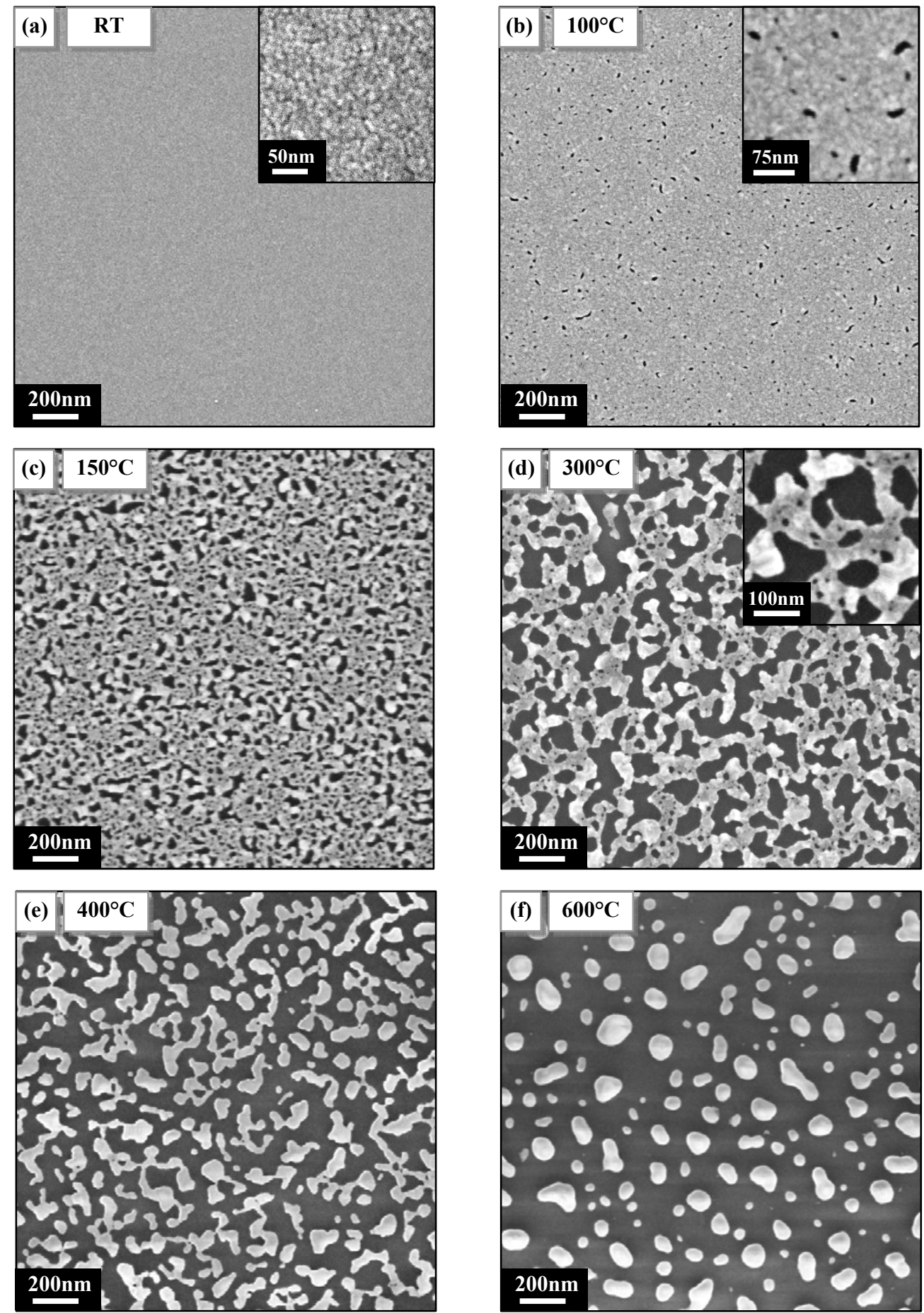

Abbildung 6.6: REM-Aufnahmen der bei unterschiedlichen Temperaturen ausgelagerten $5 \mathrm{~nm}$ Nickelschichten auf thermisch oxidiertem Silizium. 

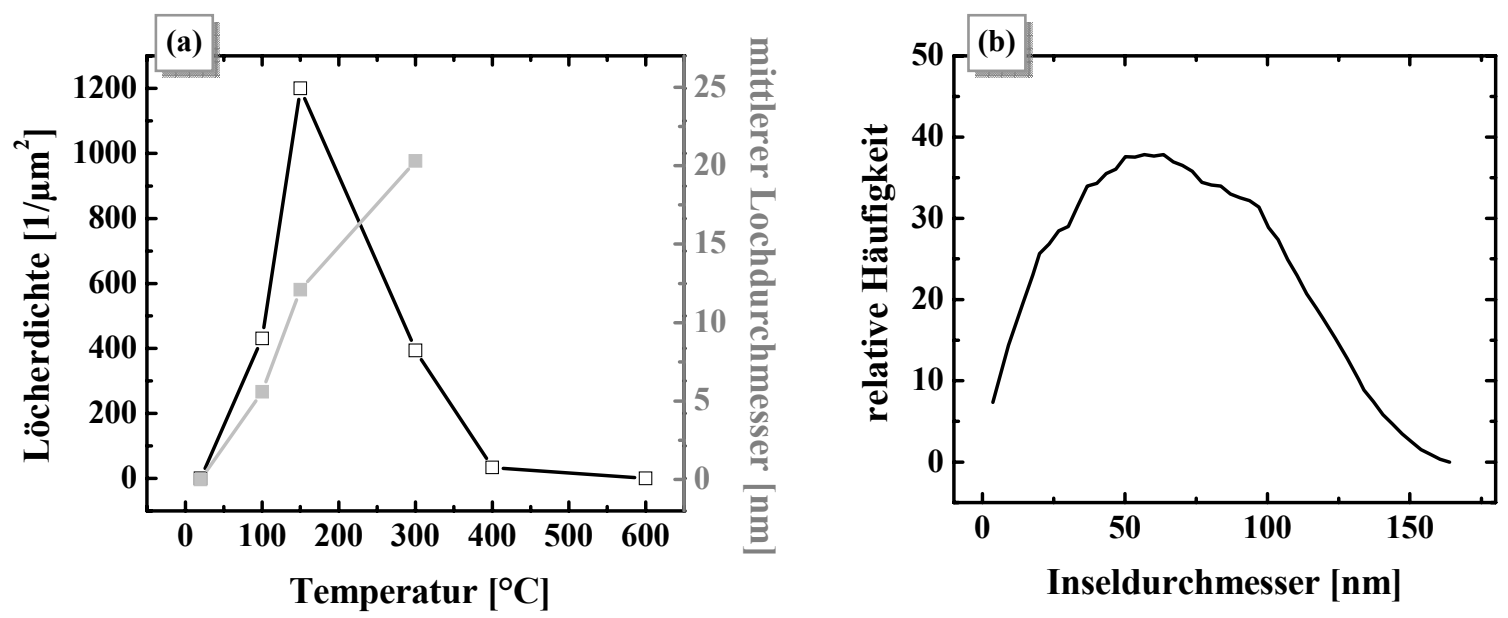

Abbildung 6.7: (a) Temperaturverlauf der Löcherdichte und des flächenäquivalenten mittleren Lochdurchmessers. (b) Inselgrößenverteilung der bei $600^{\circ} \mathrm{C}$ ausgelagerten Probe.

und das hierbei entfernte Schichtmaterial zwischen den entnetzten Gebieten aufgeschüttet (Abbildung 6.6(c)). Analog zur Bestimmung der Inselgrößenverteilung im vorherigen Kapitel wurden unter Verwendung eines Schwellenwertes bezüglich der Grauwertskala der REM-Aufnahmen die Löcher markiert und deren Anzahldichte sowie die flächenäquivalenten mittleren Lochdurchmesser errechnet (Abbildung 6.7(a)). Die Zunahme der Löcherdichte bei wachsendem Lochdurchmesser bis zu einer Auslagerungstemperatur von $150^{\circ} \mathrm{C}$ zeigt, dass mit steigender Temperatur die Anzahl der Nukleationspunkte wächst und die Phasen der Nukleation und des Wachstums nicht streng zu trennen sind.

$\mathrm{Ab}$ einer Auslagerungstemperatur von $300^{\circ} \mathrm{C}$ reduziert sich die Anzahl der Löcher bei zunehmendem Durchmesser durch den Prozess der Koaleszenz. Kurz vor dem Zusammenwachsen benachbarter entnetzter Bereiche, sind diese nur noch durch dünne Stege voneinander getrennt. Die Stege verbinden kompakte Strukturen mit einer lateralen Ausdehnung von bis zu 80nm, welche keine Löcher mehr aufweisen (Abbildung 6.6(d)). Durch das Zersetzen der Stege wachsen die kompakten Strukturen weiter an, der Film wird unterbrochen und in vorerst noch verzweigte isolierte Inseln (Abbildung 6.6(e)), anschließend in kompakte, nahezu kreisförmige Partikel mit einer breiten Größenverteilung (Abbildung 6.7(b)) und einem maximalen Durchmesser von etwa 150nm umgewandelt (Abbildung 6.6(f)). Der vergrößerte Bereich in Abbildung 6.6(d) zeigt exemplarisch das Abreißen eines Steges. Dabei bildet sich ähnlich wie bei der von Jiran und Thompson [Jir90] beobachteten fingerartigen Randinstabilität der zurückweichenden Entnetzungsfront an seinem Ende eine tropfenförmige Verdickung. 


\subsubsection{Kinetik des Entnetzungsprozesses}

Zur Untersuchung der Prozesskinetik wurden bei Raumtemperatur mittels Sputterdeposition $5 \mathrm{~nm}$ Nickelschichten auf thermisch oxidiertes Silizium deponiert und durch in situ Temperaturbehandlung unter Verwendung unterschiedlicher Heizraten über eine Vierpunktmessung der Temperaturverlauf des elektrischen Widerstands bestimmt. Die Messung des Widerstands während der Deposition erlaubt zudem eine Abschätzung der Schichtdicke, ab der sich durch Koaleszenz benachbarter Inseln geschlossene Strompfade bilden.

\subsubsection{Widerstandsverlauf während der Deposition}

Das Widerstandsverhalten dünner metallischer Filme wird durch eine Vielzahl von Ursachen elektronischer Streuung beeinflusst. Streuung resultiert aus der Wechselwirkung mit Phononen, Defekten, Verunreinigungen sowie Grenzflächen, zu denen auch Oberflächen und Korngrenzen zählen. Theoretische Überlegungen bezüglich der Schichtdickenabhängigkeit des Widerstandes speziell hinsichtlich mikrostruktureller Eigenschaften wie Korngröße und Oberflächenrauigkeit sind in [Jac90, Bar02] näher erläutert und gehen auf die grundlegenden Arbeiten von Fuchs und Sondheimer [Son52] sowie Mayadas und Shatzkes [May70] zurück. Der hier ermittelte spezifische Widerstand (Abbildung 6.8(a)) zeigt einen qualitativ ähnlichen Verlauf wie er beispielsweise von Barnat et al. [Bar02] beim Wachstum dünner Kupferschichten beobachtet wurde und beträgt für eine Schichtdicke von $5 \mathrm{~nm}$ mit $\rho_{\mathrm{R}}=1.0 \cdot 10^{-4} \Omega \mathrm{cm}$ etwa das 14-fache des Bulk-Wertes. Zur Bestimmung des Widerstandes während der Deposition wurde stark vereinfachend eine Parallelschaltung aus Plasma, das auf Grund der freien Ladungsträger ebenfalls zum Stromfluss beiträgt, und der Nickelschicht angenommen. Der Beitrag des Plasmas wurde dabei als konstant angesetzt. In Abbildung 6.8(b) ist die Entwicklung des Gesamtwiderstandes dargestellt. Zu Beginn des Sputterprozesses sinkt der Widerstand ${ }^{17}$ schlagartig infolge der Erzeugung freier Elektronen und der Ionisation von Argongas. Das Einsetzen des zweiten Widerstandsabfalls wird als Beginn der Koaleszenz isolierter Nickelinseln zu einem geschlossenen Film gedeutet und wird unter der Voraussetzung konstanter Depositionsrate bei einer zeitäquivalenten Schichtdicke von 1.7(2)nm beobachtet.

Nach Beendigung des Depositionsvorgangs bleibt der Widerstand zunächst nicht konstant, sondern zeigt eine langsame, aber kontinuierliche Abnahme (Abbildung 6.8(b)). Innerhalb der ersten drei Minuten wird ein Abfall von etwa 1.5\% gemessen. In diesem Zeitraum sinkt ebenfalls die Temperatur um $0.5^{\circ} \mathrm{C}$, welche zuvor durch den Energieeintrag während des Sputterprozesses um maximal $1{ }^{\circ} \mathrm{C}$ angestiegen ist. Die Widerstandsabnahme kann jedoch nicht allein auf die Temperaturabhängigkeit der Elektron-Phonon-Streuung

\footnotetext{
${ }^{17}$ Der endliche Widerstand vor Beginn des Depositionsvorgangs ist apparativ bedingt und resultiert aus der stromkontrollierten Messung bei begrenzter Maximalspannung.
} 

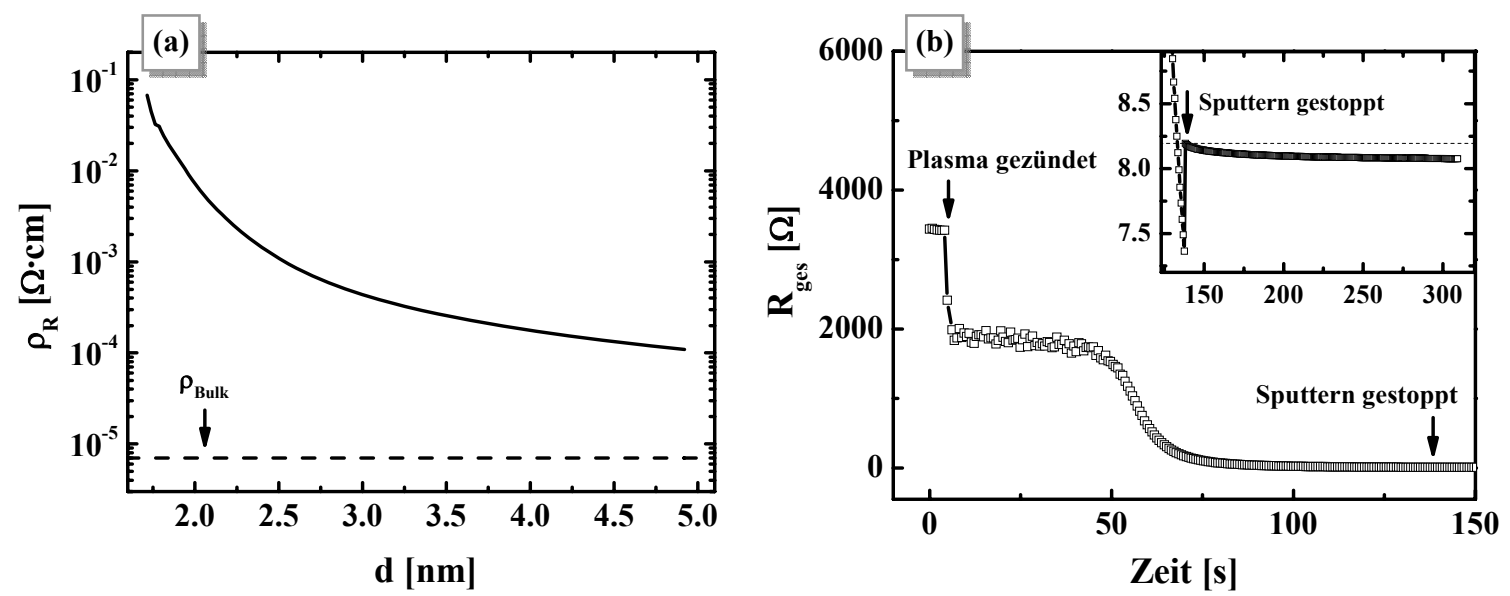

Abbildung 6.8: (a) Spezifischer Widerstand der Nickelschicht und (b) gemessener Gesamtwiderstand während und nach der Sputterdeposition.

und der Spinordnung zurückgeführt werden, die einen um nahezu eine Größenordnung geringeren Effekt mit sich bringen, wie der Temperaturkoeffizient der Widerstandsänderung einer 100nm dicken Nickelschicht nach thermischem Anlassen verdeutlicht (siehe Anhang F). Die Temperaturabhängigkeit der Oberflächen- und Korngrenzenstreuung ist ebenfalls deutlich geringer [Wu04]. Demzufolge sind bereits bei Raumtemperatur auftretende strukturelle Änderungen ursächlich. Hierbei sind Relaxationsprozesse durch das Ausheilen von Argoneinschlüssen, Punkt- und Liniendefekten einerseits sowie ein durch Minimierung von Korngrenzen- und Grenzflächenenergie getriebenes Kornwachstum andererseits möglich. Rossnagel und Kuan [Ros02] haben entsprechende Untersuchungen an gesputterten Kupferschichten durchgeführt und bei einer Schichtdicke von $4.5 \mathrm{~nm}$ eine Widerstandsabnahme von 50\% nach 1000 Minuten festgestellt. Anhand von XRD- und TEMMessungen konnten sie ein deutliches Kornwachstum sowie eine Rotation einzelner Körner nachweisen. Durch logarithmische Extrapolation des Kurvenverlaufs erweist sich die Widerstandsreduktion hier mit nur 2\% nach 1000 Minuten jedoch als erheblich geringer.

\subsubsection{Widerstandsverlauf während der Entnetzung}

Abbildung 6.9(a) zeigt den temperaturabhängigen Verlauf des elektrischen Widerstandes nach Deposition für unterschiedliche konstante Heizraten. Alle Kurven weisen ein qualitativ ähnliches Verhalten mit einer deutlichen Widerstandsreduktion gefolgt von einem rapiden Anstieg auf, der sich mit steigender Heizrate zu höheren Temperaturen verschiebt. Die genaue Charakteristik der Widerstandsentwicklung soll am Beispiel einer Heizrate von $10^{\circ} \mathrm{C}$ (Abbildung 6.9(b)) beschrieben werden.

Die Probe wurde in zwei Schritten aufgeheizt. Während des ersten Aufheizvorgangs bis $200^{\circ} \mathrm{C}$ wird eine kontinuierliche Widerstandsabnahme von bis zu $46 \%$ beobachtet. An- 

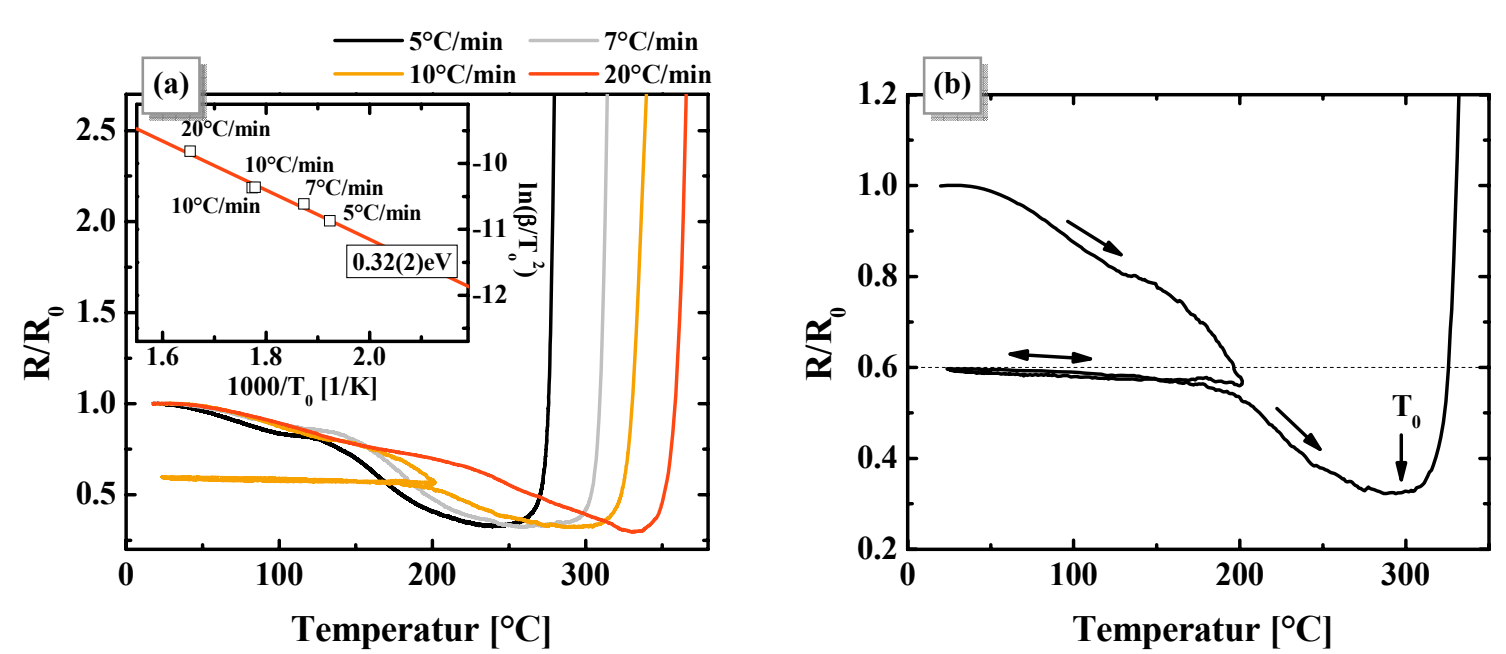

Abbildung 6.9: (a) Normierter Widerstand sputterdeponierter 5nm Ni-Filme bei unterschiedlichen Heizraten. Der eingesetzte Graph zeigt die Kissinger-Analyse der Übergangstemperaturen $\mathrm{T}_{0}$. (b) Normierter Widerstand bei einer Heizrate von $10^{\circ} \mathrm{C} / \mathrm{min}$. Bei Erreichen von $200^{\circ} \mathrm{C}$ wurde die Probe zunächst wieder abgekühlt.

schließendes Abkühlen auf Raumtemperatur führt zu einem nahezu linearen Anstieg um etwa 6\%. Dieser lineare Verlauf wird zu Beginn des zweiten Aufheizvorgangs zunächst reversibel durchlaufen, bevor es etwas unterhalb von $200^{\circ} \mathrm{C}$ zu einem steileren Abfall und nach Erreichen eines Minimums bei $\mathrm{T}_{0}=291^{\circ} \mathrm{C}$ zu einem rapiden Widerstandsanstieg über mehrere Größenordnungen kommt, der als Aufbrechen der Strompfade und Zerfall der Nickelschicht in isolierte Strukturen gedeutet werden kann. Insgesamt wird zwischen Raumtemperatur und $\mathrm{T}_{0}$ eine Widerstandsabnahme von $70 \%$ beobachtet, die größtenteils irreversibel und folglich auf strukturelle Änderungen zurückzuführen ist. Die Temperatur $\mathrm{T}_{0}$ des Widerstandsminimums wird indes nicht durch das zwischenzeitliche Abkühlen beeinflusst, wie entsprechende Messungen gezeigt haben. Weiterhin ist anzumerken, dass im Widerstandsabfall im Temperaturbereich unterhalb von $200^{\circ} \mathrm{C}$ eine Ausbuchtung mit konvexem Kurvenverlauf auftritt, die ebenfalls charakteristisch für alle Heizraten ist, und auf das Einsetzen eines weiteren Prozesses hindeutet.

Die Transformationstemperatur metastabiler Zustände, deren Umwandlung auf thermisch aktivierten kinetischen Prozessen beruhen, ist im Allgemeinen ratenabhängig. Diese Ratenabhängigkeit wird im Folgenden zur Bestimmung der Aktivierungsenergie $\Delta \mathrm{E}_{\mathrm{a}}$ herangezogen. Hierzu wurde die Kissinger-Gleichung [Kis56] verwendet, welche auf allgemeinen reaktionskinetischen Überlegungen und der von Arrhenius postulierten Beziehung für die Reaktionsrate K

$$
\mathrm{K}=\mathrm{K}_{0} \exp \left(-\frac{\Delta \mathrm{E}_{\mathrm{a}}}{\mathrm{k}_{\mathrm{B}} \mathrm{T}}\right)
$$


basiert. $\mathrm{K}_{0}$ ist eine Materialkonstante und wird als sogenannter Frequenzfaktor bezeichnet. Die Kissinger-Gleichung liefert für die Heizrate $\beta$ und Transformationstemperatur $T_{0}$ folgenden Zusammenhang ${ }^{18}$ :

$$
\ln \left(\frac{\beta}{\mathrm{T}_{0}^{2}}\right)=-\frac{\Delta \mathrm{E}_{\mathrm{a}}}{\mathrm{k}_{\mathrm{B}} \mathrm{T}_{0}}+\ln \left(\frac{\mathrm{K}_{0} \mathrm{k}_{\mathrm{B}}}{\Delta \mathrm{E}_{\mathrm{a}}}\right),
$$

Eine detaillierte Herleitung ist z.B. in [Bra04] zu finden. Mittels linearer Regression (Abbildung 6.9(a)) ergibt sich aus den für unterschiedliche Heizraten anhand des Widerstandsverlaufs ermittelten Transformationstemperaturen $\mathrm{T}_{0}(\beta)$ des Agglomerationsprozesses eine Aktivierungsenergie von $\Delta \mathrm{E}_{\mathrm{a}}=0.32(2) \mathrm{eV}$, welche den kinetisch limitierenden Prozess charakterisiert.

\subsubsection{Einfluss von Sauerstoff}

Auf Grund der entscheidenden Bedeutung der Grenzflächeneigenschaften während des Entnetzungsprozesses und des starken Einflusses von Verunreinigungen speziell auf die Grenzflächenenergien ist die Vermeidung von Oxidation durch in situ Messungen eine grundlegende Anforderung. Dies äußert sich unter anderem in einem deutlichen Anstieg des Widerstands an Luft. Der Einfluss von Sauerstoff auf den elektrischen Widerstand einer 5nm Ni-Schicht ist in Abbildung 6.10(a) dargestellt. Hierzu wurde der Kammerdruck nach Deposition durch kurzzeitiges Öffnen eines Nadelventils schlagartig auf $1 \cdot 10^{-2}$ mbar erhöht und der zeitliche Verlauf des Widerstands aufgezeichnet.

Experimentelle Untersuchungen an metallischen Schichten haben insbesondere bezüglich der Oxidschichtdicke unterschiedliche Gesetzmäßigkeiten der Oxidationskinetik aufgezeigt [Ade92]. Das hier beobachtete Verhalten zeigt gute Konformität mit der CabreraMott-Theorie für sehr dünne Schichten [Atk85]. In dieser Theorie wird der Transport von Sauerstoff- bzw. Metallionen durch das elektrische Feld getrieben, welches durch den Elektronentransfer von der metallischen Schicht zu adsorbierten Sauerstoffatomen entsteht. Dabei wird angenommen, dass stets ein Ausgleich des elektrochemischen Potentials zwischen Metall und Adsorbatschicht stattfindet, was für sehr dünne und somit tunnelnde Oxidschichten plausibel erscheint. Die Kinetik führt zur zeitlichen Entwicklung der Oxiddicke

$$
x(t)=a_{1} \ln \left(a_{2} t+a_{3}\right),
$$

\footnotetext{
${ }^{18}$ Streng genommen ist hier die Temperatur der maximalen Transformationsrate zu verwenden, wobei diese häufig nicht zugänglich ist und näherungsweise durch die sogenannte Onset-Temperatur des Transformationsbeginns substituiert wird.
} 

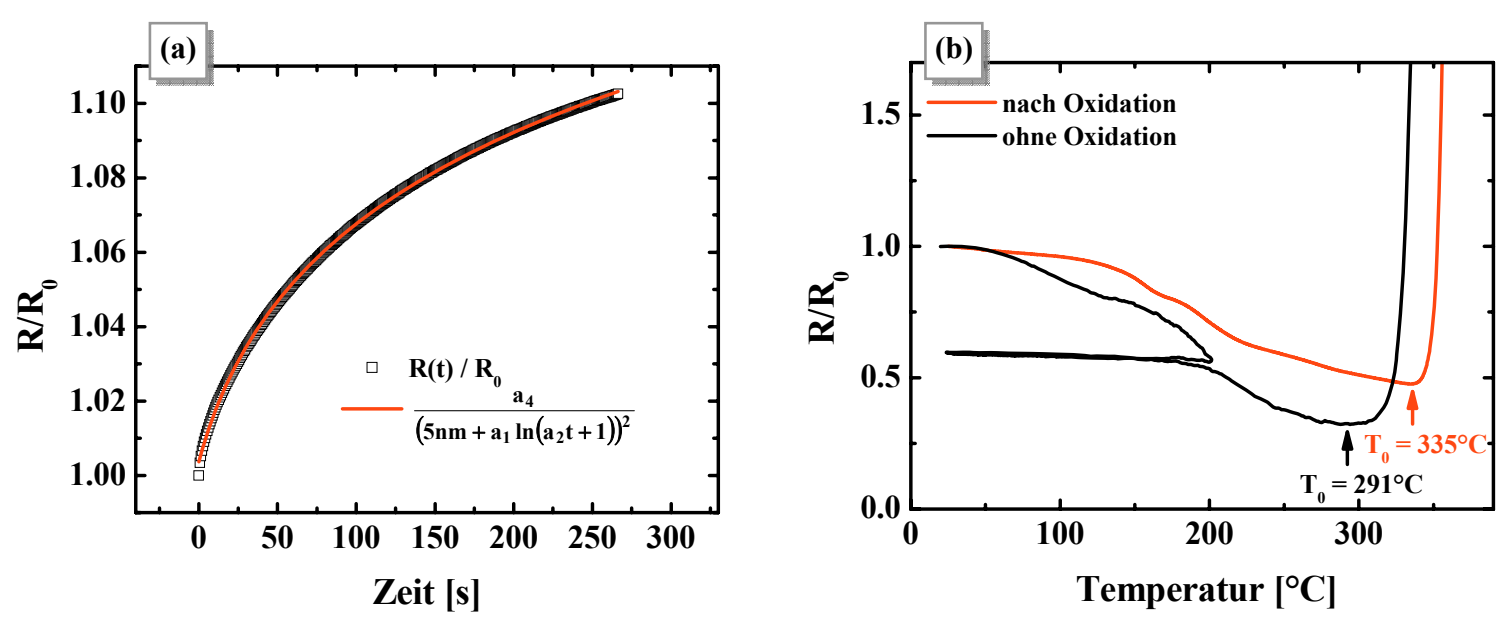

Abbildung 6.10: (a) Widerstandsverlauf einer 5nm Nickelschicht während Oxidation. (b) Temperaturabhängiger Widerstandsverlauf der oxidierten Schicht und einer nicht oxidierten Schicht bei einer Heizrate von $10^{\circ} \mathrm{C} / \mathrm{min}$.

wobei $a_{1}, a_{2}$ und $a_{3}$ Konstanten sind [Atk85]. Wird nun davon ausgegangen, dass das Oxid nur unwesentlich zum Stromfluss beiträgt (was nicht ganz der Wahrheit entspricht, da Nickeloxid einen halbleitenden Charakter besitzt), lässt sich eine effektive Schichtdicke $\mathrm{d}_{\text {eff }}=\mathrm{d}_{0}-\mathrm{x}$ definieren, wobei die Fuchs-Sondheimer-Theorie durch die mit abnehmender Schichtdicke erhöhte Oberflächenstreuung näherungsweise eine $\rho_{R} \propto 1 / d_{\text {eff }}$-Abhängigkeit liefert. Somit lässt sich für den elektrischen Widerstand über die Beziehung $R \propto \rho_{R} / d$ und damit $\mathrm{R} / \mathrm{R}_{0} \propto \rho_{\mathrm{R}} / \mathrm{d}_{\text {eff }} \propto\left(\mathrm{d}_{0}-\mathrm{x}\right)^{-2}$ folgende Gesetzmäßigkeit ableiten:

$$
\mathrm{R} / \mathrm{R}_{0}=\mathrm{a}_{4}\left(\mathrm{~d}_{0}+\mathrm{a}_{1} \ln \left(\mathrm{a}_{2} \mathrm{t}+\mathrm{a}_{3}\right)\right)^{-2},
$$

wobei aus der Bedingung, dass vor Öffnen des Nadelventils keine Oxidation stattgefunden hat, aus Gleichung $6.9 \mathrm{a}_{3}=1$ folgt. Eine Anpassung des Kurvenverlaufs basierend auf Gleichung 6.10 zeigt gute Übereinstimmung und erlaubt durch Einsetzen der deponierten Schichtdicke von $\mathrm{d}_{0}=5 \mathrm{~nm}$ eine Abschätzung der Oxidschichtdicke, sodass sich nach einer Oxidationsdauer von insgesamt $20 \mathrm{~h}$ eine Oxiddicke von etwa $\mathrm{x}=0.8 \mathrm{~nm}$ ergibt, was etwa zwei Monolagen entspricht.

Die für $20 \mathrm{~h}$ oxidierte Probe wurde anschließend bei einem Druck von $5 \cdot 10^{-7}$ mbar mit $10^{\circ} \mathrm{C} / \mathrm{min}$ auf $400^{\circ} \mathrm{C}$ aufgeheizt. Die temperaturabhängige Entwicklung des Widerstands lässt im Vergleich zur nicht oxidierten Nickelschicht ein qualitativ ähnliches Verhalten erkennen (Abbildung 6.10(b)). Auch mit oberflächlicher Oxidschicht wird bei steigender Temperatur eine anfängliche Widerstandsabnahme beobachtet. Ein ähnlich steiler Abfall wie bei reinen Nickelschichten sowie die Transformationstemperatur des Agglomerationsprozesses sind indes $\mathrm{zu}$ höheren Temperaturen verschoben. Insgesamt fällt die Widerstandsabnahme mit maximal 50\% merklich geringer aus. 


\subsubsection{Diskussion}

\subsubsection{Morphologie}

Nukleation von Löchern: Mullins [Mul58] hat postuliert, dass Kornwachstum weitestgehend durch die Furchenbildung unterdrückt wird, wenn die Korndurchmesser der Schichtdicke entsprechen oder diese übersteigen. Dies konnte beispielsweise anhand der Agglomeration dünner Silberfilme auf SiN experimentell bestätigt werden [Dan00]. Obwohl die unbehandelte Nickelschicht mit einer Dicke von 5nm und einer Korngröße von ebenfalls etwa $5 \mathrm{~nm}$ diese Bedingung erfüllt, kann das Einsetzen von Kornwachstum bereits während des Frühstadiums der Entnetzung hier nicht vollständig ausgeschlossen werden, wie die Widerstandsabnahme mit dem zeitlichen Verlauf nach der Deposition bei Raumtemperatur (Abbildung 6.8(b)) andeutet, sodass zu einer genauen Klärung weiterführende Untersuchungen erforderlich sind.

Die Entnetzung dünner fester Filme ist ein komplexer Prozess, dessen kinetischer Pfad und resultierende Strukturbildung von einer Vielzahl von Parametern beeinflusst wird. Das grundsätzliche Verhalten entspricht insoweit dem eines isotropen Flüssigkeitsfilms, als es im Wesentlichen durch die auftretenden Grenzflächenenergien bestimmt wird. Es herrscht allgemeiner Konsens in der Annahme, dass das Entnetzen polykristalliner Filme durch Lochbildung initi-

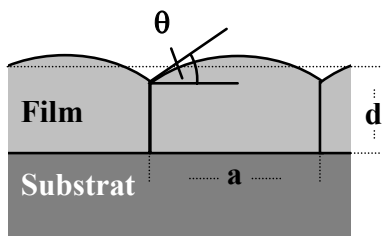

Abbildung 6.11: Geometrische Gleichgewichtskonfiguration durch Furchenbildung an Korngrenzen nach [Sro86]. iert wird, welche vornehmlich aus der Furchenbildung an Korngrenzen und Korngrenzentripellinien hervorgeht [Mu157, Sro95]. Srolovitz und Safran [Sro86] haben diesbezüglich einen Zusammenhang zwischen Schichtstabilität und dem Verhältnis aus Korngröße zur Schichtdicke hergeleitet. Sie gehen hierbei von einer kolumnaren Kornstruktur aus (Abbildung 6.11), deren Kornoberfläche zur Minimierung der Oberflächenenergie in einer perfekten Kugelkappe resultiert und an der Korngrenze den Gleichgewichtswinkel nach Gleichung 6.3 besitzt. Aus der Volumenerhaltung und geometrischen Überlegungen wirkt hiernach die Furchenbildung destabilisierend, dergestalt, dass ihre Tiefe die Schichtdicke erreicht, wenn folgende Bedingung erfüllt ist:

$$
\frac{\text { Korndurchmesser }}{\text { Schichtdicke }}=\frac{\mathrm{a}}{\mathrm{d}}>\frac{6 \sin ^{3} \theta}{2-3 \cos \theta+\cos ^{3} \theta}
$$

Diese Bedingung lässt sich für die hier untersuchten Schichten genauer auswerten. Aus der Annahme, dass die Korngrenzenenergie die Oberflächenspannung nicht übersteigt, $\gamma_{\mathrm{Gb}}<\gamma_{\mathrm{FV}}$, folgt über Gleichung 6.3 für den Winkel $\theta<\pi / 6$ und aus der oben genannten 
Beziehung, deren rechter Term eine monoton fallende Funktion von $\theta$ darstellt, somit a $/ d>15$. Wird der Ansatz zusätzlich um eine Furchenbildung von der Film-SubstratGrenzfläche ausgehend erweitert, wie sie beispielsweise von Nolan et al. [No191] und Xiao et al. [Xia91] beobachtet wurde, halbiert sich die stabile Schichtdicke in etwa, da für die Grenzflächenenergie inerter Systeme eine analoge Annahme mit $\gamma_{\mathrm{Gb}}<\gamma_{\mathrm{FS}}$ legitimiert werden kann, sodass a/d $>7.5$. Aus der Abschätzung der Korndurchmesser mit bis zu $10 \mathrm{~nm}$ ergibt sich mit der Schichtdicke von 5nm jedoch maximal ein Verhältnis von a/d = 2, was demnach eine Nukleation von Löchern an planaren Korngrenzen ausschließt und diesbezüglich den Korngrenzentripellinien eine verstärkte Bedeutung zukommen lässt.

Einschränkend sollte hier erwähnt werden, dass dieser Ansatz auf rein kapillaritätstheoretischen Überlegungen beruht. Zudem können mechanische Spannungen die Korngrenzenenergie in erheblichem Maße beeinflussen und entsprechende Relaxation beispielsweise die Bedingung der Volumenerhaltung untergraben. Das angeführte Kriterium kann daher nur eine Abschätzung liefern.

Prozesse des Entnetzungsvorgangs: Die hier angestellten Untersuchungen zeigen überdies eine im Anfangsstadium vermehrt längliche Geometrie der Löcher. Dies wird im Rahmen einer phänomenologischen Modellvorstellung in Abbildung 6.12(a) verdeutlicht, die in idealisierter Weise die unterschiedlichen beobachteten Prozesse des Entnetzungsvorgangs darstellt. Die Koaleszenz benachbarter Löcher und das präferentielle Wachstum entlang der Korngrenzen unterminiert dabei eine kreisförmige Gestalt, welche aus der Minimierung des Umfangs auf Grund von Kapillarkräften in isotropen Medien folgen würde.

Während des Wachstums eines Lochs wird Material aus seinem Inneren entfernt, die Dreiphasengrenze bewegt sich auf den nicht entnetzten Film zu und die Oberflächenbedeckung nimmt ab (Abbildung 6.12(b)). Dabei entstehen Löcher, deren Dimension weit über die Abmessung einzelner Körner hinausgehen, sodass die Ausbreitung nicht mehr auf die Korngrenzen beschränkt ist. Die Zunahme der Rauigkeit nach der Nukleationsphase bei einer Auslagerungstemperatur von $100^{\circ} \mathrm{C}$ indiziert, dass das Material aus den Löchern nicht gleichmäßig auf dem Film verteilt wird, und deutet auf die in Analogie zu flüssigen Filmen auch für die Entnetzung fester Filme beobachtete Ausbildung von Randwülsten oder Dämmen [Jir90, Won00], auch wenn die Strukturen hier nicht im Einzelnen aufgelöst werden können und ein Kornwachstum nicht explizit ausgeschlossen wird. Die Anisotropie der Grenzflächenenergien bestimmt indes das Wachstumsverhalten einzelner Körner im Randbereich bei der Anlagerung des überschüssigen Materials und verhindert auch durch den begleitenden Effekt der Facettierung die Ausbildung eines streng toroidalen Randes.

Werden zwei dieser Dämme durch das Wachstum benachbarter Löcher zusammen geschoben, so entsteht eine halsähnliche Struktur (Abbildung 6.12(b)). Bei $300^{\circ} \mathrm{C}$ Auslagerungstemperatur ist die Lochdichte infolge von Koaleszenz merklich reduziert und die Ausbildung großer kompakter Strukturen ohne Löcher weist darauf hin, dass die zylindrischen 
(a) Lochnukleation

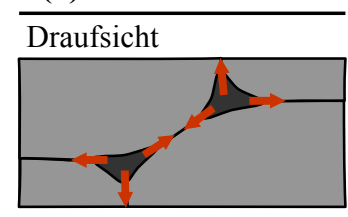

Querschnitt

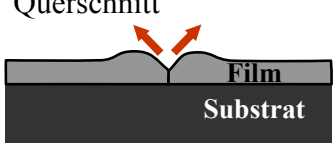

(b) Lochwachstum
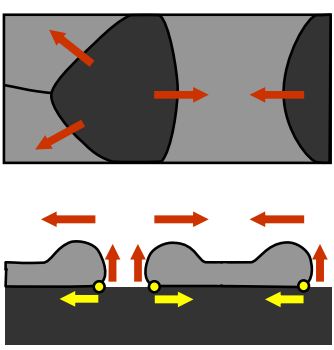
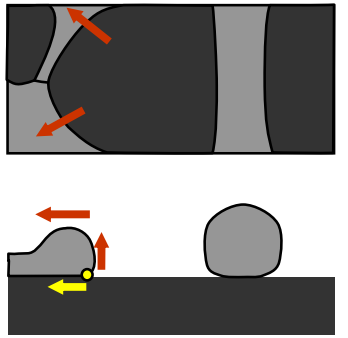

(c) Koaleszenz
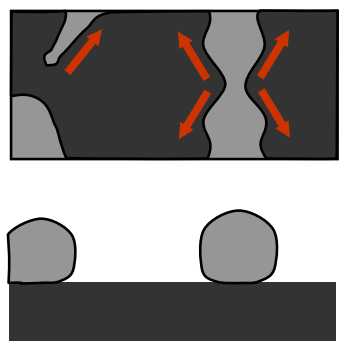

Abbildung 6.12: Modellhafte Beschreibung der morphologischen Entwicklung während der unterschiedlichen Entnetzungsphasen. Die roten Pfeile beschreiben die Bewegung von Schichtatomen, die gelben die Bewegung der Dreiphasengrenze.

Hälse nicht nur ausdünnen und aufbrechen, sondern auf Grund von Diffusionsprozessen, getrieben durch konkave Oberflächenkrümmung, auch wachsen können, wodurch eine Art Sintereffekt entsteht. Zum Aufbrechen der Hälse kann es einerseits an den instabilen Korngrenzen kommen, andererseits ist eine Art Rayleigh-Instabilität [Ray78] möglich (Abbildung 6.12(c)), wie sie beispielsweise bei der Fragmentierung dünner KupferNanodrähte beobachtet wurde [Mol04]. Dies führt letztlich zum Zersetzen der Schicht in isolierte Inseln. Die morphologische Entwicklung ist indes nach Ausbildung kompakter isolierter Strukturen nicht vollständig terminiert. Eine Auslagerung bei höheren Temperaturen kann die Größenverteilung der Inseln durch Ostwaldreifung [Ohr02] von größeren Inseln auf Kosten kleinerer zu einem gewissen Grad weiter modifizieren. Dieser Effekt ist diffusionsbasiert und erfordert je nach Temperatur im Allgemeinen geringe Partikelabstände.

\subsubsection{Widerstandsverhalten und Kinetik}

Widerstandssignaturen: Zunächst soll darauf hingewiesen werden, dass die ermittelten Widerstandscharakteristika nicht direkt mit den Untersuchungen der oberflächenmorphologischen Entwicklung in Abbildung 6.6 assoziiert werden können. Die aufgedampften Proben wurden für eine Stunde bei einer festen Temperatur gehalten, wohingegen die sputterdeponierten Schichten zur Widerstandsmessung mit konstanter Heizrate erwärmt wurden, sodass die Auswirkungen thermisch aktivierter Prozesse zu höheren Temperaturen verschoben sind. Andererseits zeigen sputterdeponierte Filme typischerweise eine höhere Defektkonzentration und eine veränderte Mikrostruktur [Tho77] mit größeren Korndurchmessern als Folge der höheren kinetischen Energie auftreffender Adatome. Dies führt zu einer geringeren thermischen Stabilität und für kleine Heiraten zum elektrischen Versagen bereits deutlich unterhalb von $300^{\circ} \mathrm{C}$. 
Der Verlauf des elektrischen Widerstands vor und während des Entnetzungsvorgangs ermöglicht in Ergänzung zu den morphologischen Untersuchungen ein genaueres Verständnis hinsichtlich struktureller Prozesse sowie der Prozesskinetik. Auf der Basis reiner Elektron-Phonon-Streuung wird ein linearer Anstieg des Widerstands mit der Temperatur erwartet. Die zeitliche Entwicklung bei Raumtemperatur zeigt hingegen, dass bereits nach der Deposition widerstandsreduzierende Relaxationsprozesse stattfinden. Der irreversible Anteil der temperaturabhängigen Wider-

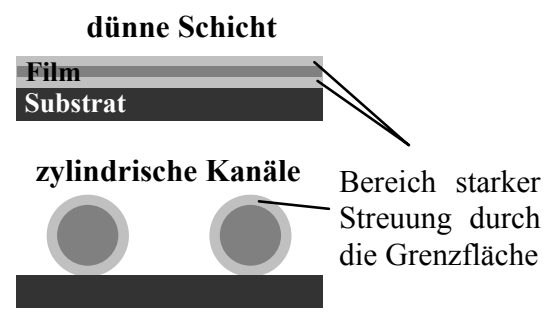

Abbildung 6.13: Stark vereinfachte Darstellung reduzierten Grenzflächeneinflusses durch Bildung zylindrischer Kanäle. standsabnahme kann zum einen auf thermisches Ausheilen von Defekten wie Leerstellenagglomeration und Austritt von Argoneinschlüssen durch die Filmoberfläche zurückgeführt werden, welche vom Sputtervorgang herrühren. Zum anderen führt das Wachstum der Löcher durch den Materialtransport und dem damit verbundenen Wachstum angrenzender Körner zur Ausbildung elektronischer Pfade geringerer Korngrenzendichte, wobei das Zusammenschieben zweier Dämme durch die Ausbreitung benachbarter Löcher die Formung von Kanälen oder Hälsen bewirkt (Abbildung 6.12(b)), deren Durchmesser die ursprüngliche Schichtdicke übersteigt, wie AFM-Messungen belegen. Dies minimiert das lokale Grenzflächen-zu-Volumen-Verhältnis des effektiven Filmquerschnitts, was eben gerade der ursprünglichen treibenden Kraft der Entnetzung, reduziert auf zwei Dimensionen, entspricht, und vermindert somit den Einfluss der Oberfläche (Abbildung 6.13), der sich auf Grund elektronischer Streuung nach der Fuchs-Sondheimer-Theorie [Son52] auf die freie Weglänge der Elektronen auswirkt. Folglich kann dieser Effekt trotz der erhöhten Anzahl an Störstellen in Form von isolierenden Löchern zu einer Reduzierung des Widerstands führen. Die letzte Phase der Entnetzung besteht schließlich in der Bildung von Verengungen entlang der Kanäle und dem Aufbrechen dieser halsartigen Strukturen, was einen rapiden Anstieg des Widerstands hervorruft und bis zum elektrischen Versagen der Schicht führt.

Für die nahezu linear verlaufende reversible Widerstandsabnahme mit steigender Temperatur während des zweiten Aufheizvorgangs kann aus mangelnder Kenntnis der genauen physikalischen Ursache nur eine spekulative Erklärung angegeben werden. Möglich ist hierbei ein Effekt der stark unterschiedlichen thermischen Ausdehnungskoeffizienten von Schicht und Substrat, welcher mit steigender Temperatur eine reversible Entwicklung von Druckspannungen in der Nickelschicht vermittelt. Diese Spannungen können die Korngrenzenenergie und somit die Tiefe der Furchen zwischen benachbarten Körnern modifizieren, wobei Heizen zu einer Abnahme und Kühlen zu einem Anstieg führt (Abbildung 6.14) und der elektrische Widerstand auf Grund unterschiedlicher Konstriktion des effektiven Leitungsquerschnitts in den Furchen reversibel variiert. Während des ersten Heizvorgangs wird dieser Effekt von irreversibler Furchenbildung infolge von atomarem Transport 
überlagert. In dünnen metallischen Schichten entstehende Druckspannungen werden häufig als Ursache für die Bildung von Hügeln, sogenannten Hillocks, herangezogen [Pen69]. Lahiri und Wells [Lah69] haben bei thermischer Behandlung von Aluminiumschichten auf $\mathrm{SiO}_{2}$ eine reversible Bildung von Hügeln beobachtet, die sie auf die Veränderung der mechanischen Spannung in der Schicht, verursacht durch unterschiedliche thermische Ausdehnungskoeffizienten, zurückführen.

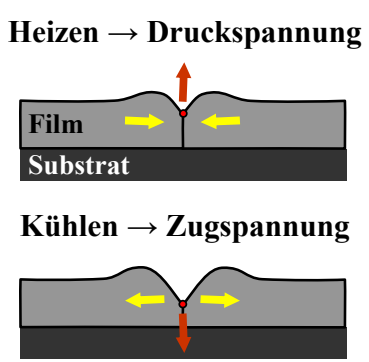

Abbildung 6.14: Einfluss mechanischer Spannungen auf die Furchentiefe.

Die instantan einsetzende Widerstandsabnahme von bis zu $70 \%$ bei Temperaturerhöhung wurde, soweit hier bekannt, an keinem anderen System beobachtet. Entsprechende Untersuchungen beschränkten sich bislang weitestgehend auf deutlich größere Schichtdicken [Sie01, Kim02]. Saxena et al. [Sax05] fanden an einer 20nm dicken Kupferschicht nach anfänglicher Widerstandszunahme einen geringen Einbruch während des Entnetzungsvorgangs, der sich auf einen schmalen Temperaturbereich beschränkt, wohingegen dickere Kupferfilme diesen Effekt nicht aufwiesen. Die Messungen wurden jedoch nicht in situ durchgeführt, sodass eine Bewertung in Anbetracht eines möglichen Einflusses von Verunreinigungen und Oxidation schwierig ist. Dennoch deuten die Resultate auf einen schichtdickenabhängigen Effekt hin.

Atomarer Transportmechanismus: Die Widerstandssignaturen können weiterhin Aufschluss über die vorherrschenden Transportmechanismen geben. Hierbei sind unterschiedliche Prozesse denkbar. Viskoses Fließen kann bei Temperaturen $\mathrm{T}<0.4 \mathrm{~T}_{\mathrm{m}}$ deutlich unterhalb der Schmelztemperatur des Bulk-Wertes trotz des Größeneffekts der Schmelzpunkterniedrigung für Nanostrukturen ausgeschlossen werden [Zha00]. Ähnliches gilt für einen signifikanten Einfluss von Verdampfen und Kondensation des Schichtmaterials auf die Morphologie innerhalb der hier betrachteten Zeitskala von rund einer Stunde [Mul57]. Demnach beschränkt sich der atomare Transport im Wesentlichen auf Diffusionsprozesse.

Die aus der Ratenabhängigkeit der Transformationstemperatur ermittelte Aktivierungsenergie von $\Delta \mathrm{E}_{\mathrm{a}}=0.32(2) \mathrm{eV}$ zeigt eine bemerkenswerte Übereinstimmung mit dem von Tung und Graham [Tun80] bestimmten Wert für Nickeloberflächendiffusion auf Ni(111)Ebenen von $0.33 \mathrm{eV}$ und indiziert, dass Oberflächendiffusion auf eben diesen Ebenen, welche auf Grund der fcc-Struktur die geringste Oberflächenenergie besitzen und somit präferentiell ausgebildet werden, den kinetisch limitierenden Prozess darstellt. Volumen- und Grenzflächendiffusion besitzen nicht allein infolge erhöhter Koordination der betreffenden Atome weitaus größere Aktivierungsenergien. Dennoch sind die bekannten meist phänomenologischen Modelle sowie experimentellen Daten diesbezüglich nicht eindeutig. Saxena et al. [Sax05] haben, basierend auf ähnlichen Experimenten zur Bestimmung der Akti- 
vierungsenergie für Kupfer auf $\mathrm{SiO}_{2}$, ein Modell vorgeschlagen, welches die Grenzflächendiffusion zwischen Schicht und Substrat als kinetisch limitierenden Prozess beinhaltet. Wie bereits erwähnt, sind die betreffenden Untersuchungen jedoch ohne Ausschluss von Oxidation durchgeführt worden. Eriksson et al. [Eri99] haben hingegen mittels kapazitiver Messungen an Palladium auf thermisch oxidiertem Silizium eine Aktivierungsenergie ermittelt, die mit Palladiumoberflächenselbstdiffusion übereinstimmt und somit der hier beobachteten Prozesskinetik entspricht.

Obwohl sich keine Signifikanz in der Heizratenabhängigkeit der konvexen Ausbuchtung des anfänglichen Widerstandsabfalls abzeichnet, suggeriert diese, dass entweder mehr als ein widerstandsreduzierender Relaxationsprozess oder ein entgegenwirkender Effekt mit abweichender Temperaturabhängigkeit auftritt. Dies unterstützt die Annahme einer zweiphasigen Widerstandsreduktion durch Defektrelaxation bei niedrigen Temperaturen und der Ausbildung elektronischer Pfade erhöhter Leitfähigkeit nach Nukleation von Löchern im Temperaturbereich oberhalb von $100^{\circ} \mathrm{C}$.

Einfluss von Sauerstoff und thermische Stabilität: Metalloxide besitzen auf Grund hoher Bildungsenthalpien im Allgemeinen eine erhöhte thermische Stabilität. Der Entnetzungsprozess der Nickelschicht wird durch die oberflächliche Oxidschicht auf Grund reduzierter Oberflächendiffusion kinetisch eingeschränkt, bis es vermutlich durch mechanische Spannungen, induziert durch unterschiedliche thermische Ausdehnung, sowie Diffusion des Metalls auf die Oberfläche zum Aufreißen der Oxidhaut kommt, sodass das elektrische Versagen zu höheren Temperaturen verschoben wird. Die oberflächliche Oxidation kann somit als einfaches Verfahren zur Verbesserung thermischer Stabilität metallischer dünner Schichten genutzt werden. Dies ist jedoch nur von technologischem Interesse, wenn die Oxidschicht wie etwa bei Aluminium passiviert und nicht in den gesamten Film diffundiert. Ein vergleichbarer Effekt wird durch gezielte Deposition einer (oxidischen) Deckschicht auf das Metall erreicht [Gad05]. Andererseits kann eine Oxidation auch zur Destabilisierung von Korngrenzen führen, wodurch der Agglomerationsprozess bei niedrigerer Temperatur initiiert wird, wie es beispielsweise an dünnen Palladiumschichten beobachtet wurde [Eri99].

\subsubsection{Wetting-Layer}

Es ist nicht auszuschließen, dass ein wenige Monolagen dicker Nickelfilm, ein sogenanntes Wetting-Layer, auf der Substratoberfläche zurückbleibt und damit das Entnetzungsverhalten über die veränderte Gleichgewichtsbedingung des Benetzungswinkels an der Tripellinie signifikant beeinflusst. Die Vermutung der Existenz eines Wetting-Layers gründet sich hier auf Untersuchungen von Entani et al. [Ent05], die ein Stranski-Krastanov-Wachstum dünner Kobaltfilme auf $\mathrm{SiO}_{2}$ beobachten konnten, wobei zunächst eine geschlossene Monolage 

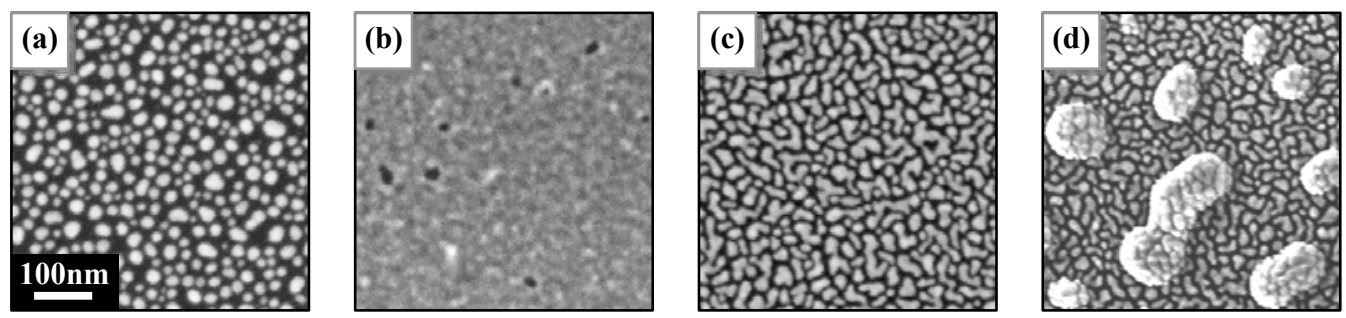

Abbildung 6.15: REM-Aufnahmen von bei Raumtemperatur deponierten $5 \mathrm{~nm}$ Ag-Schichten auf (a) $\mathrm{SiO}_{2}$, (b) $5 \mathrm{~nm}$ Ni-Schicht, (c) an Luft oxidierter $5 \mathrm{~nm} \mathrm{Ni-}$ Schicht und (d) bei $600^{\circ} \mathrm{C}$ ausgelagerter und an Luft oxidierter $5 \mathrm{~nm}$ Ni-Schicht. Der Maßstab aller Bilder ist identisch.

entsteht, auf der im weiteren Verlauf der Deposition Inselwachstum stattfindet. Diese Monolage zeigt keine ferromagnetische Ordnung und besitzt auf Grund der Tendenz metallischer Filme, auf oxidischen Substraten an der Grenzfläche zu oxidieren [Oh101, Che02], vermutlich oxidischen Charakter und somit eine starke Bindung zum Substrat.

Infolge der hohen Affinität des Nickels zu Sauerstoff wäre eine entsprechende Lage thermisch vergleichsweise stabil und auch hier zu vermuten. In TEM-Untersuchungen konnte jedoch kein Anzeichen für eine remanente Nickellage auf der Substratoberfläche nach dem Entnetzungsvorgang gefunden werden (siehe Anhang G). Dass dennoch auf ein zurückbleibendes Wetting-Layer geschlossen werden kann, lässt sich aus dem Nachweis über das Wachstumsverhalten einer nachträglich deponierten Silberschicht auf den entnetzten Nickelfilm folgern. Silber bildet nahezu kreisförmige isolierte Strukturen auf $\mathrm{SiO}_{2}$ (Abbildung 6.15(a)), eine bedeckende Schicht mit vereinzelten Löchern auf Nickel (Abbildung 6.15(b)) und eher längliche und verzweigte isolierte Strukturen auf oxidiertem Nickel (Abbildung 6.15(c)). Die Morphologie der Silberschicht in den Bereichen zwischen den Inseln einer durch thermische Auslagerung bei $600^{\circ} \mathrm{C}$ agglomerierten und anschließend an Luft oxidierten Nickelschicht (Abbildung 6.15(d)) stimmt jedoch nicht mit dem Verhalten auf reinem $\mathrm{SiO}_{2}$ überein, sondern ähnelt vielmehr der Gestalt des auf Nickeloxid deponierten Films, und stellt somit ein deutliches Anzeichen für eine während des Entnetzungsvorgangs auf dem Substrat zurückbleibende thermisch stabile Nickelschicht dar. Die Entnetzung findet folglich auf einem vermutlich nur eine Monolage dicken und über die Bindung zum Substrat oxidischen Wetting-Layer statt.

\subsection{Entnetzung von Nickel auf Rippelstrukturen}

Experimentelle und theoretische Untersuchungen der Instabilität, Dynamik und Morphologie dünner Filme bezüglich der Entnetzung beschränkten sich bislang im Wesentlichen auf chemisch homogene ebene Substrate. Verstärkt wird aktuell in Hinblick auf technologische Anwendungen versucht, durch Einbringen definierter Inhomogenitäten das Entnetzungs- 
verhalten gezielt zu steuern [Gau99, Die05, Moc05], wobei nicht allein auf Grund der Komplexität fester Filme zunächst flüssige Filme im Vordergrund standen. Im Folgenden soll der Einfluss topographischer Modifikationen am Beispiel einer Rippelstruktur untersucht werden, wobei speziell die Frage adressiert wird, ob sich mittels dieser Substratgeometrie die Prozesse während der Entnetzung von Nickelschichten in fester Phase dahingehend beeinflussen lassen, dass die Agglomeration in länglichen Strukturen von Nanostäben oder Nanodrähten resultiert. Hierzu wurden zu den bereits in Abschnitt 6.2.1 behandelten Proben zusätzlich jeweils ein thermisch oxidiertes Siliziumsubstrat hochgradig geordneter Rippelstruktur der Wellenlänge $\lambda=42 \mathrm{~nm}$ (Abschnitt 4.2.2.3) simultan mitbedampft und in analoger Weise in situ bei entsprechenden Temperaturen ausgelagert. Anschließend wurden morphologische Untersuchungen mittels REM und AFM sowie Untersuchungen des Magnetisierungsverhaltens mittels MOKE durchgeführt. Die AFM-Messungen sind in Anhang E aufgeführt.

\subsubsection{Morphologieentwicklung}

Die bei Raumtemperatur deponierte Schicht (Abbildung 6.16(a)) weist in ihrem ursprünglichen Zustand wie auf glattem Substrat eine mit Furchen durchzogene, körnige Struktur auf, die keine erkennbaren Löcher besitzt, sodass der Film das Substrat anfangs vollständig bedeckt. Die Rippelstruktur ist bei reduzierter Rippeltiefe und Rauigkeit von $d_{R}=5 \mathrm{~nm}$ und $\sigma_{\mathrm{RMS}}=1.5 \mathrm{~nm}$ gegenüber der freien Substratoberfläche $\left(\mathrm{d}_{\mathrm{R}}=8 \mathrm{~nm}\right.$ und $\left.\sigma_{\mathrm{RMS}}=2.5 \mathrm{~nm}\right)$ auch auf der Nickeloberfläche deutlich ausgeprägt. Das Nickel setzt sich folglich nicht direkt bei der Deposition ausschließlich in die Täler, sondern adaptiert weitestgehend die zugrunde liegende Topographie. Die beobachtete Ausglättung der Oberfläche impliziert hierbei eine etwas geringere Schichtdicke auf den Bergrücken. Bei $100^{\circ} \mathrm{C}$ Auslagerungstemperatur (Abbildung 6.16(b)) ist ein Aufbrechen des Films zu beobachten und es bilden sich längliche Löcher, deren präferentielle Ausrichtung mit der Orientierung der Rippel übereinstimmt. Da die schmalen Löcher in den AFM-Messungen nicht zu identifizieren sind, kann nicht genau belegt werden, dass diese vornehmlich auf den Hügeln entstehen, auch wenn dies nahe liegt. Mit steigender Temperatur (Abbildung 6.16(c)) wird die Schicht durch Materialansammlung in den Tälern der Rippelstruktur in längliche Strukturen umgewandelt, welche zunächst noch über die Hügel hinaus verzweigt sind. Bei $300^{\circ} \mathrm{C}$ (Abbildung 6.16(d)) verschwinden diese Verzweigungen, die länglichen Strukturen nehmen entsprechend an Breite zu und es entwickelt sich ein Feld von isolierten Nanostäben mit breiter Längenverteilung und maximaler Länge von 400nm sowie einer mittleren Breite von etwa 20nm. Weitere Temperaturerhöhung (Abbildung 6.16(e)) führt zu breiteren Stäben mit reduzierter Länge, wobei auch nahezu periodisch angeordnete kompakte Inseln ähnlicher Größe auftreten, was auf eine Art Rayleigh-Instabilität hindeutet (siehe Abschnitt 6.3.3.2). 

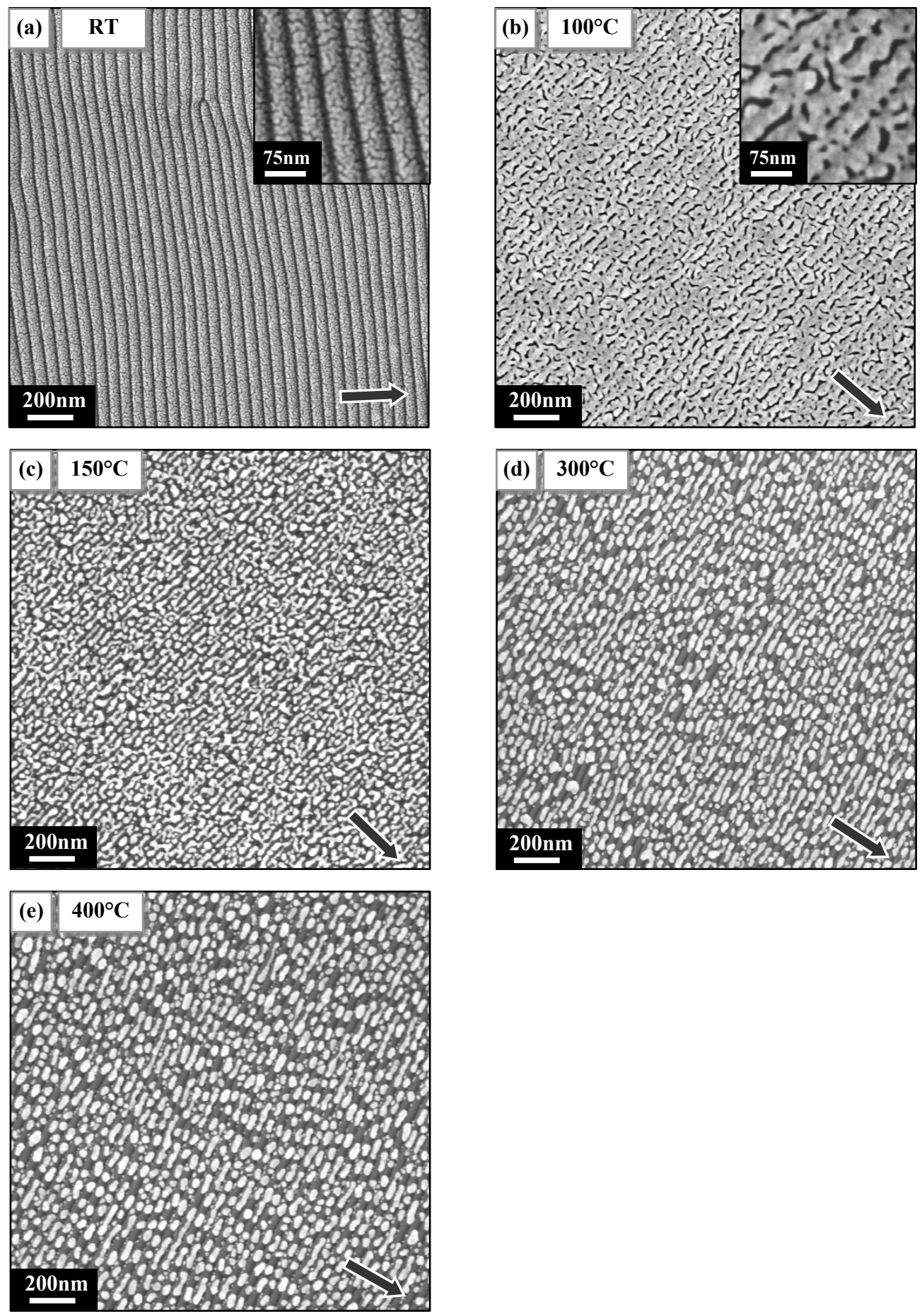

Abbildung 6.16: REM-Aufnahmen der bei unterschiedlichen Temperaturen ausgelagerten $5 \mathrm{~nm}$ Nickelschichten auf rippelstrukturiertem Substrat. Der Pfeil zeigt dabei in Richtung des Wellenvektors der Rippelstruktur. 

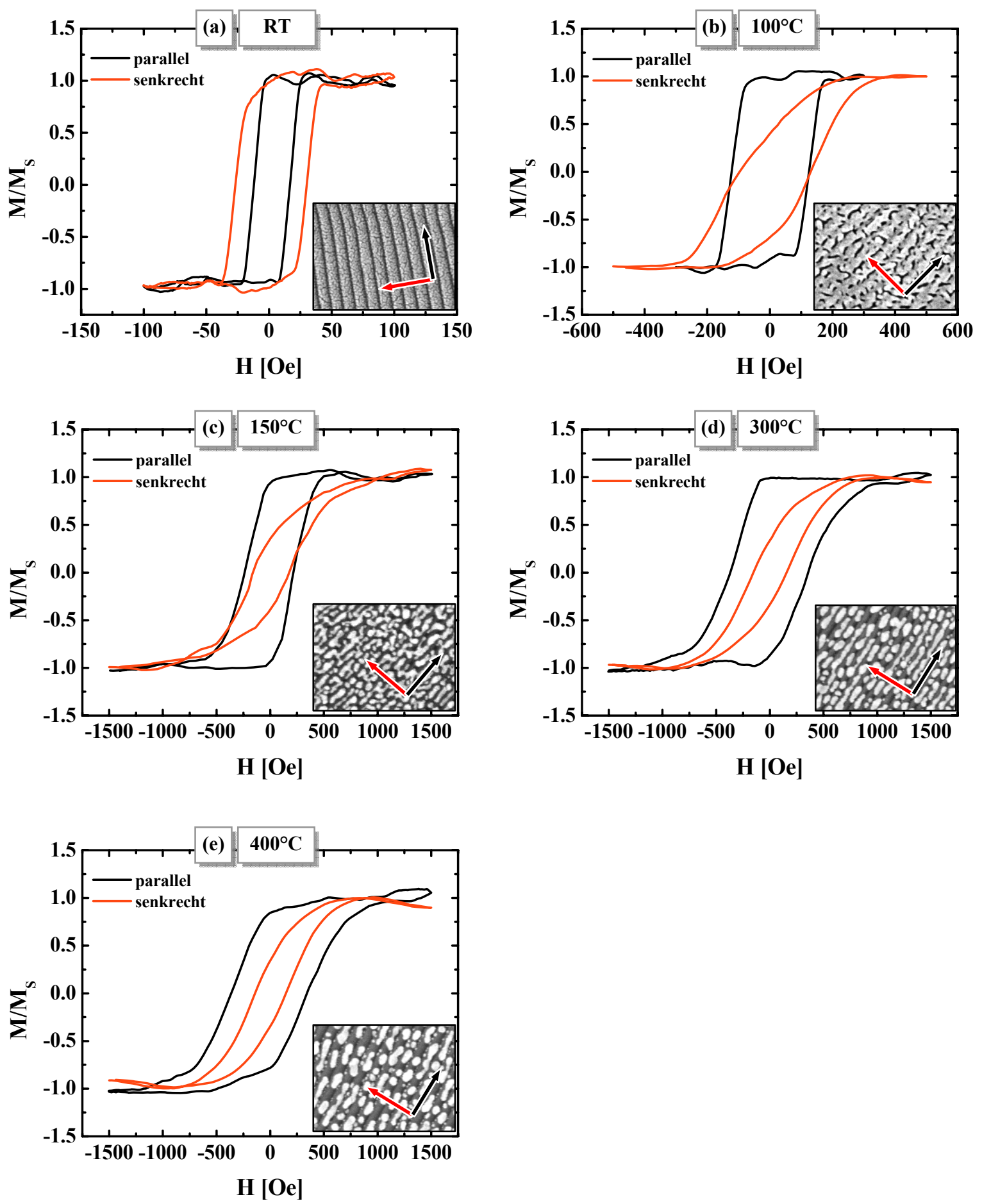

Abbildung 6.17: Magnetisierungsverhalten der bei unterschiedlichen Temperaturen ausgelagerten $5 \mathrm{~nm}$ Nickelschichten auf rippelstrukturiertem Substrat. 


\subsubsection{Magnetisierungsverhalten}

Im Gegensatz zu den bei erhöhter Temperatur deponierten Schichten des vorangegangenen Kapitels weisen die während der Entnetzung entstehenden Strukturen eine stabförmige Geometrie auf, die eine magnetische Formanisotropie nahe legt. Ferner ist neben dem Einfluss von zusätzlichen Defekten durch die Lochbildung auch eine magnetische Entkopplung der isolierten Strukturen zu vermuten. Um den entsprechenden Auswirkungen des Agglomerationsprozesses näher auf den Grund zu gehen, wurde das Magnetisierungsverhalten der Nickelschichten mittels magnetooptischen Kerreffekts bei paralleler als auch senkrechter Ausrichtung des äußeren Magnetfeldes bezüglich der zugrunde liegenden Rippelstruktur untersucht (Abbildung 6.17). Die unbehandelte Probe weist zunächst in beiden Richtungen ein ähnliches Magnetisierungsverhalten auf. Nach thermischer Behandlung offenbart sich eine klare Veränderung der Kurven mit signifikant geringerer Hysterese in senkrechter Ausrichtung.

Die Abhängigkeit der Remanenzmagnetisierung sowie des Koerzitivfeldes von der Auslagerungstemperatur sind in Abbildung 6.18 graphisch aufgetragen. Hierbei zeigt sich zum einen eine deutliche Abnahme der Remanenz bis $150^{\circ} \mathrm{C}$ in senkrechter Ausrichtung, welche genau bis zu der Temperatur auftritt, bei der sich isolierte Strukturen entwickeln, und auf die Ausbildung einer uniaxialen Anisotropie mit leichter Richtung parallel zu den Hügelketten der Oberflächenkorrugationen hindeutet. Die Remanenz in leichter Richtung sinkt erst bei $400^{\circ} \mathrm{C}$ etwas unter den Sättigungswert der Magnetisierung. Zum anderen wird beobachtet, dass der Nickelfilm bereits vor der thermischen Behandlung ein höheres Koerzitivfeld in senkrechter Richtung aufweist. Mit zunehmender Temperatur steigt das Koerzitivfeld in beiden Richtungen um grob eine Größenordnung, während es in senkrechter Richtung ab $150^{\circ} \mathrm{C}$ wieder leicht zurückgeht und in paralleler Richtung bei $300^{\circ} \mathrm{C}$ stagniert.
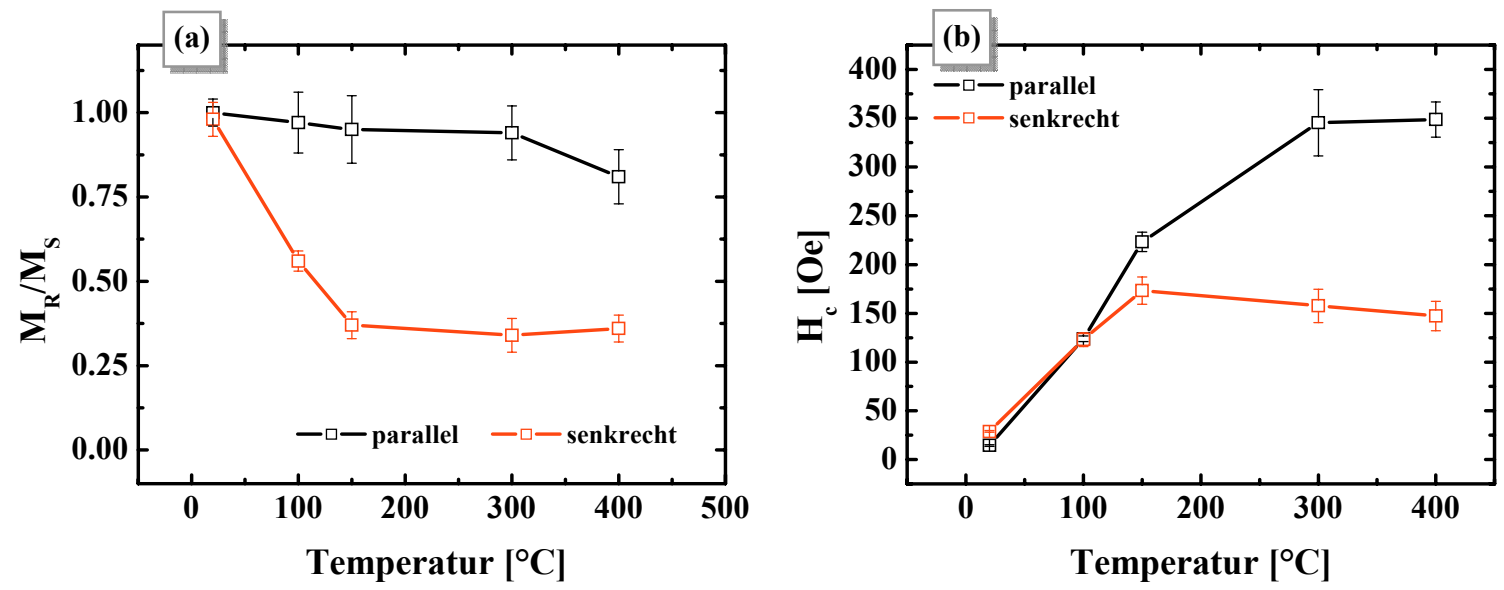

Abbildung 6.18: (a) Remanenzmagnetisierung und (b) Koerzitivfeld in Abhängigkeit von der Auslagerungstemperatur. 


\subsubsection{Diskussion}

\subsubsection{Entnetzungskinetik}

Die Untersuchungen der Strukturbildung durch Entnetzung zeigen einen klaren Unterschied in der Kinetik auf glattem und auf rippelstrukturiertem Substrat. Um dies zu verdeutlichen, ist in Abbildung 6.19 die prozentuale Oberflächenbedeckung der Nickelschicht aufgetragen sowie die entsprechenden REM-Aufnahmen einander gegenübergestellt. Ausgangssituation vor thermischer Behandlung ist in beiden Fällen ein nach Deposition vollständig bedeckender Film. Bei $100^{\circ} \mathrm{C}$ bilden sich nur wenige Löcher auf glattem Substrat, während der Film auf dem Rippelsubstrat bereits von langen Kluften durchzogen ist und die Oberflächenbedeckung der Nickelschicht rapider abfällt. Diese Tendenz setzt sich bis zu einer Temperatur von $300^{\circ} \mathrm{C}$ fort, bei der sich auf glattem Substrat zwar beachtliche Löcher geformt haben, deren Durchmesser ein Vielfaches der Rippelwellenlänge beträgt, jedoch nach wie vor eine zusammenhängende Schicht vorhanden ist, wohingegen sich auf der rippelstrukturierten Probe bereits isolierte Strukturen ausgebildet haben. Die Anisotropie der Oberflächentextur und -krümmung ruft dabei einen gerichteten Materialtransport hervor. Die Gradienten der Krümmung resultieren infolge der damit verbundenen Gradienten des chemischen Potentials in einer zusätzlichen treibenden Kraft in Form von krümmungsinduzierter Diffusion von den Bergkämmen in die Täler.

Bei höheren Temperaturen ist der Agglomerationsprozess auf glatter Oberfläche stärker ausgeprägt und es entstehen Inseln mit einem Durchmesser von bis zu 150nm. Demgegenüber wirken die Bergrücken der Rippel auf Grund der negativen Krümmung und des somit höheren chemischen Potentials wie Diffusionsbarrieren, folglich werden kaum Strukturen beobachtet, deren Breite die Wellenlänge der Rippel überschreitet, und das Nickel ist in den Senken gefangen. Dies bewirkt eine drastische Reduktion der Agglomerationsdynamik und die Oberflächenbedeckung stagniert nahezu. Jedoch besitzen die Nanostäbe oder Nanodrähte ein hohes Aspektverhältnis und damit auch ein großes Oberflächen-zu-VolumenVerhältnis. Ein vollständiges Zusammenziehen eines Stäbchens zu einer einzigen kompakten Insel ist auf Grund der lateralen Begrenzung durch die Diffusionsbarrieren nicht möglich. Die Anfälligkeit dünner zylindrischer Drähte gegenüber Durchmesserfluktuationen, bekannt als Rayleigh-Instabilität (siehe Abschnitt 6.3.3.2), sowie das Aufbrechen an den instabilen Korngrenzen der vermutlich polykristallinen Strukturen sorgt letztlich für den Zerfall der Nanodrähte.

Die spezielle Oberflächengeometrie der rippelstrukturierten Templates besitzt somit einen erheblichen Einfluss auf den strukturbildenden Entnetzungsvorgang. Die unterschiedliche Kinetik resultiert offenbar weitestgehend aus der zusätzlichen krümmungsinduzierten Oberflächendiffusion und den als Diffusionsbarrieren agierenden Hügelketten, wobei die unidirektionale Oberflächenmodulation eine entsprechend eindimensionale Ausdehnung der Partikel prägt. 


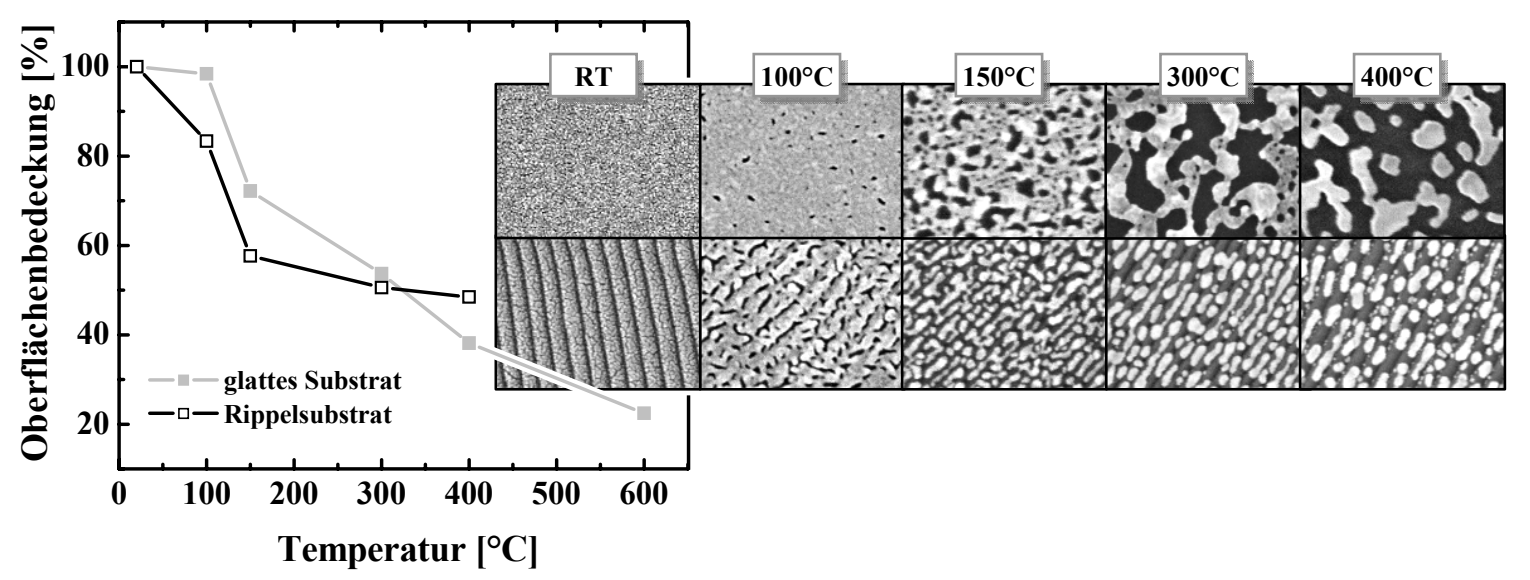

Abbildung 6.19: Oberflächenbedeckung des Nickelfilms während der Entnetzung auf glattem und auf rippelstrukturiertem Substrat sowie Gegenüberstellung der entsprechenden REM-Aufnahmen identischen Maßstabs.

\subsubsection{Rayleigh-Instabilität}

Lord Rayleigh [Ray78] analysierte die Instabilität eines dünnen, unendlich langen, zylindrischen Wasserstrahls, welche diesen unter dem Einfluss der Oberflächenspannung in eine Kette aus periodisch angeordneten Flüssigkeitstropfen zerfallen lässt. Nichols und Mullins [Nic65] haben die Theorie der Rayleigh-Instabilität auf Festkörper erweitert und zu diesem Zweck das viskose Fließen durch den Transportmechanismus der Diffusion substituiert. Ausgehend von einer infinitesimalen sinusförmigen Störung des Radius $r$ der Form $r=r_{0}+\alpha \cos (2 \pi x / \lambda)$ entlang des Zylinders, wobei $\alpha$ die Amplitude, $\lambda$ die Wellenlänge der Störung und $r_{0}$ den Radius des ungestörten Zylinders bezeichnet, wird erwartet, dass die Amplitude der Pertubationen mit einer Wellenlänge $\lambda>2 \pi \mathrm{r}_{0}$ exponentiell anwächst und diese Störungen somit destabilisierend wirken. Insbesondere besitzt die Störung mit der Wellenlänge $\lambda_{\mathrm{m}}=2 \pi \sqrt{2} \mathrm{r}_{0} \approx 8.89 \mathrm{r}_{0}$ bei vorherrschender Oberflächendiffusion die höchste Wachstumsrate, sodass Zylinder fester Phase schließlich in eine lineare Anordnung von Inseln mit Abstand $\lambda_{m}$ und Durchmesser $\mathrm{D}=3.78 \mathrm{r}_{0}$ umgewandelt werden. Weiteres Charakteristikum ist die $\mathrm{zu} \mathrm{r}_{0}^{2}$ proportionale Zeitkonstante des Zerfalls, weshalb sich die Instabilität letztlich auf sehr dünne Drähte beschränkt.

Diese Instabilität wurde in jüngster Zeit an meist einkristallinen Nanodrähten beobachtet [Mol04, Pen01]. Auch wenn hier keine belegbare Aussage über die Kristallinität der vermutlich polykristallinen Drähte gemacht werden kann, liegen die Abstände der bei höherer Temperatur entstandenen kompakten Partikel mit häufig auftretenden $75 \mathrm{~nm}$ im Bereich der Voraussagen der Rayleigh-Instabilität. Als Breite der Drähte kann ein mittlerer Durchmesser von etwa $20 \mathrm{~nm}$ abgeschätzt werden, sodass $\lambda_{\mathrm{m}} \approx 90 \mathrm{~nm}$ entspricht. Die Instabilität wird durch Polykristallinität nicht ausgeschlossen, jedoch führen die meist ebenfalls instabilen Korngrenzen zwangsläufig zu einer Störung der periodischen Anordnung resultierender 
Inseln, wodurch direkte Rückschlüsse auf das Auftreten einer Rayleigh-Instabilität erschwert werden. Des Weiteren besitzt die Anisotropie der Oberflächenenergie, welche in der Behandlung von Nichols und Mullins nicht berücksichtigt wurde, signifikanten Einfluss auf die resultierende Strukturbildung [Gla01]. Karim et al. [Kar07] haben beim Zerfall polykristalliner Goldnanodrähte durch Rayleigh-Instabilität einen um bis zu 30\% geringeren Partikelabstand gemessen als bei einkristallinen, was die hier vorliegenden Beobachtungen tendenziell bestätigt.

\subsubsection{Ausbildung von Nanodrähten}

Die Länge der Nanodrähte ist begrenzt. Dies wird schon im Frühstadium der Entnetzung deutlich. Die Unregelmäßigkeit in der Ausrichtung der länglichen Kluften bezüglich der Rippelorientierung in Abbildung 6.16(b) zeigt, dass die krümmungsinduzierte Diffusion, die ein Aufreißen der Schicht entlang der Bergkuppen und eine Materialansammlung in den Tälern hervorruft, nicht allein zur Morphologieentwicklung der Nickelschicht beiträgt. Sie wird überlagert von der gewöhnlichen kapillaritätsgetriebenen Entnetzung durch Furchenbildung an Korngrenzen, die das Agglomerationsverhalten der Schichten auf glatten Oberflächen bestimmt, wie in Abschnitt 6.2 demonstriert wurde, und zu einer unregelmäßigen Anordnung von Inseln führt. Wie in vielen Experimenten beobachtet, ist die Kinetik dieses Mechanismus schichtdickenabhängig. Jiran und Thompson [Jir90] fanden einen Zusammenhang $\mathrm{dx} / \mathrm{dt} \sim \mathrm{d}^{-3}$ zwischen der Wachstumsrate der Löcher $\mathrm{dx} / \mathrm{dt}$ und der Schichtdicke d.

Um möglichst lange zusammenhängende Nanostäbe zu erzeugen, erfordert die Wahl adäquater Parameter die kinetische Limitierung des gewöhnlichen Entnetzungsprozesses und die Dominanz krümmungsinduzierter Diffusion. Dünne Filme bilden schnell Löcher an Korngrenzen. Mullins [Mul57] hat gezeigt, dass die Furchenbildung an Korngrenzen für große Schichtdicken eingeschränkt ist. Eine größere Dicke des Films führt jedoch gleichzeitig zu einer stärkeren Ausglättung der Rippelstruktur während des Schichtwachstums und der Auffüllung der Täler, sodass die treibende Kraft für eine Entnetzung durch krümmungsinduzierte Diffusion reduziert wird. Folglich sinkt der Einfluss der TemplateGeometrie während des Entnetzungsvorgangs, wie die Morphologie einer thermisch behandelten 10nm Nickelschicht verdeutlicht (Anhang H). Für den Fall sehr dünner Filme hingegen sind die Körner unterhalb einer Schichtdicke von etwa 1.7nm (Abschnitt 6.2.2.1) nicht vollständig zusammengewachsen und, da Agglomerate mehrerer Atome weitestgehend immobil sind, wird die Anordnung der Inseln während der Deposition vorwiegend durch Oberflächendiffusion einzelner Adatome bestimmt. Auch die Deposition zusätzlichen Materials bei erhöhter Temperatur führt nicht zu einer ausgeprägten Koaleszenz präferentiell entlang der Rippel, wie die Untersuchungen aus Kapitel 5 gezeigt haben, sondern zur Ausbildung entlang der Täler aufgereihter kompakter Nanopartikel. In jedem Fall bewirken ausreichend hohe Temperaturen letztlich die Agglomeration des Films und Bildung 
isolierter Inseln entweder durch Furchenbildung an Korngrenzen selbst bei großen Schichtdicken oder durch die Rayleigh-Instabilität zunächst entstandener Nanodrähte. Folglich ist die Initiierung krümmungsinduzierter Diffusion bei kleinstmöglicher Temperatur beispielsweise durch Erhöhung der Krümmungsgradienten der zugrunde liegenden Oberflächentopographie des Templates anzustreben. Die Modifikation der Mikrostruktur bietet eine weitere Möglichkeit der Optimierung. Gemäß dem auf geometrischen Überlegungen beruhenden Modell von Srolovitz und Safran (Abschnitt 6.2.3.1) besitzen Filme geringer Korngröße bei gleicher Schichtdicke höhere Stabilität gegenüber thermischer Furchenbildung. Ein entsprechendes Gefüge ließe sich durch Deposition bei tiefen Temperaturen erzielen.

\subsubsection{Magnetisierungsverhalten}

Die Ummagnetisierung ferromagnetischen Materials durch ein äußeres Feld erfolgt im Allgemeinen durch Verschiebung von Domänenwänden, wobei Domänen, deren Magnetisierung etwa mit der Richtung des äußeren Feldes übereinstimmt, auf Kosten anderer wachsen. Nanopartikel besitzen eine kritische Größe, unterhalb der der Aufwand an Energie zur Bildung einer Domänenwand die zusätzliche Streufeldenergie eines Eindomänenzustands übersteigt. Für sphärische Nickelpartikel ohne magnetostatische Wechselwirkung der Teilchen untereinander liegt diese Grenze bei etwa 55nm. Haben die Partikel jedoch keine sphärische Gestalt sondern weisen eine Formanisotropie auf, erhöht sich der kritische Durchmesser [Lu07]. Alle Spins eines Eindomänenteilchens sind parallel ausgerichtet und zeigen in dieselbe Richtung, sodass eine Ummagnetisierung die simultane Rotation aller Spins erfordert, was für Partikeldurchmesser direkt unterhalb der kritischen Größe in einer erhöhten Koerzitivität resultiert. Wie bereits in Abschnitt 5.5.1 erläutert, führt das Unterschreiten des superparamagnetischen Limits mit abnehmender Dimension der Nanostrukturen indes zum Verschwinden von Remanenz und Koerzitivität.

Die Messungen des Magnetisierungsverhaltens der thermisch behandelten Nickelschichten lassen die Ausbildung einer Anisotropie erkennen, wobei in Verbindung mit den Beobachtungen der Morphologieentwicklung auf eine magnetische uniaxiale Formanisotropie zu schließen ist. Längliche Strukturen besitzen bedingt durch die hohe Streufeldentwicklung bei Magnetisierung in schwerer Richtung infolge der speziellen Geometrie eine hohe Anisotropieenergie. Konsequenz ist die beobachtete Reduktion der Remanenz in schwerer Richtung, wobei die Remanenz in leichter Richtung in etwa der Sättigungsmagnetisierung entspricht. Bis einschließlich $150^{\circ} \mathrm{C}$ Auslagerungstemperatur, bei der sich isolierte Strukturen ausbilden, die zunächst noch über die Rippel hinaus verzweigt sind, zeigt sich eine signifikante Änderung des Magnetisierungsverhaltens mit starkem Anstieg der Koerzitivität. Während sich Domänenwände in dem kontinuierlichen Film der unbehandelten Probe vermutlich weitestgehend ungehindert verschieben lassen, können die im Frühstadium der 
Entnetzung nukleierten Löcher sogenannte Pinning-Zentren darstellen. Die Bewegung der Domänenwände erfordert somit zusätzliche magnetische Energie, um den Wechselwirkungsbereich der Löcher wieder zu verlassen, was schließlich zu einer Verbreiterung der Hysterese und Erhöhung des Koerzitivfeldes führen kann. Bei der Auslagerungstemperatur von $400^{\circ} \mathrm{C}$ ist auch die Remanenz in leichter Richtung merklich reduziert, was auf die $\mathrm{Ab}-$ nahme der lateralen Ausdehnung in Längsrichtung der Nanodrähte schließen lässt und den Zerfall in kleinere, kompakte Partikel belegt. Die magnetischen Messungen zeigen somit kein unerwartetes Verhalten sondern bestätigen die morphologische Entwicklung.

\subsection{Einfluss von Silber}

Um den Einfluss einer nicht mischenden Komponente auf den Entnetzungsprozess der Nickelfilme zu untersuchen, wurden NiAg-Legierungsschichten von 10nm Dicke in einem Stöchiometrieverhältnis von 1:1 auf glattem sowie rippelstrukturiertem Substrat bei Raumtemperatur deponiert. Auf Grund der hohen Mischungsenthalpie und marginaler Randlöslichkeit [Mas86] ist hierbei von einer zweiphasigen Legierung auszugehen. Die Filme wurden mittels Sputterdeposition hergestellt, wobei durch die Verwendung eines Abschirmbleches im Abstand von $\mathrm{s}<1 \mathrm{~mm}$ zum Substrat ein Teil des Substrats abgeschattet wurde und somit ein Bereich lokal variierender Schichtdicke erzeugt wurde (Abbildung 6.20). Anschließend wurden die Proben bei einer Temperatur von $300^{\circ} \mathrm{C}$ in situ für eine Stunde

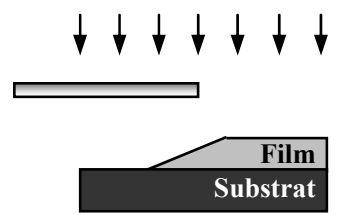
Abbildung 6.20: Deposition mit variierender Schichtdicke. ausgelagert und bei Raumtemperatur mittels REM charakterisiert.

\subsubsection{Entnetzung von NiAg auf Rippelstrukturen}

Abbildung 6.21 zeigt REM-Aufnahmen des thermisch behandelten NiAg-Films auf rippelstrukturiertem Substrat an Orten unterschiedlicher Schichtdicke. Bei geringer Dicke (Abbildung 6.21(a)) ist die Schicht zersetzt in überwiegend kreisrunde isolierte Inseln, welche mehrheitlich in Ketten entlang der Rippel ausgerichtet sind und auf einzelnen Abschnitten eine periodische Anordnung aufweisen. Mit zunehmender Schichtdicke wächst die laterale Ausdehnung entlang der Täler und somit das Aspektverhältnis der Strukturen (Abbildung 6.21(b)-(c)) bis schließlich im mittleren Schichtdickenbereich Nanodrähte mit einer Länge von bis zu $2 \mu \mathrm{m}$ beobachtet werden (Abbildung 6.21(d)). $\mathrm{Zu}$ größeren Schichtdicken hin nimmt die Verzweigung der Drähte senkrecht zu den Rippeln über die Hügelkuppen hinaus zu (Abbildung 6.21(e)) bis die Schicht ganze Bereiche aufweist, die sich über das Vielfache der Rippelwellenlänge erstrecken und das Substrat vollständig bedecken, sodass vielmehr von einem zusammenhängenden Film gesprochen werden muss, der lokal Bereiche drahtförmiger verzweigter Löcher aufweist (Abbildung 6.21(f)). Diese Mor- 


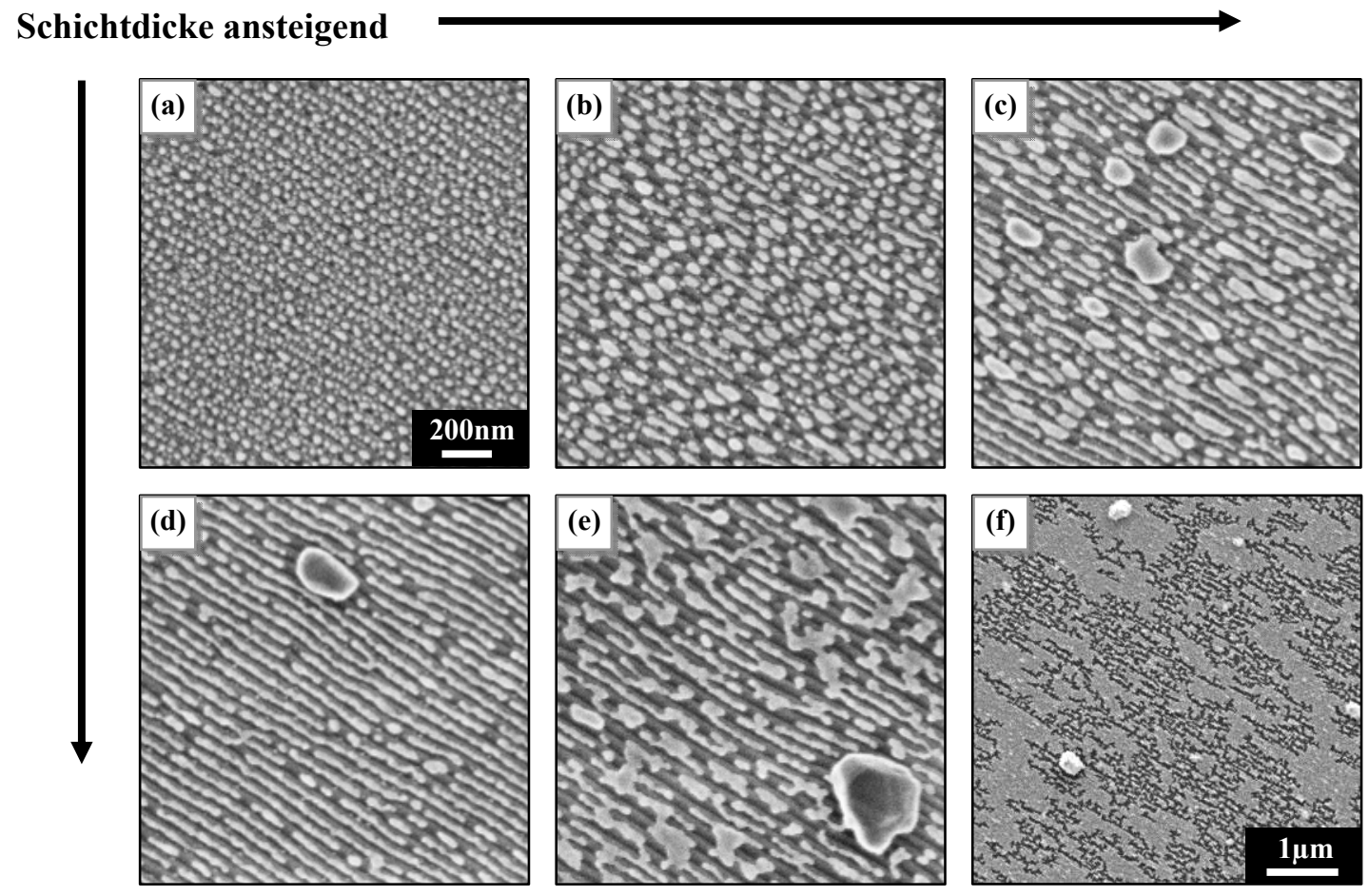

Abbildung 6.21: REM-Aufnahmen der bei $300^{\circ} \mathrm{C}$ ausgelagerten NiAg-Schicht auf rippelstrukturiertem Substrat. Die Schichtdicke ist von (a) nach (f) zunehmend und beträgt maximal 10nm. Abgesehen von (f) ist der Maßstab aller Aufnahmen identisch.

phologie wird auch auf dem nicht abgeschatteten Teil der Probe beobachtet, auf dem die maximale Schichtdicke von $10 \mathrm{~nm}$ vorliegt.

Neben den Nanodrähten bilden sich überdies ab einer gewissen Schichtdicke vereinzelt größere kompakte Partikel (Abbildung 6.21(c)-(f)), deren Abstand und Größe mit der Schichtdicke anwachsen, wodurch sie sich immer stärker von der übrigen Mikrostruktur absetzen. Bei maximaler Schichtdicke von 10nm weisen diese Partikel einen Abstand von mehreren Mikrometern und einen Durchmesser von etwa 400nm auf.

\subsubsection{Entnetzung von NiAg auf glattem Substrat}

Die REM-Aufnahmen des entsprechenden NiAg-Films auf glattem Substrat sind in Abbildung 6.22 sowie mit deutlich geringerer Vergrößerung in Abbildung 6.23 dargestellt. Die heller erscheinenden Ränder in Abbildung 6.22(d)-(f) sind das Resultat von Aufladungseffekten infolge der nichtleitenden Substratoberfläche.

Bei kleiner Schichtdicke (Abbildung 6.21(a)) entwickeln sich zunächst wie auf rippelstrukturiertem Substrat überwiegend kreisrunde Inseln, die ohne Einfluss der speziellen Substrattopographie regellos angeordnet sind. Mit zunehmender Dicke entsteht auch hier 


\section{Schichtdicke ansteigend}
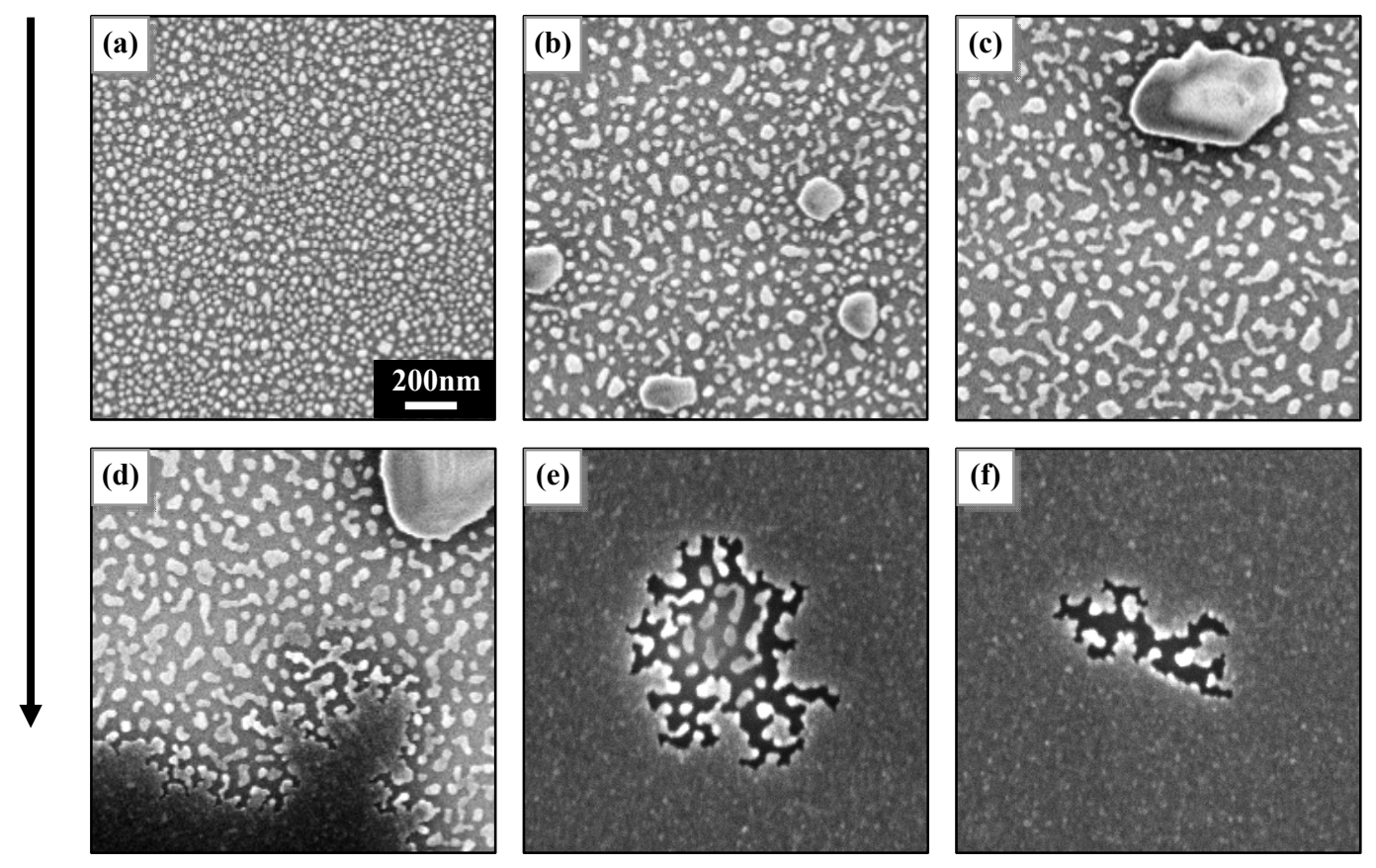

Abbildung 6.22: REM-Aufnahmen der bei $300^{\circ} \mathrm{C}$ ausgelagerten NiAg-Schicht auf glattem Substrat. Die Schichtdicke ist von (a) nach (f) zunehmend und beträgt maximal 10nm. Der Maßstab aller Aufnahmen ist identisch.

ein Gefüge aus kleinen Inseln und größeren kompakten Partikeln, deren Größe und Abstand mit der Schichtdicke anwachsen (Abbildung 6.21(b)-(c)). Schließlich weist die Probe auch hier einen zusammenhängenden Film auf, der von vereinzelten entnetzten Bereichen durchsetzt ist (Abbildung 6.21(d)-(f)), welche im nicht abgeschatteten Teil der Probe von 10nm Schichtdicke allerdings nicht auftreten.

Im Vergleich zu den Beobachtungen an reinen Nickelschichten offenbart sich jedoch ein anderes Entnetzungsverhalten. Während die Ergebnisse aus Abschnitt 6.2 zeigen, dass reine Nickelschichten eine Vielzahl von Löchern ausbilden, die nach dem gewöhnlichen kapillaritätsgetriebenen Entnetzungsmechanismus durch Wachstum und Koaleszenz isolierte Inseln erzeugen, bilden sich hier vergleichsweise wenige nahezu kreisförmige Bereiche inselartiger Strukturen, die offenbar jeweils aus einem einzigen Loch entstanden sind, welches sich zweigartig ausbreitet. Aus den Übersichtsbildern in Abbildung 6.23 wird deutlich, dass Dichte und Radius der kreisförmigen entnetzten Flächen mit abnehmender Schichtdicke ansteigen und es einen scharfen Übergangsbereich von wenigen Mikrometern Breite zwischen isolierten und überlappenden Flächen gibt. Über die Schichtdickenvariation innerhalb dieses Bereiches kann keine verlässliche Aussage getroffen werden, da die geometrische Abschattung von einer Reihe von Faktoren wie beispielsweise dem Gasdruck und der Spannung während des Sputterprozesses sowie dem genauen Abstand des Targets und des Abschirmbleches zur Substratoberfläche abhängig ist, deren Einfluss speziell auf Grund des 

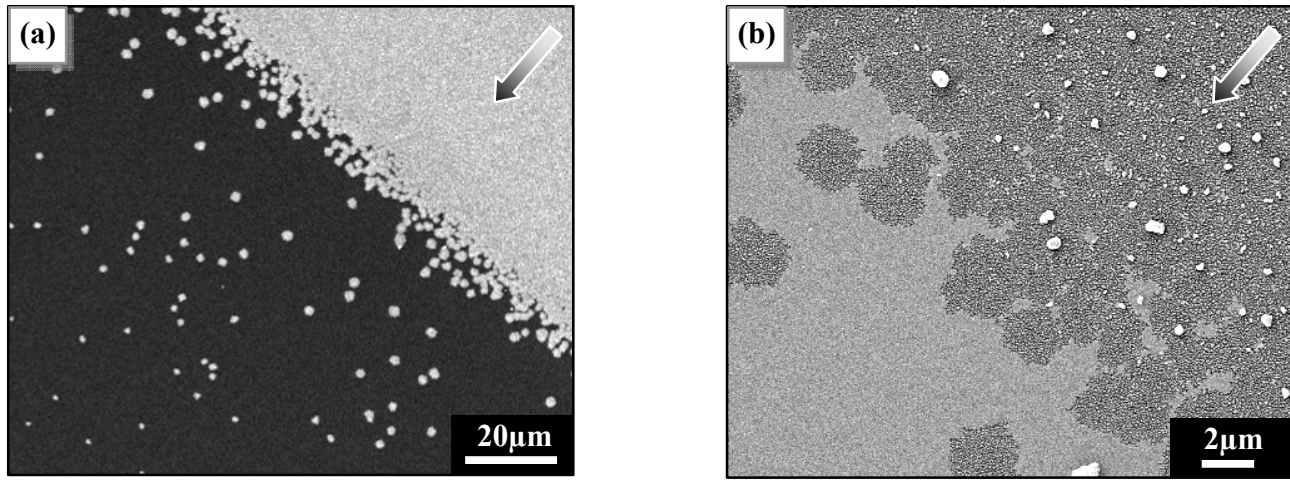

Abbildung 6.23: REM-Aufnahmen der bei $300^{\circ} \mathrm{C}$ ausgelagerten $\mathrm{NiAg}-\mathrm{Schicht}$ auf glattem Substrat. Die Schichtdicke ist in Pfeilrichtung zunehmend. In (b) ist der Einfluss lokaler Aufladungseffekte durch Verwendung des im REM außerhalb der Elektronenoptik angebrachten Sekundärelektronendetektors reduziert, sodass die entnetzten Bereiche hier dunkel erscheinen.

sehr geringen und nicht genau bekannten Abstands der Abschirmung zur Probe schlecht abzuschätzen ist. Während bei geringer Schichtdicke der Film offensichtlich infolge der Überlappung entnetzter Bereiche vollständig in isolierte Inseln agglomeriert ist, weist der nicht abgeschattete Teil der Probe mit einer Schichtdicke von 10nm einen geschlossenen Film völlig ohne Löcher auf, wohingegen auf der Rippelstruktur der Entnetzungsprozess selbst bei maximaler Schichtdicke bereits eingesetzt hat, was analog zu reinen Nickelschichten die höhere thermische Stabilität ohne zugrunde liegende Rippelstruktur belegt.

\subsubsection{Diskussion}

\subsubsection{Morphologieentwicklung auf Rippelstrukturen}

Wie bereits zuvor diskutiert, wird die Anordnung von Inseln sehr dünner und nicht vollständig koaleszierter Schichten bereits während der Deposition durch Diffusion von Adatomen auf der Substratoberfläche bestimmt. Bei Raumtemperatur ist die mittlere Diffusionslänge klein gegenüber der Rippelwellenlänge und führt folglich zu einer regellosen Anordnung der Inseln unabhängig von der Rippelstruktur. Die linear organisierten Inseln ähnlich in Größe und Abstand in Abbildung 6.21(a) sind ein Anzeichen für Zersetzung durch Rayleigh-Instabilität und indizieren, dass sich zunächst Nanodrähte gebildet hatten, welche auf Grund geringen Durchmessers bei $300^{\circ} \mathrm{C}$ nicht stabil waren. Mit der Schichtdicke steigt auch die thermische Stabilität gegenüber Furchenbildung [Mul57] und das zusätzliche Material führt zur Bildung langer Nanodrähte, die sich auf Grund ihrer größeren Breite auch durch erhöhte Stabilität bezüglich Durchmesserfluktuationen auszeichnen. Das bei großen Schichtdicken auftretende Verhalten nur lokaler Zersetzung (Abbildung 6.21(f)) wider- 
spricht dem in Abschnitt 6.2 beobachteten Entnetzungsmechanismus von Nickel und wird im folgenden Abschnitt diskutiert.

Zur Klärung der Frage nach der Distribution des Silbers werden die Ergebnisse des vorangegangenen Kapitels herangezogen, von denen speziell die Untersuchungen an den unterschiedlichen thermisch behandelten NiAg-Legierungsschichten sowie Ni/Ag-Doppellagen von Interesse sind. Hierbei hat sich gezeigt, dass Silber in Verbindung mit Nickel an die Oberfläche segregiert und große Partikel in einem Abstand von mehreren Mikrometern formt, ähnlich derer, die in Abbildung 6.21(c)-(f) zu beobachten sind. Die Ausbildung dieser Partikel bedingt jedoch einen zunächst geschlossenen Metallfilm, weshalb zu kleinen Schichtdicken hin infolge der geringen thermischen Stabilität und des schnellen Zersetzens dünner Filme die Diffusion des Silbers frühzeitig eingeschränkt wird und es auf den isolierten Nickelinseln gefangen ist. Daher bildet sich bei kleiner Schichtdicke (Abbildung 6.21(a)-(b)) keine signifikant zweiphasige Morphologie aus. Bei großer Schichtdicke löst sich das Silber hingegen von der zurückbleibenden Nickelschicht und die deutlich hervortretenden kompakten Partikel (Abbildung 6.21(c)-(f)) können als Silberpartikel identifiziert werden.

\subsubsection{Entnetzungsmechanismus}

Die Agglomeration eines polykristallinen geschlossenen Films wird als das Resultat kapillaritätsgetriebener Furchenbildung an Korngrenzen angesehen, wie es beispielsweise für die Metalle Kupfer [Kwo03], Silber [Sie01], Palladium [Eri99] und Ruthenium [Gad05] berichtet wurde. Auch für Goldschichten auf $\mathrm{SiO}_{2}$ wurde dies zunächst angenommen [Jir90, Hum81].

Kürzlich haben Kwon et al. [Kwo03] in einer Vergleichsstudie entnetzender Gold- und Kupferschichten auf $\mathrm{SiO}_{2}$ ein vom üblichen Entnetzungsverhalten abweichendes, fraktalähnliches Wachstum der Löcher für Gold beobachtet (Abbildung 6.24), ähnlich den Ergebnissen der hier untersuchten NiAg-Schichten. Sie fanden, dass im Gegensatz zum gewöhnlichen Entnetzungsmechanismus die Anzahldichte nukleierter Löcher beträchtlich geringer ist und nach einer gewissen Nukleationsphase stagniert. Die begrenzte Anzahl von Nukleationspunkten des fraktalen $\mathrm{Ag}$ glomerationsmechanismus wurde darauf zurückgeführt, dass diese vermutlich durch lokale Anhäufungen von Überschussleerstellen der gesputterten Filme gebildet werden. Dabei vermuteten sie, dass mechanische Spannungen die Ursache für dieses Phänomen sind,
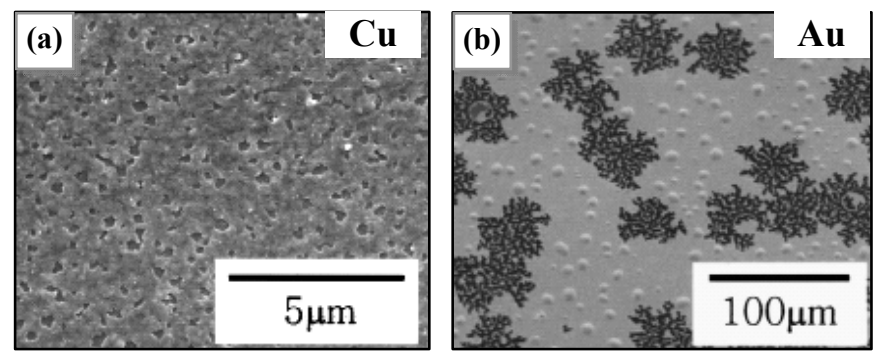

Abbildung 6.24: REM-Aufnahmen 50nm dicker entnetzender (a) Kupfer- und (b) Goldschichten [Kwo03]. 
konnten jedoch nicht schlüssig klären, was Gold von anderen Materialien unterscheidet und letztlich diesen speziellen Entnetzungsmechanismus induziert.

Gadkari et al. [Gad05] fanden ähnliche Ergebnisse für sowohl Gold- als auch Platinschichten und schrieben dies der geringen Adhäsion zum Substrat zu. Sie beobachteten, dass Blasenbildung eine stets auftretende Begleiterscheinung der fraktalen Entnetzungsmode ist und deuteten diese als partielle Filmablösung, hervorgerufen durch Druckspannungen des sputterdeponierten Films. Im Gegensatz zu Kwon et al., welche die Blasen (Abbildung 6.24(b)) als Argongaseinschlüsse interpretierten und ihnen keinen Einfluss auf den Entnetzungsprozess zugeschrieben haben, betrachteten Gadkari et al. diese quasi freistehenden Bereiche der Schicht als präferentielle Gebiete der Nukleation von Löchern sowie als Ursache für eine lineare Ausbreitung, welche auf Grund der Reduktion des erforderlichen Materialtransports im Vergleich zu einem sich kreisförmig ausbreitenden Loch zu einem fraktalen Wachstum führt. Sie vermuten daher eine morphologische Instabilität, die simultan an beiden Oberflächen einer freistehenden Schicht auftritt im Gegensatz zur Oberflächeninstabilität eines Films mit starker Haftung zum Substrat.

Die hier gewonnenen Ergebnisse zeigen, dass durch Zugabe von Silber eine Änderung des Entnetzungsmechanismus von kapillaritätsgetriebener Nukleation und Wachstum sich ausbreitender Löcher durch Furchenbildung an Korngrenzen und Oberflächendiffusion, wie es an reinen Nickelschichten beobachtet wurde, zur fraktalen Wachstumsmode der Löcher induziert wird, wie sie von Kwon et al. und Gadkari et al. für Gold- und Platinschichten berichtet wurde. Es wurden jedoch weder ein Auftreten von Delamination in Form von Blasenbildung noch Anzeichen für geringere Adhäsion der NiAg-Schichten zum Substrat gefunden. Da Untersuchungen an sputterdeponierten reinen Nickelfilmen (Anhang I) denselben Entnetzungsmechanismus wie aufgedampfte Nickelfilme offenbarten, ist ein reiner Effekt des Sputterdepositionsverfahrens, welches häufig zu Druckspannungen aber auch zu einer stärkeren Bindung zwischen Schicht und Substrat führt [Tho00], auszuschließen. Vielmehr lässt sich im hier untersuchten System über den Einfluss mechanischer Spannungen spekulieren.

Die Oberflächensegregation durch Herausdiffundieren des Silbers aus der Schicht legt die Erzeugung lokaler Zugspannungen sowie Relaxation durch Akkumulation von Poren und Leerstellen nahe, die zur Ausbildung einzelner Nukleationspunkte führen können. Die Zugspannungen destabilisieren Korngrenzen [Sro95] und die Löcher breiten sich vermutlich entlang schwach gebundener hochenergetischer Korngrenzen aus, wobei sie den Film förmlich zerreißen, sodass im Zuge der Spannungsrelaxation längliche, sich verzweigende Löcher entstehen.

Obwohl die durchgeführten Experimente den Ursprung der auftretenden fraktalen Wachstumsmode nicht im Detail klären können, indizieren sie basierend auf den oben genannten Annahmen die Relevanz mechanischer Spannungen. Der Übergang zwischen den unterschiedlichen Entnetzungsmechanismen von Nickel ist direkt verbunden mit der Ein- 
bringung der nicht mischbaren Komponente Silber, welches als eine Art Katalysator fungiert und ohne chemische Reaktion nach dem Entnetzungsvorgang in elementarer, vom Nickel separierter Form vorliegt. Auf rippelstrukturiertem Substrat unterstützen die auf Grund der Oberflächensegregation vermutlich auftretenden Zugspannungen durch das Zusammenwirken der Schichtausdünnung auf den Bergrücken infolge von krümmungsinduzierter Diffusion die Ausbildung von Nanodrähten. Weitere Systematische Untersuchungen speziell hinsichtlich des Einflusses mechanischer Spannungen sind erforderlich, um die unterschiedliche Natur der beiden Entnetzungsmechanismen zu klären. 


\section{Kapitel}

\section{Zusammenfassung und Ausblick}

In der vorliegenden Arbeit wurde die Entwicklung der Grenzflächenrauigkeit der inneren Phasengrenze im nicht mischbaren System Ni-Ag unter Ionenbestrahlung im Hinblick auf die Identifikation dominierender kinetischer Prozesse untersucht. Weiterhin wurden Untersuchungen zur Erzeugung isolierter metallischer bzw. magnetischer Nanostrukturen durch die gezielte Steuerung von unterschiedlichen Selbstorganisationsprozessen in dünnen Niund NiAg-Filmen durchgeführt. Hierzu wurde der Einfluss der Oberflächenmorphologie des Substrats am Beispiel rippelstrukturierter Templates analysiert. Bei den Selbstorganisationsprozessen handelte es sich zum einen um die hinlänglich bekannten Prozesse des Schichtwachstums während der Deposition. Zum anderen wurde die Agglomeration zunächst geschlossener Filme durch thermisch aktivierte Entnetzung in fester Phase studiert.

\section{Glättung innerer Ni-Ag-Grenzflächen}

Mittels Röntgenreflektivitätsmessungen an Ni/Ag-Doppellagen konnte gezeigt werden, dass die innere Phasengrenze des Systems unter Ionenbestrahlung im Energiebereich überwiegend nuklearen Energieverlustes $\left(300 \mathrm{keV} \mathrm{Xe}^{+}\right)$ähnlich der morphologischen Entwicklung von Oberflächen konkurrierenden Glättungs- und Aufrauungsmechanismen unterworfen ist. Die bei geringen Fluenzen beobachtete Abnahme der Rauigkeit wird dominiert von Glättungseffekten getrieben durch Minimierung des Aufwands an Grenzflächenenergie. In Kombination mit den von S. G. Mayr begleitend durchgeführten MD-Simulationen, die den rapiden Rauigkeitsabfall bestätigten, konnte der ursächliche Materialtransport zur Grenzflächenglättung auf die Dominanz viskosen Fließens innerhalb der Thermal-Spike-Phase zurückgeführt werden. Bei hohen Fluenzen wurde ein nahezu linearer Anstieg der Rauigkeit beobachtet, der auf langreichweitigen ballistischen Transport während der Kollisionsphase hindeutet und eine zunehmende Aufweichung der Grenzfläche verursacht. Die Kinetik des simultan auftretenden Kornwachstums wird indes durch Erreichen von Korngrößen in der Dimension der Schichtdicke sowie strahlungsinduzierte Defekte eingeschränkt. Die Ergebnisse demonstrieren, dass es bei moderaten Fluenzen prinzipiell möglich ist, auch innere Grenzflächen lokal oder flächendeckend zu glätten, ohne entsprechende Proben bei- 
spielsweise einer für viele elektronische Bauteile schädlichen Wärmebehandlung zu unterziehen.

\section{Rippelbildung}

Zur Herstellung von Templates für die gezielte Beeinflussung der Selbstorganisationsprozesse wurden polierte Siliziumsubstrate unter schrägem Winkel mit Ionen bestrahlt. Hierdurch lassen sich bei geeigneter Wahl der äußeren Parameter wellenförmige Rippelstrukturen auf der Oberfläche erzeugen. Da bislang beobachtete Strukturen meist ein nur geringes Aspektverhältnis von Amplitude zu Wellenlänge sowie mangelnde Ordnung aufwiesen, wurden Untersuchungen speziell dahingehend geführt, bei kleiner Wellenlänge eine hohe Rippeltiefe und insbesondere einen hohen Ordnungsgrad und geringe Defektdichte zu erzielen. Die auf diesem Weg beobachteten Oberflächenmorphologien sowie Parameterabhängigkeiten wurden im Rahmen bekannter Modelle und experimenteller Daten aus der Literatur diskutiert. Unter Argonbestrahlung mit $\theta=60^{\circ}, \mathrm{E}=3.8 \mathrm{keV}$ und hoher Ionenstromdichte war es möglich, nahezu sinusförmige und hochgradig geordnete Rippel einer Wellenlänge von $\lambda=42 \mathrm{~nm}$ und im Rahmen geometrischer Restriktionen maximalen Rippeltiefe von $d_{R}=8 \mathrm{~nm}$ bei einer Defektdichte von etwa $1.5 \mu \mathrm{m}^{-2}$ zu präparieren. Die maßgebliche Einschränkung der transversalen Ausdehnung hochgradig geordneter Rippel konnte als eine sich in dreieckigen Strukturen mit einem Scheitelwinkel von 33(2) ${ }^{\circ}$ äußernde Störung identifiziert werden, welche direkt mit der Entstehung von Versetzungsrippeldefekten korreliert ist. Die Unabhängigkeit des Scheitelwinkels vom Ioneneinfallswinkel $\theta$ schließt dabei eine rein geometrische Ursache aus und suggeriert die Relevanz zusätzlicher Mechanismen. Zwecks des Einsatzes als Templates wurden die Substrate thermisch oxidiert, um eine chemisch inerte Oberfläche zu erzeugen.

\section{Selbstorganisation während der Deposition}

Die rippelstrukturierten Substrate wurden eingesetzt, um die Mechanismen des Schichtwachstums kodeponierter NiAg-Legierungsschichten hinsichtlich einer möglichen geometrischen sowie kompositionellen Ordnung gezielt zu beeinflussen. Dabei wurde zur Steigerung der Kinetik von Oberflächenprozessen sowie der Phasenseparation während der Deposition das Substrat auf erhöhter Temperatur von $600^{\circ} \mathrm{C}$ gehalten. Die elementspezifische Analyse mittels XPS, EDX und TEM ergab eine klare Oberflächensegregation des Silbers, die sich infolge der gegenüber Nickel geringeren Oberflächenspannung auf die Minimierung der Oberflächenenergie des Systems zurückführen lässt. REM-Untersuchungen sowie eine statistische Analyse der Inselgrößenverteilung haben gezeigt, dass die entstehenden separierten Nanopartikel bei geeigneter deponierter Materialmenge gegenüber der Deposition auf glattem Substrat eine deutlich schärfere Größenverteilung bei reduzierter mittlerer Größe ( $\overline{\mathrm{a}} \approx \lambda=42 \mathrm{~nm}$ ) aufweisen und in den Tälern der Rippelstruktur zu linearen Ketten angeordnet sind. Ferner offenbart die Fourier-Analyse eine hexagonale Nahordnung mit 
geringer Asymmetrie. Ein Einfluss auf die Kristallorientierung konnte indes nicht nachgewiesen werden. Für die Anordnung der Inseln ist hierbei nicht allein das Frühstadium des Wachstums entscheidend, dass durch zusätzliche krümmungsinduzierte Oberflächendiffusion der Adatome beeinflusst wird und somit die üblicherweise regellose Anordnung von Kristallisationskeimen unterminiert. Die Bergrücken wirken auf Grund negativer (konvexer) Krümmung und des folglich höheren chemischen Potentials während der Koaleszenz als Diffusionsbarrieren und verhindern ein Überwachsen, wodurch die laterale Ausdehnung der Inseln entscheidend eingeschränkt wird. Bei großer Schichtdicke ist infolge der Koaleszenz der Inseln über die Wellenberge hinaus indes kein Einfluss der zugrunde liegenden Substratmorphologie erkennbar. Die Verwendung rippelstrukturierter Substrate während der Schichtdeposition ermöglicht somit die Selektierung einer definierten Inselgröße über die Einstellung der Rippelwellenlänge bei geeigneter Schichtdicke und stellt ein prinzipiell einfaches Verfahren zur gezielten Herstellung linearer Ketten isolierter metallischer bzw. magnetischer Nanopartikel dar, deren Dimension und Verteilungsbreite sich mittels Parameteroptimierung vermutlich stark reduzieren lässt.

\section{Selbstorganisation durch Entnetzung}

Mittels REM- und AFM-Analyse sowie in situ Widerstandsmessung wurde die morphologische Entwicklung und Kinetik des Agglomerationsvorgangs geschlossener dünner NiFilme auf glattem $\mathrm{SiO}_{2}$-Substrat untersucht und die wesentlichen Mechanismen der Entnetzung durch thermische Behandlung in fester Phase analysiert sowie die unterschiedlichen Stadien des Entnetzungsprozesses charakterisiert. Kapillaritätsgetriebene Nukleation von Löchern durch Furchenbildung an Korngrenzen und nahezu kreisförmiges Wachstum bestimmen das Entnetzungsverhalten reiner Ni-Schichten. Lochbildung wird bei einer Schichtdicke von $5 \mathrm{~nm}$ bereits unterhalb von $100^{\circ} \mathrm{C}$ initiiert. Der elektrische Widerstand zeigt während der Agglomeration eine irreversible Abnahme von bis zu 70\%, was die Ausbildung stark defektreduzierter elektronischer Pfade indiziert. Der Film weist dabei halsähnliche Strukturen auf, deren Durchmesser die ursprüngliche Schichtdicke deutlich übersteigen, bevor Verengungen diese Kanäle unterbrechen und zum elektrischen Versagen führen. Auf Grund des geringeren Grenzflächen-zu-Volumen-Verhältnisses der Hälse und der Verminderung elektronischer Grenzflächenstreuung wird der Widerstand trotz der infolge isolierender Löcher erhöhten Anzahl an Störstellen erheblich reduziert. Über die Heizratenabhängigkeit konnte die Aktivierungsenergie des kinetisch limitierenden Prozesses der Agglomeration zu $0.32 \mathrm{eV}$ bestimmt und der vorherrschende atomare Transportmechanismus als Oberflächenselbstdiffusion auf den (111)-Ebenen der Nickeloberfläche identifiziert werden. Zudem war über das Wachstumsverhalten einer nachträglich deponierten Silberschicht ein Nachweis eines auf der Substratoberfläche zurückbleibenden dünnen Wetting-Layers möglich. 
Auf rippelstrukturierten Substraten wirken grundsätzlich dieselben Mechanismen, jedoch offenbart die Entnetzung hier eine deutlich veränderte Kinetik, die sich auf eine zusätzliche treibende Kraft durch krümmungsinduzierte Diffusion zurückführen lässt, welche einen gerichteten Materialtransport von den Hügeln in die Täler hervorruft. Bei identischer Temperatur werden deutlich größere, vornehmlich längliche Löcher beobachtet, die parallel zu den Rippeln orientiert sind. Die Ausbildung von Nanostäben bzw. Nanodrähten in den Tälern im weiteren Verlauf der Wärmebehandlung demonstriert dabei die Rolle der Bergrücken als Diffusionsbarrieren für die laterale Ausdehnung resultierender Strukturen, die vorwiegend auf Grund der Rayleigh-Instabilität einem weiteren Zerfallsprozess unterworfen sind. Eine Längenmaximierung der Drähte erfordert die kinetische Einschränkung des zu ungeordneten Strukturen führenden Mechanismus der Furchenbildung an Korngrenzen, der der krümmungsinduzierten Diffusion überlagert ist. Magnetisierungsmessungen bestätigen die morphologische Entwicklung entnetzender Schichten und zeigen die schrittweise Ausbildung einer uniaxialen Anisotropie bereits während der Nukleationsphase.

Durch Zugabe einer nicht mischenden Komponente (Silber) wurde eine Änderung des Entnetzungsmechanismus von kapillaritätsgetriebener Nukleation und Wachstum der Löcher zu einer fraktalen Wachstumsmode beobachtet, wobei nur wenige Löcher entstehen, die sich zweigartig ausbreiten. Das Silber segregiert an die Oberfläche und fungiert dabei als eine Art Katalysator für diesen Entnetzungsmechanismus. Der Segregationsprozess legt das Auftreten lokaler Zugspannungen nahe, die den Film entlang hochenergetischer Korngrenzen destabilisieren und über eine rissartige Ausbreitung der Löcher relaxieren. Auf Rippelstrukturen unterstützt dieser Prozess die Ausbildung geordneter Nanostrukturen und führt zu einer deutlichen Erhöhung der Drahtlänge von bis zu $2 \mu \mathrm{m}$. Die Ergebnisse der geführten Experimente demonstrieren die gezielte Selbstorganisation durch Zusammenwirken einer Vorstrukturierung langreichweitiger Ordnung und eines durch einfache thermische Aktivierung initiierten Entnetzungsvorgangs.

\section{Ausblick}

Um die genaue Ursache der unterschiedlichen Wachstumsmoden im Detail zu klären, sind systematische Untersuchungen speziell hinsichtlich des Einflusses mechanischer Spannungen erforderlich. Zu diesem Zweck ließen sich beispielsweise mittels eines Piezostellelementes definiert extrinsische uniaxiale Spannungen einbringen. Das Kornwachstum und die Textur in dünnen Schichten wird maßgeblich beeinflusst durch eine Anisotropie der treibenden Kräfte, z.B. der Ober- und Grenzflächenenergie und elastischer Verformungsenergie. Die Wahl unterschiedlicher Substrate würde sich hierbei im Wesentlichen auf die Grenzflächenenergie auswirken und somit ebenfalls einen in jüngster Zeit stark diskutierten Faktor - nämlich die Adhäsion zwischen Schicht und Substrat - abdecken.

Experimente von $\mathrm{Hu}$ et al. [Hu00] haben gezeigt, dass auch die Ionenbestrahlung zur Aktivierung des Entnetzungsprozesses genutzt werden kann. Die Dominanz viskosen Ober- 
flächenfließens, wie sie in Simulationen niederenergetischer Bestrahlung von Vauth und Mayr [Vau07] nachgewiesen wurde, dürfte dabei besonders auf rippelstrukturierten Substraten erhebliche Auswirkungen auf die Kinetik des Strukturbildungsprozesses und der Nukleationsphase besitzen. Für systematische Untersuchungen bezüglich der Skalierbarkeit und Optimierung speziell hinsichtlich der Reichweite der auftretenden atomaren Mechanismen bietet sich der Einsatz elektronenstrahllithographischer Methoden in Kombination mit der Ionenstrahlerosion an, mit deren Hilfe sich durch Abdeckung definierter Stege variabler Breite die Wellenlänge von Oberflächenkorrugationen gezielt einstellen lässt. Ein Nachteil hierbei besteht in der Beschränkung auf lokale Bereiche auf Grund der für größere Strukturen stark ansteigenden Belichtungszeit sowie in dem möglicherweise schwer zu kontrollierenden Profil der erodierten Strukturen.

Ferner können weitere temperaturabhängige Widerstandsmessungen zur genaueren Analyse des Prozessablaufs im nicht mischbaren System Ni-Ag aber auch insbesondere auf kleiner Skala zur Analyse der Strukturbildung auf den Templates dienen. Hierzu wurden bereits Vorarbeiten zur Entwicklung eines Kontaktierungskonzepts mittels zweistufiger Elektronenstrahllithographie sowie Dosistests und Stabilisierung der beiden LiftoffProzesse geleistet. 



\section{Anhang}

\section{A. XRR-Messungen an Ni/Ag-Doppellagen}

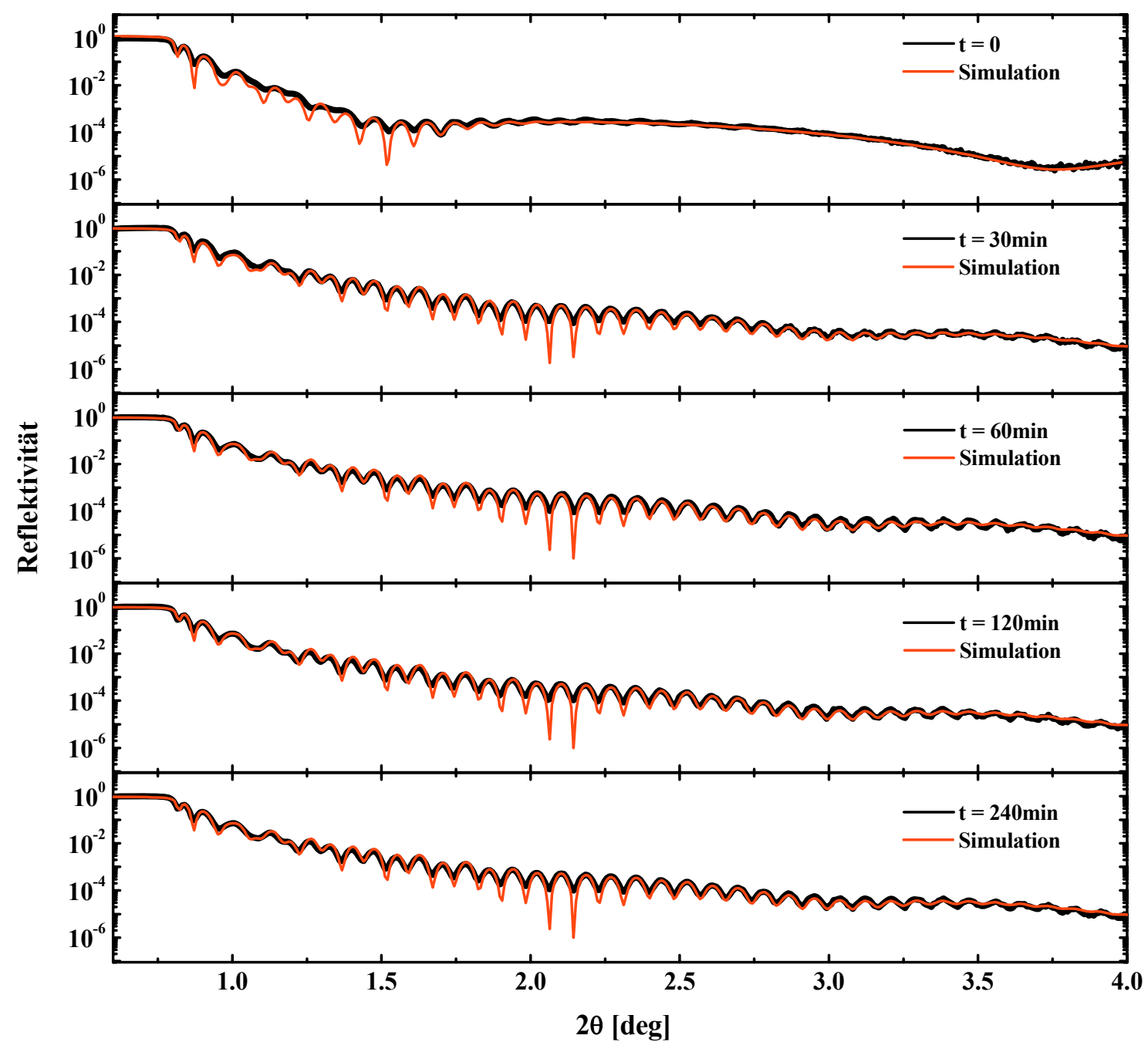

Abbildung A1: XRR-Messungen an $\mathrm{Ni}(45 \mathrm{~nm}) / \operatorname{Ag}(45 \mathrm{~nm}) / \mathrm{Zr}(4 \mathrm{~nm})-$ Schichtpaketen nach thermischer Behandlung unterschiedlicher Dauer bei $500^{\circ} \mathrm{C}$. 


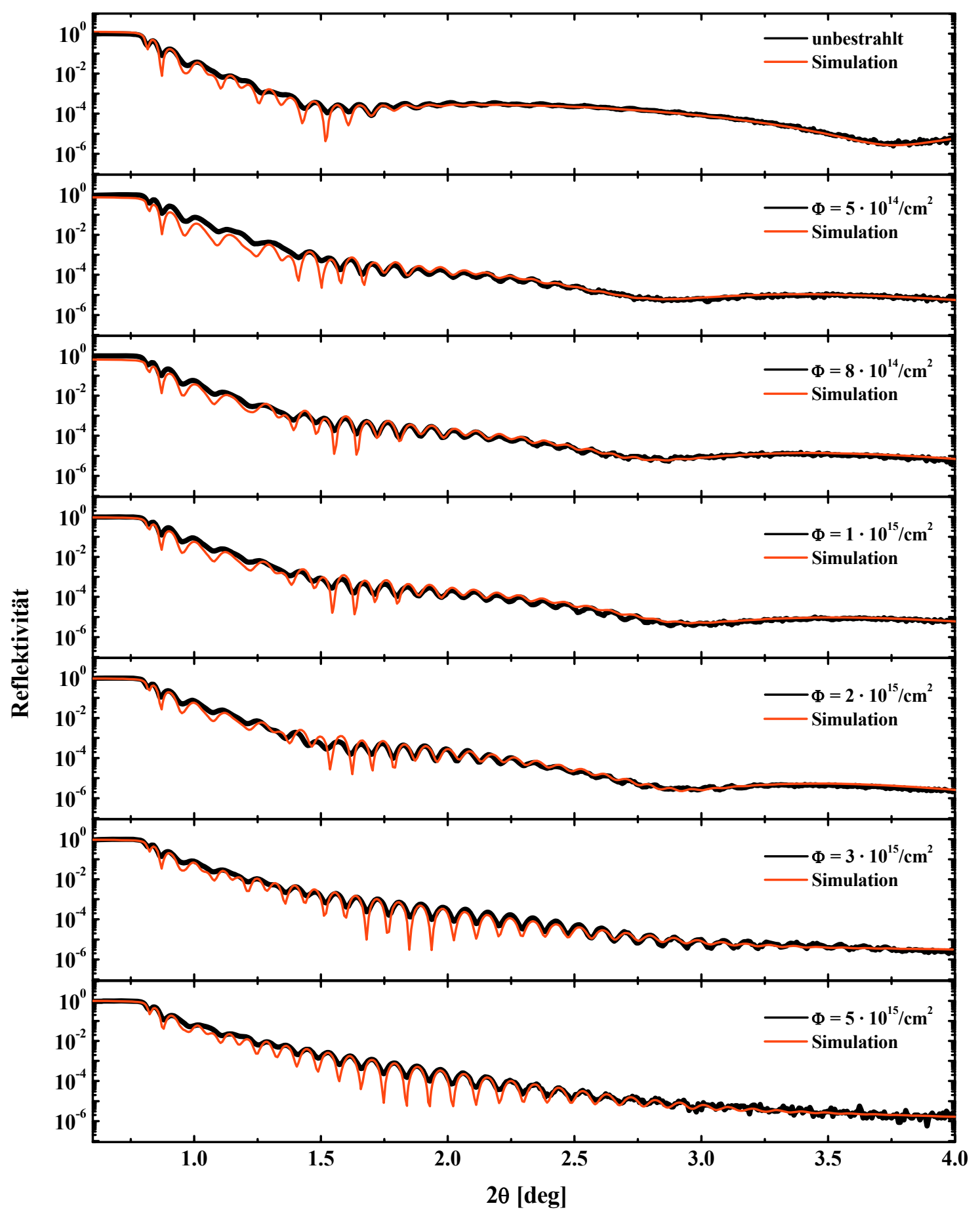

Abbildung A2: XRR-Messungen an $\mathrm{Ni}(45 \mathrm{~nm}) / \operatorname{Ag}(45 \mathrm{~nm}) / \mathrm{Zr}(4 \mathrm{~nm})$-Schichtpaketen nach $300 \mathrm{keV} \mathrm{Xe}{ }^{+}$-Bestrahlung unterschiedlicher Fluenz. 


\section{B. Parameter des Bradley-Harper-Modells}

$$
\begin{aligned}
& \Gamma_{\mathrm{x}}(\theta)=\frac{(\mathrm{a} / \alpha)^{2} \sin ^{2} \theta}{(\mathrm{a} / \alpha)^{2} \sin ^{2} \theta+(\mathrm{a} / \beta)^{2} \cos ^{2} \theta} \\
& -\frac{(\mathrm{a} / \alpha)^{2} \cos ^{2} \theta}{2(\mathrm{a} / \alpha)^{2} \sin ^{2} \theta+2(\mathrm{a} / \beta)^{2} \cos ^{2} \theta}\left(1+\frac{(\mathrm{a} / \alpha)^{4} \sin ^{2} \theta}{(\mathrm{a} / \alpha)^{2} \sin ^{2} \theta+(\mathrm{a} / \beta)^{2} \cos ^{2} \theta}\right) \\
& -\frac{(\mathrm{a} / \alpha)^{2}\left[(\mathrm{a} / \beta)^{2}-(\mathrm{a} / \alpha)^{2}\right] \sin ^{2} \theta \cos ^{2} \theta}{2\left[(\mathrm{a} / \alpha)^{2} \sin ^{2} \theta+(\mathrm{a} / \beta)^{2} \cos ^{2} \theta\right]^{2}}\left(3+\frac{(\mathrm{a} / \alpha)^{4} \sin ^{2} \theta}{(\mathrm{a} / \alpha)^{2} \sin ^{2} \theta+(\mathrm{a} / \beta)^{2} \cos ^{2} \theta}\right) \\
& \Gamma_{\mathrm{y}}(\theta)=-\frac{\beta^{2}}{\mathrm{a}^{2}}\left(\frac{1}{2}(\mathrm{a} / \alpha)^{2} \cos ^{2} \theta+\frac{(\mathrm{a} / \alpha)^{2}\left[(\mathrm{a} / \beta)^{2}-(\mathrm{a} / \alpha)^{2}\right] \sin ^{2} \theta \cos ^{2} \theta}{2(\mathrm{a} / \alpha)^{2} \sin ^{2} \theta+2(\mathrm{a} / \beta)^{2} \cos ^{2} \theta}\right) \\
& \mathrm{Y}_{0}(\theta)=\frac{\Lambda \text { Ena }}{\sqrt{2 \pi} \cdot \alpha \beta}\left[(\mathrm{a} / \alpha)^{2} \sin ^{2} \theta+(\mathrm{a} / \beta)^{2} \cos ^{2} \theta\right]^{-1 / 2} \exp \left(\frac{(\mathrm{a} / \alpha)^{4} \sin ^{2} \theta}{2\left[(\mathrm{a} / \alpha)^{2} \sin ^{2} \theta+(\mathrm{a} / \beta)^{2} \cos ^{2} \theta\right]}-\frac{\mathrm{a}^{2}}{2 \alpha^{2}}\right)
\end{aligned}
$$

$\Lambda$ ist hierbei eine materialspezifische Proportionalitätskonstante in der Beziehung zwischen lokal deponierter Leistung und der Erosionsrate, $\mathrm{n}$ bezeichnet die Atomdichte. 


\section{Oberflächenstruktur kodeponierter NiAg-Schichten}
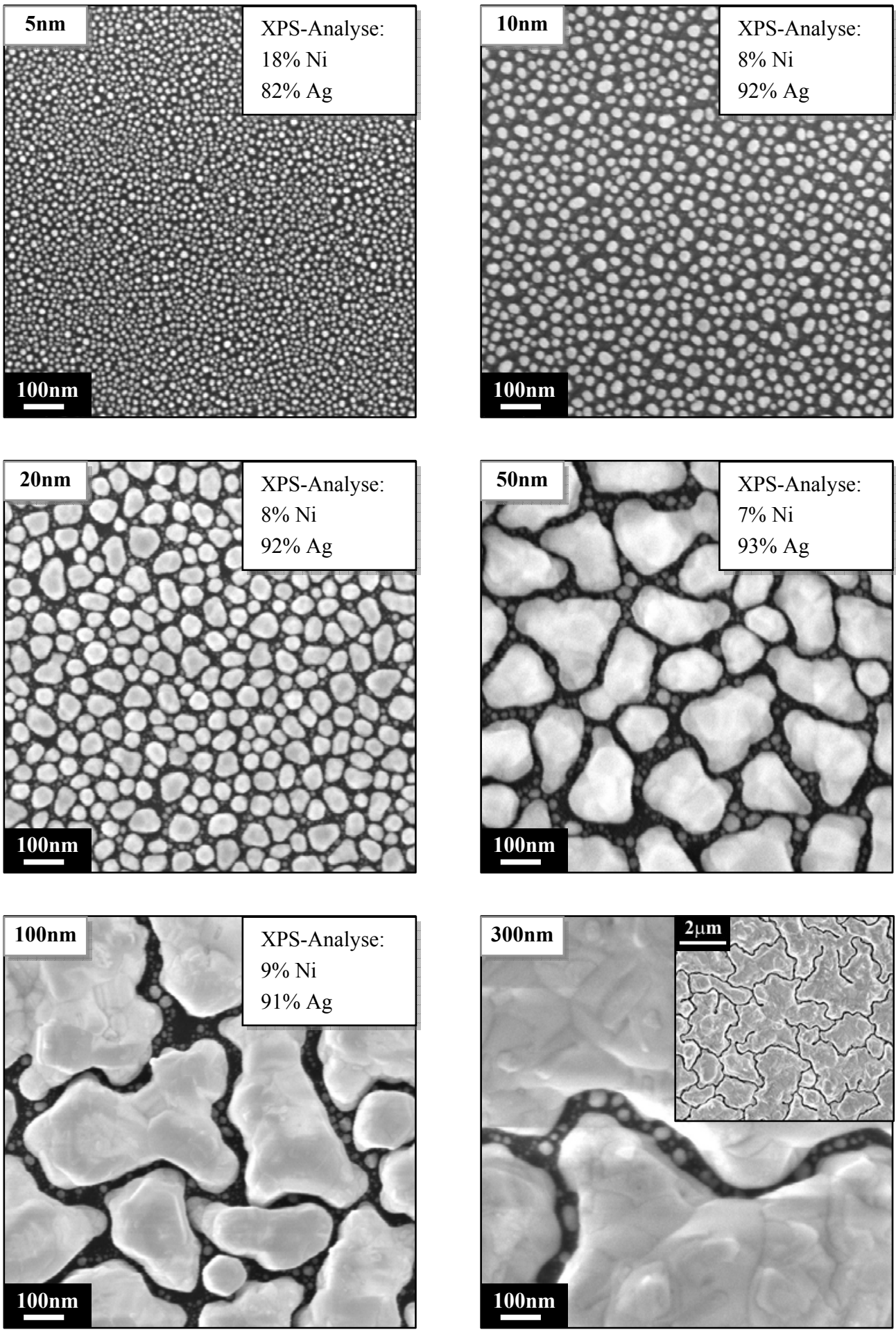

Abbildung A3: REM-Aufnahmen bei $600^{\circ} \mathrm{C}$ mittels Sputterdeposition kodeponierter NiAg-Schichten unterschiedlicher Dicke auf thermisch oxidiertem Silizium. 

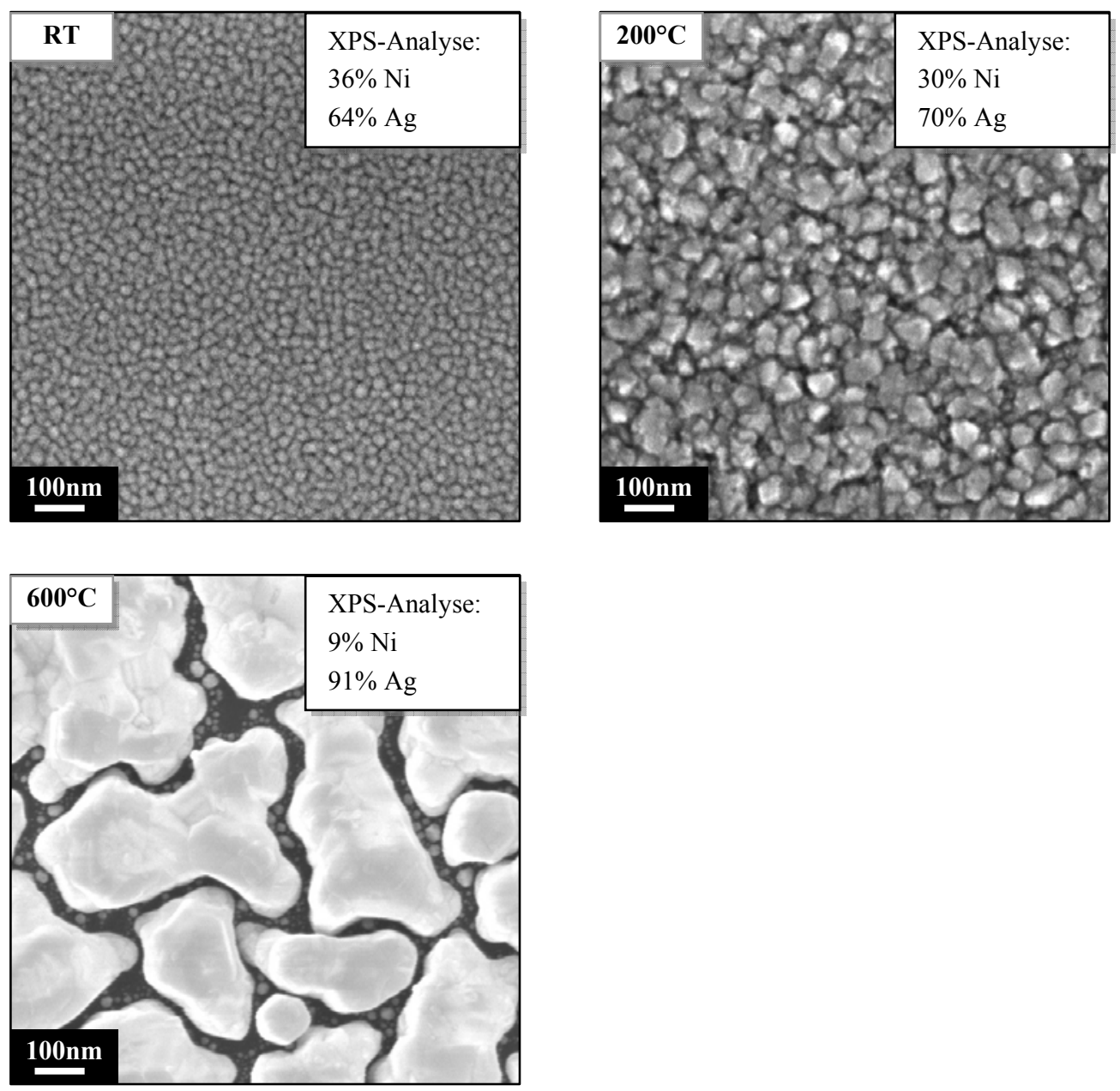

Abbildung A4: REM-Aufnahmen mittels Sputterdeposition bei unterschiedlicher Temperatur kodeponierter NiAg-Schichten einer Dicke von 100nm auf thermisch oxidiertem Silizium. 


\section{AFM-Aufnahmen der Entnetzung von Nickel auf glattem}

\section{Substrat}
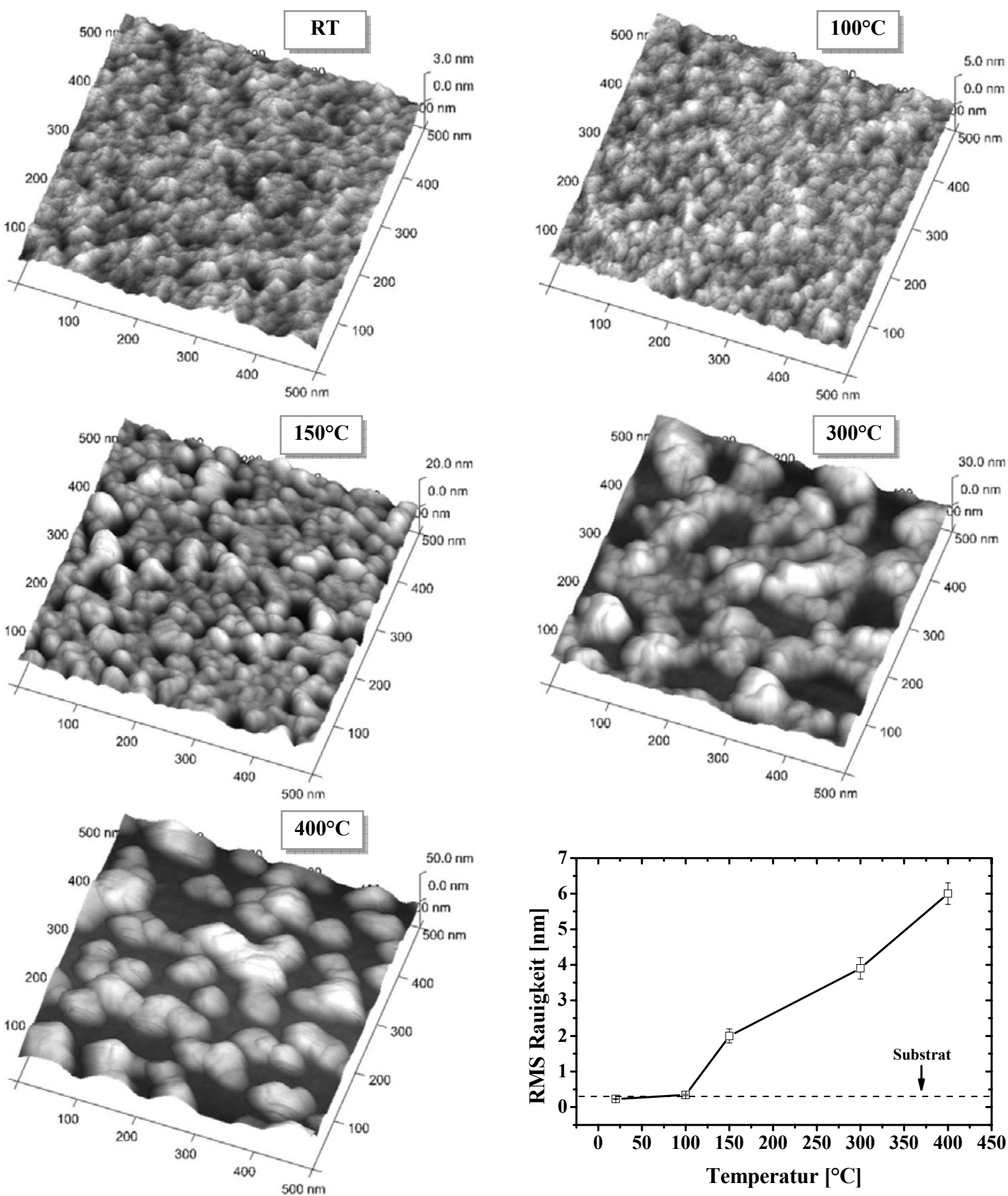

Abbildung A5: AFM-Aufnahmen der aufgedampften 5nm Ni-Schichten auf $\mathrm{SiO}_{2}$, die bei unterschiedlicher Temperatur ausgelagert wurden. Bei den vorliegenden Strukturgrößen ist zu beachten, dass die Spitzenfaltung eine erhebliche Rolle spielt. Insbesondere war es auf Grund des hohen Krümmungsradius nicht möglich, einzelne Löcher in der bei $100^{\circ} \mathrm{C}$ ausgelagerten Schicht zu untersuchen, welche in den entsprechenden REM-Aufnahmen deutlich zu erkennen sind. 


\section{E. AFM-Aufnahmen der Entnetzung von Nickel auf rip- pelstrukturiertem Substrat}
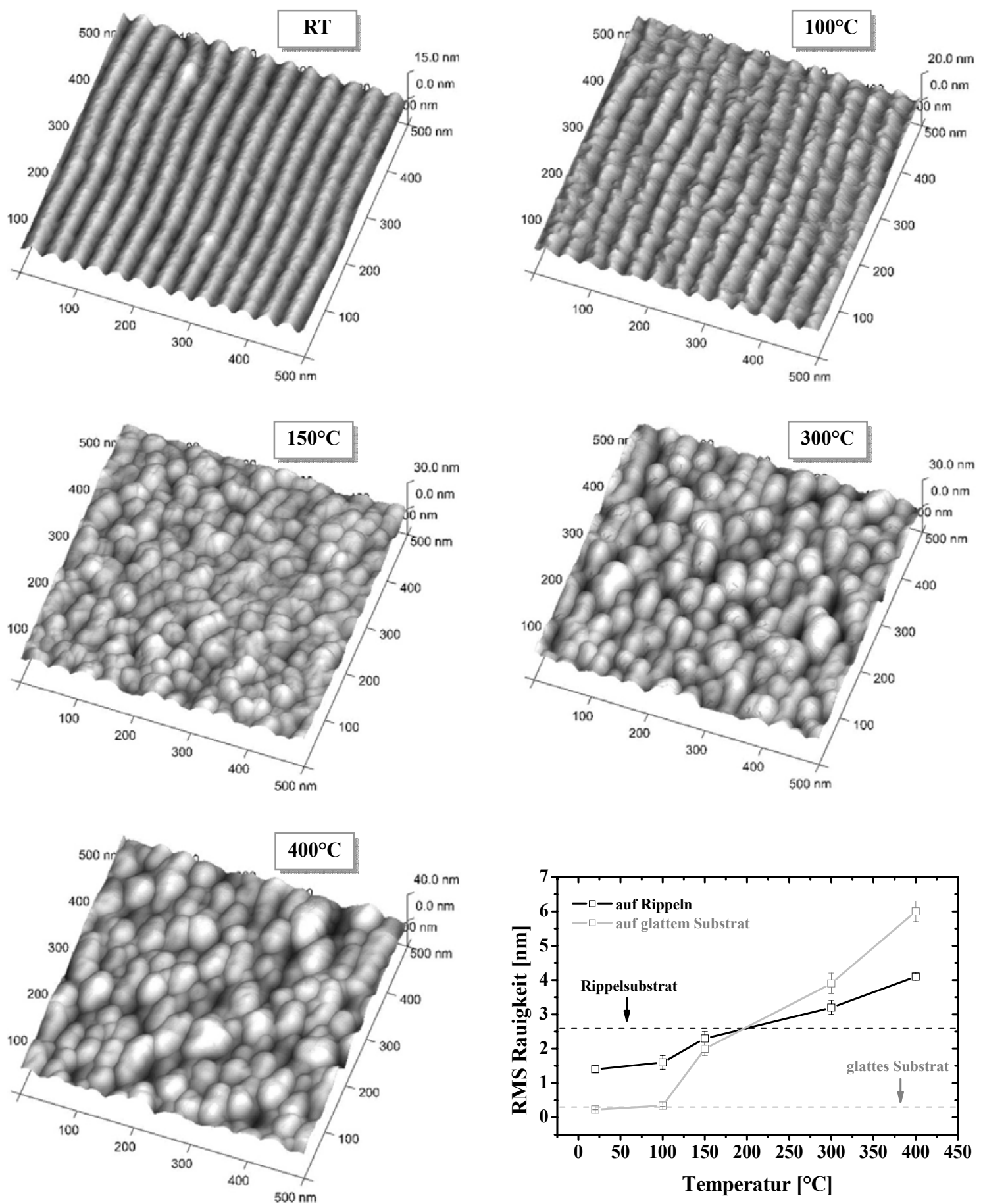

Abbildung A6: AFM-Aufnahmen der aufgedampften 5nm Ni-Schichten auf rippelstrukturiertem $\mathrm{SiO}_{2}$, die bei unterschiedlicher Temperatur ausgelagert wurden. 


\section{F. Temperaturabhängigkeit des Widerstandes einer $100 \mathrm{~nm}$ dicken Nickelschicht}

Abbildung A7 zeigt das temperaturabhängige Widerstandsverhalten einer 100nm dicken Nickelschicht, die als elektrische Zuleitung bei der Widerstandsmessung der 5nm Probeschichten verwendet und hier in Zweipunktgeometrie bei einer konstanten Heizrate von $10^{\circ} \mathrm{C} / \mathrm{min}$ gemessen wurde. Der Widerstand liegt dabei während des Aufheizvorgangs zunächst deutlich oberhalb der Abkühlkurve und nähert sich für höhere Temperaturen dieser an, was im Wesentlichen auf das thermisch aktivierte Ausheilen von Defekten und Kornwachstum zurückzuführen ist. Die Abkühlkurve zeigt ein ähnliches Verhalten, wie es für Bulk-Nickel beobachtet wird [Col58], welches sich auf Grund der Spinordnung merklich von nicht magnetischen Metallen unterscheidet und die Bestimmung der CurieTemperatur ermöglicht. In Übergangsmetallen wird der Strom vorwiegend von s-Elektronen getragen, wobei ein hoher Widerstand aus der Streuung dieser Elektronen in die freien Zustände des d-Bandes resultiert, da die schmalen d-Bänder eine hohe Zustands-

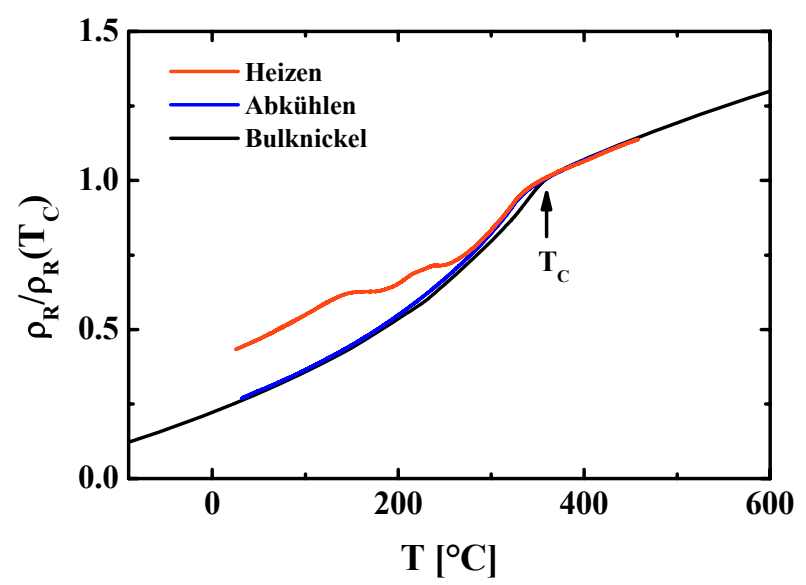

Abbildung A7: Temperaturabhängiger Widerstandsverlauf der 100nm Nickelschicht sowie von Bulk-Nickel nach Coles [Col58]. Die Widerstände sind auf den spezifischen Widerstand an der Curie-Temperatur normiert.

dichte an der Fermi-Kante besitzen. Durch die Aufspaltung der d-Subbänder im ferromagnetischen Zustand kann jedoch das Subband der Majoritätsspins unter die Fermi-Kante sinken, was eine Reduzierung der möglichen Streuzustände und damit eine Abnahme des Widerstands zur Folge hat [Mot64]. Mit zunehmender Temperatur verringert sich die Austauschaufspaltung, sodass der Widerstand unterhalb der Curie-Temperatur stark ansteigt.

Die Verschiebung der gemessenen Curie-Temperatur auf $\mathrm{T}_{\mathrm{C}}=351^{\circ} \mathrm{C}$ gegenüber dem Bulk-Wert von $\mathrm{T}_{\mathrm{C}}=361^{\circ} \mathrm{C}$ ist möglicherweise auf Verunreinigungen zurückzuführen, da 
diese Messung nicht in situ durchgeführt wurde. Untersuchungen von Morita [Mor62] haben gezeigt, dass erhöhter Restgasdruck während der Deposition die durch Widerstandsbestimmung ermittelte Curie-Temperatur von Nickel deutlich reduzieren kann. Der Einfluss der Filmdicke macht sich hingegen erst bei niedrigeren Schichtdicken bemerkbar [Bau64]. Der Fehler der Temperaturmessung liegt folglich klar unterhalb von $10^{\circ} \mathrm{C}$.

Aus dem Widerstandsverlauf während des Abkühlvorgangs wurde eine relative Widerstandsänderung bei Raumtemperatur von

$$
\left.\frac{\Delta \rho_{\mathrm{R}}(\mathrm{T})}{\Delta \mathrm{T}} \frac{1}{\rho_{\mathrm{R}}(\mathrm{T})}\right|_{\mathrm{T}=20^{\circ} \mathrm{C}}=5 \cdot 10^{-3} /{ }^{\circ} \mathrm{C}
$$

ermittelt, welche mit dem Bulk-Wert übereinstimmt und somit maßgeblich durch die Elektron-Phonon-Streuung und den Einfluss der Spinordnung bestimmt wird.

\section{G. Grenzflächenuntersuchung einer entnetzten Nickelschicht}

Zum Nachweis eines eventuell vorhandenen Wetting-Layers zwischen den Inseln nach Entnetzung eines $5 \mathrm{~nm}$ dicken Nickelfilms bei $600^{\circ} \mathrm{C}$ wurde eine mittels FIB präparierte Querschnittslamelle im TEM untersucht, mit dem unter geeigneten Bedingungen selbst bis zu Monolagen dünne Filme nachgewiesen werden können. Hierbei fällt auf, dass einige Nickelinseln teilweise in der $\mathrm{SiO}_{2}$-Schicht versunken sind (Abbildung A8). Als Ursache ist nicht auszuschließen, dass es sich um einen oberflächlichen Effekt auf der Lamelle handelt, der aus dem ionenstrahlgestützten Ausdünnverfahren zur Probenpräparation mittels $30 \mathrm{keV}$ $\mathrm{Ga}^{+}$-Ionen resultiert. Untersuchungen von $\mathrm{Hu}$ et al. [Hu02] zeigten das Einsinken von Pt-
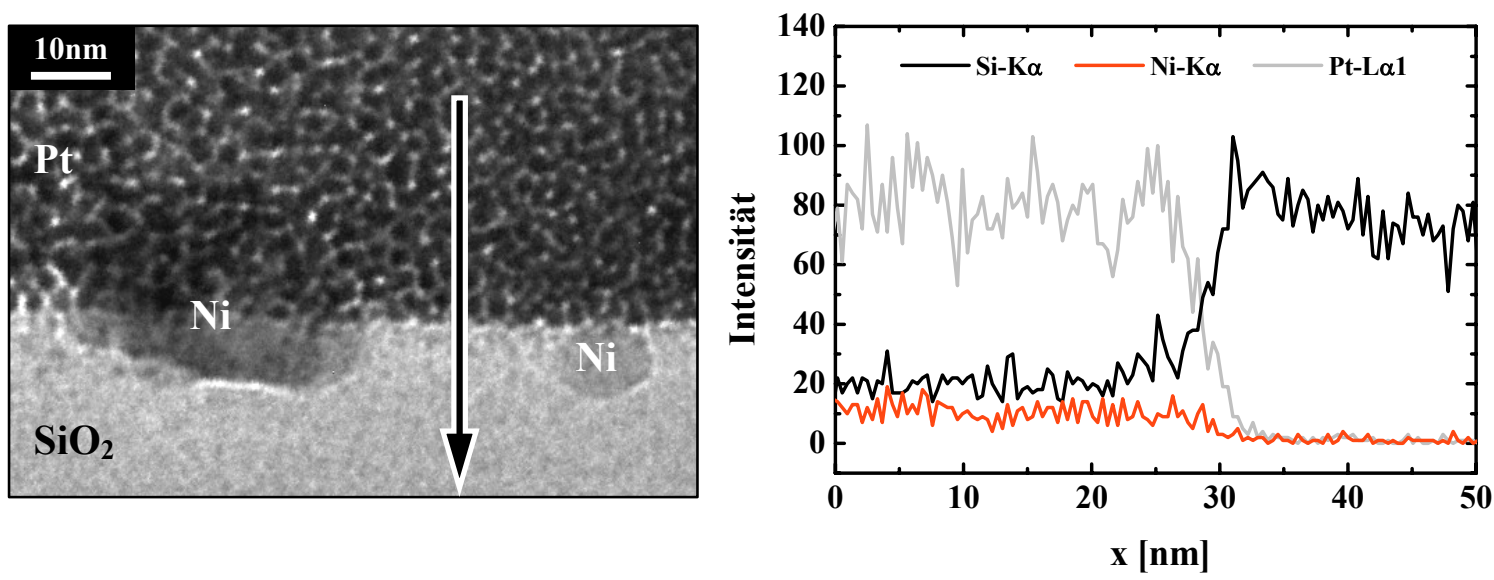

Abbildung A8: TEM-Querschnittsaufnahme einer 5nm Nickelschicht nach thermischer Behandlung von bis $\mathrm{zu} 600^{\circ} \mathrm{C}$ sowie EDX-Linienprofil entlang des Pfeils. 
Partikeln in $\mathrm{SiO}_{2}$ unter Ionenbeschuss infolge der hohen Oberflächenenergie des Metalls durch viskoses Fließen des amorphen Substrats. Jedoch ist auch das Auslösen des Effektes durch die thermische Behandlung der Probe nicht auszuschließen. Zimmermann et al. [Zim01] konnten zeigen, dass metallische Nanopartikel, deren Oberflächenenergie die des ebenfalls metallischen Substrats deutlich übersteigt, während thermischer Behandlung versinken bzw. begraben werden. Dielektrische Substrate wurden hingegen nicht verwendet. Für eine genaue Klärung der Ursache sind weitere Untersuchungen erforderlich, die jedoch nicht Thema dieser Arbeit sind.

Zur Analyse der freien Substratoberfläche zwischen den Inseln wurden EDX-Profile senkrecht zur ursprünglichen Probenoberfläche aufgezeichnet (Abbildung A8). Diese weisen jedoch keine Anzeichen für eine remanente Nickelschicht zwischen den Agglomeraten auf.

\section{H. Entnetzung einer 10nm Nickelschicht auf rippelstruktu- riertem Substrat}

Abbildung A9 zeigt die Morphologie einer bei Raumtemperatur deponierten 10nm Nickelschicht nach Auslagerung bei $300^{\circ} \mathrm{C}$ auf rippelstrukturiertem Substrat. Daran wird deutlich, dass die laterale Ausdehnung der in dem Film entstandenen Löcher wie auf glattem Substrat die Rippelwellenlänge bei Weitem übersteigt und somit die Ausbildung von Nanodrähten in benachbarten Tälern der Rippelstruktur unterminiert. Die höhere Schichtdicke von 10nm gegenüber der Rippeltiefe von $8 \mathrm{~nm}$ führt zum vollständigen Auffüllen der Täler und zur Abnahme des Einflusses der Template-Geometrie. Zwar kommt es durch die auf die Filmoberfläche übertragene Rippelgeometrie, welche selbst bei einer Schichtdicke von

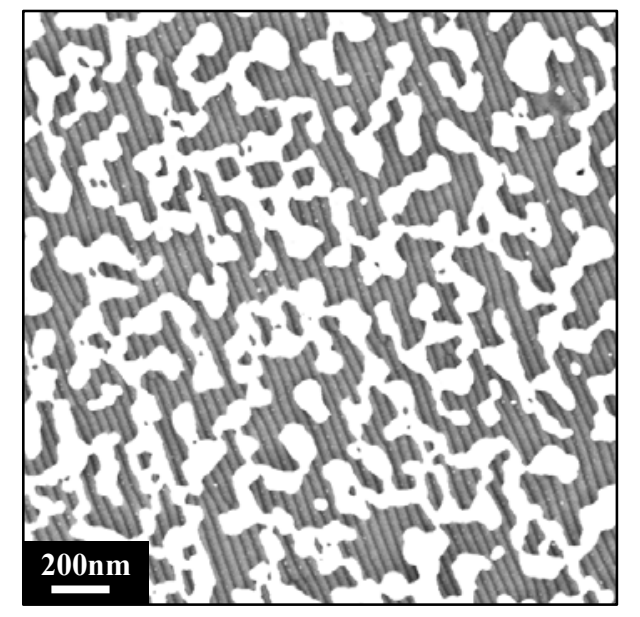

Abbildung A9: REM-Aufnahme einer bei $300^{\circ} \mathrm{C}$ ausgelagerten $10 \mathrm{~nm}$ Nickelschicht auf rippelstrukturiertem Substrat. 
$100 \mathrm{~nm}$ noch beobachtet werden konnte, auch hier vermutlich zur krümmungsinduzierten Oberflächendiffusion, diese vermag jedoch nicht, die Schicht auf den Hügeln vollständig auszudünnen und isolierte Strukturen in den Tälern zu erzeugen, sodass der herkömmliche Entnetzungsmechanismus dominiert. Die Rippel wirken indes weiterhin als Diffusionsbarrieren und die entstandenen Strukturen weisen überwiegend Kanten auf, die parallel zu den Rippeln verlaufen.

\section{Entnetzung einer sputterdeponierten Nickelschicht}

In Abbildung A10 sind REM-Aufnahmen einer bei Raumtemperatur gesputterten und anschließend für eine Stunde bei $300^{\circ} \mathrm{C}$ ausgelagerten Nickelschicht dargestellt. Die Schicht wurde, wie in Abschnitt 6.4 beschrieben, mit variierender Schichtdicke von maximal 10nm deponiert. Dabei zeigt sich ein ähnliches Entnetzungsverhalten, wie es bei aufgedampften Nickelschichten beobachtet wurde. Mit abnehmender Schichtdicke entsteht eine Vielzahl von Löchern, welche erst durch Koaleszenz isolierte Nickelinseln erzeugen. Eine fraktale Ausbreitung der Löcher wird nicht beobachtet.

\section{Schichtdicke ansteigend}
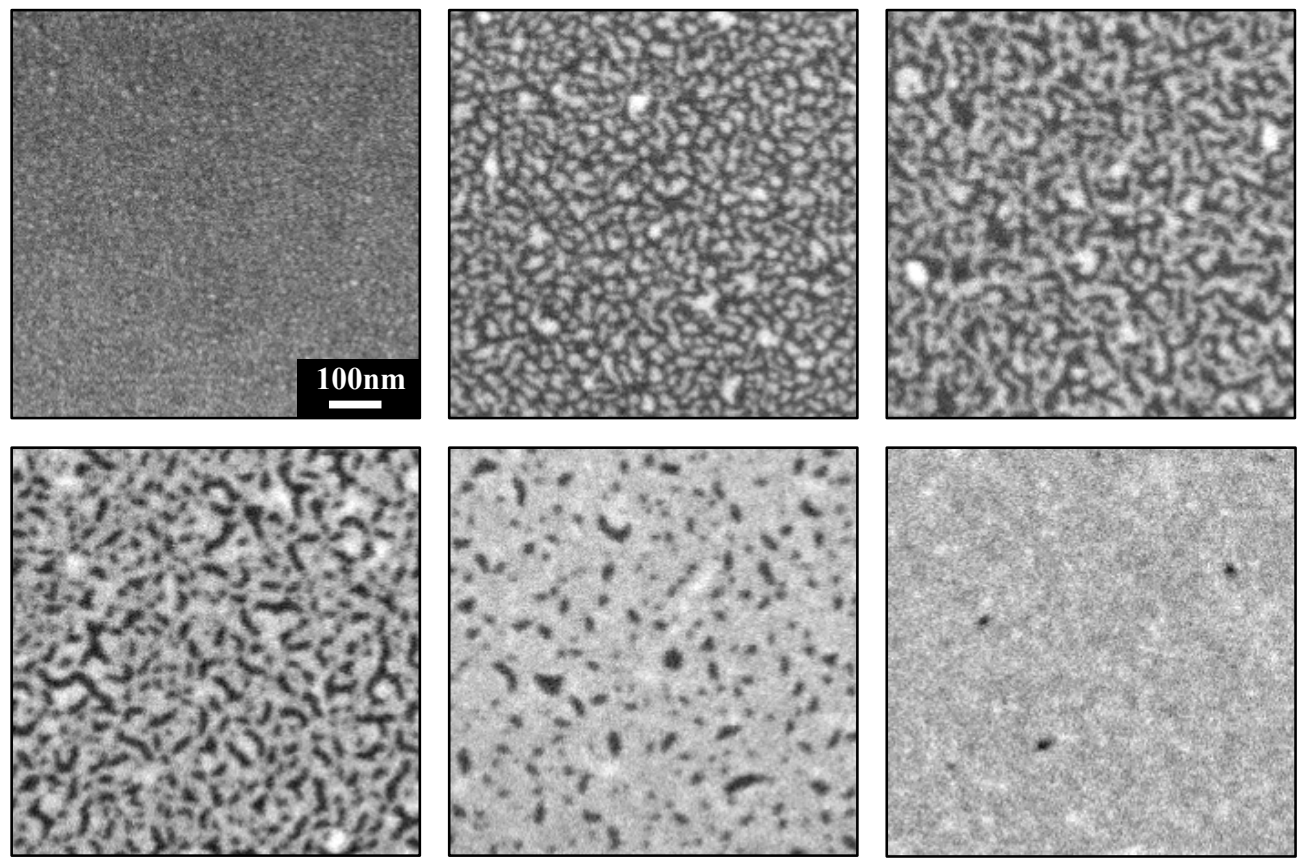

Abbildung A10: REM-Aufnahmen einer mit variierender Schichtdicke gesputterten Nickelschicht, die bei $300^{\circ} \mathrm{C}$ ausgelagert wurde. Der Maßstab aller Aufnahmen ist identisch. 



\section{Literaturverzeichnis}

[Ade92] G. A. Adegboyega, "A study of the room-temperature oxidation of thin Al films by optical transmission and sheet resistance measurements", J. Phys. III France 2 (1992) 1749

[Ale91] D. E. Alexander, G. S. Was, "The heat-of-mixing effect on ion-induced grain growth", J. Appl. Phys. 70 (1991) 1252

[Ale93] D. E. Alexander, G. S. Was, "Thermal-spike treatment of ion-induced grain growth: Theory and experimental comparison", Phys. Rev. B 47 (1993) 2983

[Alf03] T. L. Alford, L. Chen, K. S. Gadre, "Stability of silver thin films on various underlying layers at elevated temperatures", Thin Solid Films 429 (2003) 248

[Alk01] P. F. A. Alkemade, Z. X. Jiang, "Complex roughening of Si under oblique bombardment by low-energy oxygen ions", J. Vac. Sci. Technol. B 19 (2001) 1699

[Ame00] I. Ames, F. M. d'Heurle, R. E. Horstmann, "Reduction of electromigration in aluminium films by copper doping", IBM J. Res. Develop. 44 (2000) 89

[And61] J. C. Anderson, "Magnetic Anisotropy in Single-crystal Nickel Films", Proc. Phys. Soc. 78 (1961) 25

[Asa72] R. J. Asaro, W. A. Tiller, "Surface morphology development during stress corrosion cracking: Part I: via surface diffusion", Metall. Trans. 3 (1972) 1789

[Atk85] A. Atkinson, "Transport processes during the growth of oxide films at elevated temperature", Rev. Mod. Phys. 57 (1985) 437

[Atk88] H. V. Atkinson, "Theories of normal grain growth in pure single phase systems", Acta Metall. 36 (1988) 469

[Ave86] R. S. Averback, D. Peak, L. J. Thompson, "Ion-Beam Mixing in Pure and in Immiscible Copper Bilayer Systems", Appl. Phys. A 39 (1986) 59

[Ave88] R. S. Averback, T. Diaz De La Rubia, R. Benedek, "Dynamics and structure of energetic displacement cascades", Nucl. Instrum. Meth. B 33 (1988) 693 
[Bar02] E. V. Barnat, D. Nagakura, P.-I. Wang, T.-M. Lu, "Real time resistivity measurements during sputter deposition of ultrathin copper films", J. Appl. Phys. 91 (2002) 1667

[Bas76] D. W. Bassett, "Migration of platinum Adatom clusters on tungsten (110) surfaces", J. Phys. C 9 (1976) 2491

[Bau64] H. J. Bauer, K. Geissler, W. Juergens, "Zur Curie-Temperatur dünner Nickelschichten", Z. Phys. 180 (1964) 91

[Bin89] G. Binasch, P. Grünberg, F. Saurenbach, W. Zinn, "Enhanced magnetoresistance in layered magnetic structures with antiferromagnetic interlayer exchange", Phys. Rev. B 39 (1989) 4828

[Bis96] J. Bischof, D. Scherer, S. Herminghaus, P. Leiderer, "Dewetting Modes of Thin Metallic Films: Nucleation of Holes and Spinodal Dewetting", Phys. Rev. Lett. 77 (1996) 1536

[Bla94] J. A. Blackman, B. L. Evans, A. I. Maaroof, "Analysis of island-size distributions in ultrathin metallic films", Phys. Rev. B 49 (1994) 13863

[Boe88] F. R. de Boer, R. Boom, W. C. M. Mattens, A. R. Miedema, A. K. Niessen, "Cohesion in Metals", edited by F. R. de Boer and D. Pettifor, (Elsevier, Amsterdam, 1988)

[Bo194] W. Bolse, "Ion-beam induced atomic transport through bi-layer interfaces of low- and medium-Z metals and their nitrides", Mater. Sci. Eng. R 12 (1994) 53

[Bou07] M. Bouville, "Effect of grain shape on the agglomeration of polycrystalline thin films", Appl. Phys. Lett. 90 (2007) 061904

[Bra66] R. H. Brandon, F. J. Bradshaw, R. A. E. Res. Rep. No. 66095 (1966)

[Bra88] R. M. Bradley, J. M. E. Harper, "Theory of ripple topography induced by ion bombardment", J. Vac. Sci. Technol. A 6 (1988) 2390

[Bra04] A. Bracchi, "Structural and Magnetic Properties of the Glass-Forming Alloy $\mathrm{Nd}_{60} \mathrm{Fe}_{30} \mathrm{Al}_{10}$ ", Dissertation, Universität Göttingen (2004)

[Bro90] F. Brochard-Wyart, J. Daillant, "Drying of solids wetted by thin liquid films", Can. J. Phys. 68 (1990) 1084

[Bro94] F. Brochard-Wyart, P.-G. de Gennes, H. Hervert, C. Redon, "Wetting and Slippage of Polymer Melts on Semi-ideal Surfaces", Langmuir 10 (1994) 1566

[Bro05] A.-D. Brown, J. Erlebacher, W. L. Chan, E. Chason, "Transient Topographies of Ion Patterned Si(111)", Phys. Rev. Lett. 95 (2005) 056101 
[Bro05a] A.-D. Brown, J. Erlebacher, "Temperature and fluence effects on the evolution of regular surface morphologies on ion-sputtered Si(111)", Phys. Rev. B 72 (2005) 075350

[Cha93] E. Chason, M. Mayer, "Low energy ion bombardment induced roughening and smoothing of $\mathrm{SiO}_{2}$ surfaces", Appl. Phys. Lett. 25 (1993) 363

[Cha07] W. L. Chan, E. Chason, "Making waves: Kinetic processes controlling surface evolution during low energy ion sputtering", J. Appl. Phys. 101 (2007) 121301

[Che02] R. Cheng, C. N. Borca, N. Pilet, B. Xu, L. Yuan, B. Doudin, S. H. Liou, P. A. Dowben, "Oxidation of metals at the chromium oxide interface", Appl. Phys. Lett. 81 (2002) 2109

[Chi02] T. K. Chini, M. K. Sanyal, S. R. Bhattacharyya, "Energy-dependent wavelength of the ion-induced nanoscale ripple", Phys. Rev. B 66 (2002) 153404

[Chi03] T. K. Chini, F. Okuyama, M. Tanemura, K. Nordlund, "Structural investigation of keV Ar-ion-induced surface ripples in Si by cross-sectional transmission electron microscopy", Phys. Rev. B 67 (2003) 205403

[Col58] B. R. Coles, "Spin-disorder Effects in the Electrical Resistivities of Metals and Alloys", Adv. Phys. 7 (1958) 40

[Cre96] A. Crespo-Sosa, P. Schaaf, W. Bolse, K.-P. Lieb, "Irradiation effects in AgFe bilayers: Ion-beam mixing, recrystallization, and surface roughening", Phys. Rev. B 53 (1996) 14795

[Cre03] A. Crespo-Sosa, M. Munoz, J.-C. Cheang-Wong, A. Oliver, J. M. Saniger, J. G. Banuelos, "Thermal spikes in $\mathrm{Ag} / \mathrm{Fe}$ and $\mathrm{Cu} / \mathrm{Fe}$ ion beam mixing", Mater. Sci. Eng. B 100 (2003) 297

[Cue95] R. Cuerno, A.-L. Barabási, "Dynamic Scaling of Ion-Sputtered Surfaces", Phys. Rev. Let. 74 (1995) 4746

[Cul78] B. D. Cullity, "Elements of X-Ray Diffraction", (Addison-WesleyPublishing, 1978)

[Dan00] R. Dannenberg, E. A. Stach, J. R. Groza, B. J. Dresser, "In-situ TEM observations of abnormal grain growth, coarsening, and substrate de-wetting in nanocrystalline Ag thin films", Thin Solid Films 370 (2000) 54

[Dat01] A. Datta, Y.-R. Wu, Y. L. Wang, "Real-time observation of ripple structure formation on diamond surface under focused ion-beam bombardment", Phys. Rev. B 63 (2001) 125407

[Die05] S. Dietrich, M. N. Popescu, M. Rauscher, "Wetting on structured substrates", J. Phys.: Condens. Matter 17 (2005) S577 
[Dij01] S. van Dijken, D. de Bruin, B. Poelsema, "Kinetic Physical Etching for Versatile Novel Design of Well Ordered Self-Affine Nanogrooves", Phys. Rev. Lett. 86 (2001) 4608

[Dor06] E. Dornel, J.-C. Barbé, F. de Crécy, G. Lacolle, "Surface diffusion dewetting of thin solid films: Numerical method and application to $\mathrm{Si} / \mathrm{SiO}_{2}$ ", Phys. Rev. B 73 (2006) 115427

[Dus79] E. B. Dussan, "Liquids on solid surfaces: Static and dynamic contact lines", Ann. Rev. Fluid Mech. 11 (1979) 371

[Ent05] S. Entani, M. Kiguchi, S. Ikeda, K. Saiki, "Magnetic properties of ultrathin cobalt films on $\mathrm{SiO}_{2}$ substrates", Thin Solid Films 493 (2005) 221

[Erd04] Z. Erdélyi, M. Sladecek, L.-M. Stadler, I. Zizak, G. A. Langer, M. KisVarga, D. L. Beke, B. Sepiol, "Transient Interface Sharpening in Miscible Alloys", Science 306 (2004) 1913

[Eri99] M. Eriksson, L. Olsson, U. Helmersson, R. Erlandsson, L.-G. Ekedahl, "Morphology changes of thin $\mathrm{Pd}$ films grown on $\mathrm{SiO}_{2}$ : influence of adsorbates and temperature", Thin Solid Films 342 (1999) 297

[Fac99] S. Facsko, T. Dekorsy, C. Koerdt, C. Trappe, H. Kurz, A. Vogt, H. L. Hartnagel, "Formation of Ordered Nanoscale Semiconductor Dots by Ion Sputtering", Science 285 (1999) 1551

[Far05] M. Farle, "Magnetic Nanoparticles", 36. Ferienkurs, Magnetism goes Nano, (Forschungszentrum Jülich, 2005)

[Fla01] D. Flamm, F. Frost, D. Hirsch, "Evolution of surface topography of fused silica by ion beam sputtering", Appl. Surf. Sci. 179 (2001) 95

[Fro00] F. Frost, A. Schindler, F. Bigl, "Roughness Evolution of Ion Sputtered Rotating InP Surfaces: Pattern Formation and Scaling Laws", Phys. Rev. Lett. 85 (2000) 4116

[Fro04] F. Frost, R. Fechner, D. Flamm, B. Ziberi, W. Frank, A. Schindler, "Ion beam assisted smoothing of optical surfaces", Appl. Phys. A 78 (2004) 651

[Fuh04] C. Fuhse, H.-U. Krebs, S. Vitta, G. A. Johansson, "Interface quality and thermal stability of laser-deposited metal/MgO multilayers", Appl. Opt. 43 (2004) 6265

[Ful02] B. Fultz, J. M. Howe, "Transmission Electron Microscopy and Diffractometry of Materials", 2. Auflage, (Springer Verlag, Berlin, 2002)

[Gad05] P. R. Gadkari, A. P. Warren, R. M. Todi, R. V. Petrova, K. R. Coffey, "Comparison of the agglomeration behavior of thin metallic films on $\mathrm{SiO}_{2}$ ", J. Vac. Sci. Technol. A 23 (2005) 1152 
[Gao91] H. Gao, "Stress Concentration at Slightly Undulating Surfaces", J. Mech. Phys. Solids 39 (1991) 443

[Gau99] H. Gau, S. Herminghaus, P. Lenz, R. Lipowsky, "Liquid Morphologies on Structured Surfaces: From Microchannels to Microchips", Science 283 (1999) 46

[Gei96] H. Geisler, "Stabilität von dünnen amorphen Metallschichten in Multilagen mit großer Grenzflächen-Fehlanpassung", Dissertation, Universität Augsburg (1996)

[Gla01] A. Glaeser, "Model Studies of Rayleigh Instabilities via Microdesigned Interfaces", Interface Sci. 9 (2001) 65

[Gre73] E. A. Grey, G. T. Higgins, "Solute limited grain boundary migration: A rationalisation of grain growth", Acta Metall. 21 (1973) 309

[Gri86] M. Y. Grinfeld, " Instability of the separation boundary between a nonhydrostatically stressed elastic body and a melt", Sov. Phys. Dokl. 31 (1986) 831

[Hab99] S. Habenicht, W. Bolse, K. P. Lieb, K. Reimann, U. Geyer, "Nanometer ripple formation and self-affine roughening of ion-beam-eroded graphite surfaces", Phys. Rev. B 60 (1999) R2200

[Han75] N. B. Hannay, "Treatise on Solid State Chemistry", (Plenum Press, New York, 1975)

[Hat01] A. Hatzor, P. S. Weiss, "Molecular Rulers for Scaling Down Nanostructures", Science 291 (2001) 1019

[Haz04] S. Hazra, T. K. Chini. M. K. Sanyal, J. Grenzer, U. Pietsch, "Ripple structure of crystalline layers in ion-beam-induced Si wafers", Phys. Rev. B 70 (2004) 121307

[Her51] C. Herring, "Physics of Powder Metallurgy", edited by Walter E. Kingston, ( McGraw-Hill Book, Company, Inc., New York, 1951)

[Her98] S. Herminghaus, K. Jacobs, K. Mecke, J. Bischof, A. Fery, M. Ibn-Elhaj, S. Schlagowski, "Spinodal Dewetting in Liquid Crystal and Liquid Metal Films", Science 282 (1998) 916

[Hu00] X. Hu, D. G. Cahill, R. S. Averback, "Nanoscale pattern formation in Pt thin films due to ion-beam-induced dewetting", Appl. Phys. Lett. 76 (2000) 3215

[Hu02] X. Hu, D. G. Cahill, R. S. Averback, "Burrowing of Pt nanoparticles into $\mathrm{SiO}_{2}$ during ion-beam irradiation", J. Appl. Phys. 92 (2002) 3995

[Hum81] R. E. Hummel, R. T. DeHoff, S. Matts-Goho, W. M. Goho, "Thermal grooving, thermotransport and electrotransport in doped and undoped thin gold films", Thin Solid Films 78 (1981) 1 
[Iij93] S. Iijima, T. Ichihashi, "Single-shell carbon nanotubes of 1-nm diameter", Nature 363 (1993) 603

[Ish03] Y. Ishikawa, Y. Imai, H. Ikeda, M. Tabe, "Pattern-induced alignment of silicon islands on buried oxide layer of silicon-on-insulator structure", Appl. Phys. Lett. 83 (2003) 3162

[Jac90] U. Jacob, J. Vancea, H. Hoffmann, "Surface-roughness contributions to the electrical resistivity of polycrystalline metal films", Phys. Rev. B 41 (1990) 11852

[Jac98] K. Jacobs, S. Herminghaus, K. R. Mecke, "Thin Liquid Polymer Films Rupture via Defects", Langmuir 14 (1998) 965

[Jia98] Z. X. Jiang, P. F. A. Alkemade, "The complex formation of ripples during depth profiling of Si with low energy, grazing oxygen beams", Appl. Phys. Lett. 73 (1998) 315

[Jir90] E. Jiran, C. V. Thompson, "Capillary Instabilities in Thin Films", J. Electron. Mater. 19 (1990) 1153

[Joh85] W. L. Johnson, Y. T. Cheng, M. van Rossum, M.-A. Nicolet, "When is Thermodynamics Relevant to Ion-Induced Atomic Rearrangements in Metals?", Nucl. Instrum. Meth. B 7/8 (1985) 657

[Kak06] K. Kakehi, S. Noda, S. Chiashi, S. Maruyama, "Supported Ni catalysts from nominal monolayer grow single-walled carbon nanotubes", Chem. Phys. Lett. 428 (2006) 381

[Kar04] P. Karmakar, D. Ghose, "Ion beam sputtering induced ripple formation in thin metal films", Surf. Sci. 554 (2004) L101

[Kar07] S. Karim, M. E. Toimil-Molares, W. Ensinger, A. G. Balogh, T. W. Cornelius, E. U. Khan, R. Naumann, "Influence of crystallinity on the Rayleigh instability of gold nanowires", J. Phys. D: Appl. Phys. 40 (2007) 3767

[Kau06] M. Djordjevic Kaufmann, "Magnetization dynamics in all-optical pumpprobe experiments: spin-wave modes and spin-current damping", Dissertation, Universität Göttingen (2006)

[Kim02] H. C. Kim, T. L. Alford, D. R. Allee, "Thickness dependence on the stability of silver thin films", Appl. Phys. Lett. 81 (2002) 4287

[Kis56] H. E. Kissinger, "Variation of the peak temperature with heating rate in Differential Thermal Analysis", J. Res. Natl. Bur. Stand., Sect. A 57 (1956) 217

[Kli01] L. Klinger, E. Rabkin, "Effects of Surface Anisotropy on Grain Boundary Grooving", Interface Science 9 (2001) 55

[Kra03] B. Krause, A. C. Dürr, F. Schreiber, H. Dosch, O. H. Seeck, "Thermal stability and partial dewetting of crystalline organic thin films: 3,4,9,10- 
perylenetetracarboxylic dianhydride on $\operatorname{Ag}(111) "$, J. Chem. Phys. 119 (2003) 3429

[Kre95] H. U. Krebs, Y. Luo, M. Störmer, A. Crespo, P. Schaaf, W. Bolse, "Structural changes during Ar-ion irradiation of laser-deposited Fe/Ag multilayers", Appl. Phys. A: Mater. Sci. Process. 61 (1995) 591

[Kro02] K. Kroy, G. Sauermann, H. Herrmann, "Minimal Model for Sand Dunes", Phys. Rev. Lett. 88 (2002) 054301

[Kro05] K. Kroy, S. Fischer, B. Obermayer, "The shape of barchan dunes" J. Phys.: Condens. Matter 17 (2005) 1229

[Kum83] V. K. Kumikov, Kh. B. Khokonov, "On the measurement of surface free energy and surface tension of solid metals", J. Appl. Phys. 54 (1983) 1346

[Kwo03] J.-Y. Kwon, T.-S. Yoon, K.-B. Kim, S.-H. Min, "Comparison of the agglomeration behavior of $\mathrm{Au}$ and $\mathrm{Cu}$ films sputter deposited on silicon dioxide", J. Appl. Phys. 93 (2003) 3270

[Lah69] S.K. Lahiri, O.C. Wells, "Reversible Hillock Growth in Thin Films", Appl. Phys. Lett., 15 (1969) 234

[Len92] B. Lengeler, "Röntgenreflexion und diffuse Streuung an Grenzflächen", in 23. IFF Ferienkurs der KFA Jülich (1992)

[Leu04] F. Leuenberger, "Elemental resolved magnetism of Gadoliniumnitride layers and GdN/Fe multilayers", Dissertation, Universität Göttingen (2004)

[Lop02] L. Lopez-Diaz, L. Torres, "Transition from ferromagnetism to superparamagnetism on the nanosecond time scale", Phys. Rev. B 65 (2002) 224406

[Lu07] A.-H. Lu, E. L. Salabas, F. Schüth, "Magnetische Nanopartikel: Synthese, Stabilisierung, Funktionalisierung und Anwendung", Angew. Chem. 119 (2007) 1242

[Mac92] S. W. MacLaren, J. E. Baker, N. L. Finnegan, C. M. Loxton, "Surface roughness development during sputtering of GaAs and InP: Evidence for the role of surface diffusion in ripple formation and sputter cone development", J. Vac. Sci. Technol. A 10 (1992), 468

[Mak02] M. A. Makeev, R. Cuerno, A.-L. Barabási, "Morphology of ionsputtered surfaces", Nucl. Instrum. Meth. B 197 (2002) 185

[Mas86] T. B. Massalski, "Binary Alloy Phase Diagram", (American Society for Metals, Metal Parks, Ohio, 1986)

[May70] A. F. Mayadas, M. Shatzkes, "Electrical-Resistivity Model for Polycrystalline Films: the Case of Arbitrary Reflection at External Surfaces", Phys. Rev. B 1 (1970) 1382 
[May92] J. T. Mayer, R. F. Lin, E. Garfunkel, "Surface and bulk diffusion of adsorbed nickel on ultrathin thermally grown silicon dioxide", Surf. Sci. 265 (1992) 102

[May00] S. G. Mayr, "Wachstum amorpher Schichten: Vergleich von Experiment und Simulation im Bereich Oberflächenrauhigkeit und mechanische Spannungen", Dissertation, Universität Göttingen (2000)

[May01] S. G. Mayr, R. S. Averback, "Surface smoothing of rough amorphous films by irradiation-induced viscous flow", Phys. Rev. Lett. 87 (2001) 196106

[May03] S. G. Mayr, R. S. Averback, "Effect of ion bombardment on stress in thin metal films", Phys. Rev. B 68 (2003) 214105

[May03a] S. G. Mayr, Y. Ashkenazy, K. Albe, R. S. Averback, "Mechanisms of Radiation-Induced Viscous Flow: Role of Point Defects", Phys. Rev. Lett. 90 (2003) 055505

[May03b] S. G. Mayr, R. S. Averback, "Evolution of morphology in nanocrystalline thin films during ion irradiation", Phys. Rev. B 68 (2003) 075419

[May05] T. M. Mayer, D. P. Adams, M. J. Vasile, K. M. Archuleta, "Morphology evolution on diamond surfaces during ion sputtering", J. Vac. Sci. Technol. A 23 (2005) 1579

[Moc05] U. Mock, T. Michel, C. Tropea, I. Roisman, J. Rühle, "Drop impact on chemically structured arrays", J. Phys.: Condens. Matter 17 (2005) S595

[Mol04] M. E. Toimil Molares, A. G. Balogh, T. W. Cornelius, R. Neumann, C. Trautmann, "Fragmentation of nanowires driven by Rayleigh instability", Appl. Phys. Lett. 85 (2004) 5337

[Moo95] J. S. Moodera, L. R. Kinder, T. M. Wong, R. Meservey, "Large Magnetoresistance at Room Temperature in Ferromagnetic Thin Film Tunnel Junctions", Phys. Rev. Lett. 74 (1995) 3273

[Mor62] N. Morita, "Curie Temperature of Evaporated Nickel Films", J. Phys. Soc. Jpn. 17 (1962) 1155

[Mos05] M. Moseler, P. Gumbsch, C. Casiraghi, A. C. Ferrari, J. Robertson, "The Ultrasmoothness of Diamond-like Carbon Surfaces", Science 309 (2005) 1545

[Mot64] N. F. Mott, "Electrons in Transition Metals", Adv. Phys. 13 (1964) 325

[Mug05] F. Mugele, A. Klingner, J. Buehrle, D. Steinhauser, S. Herminghaus, "Electrowetting: a convenient way to switchable wettability patterns", J. Phys.: Condens. Mater. 17 (2005) S559

[Mul57] W. W. Mullins, "Theory of Thermal Grooving", J. Appl. Phys. 28 (1957) 333 
[Mul58] W. W. Mullins, "The effect of thermal grooving on grain boundary motion", Acta Metall. 6 (1958) 414

[Mul59] W. W. Mullins, "Flattening of a Nearly Plane Solid Surface due to Capillarity", J. Appl. Phys. 30 (1959) 77

[Nag83] V. K. Nagesh, J. A. Pask, "Wetting of nickel by silver", J. Mater. Sci. 18 (1983) 2665

[Nav62] M. Navez, C. Sella, D. Chaperot, "Microscopie electronique-étude de lattaque du verrepar bombardement ionique", C. R. J. Phys. 254 (1962) 240

[Neu96] M. Neubauer, K. P. Lieb, P. Schaaf, M. Uhrmacher, "Ion-beam mixing of $\mathrm{Ag} / \mathrm{Fe}$ and $\mathrm{In} / \mathrm{Fe}$ layers studied by hyperfine techniques", Phys. Rev. B 53 (1996) 10237

[Neu99] M. Neubauer, K. P. Lieb, M. Uhrmacher, P. Wodniecki, "The size of thermal spikes in xenon-irradiated $\mathrm{Ag} / \mathrm{Fe}$ bilayers", Hyperfine Interact. 120/121 (1999) 331

[Nev80] L. Névot, P. Croce, "Characterisation of surfaces by grazing X-ray reflection. Application to the study of polishing some silicate glasses", Rev. Phys. Appl. 15 (1980) 761

[Nic65] F. A. Nichols, W. W. Mullins, "Surface- (interface-) and volume-diffusion contributions to morphological changes driven by capillarity", Trans. Met. Soc. AIME 233 (1965) 1840

[No191] T. Nolan, R. Beyers, R. Sinclair, "Evidence for a grain boundary grooving model of agglomeration in polycrystalline $\mathrm{TiSi}_{2}$ thin films", Mat. Res. Soc. Symp. Proc. 202 (1991) 95

[Odi94] C. Odin, J. P. Aimé, Z. El Kaakour, T. Bouhacina, "Tip's finite size effects on atomic force microscopy in the contact mode: simple geometrical considerations for rapid estimation of apex radius and tip angle based on the study of polystyrene latex balls", Surf. Sci. 317 (1994) 321

[Oec73] H. Oechsner, "Untersuchung zur Festkörperzerstäubung bei schiefwinkligem Ionenbeschuß polykristalliner Metalloberflächen im Energiebereich um 1 keV", Z. Physik 261 (1973) 37

[Ohl01] H. Ohldag, T. J. Regan, J. Stöhr, A. Scholl, F. Nolting, J. Lüning, C. Stamm, S. Anders, R. L. White, "Spectroscopic Identification and Direct Imaging of Interfacial Magnetic Spins", Phys. Rev. Lett. 87 (2001) 247201

[Ohr02] M. Ohring, "Materials Science of Thin Films, Deposition and Structure", Second Edition (San Diego, CA, Academic Press, 2002)

[Orc62] S. E. Orchard, "On surface levelling in viscous liquids and gels", Appl. Sci. Res. 11A (1962) 451 
[Oro97] A. Oron, S. H. Davis, S. G. Bankoff, "Long-scale evolution of thin liquid films", Rev. Mod. Phys. 69 (1997) 931

[Par54] L. G. Parratt, "Surface Studies of Solids by Total Reflection of X-Rays", Phys. Rev. 95 (1954) 359

[Par65] J. R. Parsons, "Conversion of Crystalline Germanium to Amorphous Germanium by Ion Bombardment", Phil. Mag. 12 (1965) 1159

[Par99] S. Park, B. Kahng, H. Jeong, A.-L. Barabasi, "Dynamics of Ripple Formation in Sputter Erosion: Nonlinear Phenomena", Phys. Rev. Lett. 83 (1999) 3486

[Pas64] D. W. Pashley, M. J. Stowell, M. H. Jakobs, T. J. Law, "The Growth and Structure of Gold and Silver Deposits Formed by Evaporation Inside an Electron Microscope", Philos. Mag. 10 (1964) 127

[Pen69] W.B. Pennebaker, "Hillock Growth and Stress Relief in Sputtered Au Films", J. Appl. Phys. 40 (1969) 394

[Pen01] H. Y. Peng, N. Wang, W. S. Shi, Y. F. Zhang, C. S. Lee, S. T. Lee, "Bulkquantity $\mathrm{Si}$ nanosphere chains prepared from semi-infinite length $\mathrm{Si}$ nanowires", J. Appl. Phys. 89 (2001) 727

[Pet05] J. Petersen, S. G. Mayr, "Smoothening of internal phase boundaries by ion bombardment", J. Appl. Phys. 98 (2005) 033536

[Pet08] J. Petersen, S. G. Mayr, "Dewetting of Ni and NiAg solid thin films and formation of nanowires on ripple patterned substrates", J. Appl. Phys. 103 (2008) 023520

[Pie07] O. Pierre-Louis, A. Chame, Y. Saito, "Dewetting of a Solid Monolayer", Phys. Rev. Lett. 99 (2007) 136101

[Pre72] A. E. B. Presland, G. L. Price, D. L. Trimm, "Kinetics of Hillock and Island Formation During Annealing of Thin Silver Films", Prog. Surf. Sci. 3 (1972) 63

[Pre96] W. Press, M. Tolan, "Spekuläre und nicht-spekuläre Reflexion von Röntgen- und Neutronenstrahlen", 27. Ferienkurs, Streumethoden zur Untersuchung kondensierter Materie, (Forschungszentrum Jülich 1996)

[Ram98] Kathryn A. Ramirez-Aguilar and Kathy L. Rowlen, "Tip Characterization from AFM Images of Nanometric Spherical Particles", Langmuir 14 (1998) 2562

[Ran07] S. J. Randolph, J. D. Fowlkes, A. V. Melechko, K. L. Klein, H. M. Meyer III, M. L. Simpson, P. D. Rack, "Controlling thin film structure for the dewetting of catalyst nanoparticle arrays for subsequent carbon nanofiber growth", Nanotechnology 18 (2007) 465304 
[Ray78] L. Rayleigh, "On the Instability of Jets", Proc. London Math. Soc. 10 (1878) 4

[Red91] C. Redon, F. Brochard-Wyart, F. Rondelez, "Dynamics of dewetting", Phys. Rev. Lett. 66 (1991) 715

[Rei92] G. Reiter, "Dewetting of Thin Polymer Films", Phys. Rev. Lett. 68 (1992) 75

[Rei96] B. M. Reinker, "STM-Untersuchungen an amorphen ZrCo- und ZrAlCuAufdampfschichten", Dissertation, Universität Augsburg (1996)

[Rev78] P. Revesz, M. Wittmer, J. Roth, J. W. Meyer, "Epitaxial regrowth of Arimplanted amorphous silicon", J. Appl. Phys. 49 (1978) 5199

[Rol85] A. Rolland, B. Aufray, "Superficial composition in binary solid solutions A(B): Drastic effect of pure element surface tensions", Surf. Sci. 162 (1985) 530

[Ros01] C. A. Ross, "Patterned Magnetic Recording Media", Annu. Rev. Mater. Res. 31 (2001) 203

[Ros02] S. M. Rossnagel, T. S. Kuan, "Time development of microstructure and resistivity for very thin Cu films", J. Vac. Sci. Technol. A 20 (2002) 1911

[Rus98] S. Rusponi, G. Constantini, C. Boragno, U. Valbusa, "Ripple Wave Vector Rotation in Anisotropic Crystal Sputtering", Phys. Rev. Lett. 81 (1998) 2735; S. Rusponi, G. Constantini, C. Boragno, U. Valbusa, "Scaling Laws of the Ripple Morphology on Cu(110)", Phys. Rev. Lett. 81 (1998) 4184

[San99] P. Sandström, E. B. Svedberg, M. P. Johansson, J. Birch, J.-E. Sundgren, "Structure evolution in $\mathrm{Ag} / \mathrm{Ni}$ multilayers grown by ultra high vacuum DC magnetron sputtering", Thin Solid Films 353 (1999) 166

[Sas04] B. Sass, "Magnetische Grenzflächeneffekte in Doppellagen aus $\mathrm{V}_{2} \mathrm{O}_{3}$ und den ferromagnetisch geordneten Übergangsmetallen", Dissertation, Universität Göttingen (2004)

[Sax05] R. Saxena, M. J. Frederick, G. Ramanath, W. N. Gill, J. L. Plawsky, "Kinetics of voiding and agglomeration of copper nanolayers on silicon", Phys. Rev. B 72 (2005) 115425

[Sch00] L. Schmirgeld-Mignot, P. J. A. Molinàs-Mata, S. Poissonnet, G. Martin, "Reactive solid-state dewetting", Phil. Mag. Lett. 80 (2000) 33

[See01] R. Seemann, S. Herminghaus, K. Jacobs, "Shape of a Liquid Front upon Dewetting", Phys. Rev. Lett. 87 (2001) 196101

[See01a] R. Seemann, S. Herminghaus, K. Jacobs, "Gaining control of pattern formation of dewetting liquid films", J. Condens. Matter 13 (2001) 4925 
[See05] R. Seemann, S. Herminghaus, C. Neto, S. Schlagowski, D. Podzimek, R. Konrad, H. Mantz, K. Jacobs, "Dynamics and structure formation in thin polymer melt films", J. Phys.: Condens. Matter 17 (2005) S267

[Sei56] F. Seitz, J. Koehler, "displacement of atoms during irradiation", in "Solid State Physics", Vol. 2, edited by F. Seitz and D. Turnbull, (Academic Press, New York, 1956) 305

[Sha80] S. K. Sharma, J. Spitz, "Hillock Formation, Hole Growth and Agglomeration in Thin Silver Films", Thin Solid Films 65 (1980) 339

[Shi72] D. A. Shirley, "High-Resolution X-Ray Photoemission Spectrum of the Valence Bands of Gold", Phys. Rev. B 5 (1972) 4709

[Sie01] K. Sieradzki, K. Bailey, T. L. Alford, "Agglomeration and percolation conductivity", Appl. Phys. Lett. 79 (2001) 3401

[Sig69] P. Sigmund, "Theory of Sputtering. I. Sputtering Yield of Amorphous and Polycrystalline Targets", Phys. Rev. 184 (1969) 383

[Sig73] P. Sigmund, "A mechanism of surface micro-roughening by ion bombardment", J. of Mater. Sci. 8 (1973) 1545

[Sig80] P. Sigmund, A. Gras-Marti, "Distortion of Depth Profiles During Sputtering", Nucl. Instrum. Methods 168 (1980) 389

[Sig81] P. Sigmund, A. Gras-Marti, "Theoretical Aspects of Atomic Mixing by Ion Beams", Nucl. Instrum. Methods 182/183 (1981) 25

[Smi95] D. L. Smith, "Thin Film Deposition, Principles and Practice", (New York, McGraw-Hill Book, Inc., 1995)

[Son52] E. H. Sondheimer, "The mean free path of electrons in metals", Advan. Phys. 1 (1952) 1

[Spe93] B. J. Spencer, P. W. Voorhees, S. H. Davis, "Morphological instability in epitaxially strained dislocation-free solids: Linear stability theory", J. Appl. Phys. 73 (1993) 4955

[Spi89] E. Spiller, "Smoothing of multilayer x-ray mirrors by ion polishing", Appl. Phys. Lett. 54 (1989) 2293

[Sro86] D. J. Srolovitz, S. A. Safran, "Capillary Instabilities in thin films. I. Energetics", J. Appl. Phys. 60 (1986) 247

[Sro89] D. J. Srolovitz, " On the stability of surfaces of stressed solids", Acta Metall. 37 (1989) 621

[Sro95] D. J. Srolovitz, M. G. Goldiner, "The Thermodynamics and Kinetics of Film Agglomeration", JOM 47 (1995) 31 
[Str99] G. J. Strijkers, J. H. J. Dalderop, M. A. A. Broeksteeg, H. J. M. Swagten, W. J. M. de Jonge, "Structure and magnetization of arrays of electrodeposited Co wires in anodic alumina", J. Appl. Phys. 86 (1999) 5141

[Sug97] A. Sugawara, M. R. Scheinfein, "Room-temperature dipole ferromagnetism in linear-self-assembling mesoscopic Fe particle arrays", Phys. Rev. B 56 (1997) 8499

[Sut06] P. Sutter, W. Ernst, Y. S. Choi, E. Sutter, "Mechanisms of thermally induced dewetting of ultrathin silicon-on-insulator", Appl. Phys. Lett. 88 (2006) 141924

[Tak07] K. Takahiro, K. Zhang, F. Rotter, D. Schwen, C. Ronning, H. Hofsäss, J. Krauser, "Morphological change of carbon surfaces by sputter erosion", Nucl. Instrum. Meth. B 256 (2007) 378

[Tho00] C. V. Thompson, "Structure Evolution During Processing of Polycrystalline Films", Annu. Rev. Mater. Sci. 30 (2000) 159

[Tho77] J. A. Thornton, "High Rate Thick Film Growth", Ann. Rev. Mater. Sci. 7 (1977) 239

[Tob98] E. D. Tober, R. F. C. Farrow, R. F. Marks, G. Witte, K. Kalki, D. D. Chambliss, "Self-Assembled Lateral Multilayers from Thin Film Alloys of Immiscible Metals", Phys. Rev. Lett. 81 (1998) 1897

[Tou85] S. Tougaard, B. Jörgensen, "Absolute Background Determination in XPS", Surf. Interface Anal. 7 (1985) 17

[Tro90] G. Tréglia, B. Legrand, P. Maugain, "Surface Segregation in CuNi and AgNi Alloys Formulated as an Area-Preserving Map", Surf. Sci. 225 (1990) 319

[Tun80] R. T. Tung, W. R. Graham, "Single Atom Self-Diffusion on Nickel Surfaces", Surf. Sci. 97 (1980) 73

[Tus03] C. Tusche, "Magnetische Kopplung und Grenzflächenanisotropie von Eisen/Vanadiumsesquioxid- und Kobalt/Vanadiumsesquioxid-Doppellagen", Diplomarbeit, Universität Göttingen (2003)

[Ued76] Y. Ueda, M. Sato, "Magnetic anisotropy in sinlge-crystal nickel films electrodeposited on a (110) copper-nickel crystal surface", J. Appl. Phys. 47 (1976) 3380

[Uhr85] M. Uhrmacher, K. Pampus, F. J. Bergmeister, D. Purschke, K. P. Lieb, "Energy calibration of the $500 \mathrm{kV}$ heavy ion implanter IONAS", Nucl. Instrum. Meth. B 9 (1985) 234 
[Umb01] C. C. Umbach, R. L. Headrick, K.-C. Chang, "Spontaneous Nanoscale Corrugation of Ion-Eroded $\mathrm{SiO}_{2}$ : The Role of Ion-Irradiation-Enhanced Viscous Flow", Phys. Rev. Lett. 87 (2001) 246104

[Umb06] C. C. Umbach, Department of Materials Science and Engineering, Cornell University, persönliche Kommunikation, SFB 602 OTF-Workshop, Göttingen (2006)

[Vaj96] J. J. Vajo, R. E. Doty, E.-H. Cirlin, "Influence of $\mathrm{O}_{2}^{+}$energy, flux, and fluence on the formation and growth of sputtering-induced ripple topography on silicon", J. Vac. Sci. Technol. A 14 (1996) 2709

[Vau07] S. Vauth, S. G. Mayr, "Relevance of surface viscous flow, surface diffusion, and ballistic effects in keV ion smoothing of amorphous surfaces", Phys. Rev. B 75 (2007) 224107

[Whi02] G. M. Whitesides, B. Grzybowski, "Self-Assembly at All Scales", Science 295 (2002) 2418

[Wie94] R. Wiesendanger, "Scanning Probe Microscopy and Spectroscopy: Methods and Applications", (Cambridge University Press, Cambridge, GB, 1994)

[Won00] H. Wong, P. W. Voorhees, M. J. Miksis, S. H. Davis, "Periodic Mass Shedding of a Retracting Solid Film Step", Acta Mater. 48 (2000) 1719

[Wu04] W. Wu, S. H. Brongersma, M. van Hove, K. Maex, "Influence of surface and grain-boundary scattering on the resistivity of copper in reduced dimensions", Appl. Phys. Lett. 84 (2004) 2838

[Xia91] Z. G. Xiao, G. A. Canovai, C. M. Osburn, "Agglomeration of cobalt silicide films" Mat. Res. Soc. Symp. Proc. 202 (1991) 101

[You05] T. Young, "An Essay on the Cohesion of Fluids", Philos. Trans. R. Soc. London 95 (1805) 65

[Zei04] Carl Zeiss NTS GmbH, "Handbuch für die Rasterelektronenmikroskope SUPRA(VP) und ULTRA", (2004)

[Zha00] M. Zhang, M. Y. Efremov, F. Schiettekatte, E. A. Olson, A. T. Kwan, S. L. Lai, T. Wisleder, J. E. Greene, L. H. Allen, "Size-dependent melting point depression of nanostructures: Nanocalorimetric measurements", Phys. Rev. B 62 (2000) 10548

[Zib05] B. Ziberi, F. Frost, Th. Höche, B. Rauschenbach, "Ripple pattern formation on silicon surfaces by low-energy ion-beam erosion: Experiment and theory", Phys. Rev. B 72 (2005) 235310

[Zie85] Z. F. Ziegler, J. P. Biersack, U. Littmark, "Stopping and Ranges of Ions in Solids", Vol. 1, (Pergamon Press, New York, 1985), (verwendeter Programm-Code: SRIM2003) 
[Zim01] C. G. Zimmermann, K. Nordlund, M. Yeadon, J. M. Gibson, R. S. Averback, U. Herr, K. Samwer, "Burrowing of nanoparticles on clean metal substrates: Surface smoothing on a nanoscale", Phys. Rev. B 64 (2001) 085419 



\section{Danksagung}

Ich möchte mich an dieser Stelle bei all denen bedanken, die zum Gelingen dieser Arbeit beigetragen haben.

Prof. Dr. S. G. Mayr danke ich dafür, dass er mir das Anfertigen dieser Dissertation innerhalb der anfangs noch sehr jungen und im Aufbau befindlichen Arbeitsgruppe ermöglicht hat. Seine Unterstützung und das Interesse am Fortgang dieser Arbeit sowie der gewährte Gestaltungsfreiraum haben mich stets motiviert.

Prof. Dr. R. Kirchheim möchte ich dafür danken, dass er das Korreferat dieser Arbeit übernommen hat.

PD Michael Seibt, Dr. Karsten Thiel und Volker Radisch danke ich für die mehr als tatkräftige Unterstützung bei den Messungen am TEM und der Präparation mittels FIB. Ebenso sei Dr. Marija Djordjevic Kaufmann für die Zusammenarbeit während der gemeinsamen Stunden an der MOKE-Apparatur und insbesondere Dr. K. Zhang für die Durchführung von Bestrahlungen am IONAS gedankt.

Ich bedanke mich für die finanzielle Förderung durch die deutsche Forschungsgemeinschaft im Rahmen des Sonderforschungsbereichs 602.

Carsten Mahn und Uta Bete möchte ich danken für die Hilfe speziell bezüglich manch technischer Details und nützliche Tipps für die Umbauarbeiten an und in den diversen UHV-Anlagen, sowie Katrin Gehrke für das Abwechseln beim Justieren und Basteln an der Röntgenanlage (im Rahmen der Sicherheitsbestimmungen!). Außerdem verdient die feinmechanische Werkstatt meinen Dank für die präzise Anfertigung benötigter Bauteile, sei es Metall, Keramik oder auch nur ein Stück Plastik.

Unserer Arbeitsgruppe sowie allen Mitarbeitern des I. Physikalischen Instituts danke ich für das hervorragende Arbeitsklima und die freundliche Atmosphäre insbesondere auch während der außerdienstlichen Aktivitäten in Form von Grill- und Fußballabenden und der alljährlichen Seminarfahrten. Es fanden sich immer hilfsbereite Ansprechpartner, was die Grundlage einer guten Zusammenarbeit darstellt.

Speziellen Dank gilt dabei unserer Volleyball-Ikone Christian Vree, der stets versuchte, die Ehre des Instituts beim Sommerfestturnier zu retten. Er übernahm die Aufgabe des Lektors dieser Arbeit und konnte den einen oder anderen Fehler noch ausmerzen.

Dr. Markus (Harry) Eßeling, meinem Bürokollegen und ewigem Mitstreiter auf dem Weg durch das Studium, danke ich für eine äußerst unterhaltsame Zeit bei einer Vielzahl 
von wissenschaftlichen und unwissenschaftlichen Diskussionen über Gott und die Welt und eine gute Zusammenarbeit. Langeweile ist in unserem Büro schon allein auf Grund des Besucheraufkommens beispielsweise durch unsere italienischen und russischen "Sprachpatienten" Dr. Alberto Bracchi und Dr. Dimitrij Bogdanov sicherlich niemals aufgekommen.

Iris Kock bin ich dankbar für gemeinsame Arbeiten am Heizeinsatz und besonders für die moralische Unterstützung durch ihre liebevolle und heitere Art, mit der sie beharrlich gute Laune verbreitete.

Nicht zuletzt danke ich meiner Familie für die stete Unterstützung in allen Lebenslagen, die mir das Studium überhaupt erst ermöglicht hat. 


\section{Lebenslauf}

\section{Persönliche DAten:}

Name:

Geburtsdatum:

Geburtsort:

Staatsangehörigkeit:

\section{HochSCHULAUSBILDUNG}

$10 / 1997$

$12 / 2001-05 / 2003$

$07 / 2003$

$12 / 2003$
Jan Petersen

25.12.1975

Eckernförde

deutsch

Aufnahme des Physikstudiums an der Georg-August-Universität Göttingen

Diplomarbeit bei Prof. Dr. K. Samwer am I. Physikalischen Institut mit dem Thema

"Benetzungsverhalten des metallischen, glasbildenden Systems $\mathrm{Pd}_{43} \mathrm{Ni}_{10} \mathrm{Cu}_{27} \mathrm{P}_{20}$ auf unterschiedlichen Substraten"

Diplom in Physik

Beginn der Promotion bei Prof. Dr. S. G. Mayr am

I. Physikalischen Institut 